Florida International University

FIU Digital Commons

FIU Electronic Theses and Dissertations

University Graduate School

$11-8-2013$

\title{
The Effects of Age, Ethnicity, Sexual Dysfunction, Urinary Incontinence, Masculinity, and Relationship with the Partner on the Quality of Life of Men with Prostate Cancer
}

Suha Ballout

Florida International University, sball004@fiu.edu

DOI: $10.25148 /$ etd.FI13120415

Follow this and additional works at: https:// digitalcommons.fiu.edu/etd

Part of the Family Practice Nursing Commons, Gender and Sexuality Commons, Geriatric Nursing Commons, Male Urogenital Diseases Commons, and the Race and Ethnicity Commons

\section{Recommended Citation}

Ballout, Suha, "The Effects of Age, Ethnicity, Sexual Dysfunction, Urinary Incontinence, Masculinity, and Relationship with the Partner on the Quality of Life of Men with Prostate Cancer" (2013). FIU Electronic Theses and Dissertations. 978.

https://digitalcommons.fiu.edu/etd/978

This work is brought to you for free and open access by the University Graduate School at FIU Digital Commons. It has been accepted for inclusion in FIU Electronic Theses and Dissertations by an authorized administrator of FIU Digital Commons. For more information, please contact dcc@fiu.edu. 


\section{FLORIDA INTERNATIONAL UNIVERSITY \\ Miami, Florida}

THE EFFECTS OF AGE, ETHNICITY, SEXUAL DYSFUNCTION, URINARY

INCONTINENCE, MASCULINITY, AND RELATIONSHIP WITH THE PARTNER

ON THE QUALITY OF LIFE OF MEN WITH PROSTATE CANCER

A dissertation submitted in partial fulfillment of the

requirements for the degree of

DOCTOR OF PHILOSOPHY

in

NURSING

by

Suha Ballout

2013 
To: Dean Ora Lea Strickland

College of Nursing and Health Sciences

This dissertation, written by Suha Ballout, and entitled The Effects of Age, Ethnicity, Sexual Dysfunction, Urinary Incontinence, Masculinity, and Relationship with the Partner on the Quality of Life of Men with Prostate Cancer, having been approved in respect to style and intellectual content, is referred to you for judgment.

We have read this dissertation and recommend that it be approved.

$\begin{array}{r}\hline \text { Ellen Brown } \\ \hline \text { Carol A. ("Pat") Patsdaughter, Co-Major Professor } \\ \hline \text { Anahid Kulwicki, Co-Major Professor }\end{array}$

Date of Defense: November 8, 2013

The dissertation of Suha Ballout is approved.

$\begin{array}{r}\begin{array}{r}\text { Dean Ora Lea Strickland } \\ \text { College of Nursing and Health Sciences }\end{array} \\ \hline \begin{array}{r}\text { Dean Lakshmi N. Reddi } \\ \text { University Graduate School }\end{array}\end{array}$

Florida International University, 2013 
(C) Copyright 2013 by Suha Ballout

All rights reserved. 


\section{ACKNOWLEDGMENTS}

The journey of my doctoral studies and dissertation was successful attributable to the patience, support, and guidance of my committee, professors, friends, and family. Moving from Beirut to Miami was a life changing experience and the beginning of a dream comes true. The dissertation process was full of challenges and difficulties but the support, guidance, and encouragement of my committee made it possible. Dr. Anahid Kulwicki, was my chair, boss, mentor, friend, and so much more. Working with Dr. Kulwicki, I gained a wealth of knowledge about the qualities of a successful nursing leader and scholar. She believed in my abilities and capabilities and pushed me to deliver my best. She guided me to articulate my program of research and "what's its name", my dissertation study. At the end, despite the distance and not studying Arab men, Dr. Kulwicki's feedback kept me grounded in making my study a foundation for a future program of research. Dr. Carol "Pat" Patsdaughter was my professor, mentor, friend, cheerleader, and role model. Dr. Patsdaughter provided me with support throughout the program and molded me in to being a junior scholar. Dr. Pats continuously and repeatedly reinforced that "a good dissertation is a done dissertation" until I was actually done! Dr. Patsdaughter's care and support encouraged me to overcome all the data collection challenges and obstacles smoothly. Since our first encounter, she has motivated me and held me to the highest standards. She also encouraged me to develop my service and be part of the consortium of south Florida research conference planning committee. She supported my decisions and management of the study through long conversations where I had to justify every step and "add a reference" to every thought. As a result, I developed an in-depth relationship with my study, data, and aspirations for 
future research. Along the way, I discovered great interest in editing and I look forward to enhancing this skill further under the mentorship of Dr. Patsdaughter. Dr. Pats from the royal wedding to the dissertation defense, you have been my family in Miami.

I also want to thank Dr. Ellen Brown for the valuable advice and discussions. My conversations with Dr. Brown suggested different avenues where I can take my future work. Thank you! Dr. Abraham Lavendar contributed to my work with important and interesting discussions about masculinity and sexuality of men from various cultures. $\mathrm{He}$ joined Dr. Kulwicki in convincing me of the importance of conducting a similar study with Arab men. So, I promise that it will be on my "to do list" to examine my concepts with a sample of Arab men.

I would also like to thank my nursing professors and mentors. Dr. Dorothy Brooten and Dr. JoAnne Youngblut were very influential and nurturing during my courses and thereafter. They provided me a wealth of knowledge about the research process, grant applications, and methods of becoming a successful and funded researcher. I will always remember to present my findings as if talking to the "lady at the bus stop." Also, I will make sure that my grant applications will describe "So what? Why is it so important to fund you to conduct this study.... and where does this fall in your program of research?" in details to have my application scored and funded. Dr. Luz Porter was also very nurturing, supportive, and encouraging. Despite not taking courses with her, Dr. Sande Garcia Jones provided me with ample support and advice that helped shape my research design. Dr. Eric Fenkl, I looked forward to our conversations and exchange of ideas and I hope we get to work together. Dr. Janyce Dyer also supported my journey and encouraged me. In fact, all the faculty and staff at the College of Nursing and Health 
Sciences were very supportive. Dean Divina Grossman welcomed me into the program and was confident that I will have a successful experience and consequently would lead a flourishing professional career. Dean Ora Strickland set the bar so high and promoted me to continuously seek a research intensive career path. She is a prominent researcher, a nurse leader, and my role model.

I also want to thank my colleagues and partners in the journey at FIU. Frank Guido-Sanz, from my first day at FIU and in Miami, you were my best friend, studying and conference partner, guide to Miami, and sometimes my GPS. We worked well together through courses and maintained our working bond through candidacy and dissertation days in addition to other days. We keep each other grounded and going. Interestingly, you were able to see my strengths with statistics and weaknesses in following directions and reversing my car. We shared many laughs, tears, and long chats in addition to many many meals and good times. I will be looking forward to seeing you Dr. Frank soon! Raquel Vera, we were twins during the dissertation process. You too were my angel. Thank you for your support and for being my friend. Carmen Caicedo, it is a pleasure to be your friend. Your continuous positive outlook on everything reminded me that there are better days yet to come! You are an inspiration and a true friend. Paula Glass, we shared the journey and I could not have started my study without you. You were my supporter and reference when I started teaching. I would also like to thank Dawn Hawthorne, Rosi Roche, Ivette Hidalgo, Cora Yoose, and all my colleagues. Moreover, I would like to mention the students who worked with me on my study especially Aliki Vasiliadi, Niurka Parrado, and Jessica Sanabria. I would also like to 
thank Dr. Sharon Simon and Dr. Maria Olenik for supporting me in balancing my teaching responsibilities and student role.

My journey could not have been fun, smooth, stressful, and successful without the ultimate support, patience, and care of my lovely friends. I hope I have enough words to thank you enough. Hiba Bou Akar, time zones, busy schedules, and crazy life events separated us, but you still managed to provide me with the most valuable support on so many levels. We laughed, stressed out, cried, talked for hours, and found time to share professional advice frequently. You elevated me during stressful times and encouraged me to transform my lifestyle to include healthy cooking and exercise while maintaining a busy work schedule and all the other social stuff I engaged myself in. Through discussions of war and "war yet to come" to socially constructed gender norms, prostate cancer, and quality of life, we managed to compare notes between the processes of conducting ethnographies versus quantitative research designs. We learned from each other and built our passion to what we do. Do you think that we could join our areas of research? I wonder what that would be like? Claude Chidiac, "Coco", thank you for being you! You are a very close friend, a younger brother, a confidant. Being in Dublin did not stop you from being very supportive of every step of my dissertation, although sometimes you had no idea where I was going with my concerns. Without knowing, you inspired me in so many ways to keep on going despite all the challenges and obstacles. No matter how crazy I got, you were always there to ground me and bring me back to reality, "Nmnmnmnm." The "boota” or "gang": Zeina Kassem, Janane Hanna, Faten Badran, Rebecca El Asmar, Hala Richa, and Claude Chidiac, you were with me every day for so many years especially when I moved to Miami. Waking up and looking at 
your souvenir poster with all our pictures, I was inspired and focused to fulfill the promise and "one more time..." Claude, Zeina, and Janane, your visit meant a lot to me and preserved my sanity. Mazen Ghaziri and Sara Abboud, you made distance and time undetectable through maintaining our crazy communication, just like the old days. Our chats retained a sense of normalcy. Samira Hemadeh Kerbage, our days and nights never met; however, you still found ways to be closer than my next door neighbors. We started our nursing journey together and dreamt of being nurse leaders. Despite all odds, we are both on the journey. Looking back, we share many memories from nursing school days, to working at AUBMC, traveling around, and many more. You always were by my side during happy moments, but mostly you were there when I was fighting for my life. Samira, we are more than friends, we share life changing experiences and distance would not come between us. Rana Abu Dargham, I miss you! Rudy, you helped me during my beginnings and there were so many things that I could not have done without you. Ali Davis, you provided me with meaningful support and encouragement. You repeatedly told me that I can do it all and thank you for keeping me on the go. Rasha Hamzeh, thank you for checking on me every now and then and I looked forward to your "Ballouta, how's life?" Mirna Harb Abdo and Ibrahim Abdo, I look forward to your annual March visit. From Verdun to Miami, we managed to meet and make others jealous. Please please please, let's maintain the tradition and keep meeting all over the world. Most importantly, Gregory Scarbriel, thank you for everything! Again, I assure you that you were not part of my study but I sure did enjoy discussing my findings with you. You encouraged and supported me in every step of my study despite being "confused" about what I was talking about. You managed to look interested in what I was doing and 
accompanied me through every chapter of my dissertation. You gave me deadlines for chapters 4 and 5 and made sure I met them. You listened and lived through my panic moments and my happy accomplishments. In the end, you were the first person to celebrate my success and be happy that I was done! Thank you for everything and looking forward to sharing many Tings together. I also want to thank your family for their support and making me feel that I have a home away from home.

Finally, I would like to thank my dad, Latif Ballout, and my brother, Ramzi Ballout. I could not be here without your continuous and ultimate support. Despite the distance, you managed to provide me with love and encouragement through every step and milestone. Daddy, you are my role model and my hero. I am who I am today because of your ongoing support. I have always looked up to you. Growing up, I wanted to make you proud and be successful like my daddy. Despite my stubbornness, you always manage to help me understand what is most important to keep going. No matter what I decided to do, you were there to be proud of me and applaud my success and also to lift me up when I fall. Constantly, you are one step ahead of me and asking me what's next. I promise, I will always make you proud! Ramzi, distance could not come between our long conversations about every possible topic. We exhausted every existing program and application to communicate with each other, see each other, and share screens from Miami to Dubai and all over the world where you travelled. You were there around the clock as my best friend and my lovely brother. Habibi, I am proud to be your sister and I will always remember that there are areas where you can achieve better! No matter where we are and what we are doing; we will always be each other's back bone. I don't want to forget my aunts and cousins: Bassoum, Rashed, Nadim, Sara, Hoda, Souheil, 
Elvis, Scarlet, and Marvin. Your supportive messages and calls made me feel loved and brought the warmth of family from Lebanon to Miami. To my mom, thank you for checking on me every now and then and being proud of my accomplishments. In summary, thank you all for being the most supportive parents! 


\begin{abstract}
OF THE DISSERTATION
THE EFFECTS OF AGE, ETHNICITY, SEXUAL DYSFUNCTION, URINARY

INCONTINENCE, MASCULINITY, AND RELATIONSHIP WITH THE PARTNER

ON THE QUALITY OF LIFE OF MEN WITH PROSTATE CANCER
\end{abstract}

by

Suha Ballout

Florida International University, 2013

Miami, Florida

Professor Anahid Kulwicki, Major Professor

Professor Carol A. ("Pat”) Patsdaughter, Co-Major Professor

Prostate cancer, the leading cause of cancer in men, has positive survival rates and constitutes a challenge to men with its side effects. Studies have addressed the bivaritate relationships between prostate cancer treatment side effects masculinity, partner relationship, and quality of life (QOL). However, few studies have highlighted the relationships among prostate cancer treatment side effects (i.e., sexual dysfunction, urinary incontinence), masculinity, and relationship with the partner together on QOL in men. Most studies were conducted with predominately Caucasian sample of men. Miami is a unique multiethnic setting that hosts Cuban, Columbian, Venezuelan, Haitian, other Latin American and Caribbean communities that were not represented in previous literature. The purpose of this study was to examine relative contributions of age, ethnicity, sexual dysfunction, urinary incontinence, masculinity, and perception of the relationship with the partner on the quality of life in men diagnosed with prostate cancer. Data were collected using self-administered questionnaires measuring demographic 
variables, sexual and urinary functioning (UCLA PCI), masculinity (CMNI), partner relationship (DAS), and QOL (SF-36). A total of 117 partnered heterosexual men diagnosed with prostate cancer were recruited from four urology clinics in Miami, Florida. Men were $67.47(S D=8.42)$ years old and identified themselves to be of Hispanic origin $(54.3 \%, n=63)$. Findings demonstrated that there was a significant moderate negative relationship between urinary and sexual functioning of men. There was a significant strong negative association between men's perceived relationship with partner and masculinity. There was a weak negative relationship between the partner relationship and QOL. Hierarchal multiple regression showed that the partner relationship $(\beta=-.25, t(91)=-2.28, p=.03)$ significantly contributed overall to QOL. These findings highlight the importance of the relationship satisfaction in the QOL of men with prostate cancer. Nursing interventions to enhance QOL for these men should consider strengthening the relationship and involving the female partner as an active participant. 


\section{TABLE OF CONTENTS}

CHAPTER

PAGE

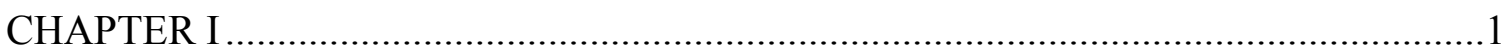

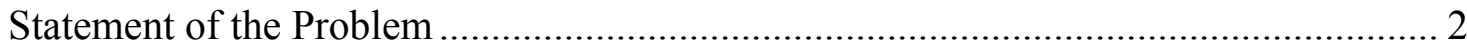

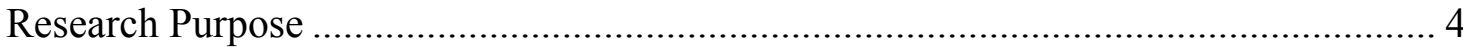

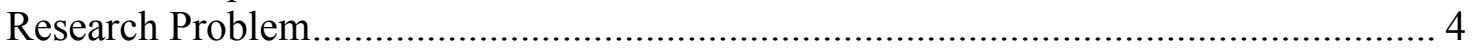

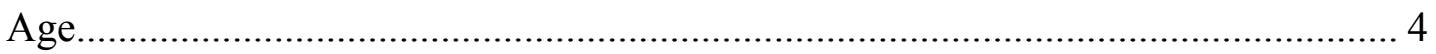

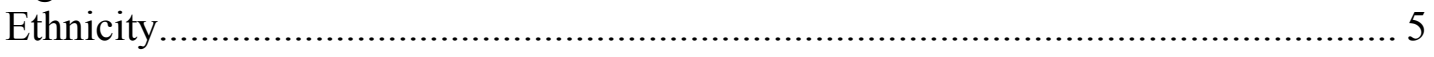

Prostate Cancer Treatment Side Effects ................................................................. 7

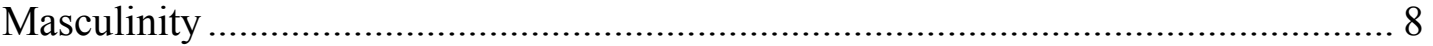

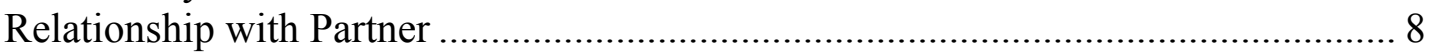

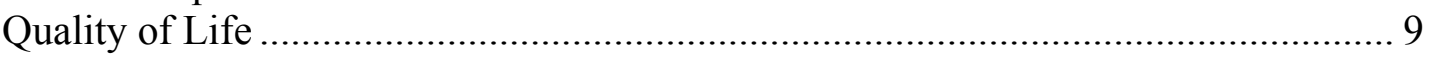

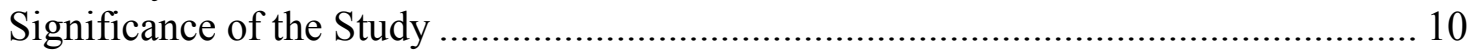

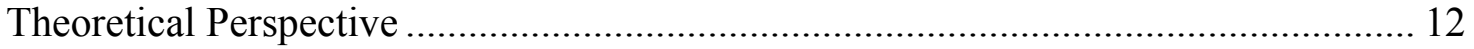

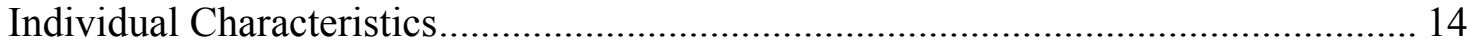

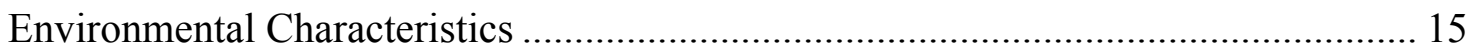

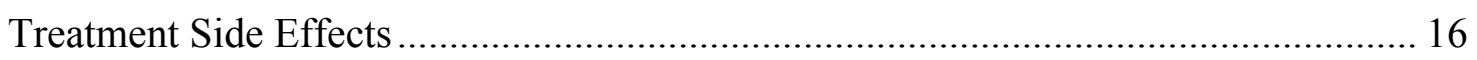

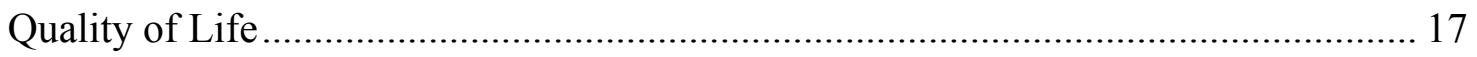

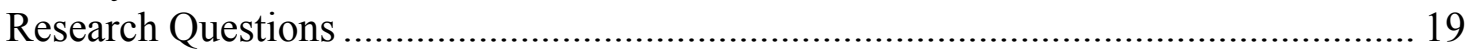

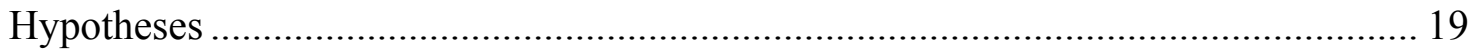

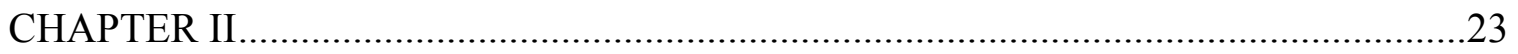

Epidemiology of Prostate Cancer........................................................................... 24

Prostate Cancer Treatment and Side Effects …………................................................ 27

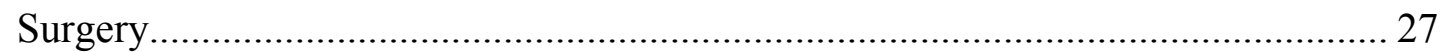

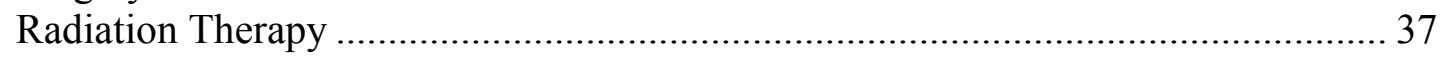

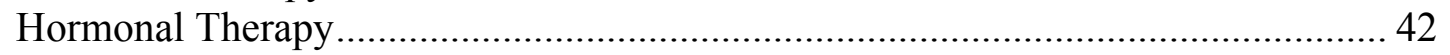

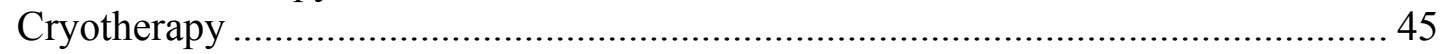

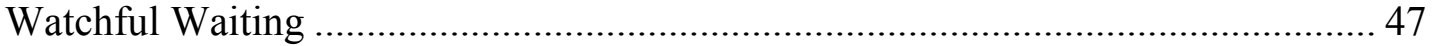

Comparison of Treatment Options ....................................................................... 52

Economic Perspective of Prostate Cancer ............................................................ 58

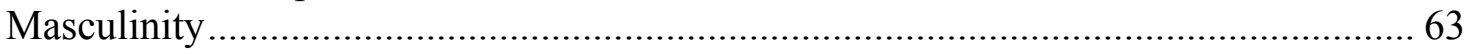

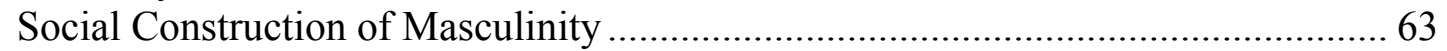

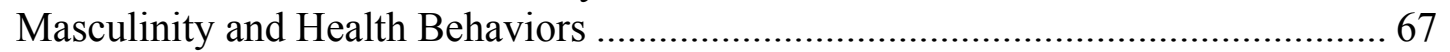

Masculinity and Prostate Cancer ........................................................................ 75

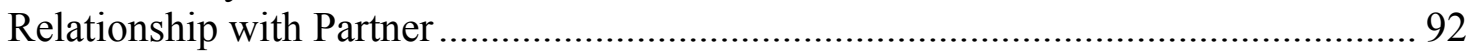

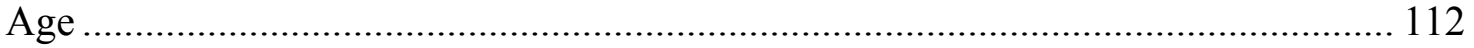

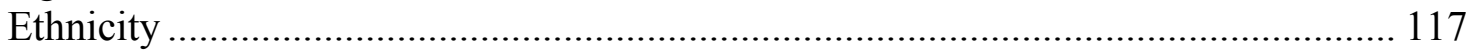

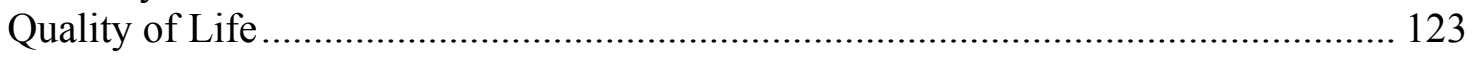

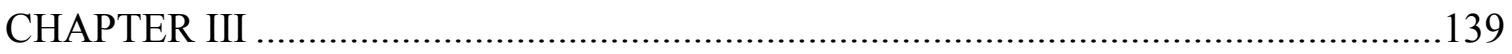

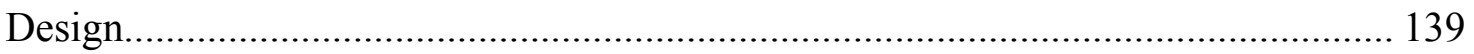

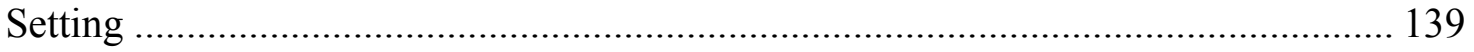




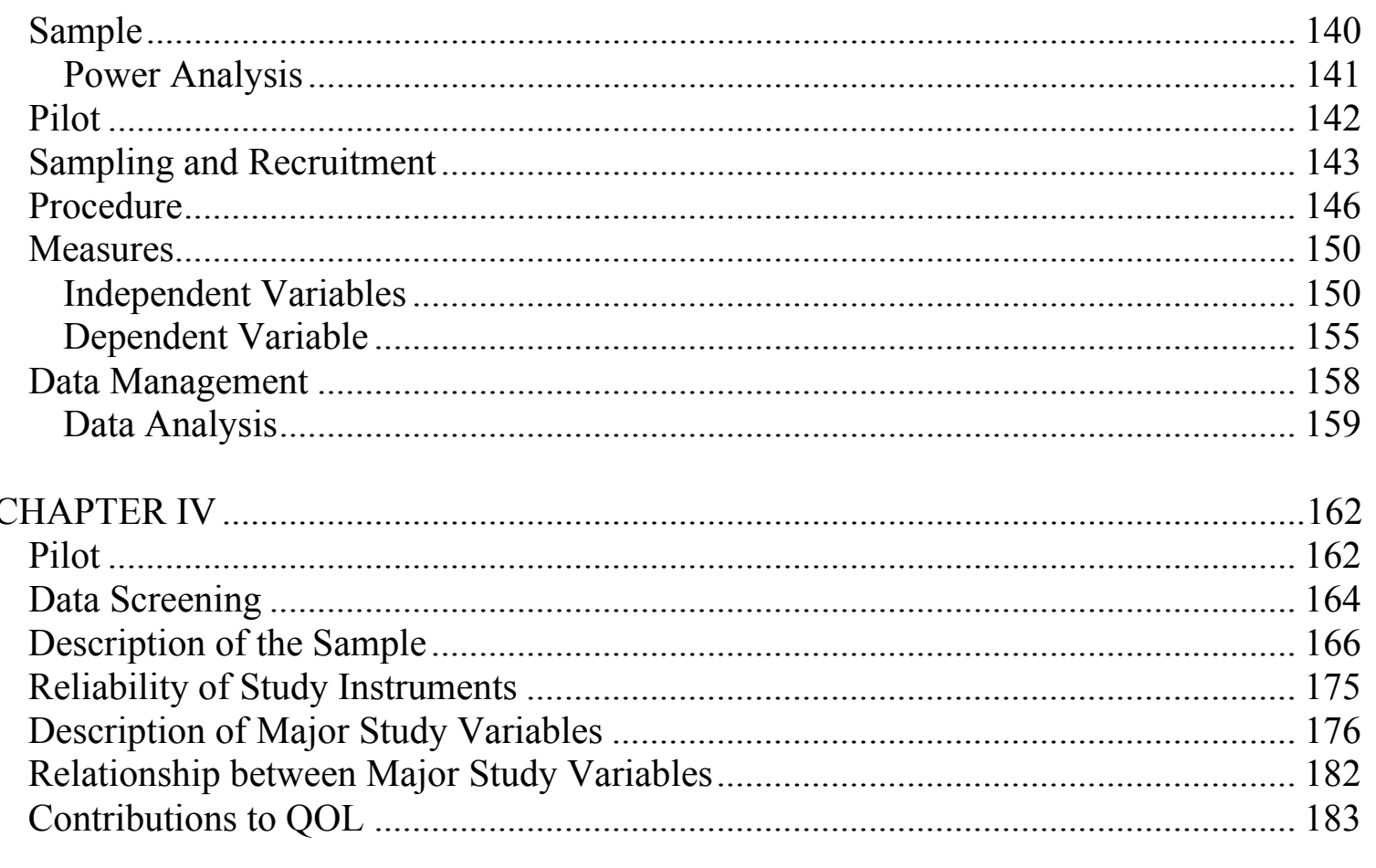

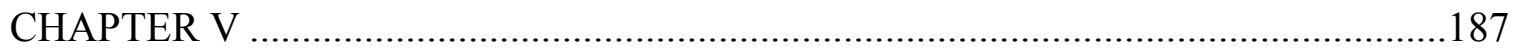

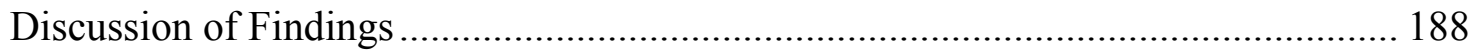

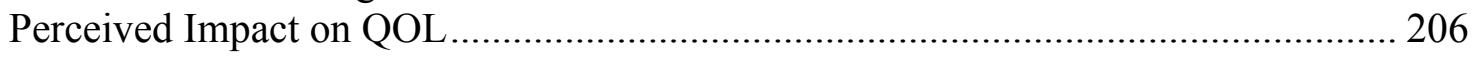

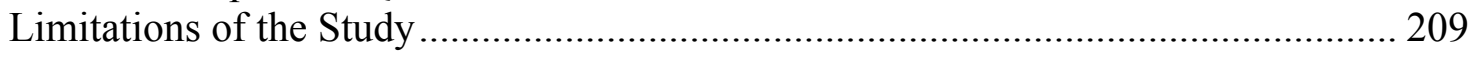

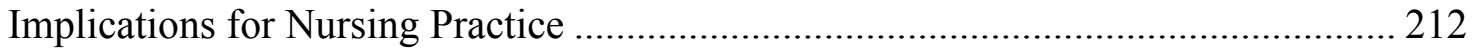

Implications for Future Research ............................................................... 214

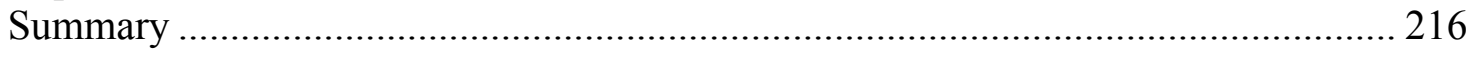

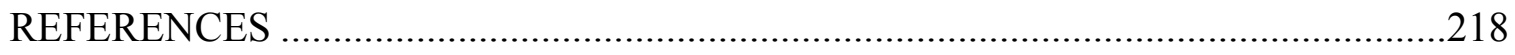

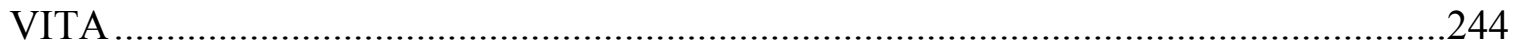




\section{LIST OF TABLES}

TABLE

PAGE

1. Major Study Variables............................................... 156

2. Demographic Characteristics of the Sample................................... 166

3. Disease Status..................................................... 170

4. Participation of Men in Treatment Decision.................................. 172

5. Sexual Issues related to Prostate Cancer.................................... 174

6. Description of Major Study Variables..................................... 175

7. Relationships Between Age, Ethnicity, Sexual Dysfunction, Urinary Incontinence, Masculinity, and Perception of the Relationship with the Partner, and Quality of Life. 182

8. Relative Contributions to QOL in Men with Prostate Cancer. 185 


\section{CHAPTER I}

\section{INTRODUCTION}

Cancer is a major public health concern that has steadily increased in incidence rates and prevalence of diagnosed cases. The American Cancer Society (ACS) has estimated the incidence rate of prostate cancer in 2013 to be 151.4 in 100,000 with an estimate of 238,590 new diagnosed cases (ACS, 2013). Over the past decade, there has been a significant increase in the prevalence of the annual cancer incidence (Jemal, Siegel, Xu, \& Ward, 2010; Siegel, Naishadham, \& Jemal, 2013). The increased prevalence is attributed to improved awareness and diagnostic technology (Jemal et al., 2006). The majority of cancer sites have reported a slight decrease in mortality rates in recent years which may be due to the advancement of diagnostic and treatment options (Jemal et al., 2010). With the advancement of technology, cancer treatment options are widely available. However, the majority of these treatments are accompanied by side effects that encompass physical (e.g., body image and physical changes, sexual dysfunction, anemia), psychological (e.g., anxiety, depression), social, economic, and other consequences.

With increased prostate cancer prevalence, more people are living with their disease, symptoms, and treatment side effects that are interrupting their lives and expected social roles and responsibilities. As a result, these men are suffering from physical conditions (i.e., the cancer, its physiological symptoms, and treatment side effects) and compromised social expectations (i.e., ability to meet their socially constructed roles at home, work, and among friends). This disruption is affecting cancer 
patients' physical functioning, relationships, social roles, quality of life (QOL) (Kaasa, Mastekaasa, \& Lund, 2003) and contributing to their mortality (Chida \& Steptoe, 2008).

\section{Statement of the Problem}

Prostate cancer accounts for 238,590 (28\%) of cancer incidence and is the leading cancer in men. It is estimated that one in six men will have prostate cancer in their lifetimes (National Cancer Institute [NCI], 2013; Siegel et al., 2013). The ACS reported that 241,740 new cases were diagnosed in 2012 in the United States, and the estimates for 2013 are about 238,590 new diagnosed cases of prostate cancer (ACS, 2013). In 2013 in Florida, the incidence of prostate cancer was 137.7 in 1000,000, and it was estimated that 17,330 new cases were diagnosed with prostate cancer (ACS, 2013; Siegel et al., 2013). The majority of prostate cancer was localized (80\%), followed by regional (12\%) and distal (6\%) (Altekruse et al., 2010). Epidemiologic trends have shown that more men are living longer with prostate cancer (Jemal et al., 2006; Jemal et al., 2010; Siegel et al., 2013). Unlike other cancers, the 5-year survival rate of $100 \%$ is a positive aspect of the disease (Jemal et al., 2010; Siegel et al., 2013). Being the leading cause of cancer in men, it is essential to examine the impact of prostate cancer and treatment on men's QOL, especially since they are living longer after the diagnosis of cancer.

Prostate cancer treatment options vary, and research has not yet provided evidence of differential outcomes of surgery, radiation, hormonal therapy, or watchful waiting (Hoznek, Menard, Salomon, \& Abboud, 2005; Wei et al., 2002). Nevertheless, there is a growing number of studies that have documented the side effects of these treatments including sexual dysfunction (30-80\%), urinary incontinence (30-74\%), and bowel incontinence (3-10\%) (Hoznek et al., 2005; Wei et al., 2002). Most studies have 
identified sexual dysfunction to be the most significant and bothersome side effect of prostate cancer treatment (Hoznek et al., 2005; Wei et al., 2002).

Research that has examined the impact of side effects of prostate cancer treatment on QOL in men has addressed the challenge to masculinity that may influence the relationship with their partner (Sanders, Pedro, Bantum, \& Galbraith, 2006). With masculinity being identified as a major concern, sexual dysfunction may create a dilemma for these men (Oliffe, 2005). This dilemma arises from the premise that sexuality and sexual functioning are imperative for a man's wellbeing. Studies have accounted for QOL, sexuality, and relationship with the partner as being the main outcome indicators of prostate cancer treatment (Badr \& Carmak Taylor, 2009; Giarelli, McCorckle, \& Monturo, 2003). The impact of prostate cancer treatment side effects on QOL (Howlet et al., 2010; Monga, Kerrigan, Thornby, Monga, \& Zimmermann, 2005), masculinity (Burns \& Mahalik, 2006; Fergus, Grey, \& Fich; 2002), and partner relationship (Galbraith, Pedro, Jaffe, \& Allen, 2008; Sanders et al., 2006) have been examined. However, research has not highlighted the relationships among prostate cancer treatment side effects (i.e., sexual dysfunction, urinary incontinence), masculinity, and relationship with the partner together on QOL in men. Also, the majority of studies have been conducted with predominantly Caucasian samples. As a result, the experiences of men from other ethnic populations have not been adequately represented in the literature, despite ethnicity being a major risk factor for the disease (Jemal, Siegel, $\mathrm{Xu}$ \& Ward, 2010; Siegel, Naishadham, \& Jemal, 2013). Age is also a major risk factor since older men are at a higher risk for developing prostate cancer (Prostate Cancer Foundation [PCF], 2011). However, studies have documented that prognosis in addition 
to development of sexual dysfunction and urinary incontinence was not affected by age (Bill-Axelson et al., 2005; Sacco et al., 2006; Sanda et al., 2008). On the other hand, research has suggested that the experiences of men diagnosed with prostate cancer may differ by age (Harden, Northouse, \& Mood, 2006; Oliffe, 2005). Examining the factors that contribute to QOL in men diagnosed with prostate cancer is important especially since they are living longer with treatment side effects.

\section{Research Purpose}

The purpose of the this study was to examine the relative contributions of age, ethnicity, sexual dysfunction, urinary incontinence, masculinity, and perception of the relationship with the partner on the quality of life in men diagnosed with prostate cancer.

\section{Research Problem}

Age

There is an exponential increase in the risk of prostate cancer diagnosis with increased age (PCF, 2011; Siegel et al., 2013) and an increased risk of acquiring the disease after the age of 50 years in general. In African American men, the risk increases after the age of 40 years (PCF, 2011; Siegel et al., 2013). Around 65\% of men diagnosed with prostate cancer were 65 years and older (PCF, 2011; Siegel et al., 2013). It was estimated that $80 \%$ of men reaching 80 years old have cancer cells in their prostate (PCF, 2011; Siegel et al., 2013). However, the prognosis of prostate cancer does not differ by age at diagnosis. Studies that have examined long term outcomes and 10 years life expectancy have suggested that age was not a predictor of prostate cancer survival (BillAxelson et al., 2005; Wong et al., 2006). Nevertheless, age was found to contribute to incontinence (Sacco et al., 2006) and sexual functioning (Rondorf-Klym \& Colling, 
2003; Sanda et al., 2008). On the other hand, Stanford and colleagues (2000) suggested that age was a significant contributor to sexual and urinary functioning after the surgery in men undergoing prostatectomy. These results were also reported from a similar study that used a sample of men undergoing nerve sparing prostatectomy (Penson et al., 2005). With sexual functioning as a major concern, the effect on sexual performance of men may differ by age. The notion that sexual dysfunction does not affect older men may be the result of stereotypes that suggest that older men are expected to engage less and be less interested in sexual activities. From a qualitative study, Oliffe (2005) reported that older men were expected to have less emphasis on sexuality and therefore were reluctant to expand the discussion about the impact of decreased sexuality on their perceived masculinity. However, younger men discussed the importance of their sexuality more openly (Oliffe, 2005). Another qualitative study that explored the experiences of men with prostate cancer and their partners by age showed that younger couples had more positive perspectives of their relationship, intimacy, and changing priorities. Older men, on the other hand, focused on their perceived social roles and their family roles and discussed these roles as their main concerns (Harden et al., 2006). Despite common treatment side effects, each age group had different issues or major areas of concern regarding their relationships, intimacy, changing of priorities, and distress (Harden at al., 2006).

\section{Ethnicity}

Race and ethnicity are associated with incidence, response, and outcomes of cancer and its treatment. In the case of prostate cancer, it was reported that African American men have the highest incidence of the disease followed by Hispanic men 
(Edwards et al., 2010). Very few studies have explored differences in outcomes among different racial and ethnic groups. Stanford and colleagues (2000) identified a difference in sexual functioning among ethnicities where African American and Hispanic men reported better erectile functioning compared to Caucasian men 18 months after radical prostatectomy. Studies that have examined masculinity and partner relationships were also conducted among samples that were predominantly non-Hispanic Whites or Caucasians. Therefore, the combined concepts of masculinity, QOL, and partner relationships have not been explored with different racial or ethnic groups.

Population estimates and percentages in Miami show that a multiethnic Hispanic community includes Cuban, Columbian, Venezuelan, Haitian, other Latin American and Caribbean communities; hence, exploring these concepts in special populations or ethnic groups in a unique environment will contribute to the literature. In a Hispanic community that emphasizes the concepts of machismo and caballerismo, masculinity of men experiencing sexual dysfunction may be challenged. Machismo is a socially constructed phenomenon that emphasizes the Hispanic male gender role of being strong, a family protector and provider, and sexual prowness (i.e., sexual stamina) (Arciniega et al., 2008). The concept of machismo is closely related to hypermasculinity and traditional masculine roles (Connell, 1995). Within different cultures, the emphasis of masculine roles and sexuality may vary. Consequently, it is important to examine the QOL in men with prostate cancer in a unique multiethnic Hispanic community with respect to age, urinary incontinence, sexual dysfunction, masculinity, and partner relationship. 


\section{Prostate Cancer Treatment Side Effects}

Prostate cancer treatments vary from surgery, radiation therapy, hormonal therapy, or watchful waiting. The goal of treatment is either cure, prolong survival, or palliation depending on the staging of the tumor at diagnosis (Hoznek et al., 2005). Randomized trials conducted to compare the different treatments for prostate cancer have not suggested guidelines for which option provides better outcomes (Wei et al., 2002;

Wilt et al., 2008). Irrespective of treatment preference, the most reported side effects are sexual dysfunction (30-80\% erectile dysfunction [ED], diminished desire, and $62 \%$ impotence), urinary incontinence (30-74\% incontinence, urethral stricture), and bowel incontinence (3-10\%) (Hoznek et al., 2005; Palmer, Fogarty, Somerfield, \& Powell, 2003; Potosky et al., 2000; Sanda et al., 2008). Despite latest approaches that have attempted to use robotics or nerve sparing techniques to minimize sexual dysfunction, studies have reported minimal improvement in sexual functioning at six, 12, and 18 months post nerve sparing surgery (Penson et al., 2005). Studies have shown that sexual dysfunction and urinary incontinence were the most commonly reported side effects and have the most impact on QOL in men with prostate cancer (Penson et al., 2003; Potosky et al., 2004). Also, these side effects (i.e., sexual dysfunction and urinary incontinence) have been reported to be the most bothersome symptoms that distress QOL in men diagnosed with prostate cancer (Howlett et al., 2010; Penson et al., 2003; Sanda et al., 2008; Weber, Roberts, Chumbler, Mills, \& Algood 2007). Accordingly, this study focused on these side effects as the main symptoms of men with prostate cancer. 


\section{Masculinity}

Men with prostate cancer suffer from emotional and social burden as a result of the treatment side effects of sexual dysfunction and urinary incontinence (Wei et al., 2002). As a result of decreased sexual functioning, these men's perceptions of masculinity are affected which consequently may influence the relationships with their partners (Sanders et al., 2006). Studies on masculinity and sexual functioning of men with prostate cancer generally have used qualitative designs and have reported themes of distress, despair, shame, and impaired social functioning (Fergus et al., 2002; Khan et al., 2008). In these qualitative reports, sexual performance was reported to be an essential component of a man's socially expected role (Burns \& Mahalik, 2006). Studies have highlighted the effect of these symptoms on men's wellbeing but have not emphasized the effects on these men's relationship with their partners and QOL (Burns \& Mahalik, 2006; Fergus et al., 2002; Khan et al., 2008).

\section{Relationship with Partner}

Men and their partners are affected by the diagnosis and treatment of prostate cancer (Galbraith et al., 2008). With sexual dysfunction as a main side effect of prostate cancer, relationship issues and concerns arise. Emotional distance, decreased intimacy, emotional burden, and other struggles between couples occur (Badr \& Carmak Taylor, 2009; Hawkins et al., 2009). Studies have shown that erectile dysfunction was associated with increased conflict and decreased relationship satisfaction (McCabe \& Matic, 2008). Relationship troubles were found to be due to communication issues and perceived changes of roles of both partners (Galbraith \& Crighton, 2008; Giarelli et al., 2003). The decreased expression of emotions and experiences among men were also explained by the 
concept of masculinity. Men did not express their emotions and experiences because they did not want to be stigmatized as being weak. As a result, couples living with prostate cancer experience challenges from the time of diagnosis and throughout the treatment phase (Gray, Fitch, Phillips, Labrecque, \& Fergus, 2000). Also, the feeling of fatigue as a result of the disease and some of the treatment (i.e., radiation and hormonal therapy) prohibits men from continuing their planned activities with the partners and families which added to the relationship challenges (Sanders et al., 2006).

Communication between couples was identified as an integral component to the survival of the relationship (Badr \& Carmack Taylor, 2009). However, many couples did not have the information or guidance to manage their relationship after prostate cancer (Sanders et al., 2006).

\section{Quality of Life}

Quality of life (QOL) is an essential indicator for survival and treatment outcomes (Johnson \& Temple, 1985; Montazeri, 2009). QOL is measured in terms of social, physical, and psychological wellbeing. Men with prostate cancer generally do not look sick when compared to men with other cancers and undergo chemotherapy treatment. However, irrespective of the choice of treatment, urinary incontinence and sexual dysfunction were identified to be the main side effects of prostate cancer (Penson et al., 2003; Potosky et al., 2004). These side effects were found to have negative effects on sexual functioning which affected QOL in men with prostate cancer (Howlett et al., 2010). Monga and colleagues (2005) suggested that after the treatment of prostate cancer, time may contribute to improvement in QOL although not to baseline status. QOL outcomes were identified in the physical, psychological, and social wellbeing 
subscales of QOL instruments as well as the measures of partner distress (Sanda et al., 2008). Confirming the findings from another quantitative study, Korfage, Hak, de Koning, and Essink-Bot (2006) reported that some men rationalized that, despite the inconvenience, older age and the aging process may contribute to reaching acceptance of men's condition after the treatment of prostate cancer. In other words, older age was used by these men as a rationale that helped them reach acceptance of sexual dysfunction and/or urinary incontinence. As a result, men reported discomfort from urinary incontinence and sexual dysfunction but may not have considered their condition as a decrease in their health status because they thought that they could get used to their symptoms and were appreciative to be alive (Korfage et al., 2006). QOL studies have emphasized the impact of treatment side effects. Studies have also discussed the association between the treatment side effects and masculinity and relationship with the partner. However, studies have not expanded on the relative contributions of masculinity and relationship with the partner in addition to the treatment side effects (i.e., sexual dysfunction and urinary incontinence), age, and ethnicity on QOL in men diagnosed with prostate cancer.

\section{Significance of the Study}

Research to date has examined the concepts of masculinity, sexual dysfunction, urinary incontinence, and relationship with the partner and their independent effects on QOL in men with prostate cancer. Research has identified that sexual dysfunction and urinary incontinence impact QOL in these men (Howlett et al., 2010; Monga et al., 2005; Penson et al., 2003; Potosky et al., 2004). Qualitative studies have documented that men with prostate cancer believe that sexual dysfunction made them feel as if they were less 
of a man; however, masculinity was not quantitatively measured. The relative contributions to QOL of masculinity with other variables such as age, ethnicity, urinary incontinence, sexual dysfunction, and partner relationship has not been examined.

Relationship with the partner has been found to be affected by decreased intimacy and shift of the partner image from the lover to the caregiver (Galbraith \& Crighton, 2008; Giarelli et al., 2003). The relationship between ethnicity and QOL in men diagnosed with prostate cancer has not been extensively examined, especially in a unique multiethnic Hispanic community that is includes Cuban, Columbian, Venezuelan, Haitian, other Latin American and Caribbean communities. Nevertheless, the independent and combined contributions of age, ethnicity, sexual dysfunction, urinary incontinence, masculinity, and perception of the relationship with the partner together on QOL in men diagnosed with prostate cancer have not been reported in the literature.

This study examined the contributions of age, ethnicity, sexual dysfunction, urinary incontinence, masculinity, and perception of the relationship with the partner on QOL in men diagnosed with prostate cancer. The significance of this study is that it examined the contributions of a different combination of concepts (i.e., age, ethnicity, sexual dysfunction, urinary incontinence, masculinity, and perception of the relationship with the partner together) on QOL in men with prostate cancer from what has previously been published in the literature. With the majority of the studies conducted among White non-Hispanic samples, this study was conducted in Miami, Florida and included a sample predominantly of men who are of unique multiethnic Hispanic communities that is includes Cuban, Columbian, Venezuelan, Haitian, other Latin American and Caribbean communities. Findings of this study identified the major contributors of QOL in men 
with prostate cancer. These results will help identify areas where interventions might be developed to improve the quality of care and enhance the QOL in men with prostate cancer.

\section{Theoretical Perspective}

The theoretical perspective of this study was based on the revised Wilson and Cleary's (1995) conceptual framework on QOL (Ferrans, Zerwic, Wilbur, \& Larson, 2005). Wilson and Cleary (1995) identified health related QOL as the outcome of the quality care provided to patients. The authors defined health related QOL to include the dimensions of physical functioning, social functioning, role functioning, mental health, and general health perception. The original model presented the physiological variables, symptoms, functional status, and general health perceptions as a continuum that contributed to the overall QOL. Wislon and Cleary (1995) suggested that individual characteristics (e.g., symptom amplification, values, preferences, personality motivation) and characteristics of the environment (e.g., psychological support, social and economical support) affect the continuum dimensions (i.e., physiological variables, symptoms, functional status, and general health perceptions) and overall QOL (Wilson \& Cleary, 1995). 


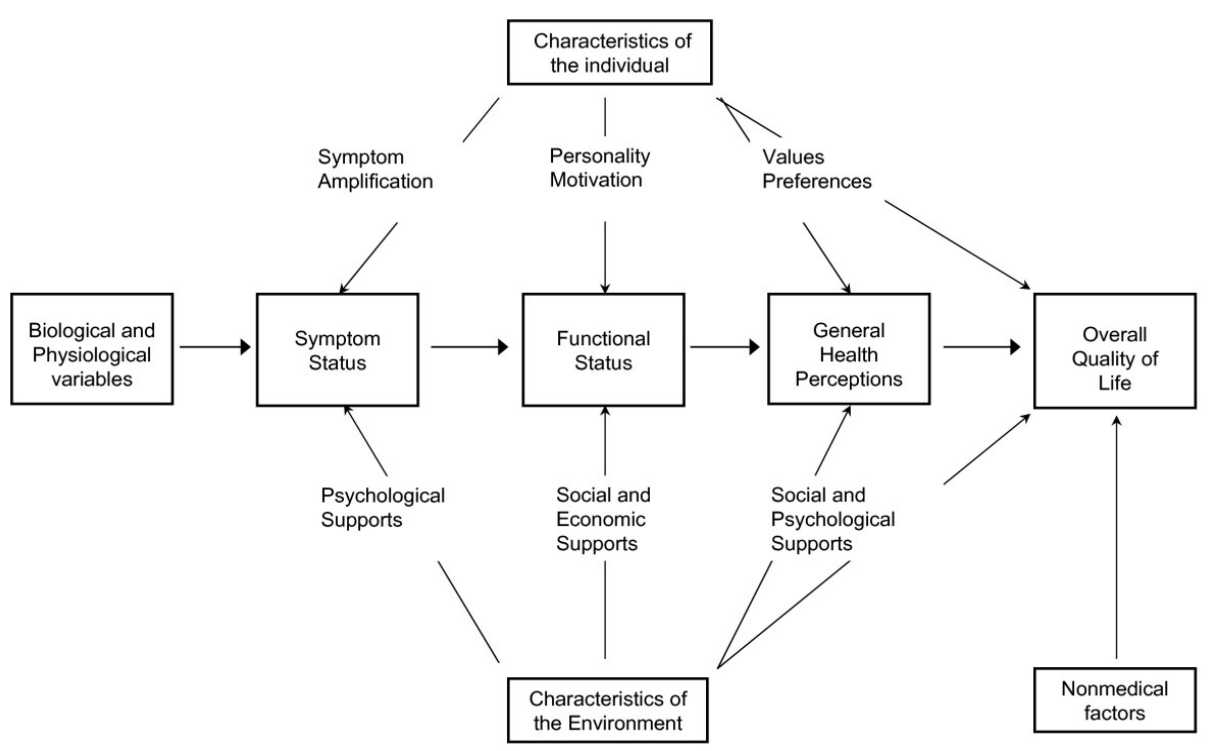

Figure 1. Wilson and Cleary's (1995) conceptual framework on QOL.

Ferrans and colleagues (2005) modified the Wilson and Cleary model and suggested supplementary explanations of the constructs of the model. The model identified health related QOL as a multidimensional concept that was determined by individual and environmental characteristics (Ferrans et al., 2005). Ferrans and colleagues (2005) further defined individual and environmental characteristics. The revised model modified the presented relationships between biological functioning and individual (i.e., demographic, developmental, psychological, and biological factors) and environmental (i.e., social or physical characteristics including interpersonal or social influences) characteristics. The revised model deleted the suggested nonmedical factors that were considered to affect quality of life (Ferrans et al., 2005). Also, the original model included labels on the relationship arrows that defined and restricted the characterization and direction of the relationship. Ferrans and colleagues (2005) 
suggested deleting these labels to facilitate the application of the model to a variety of cases.

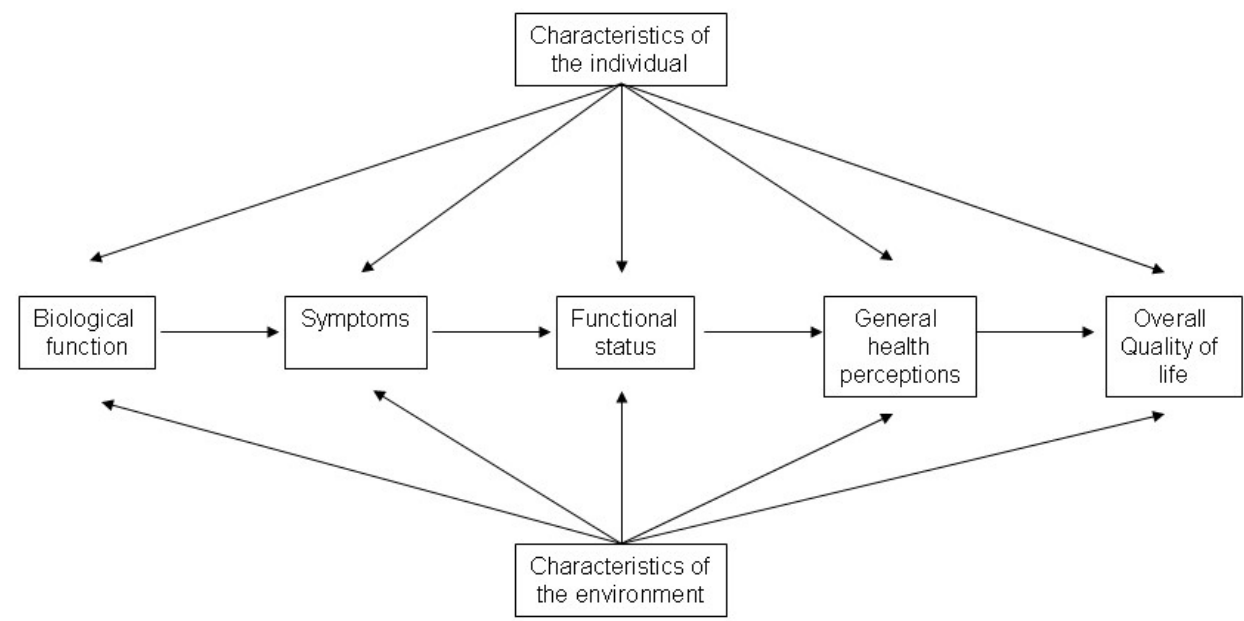

Figure 2. Ferrans et al. (2005) revised Wilson and Cleary model.

This study examined the contributions of individual (i.e., age, ethnicity), environmental (i.e., masculinity, partner relationship), and symptom characteristics (i.e., sexual dysfunction, urinary incontinence) on overall QOL in men with prostate cancer.

\section{Individual Characteristics}

Ferrans and colleagues (2005) classified individual characteristics as demographic, developmental, psychological, and biological factors that contribute to the overall QOL (Ferrans et al., 2005). With this modification, demographic characteristics such as age, gender, race/ethnicity, marital status, presence of disease, and comorbidities are recognized as influences on overall QOL. The individual or demographic variables considered as independent variables in this study were age and ethnicity. Other demographic variables such as marital status and presence of disease were not included as major study variables since the study sample only included married or partnered men diagnosed with prostate cancer. The majority of studies on the QOL in men with prostate 
cancer have not emphasized the contribution of age and ethnicity to overall QOL. However, studies have documented that age is a significant contributor to sexual and urinary functioning after the treatment of prostate cancer (Penson et al., 2005; Sacco et al., 2006; Stanford et al., 2000). The majority of research with men with prostate cancer has been conducted with samples that were predominantly Caucasian or non-Hispanic White. Few studies have included samples from ethnically diverse backgrounds to compare QOL or treatment outcomes (Sanda et al., 2008). Epidemiologic studies have documented that African American men have a higher incidence of prostate cancer, followed by White, Hispanic men (Jemal et al., 2010). However, Sanda and colleagues (2008) suggested that African American men with prostate cancer were less likely to be satisfied with treatment outcomes (e.g., urinary incontinence, sexual dysfunction).

\section{Environmental Characteristics}

Environmental factors were defined as characteristics including interpersonal or social influences on health outcomes (Ferrans et al., 2005). The influence of the social environment on healthcare has been identified to affect healthcare access and perception of healthcare outcomes (Ferrans et al., 2005). For men with prostate cancer, studies have documented that sexual dysfunction resulting from treatment led to emotional distress and distancing between couples (Hawkins et al., 2009). Sexual functioning was found to be related to relationship satisfaction; therefore, men with prostate cancer with resultant sexual dysfunction reported decreased satisfaction with their intimate relationships (Badr \& Carmack Taylor, 2009). The contribution of men's perceptions of their relationship dynamics on their QOL is not well understood. 
Men with prostate cancer may not communicate their concerns or express their emotions as explained by the concept of masculinity (Oliffe \& Thorne, 2007). Masculinity is a socially constructed that emphasizes the roles and ideologies of men (Connell, 1995; Courtney, 2000). The image of a masculine man is accentuated by the concepts of competitiveness, strength, power, and sexual prowness (Connell, 1995, Farrell, 1993; Kimmel, 2000). Sexual dysfunction constitutes a threat to masculinity and the socially expected role of men (Gray, Fitch, Fergus, Mykhalovsky, \& Church, 2002; Kelly, 2009; Oliffe, 2005). Studies that have examined the effects of prostate cancer treatment on masculinity have used qualitative research designs and have reported a great sense of shame and embarrassment expressed by men as a result of their inability to perform sexually. Their decreased sense of masculinity arises from their inability to meet the perceived socially expected roles of being a man (Chapple \& Ziebland, 2002; Fergus et al., 2002; Oliffe, 2005). Masculinity and men's perception of their relationships with their partners were considered as the environmental characteristics and independent variables since they represent the social influences on QOL in men with prostate cancer.

\section{Treatment Side Effects}

Prostate cancer treatment side effects or symptoms include sexual dysfunction (i.e., erectile dysfunction, diminished desire) and urinary incontinence (Wei et al., 2002). Studies have documented that these side effects are the main contributors to diminished QOL (Hoznek et al., 2005; Sanda et al., 2008). Also, sexual dysfunction and urinary incontinence have been identified to be the most bothersome symptoms that affect QOL in men diagnosed with prostate cancer (Howlett et al., 2010; Penson et al., 2003; Sanda et al., 2008; Weber et al., 2007). Anchored in Wilson and Cleary's (1995) model, prostate 
cancer treatment side effects were accounted for under the symptoms domain or construct. Symptoms were defined to be the patient's perceptions of abnormal functioning (Ferrans et al., 2005). However, based on the breadth of research on the contribution of sexual dysfunction and urinary incontinence on QOL, prostate cancer treatment side effects (i.e., sexual dysfunction and urinary incontinence) were identified as a separate domain or construct.

\section{Quality of Life}

QOL has been considered to be the subjective well-being or the person's perception of one's satisfaction with life (Wilson \& Cleary, 1995). The overall satisfaction with life includes several domains such as health and functioning as well as psychological, family, social, and economic wellbeing (Ferrans et al., 2005). QOL has been used as the outcome measure of disease and treatment hmm not sure what this means in healthcare (Ferrans et al., 2005; Wilson \& Cleary, 1995). QOL was considered to be the dependent variable of the study. The contributions of the demographic variables (i.e., age and ethnicity), prostate cancer treatment side effects (i.e., sexual dysfunction and urinary incontinence), and environmental factors (i.e., masculinity and men's perception of the relationship with partner) on QOL were examined. Identifying the main contributors to QOL will help in enhancing the care of men with prostate cancer. 


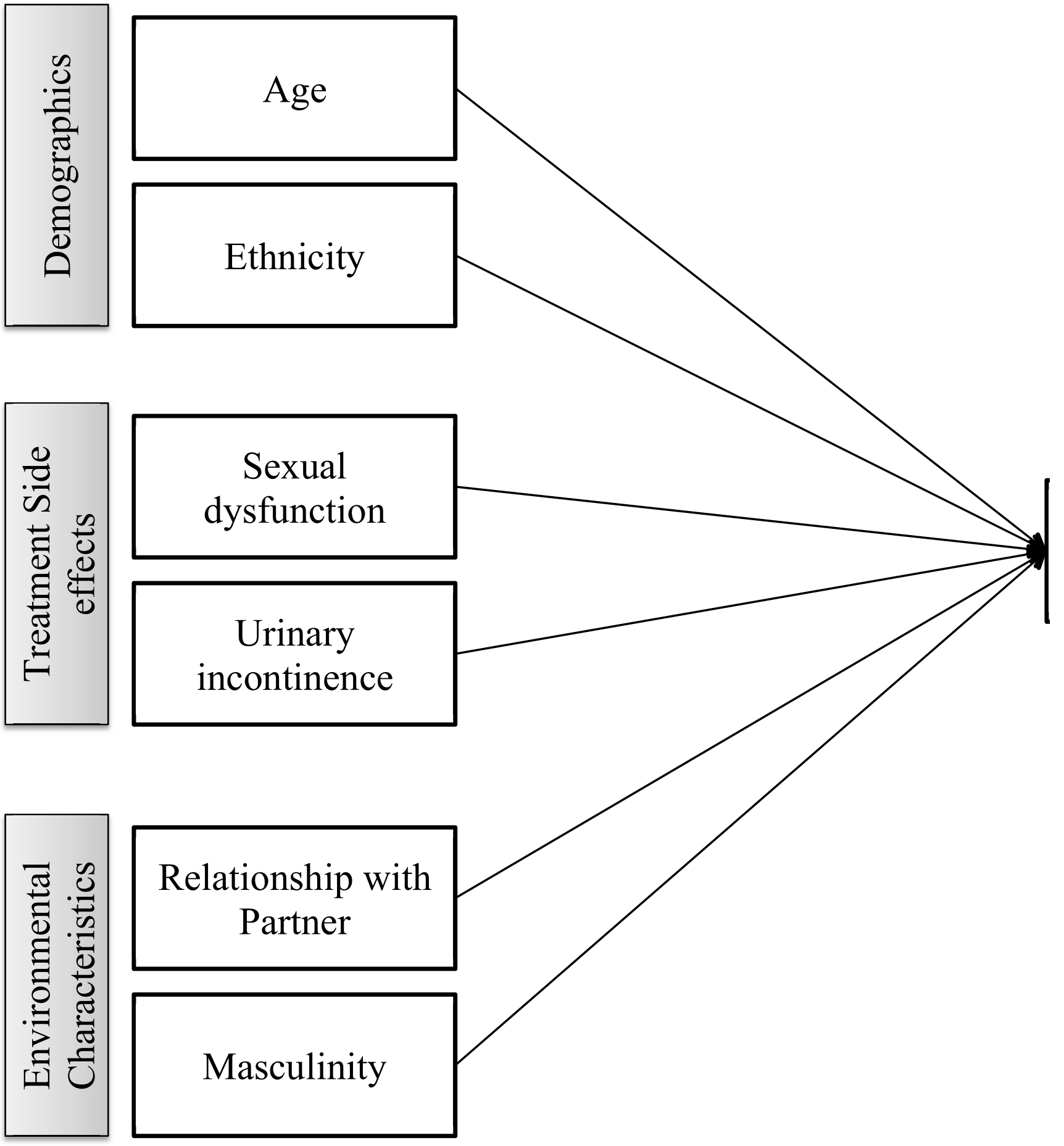

Figure 3. Conceptual framework for variables contributing toward quality of life in men with prostate cancer. 


\section{Research Questions}

This study addressed the following research questions in self identified heterosexual and married or partnered men diagnosed with prostate cancer:

1. Are there relationships among age, ethnicity, sexual dysfunction, urinary incontinence, masculinity, and perception of the relationship with the partner, and quality of life?

2. What are the relative contribution of age, ethnicity, sexual dysfunction, urinary incontinence, masculinity, and perception of the relationship with the partner to men's quality of life?

\section{Hypotheses}

The following hypotheses were examined in self-identified heterosexual and married/ or partnered men diagnosed with prostate cancer:

H1: Are there relationships between age, ethnicity, sexual dysfunction, urinary incontinence, masculinity, and perception of the relationship with the partner, and quality of life.

Age was found to be a risk factor for prostate cancer (PCF, 2011) and the consequent experience of men diagnosed with prostate cancer differed by age (Harden, Northouse, \& Mood, 2006; Oliffe, 2005) especially regarding incontinence (Sacco et al., 2006) and sexual functioning (Rondorf-Klym \& Colling, 2003; Sanda et al., 2008).

Oliffe (2005) discussed that older men were socially expected to place less emphasis on sexuality and therefore were hesitant in discussing the impact of sexual dysfunction. On the other hand, younger men placed great importance on the magnitude of maintaining sexuality in the case of prostate cancer (Oliffe, 2005). Prostate cancer treatment 
outcomes were not found to be different among racial/ ethnic groups. Sexual functioning was reported to differ by ethnic groups where African American and Hispanic men were found to have better erectile functioning as compared to Caucasian men 18 months after radical prostatectomy (Standord et al., 2000). Similar to age, studies examining QOL were conducted among samples that were predominantly non-Hispanic Whites or Caucasians. Prostate cancer treatment side effects (i.e., sexual dysfunction and urinary incontinence) were documented to distress the QOL in men diagnosed with prostate cancer (Howlett et al., 2010; Sanda et al., 2008; Penson et al., 2003; Potosky et al., 2004; Weber et al., 2007). Studies reported that sexual dysfunction was the most significant and bothersome side effect of prostate cancer treatment (Hoznek et al., 2005; Wei et al., 2002). QOL was documented to be one of the major outcomes of prostate cancer treatment, along with sexuality and the relationship with the partner (Badr, Carmak, Taylor, 2009; Giarelli et al., 2003). As a result of decreased sexual functioning, men's masculinity is affected (Sanders, Pedro, Bantum, \& Galbraith, 2006). Studies on masculinity and sexual functioning in men with prostate cancer generally have shown that men discussed main themes of distress, despair, shame, and impaired social functioning (Fergus et al.; 2002; Khan et al., 2008). Sexual performance was reported to be an essential constituent of a man's socially expected role (Burns \& Mahalik, 2006). Emotional distance, decreased intimacy, emotional burden, other struggles between couples occur after the diagnosis of prostate cancer (Badr, Carmak, \& Taylor, 2009; Hawkins et al., 2009). Erectile dysfunction was associated with increased conflict and decreased relationship satisfaction (McCabe \& Matic, 2008). Relationship troubles were 
identified to be due to communication issues and perceived changes of roles of both partners (Galbraith \& Crighton, 2008; Giarelli et al., 2003).

The impact of prostate cancer side effects on masculinity (Burns \& Mahalik, 2006; Fergus et al.; 2002), partner relationship (Galbraith et al., 2008; Sanders et al., 2006), and QOL (Howlet et al., 2010; Monga et al., 2005) have been examined. The relationships between sexual dysfunction and urinary incontinence and QOL have been well documented (Howlett et al., 2010; Sanda et al., 2008; Penson et al., 2003; Potosky et al., 2004; Weber et al., 2007). However, the relationships among age, ethnicity, masculinity, and partner relationship, and QOL combined have not been examined. Based on the reviewed literature, it was hypothesized that there was a significant relationship among age, ethnicity, sexual dysfunction, urinary incontinence, masculinity, and perception of the relationship with the partner, and QOL in men with prostate cancer.

H2: Age, ethnicity, sexual dysfunction, urinary incontinence, masculinity, and perception of the relationship with the partner independently and combined contribute to the quality of life of men diagnosed with prostate cancer.

Studies on men with prostate cancer have examined the contribution of prostate cancer side effects (i.e., sexual dysfunction and urinary incontinence) on QOL (Howlet et al., 2010; Monga et al., 2005), partner relationship (Galbraith et al., 2008; Sanders et al., 2006), and masculinity (Burns \& Mahalik, 2006; Fergus et al.; 2002). The age of men contributed to how much emphasis was placed on sexual dysfunction after the diagnosis of prostate cancer (Oliffe, 2005). Older men emphasized sexuality less than younger men (Oliffe, 2005). The impact of sexual dysfunction and urinary incontinence on the QOL in men with prostate cancer is well documented in the literature (Hoznek et al., 2005; Wei et 
al, 2002). Sexual dysfunction and urinary incontinence were found to negatively affect QOL in men (Hoznek et al., 2005; Wei et al, 2002). The socially expected role of men was closely related to sexual performance (Burns \& Mahalik, 2006). Research on the effects of sexual dysfunction after prostate cancer showed that men felt less masculine and subsequently ashamed and embarrassed (Burns \& Mahalik, 2006; Fergus et al.; 2002; Khan et al, 2008). In a Hispanic community where men pride themselves with the concepts of machismo and caballerismo, masculinity of men experiencing sexual dysfunction is a major concern (Araciniega et al., 2008). The extent to which men feel that sexual dysfunction and prostate cancer affects their masculinity and the subsequent impact on the QOL in these men was not studied. Studies have shown that prostate cancer side effects challenge the relationship of men with their female partners (Galbraith \& Crighton, 2008; Giarelli et al., 2003). However, studies have not examined how men have perceived the impact of the challenged relationship with the partner on their QOL. Accordingly, it was hypothesized that in addition to age, ethnicity, sexual dysfunction, and urinary incontinence, masculinity, and relationship with the partner also contribute to the QOL in men with prostate cancer. 


\section{CHAPTER II}

\section{LITERATURE REVIEW}

The review of prostate cancer literature conducted as a basis for this study included articles published between 2000 and 2013 from the Computerized Index of Nursing and Allied Health Literature (CINAHL), PubMed, Sociological Abstracts, PsychINFO, MedLine, Health and Psychosocial Instruments (HaPI), and Google Scholar. The key words used independently or in combination were: prostate cancer, surgery, radiation therapy, watchful waiting, active surveillance, hormonal therapy, treatment, side effects, sexual dysfunction, erectile dysfunction, urinary incontinence, masculinity, machismo, age, relationship, partner, and quality of life. A further search was conducted to obtain classic writings or research articles published prior to the identified year limits, which included essential information that was imperative to the proposed study. The databases were also used to search for theoretical or conceptual frameworks used in QOL and prostate cancer research. Selected journals were browsed for content and relevant articles (i.e., Oncology Nursing Forum, Clinical Journal of Oncology Nursing, Urologic Nursing, Men and Masculinities, Social Science and Medicine, Urology, Psychology of Men and Masculinity, Sexual and Relationship Therapy, Journal of Urology, Journal of Aging and Identity, Journal of Men's Studies, and American Journal of Men's Health). Research articles were also obtained from reviewing the reference lists in articles about men with prostate cancer.

The literature that has addressed men with prostate cancer has been widespread. This review focused on discussions about the epidemiology of prostate cancer; treatment options and side effects; masculinity, relationship with the partner; age; ethnicity; and 
QOL. These concepts constituted the independent and dependent variables in the proposed study's conceptual framework. The independent variables were age, ethnicity, urinary incontinence, sexual dysfunction, masculinity, and relationship with the partner. The dependent variable was QOL. The sections of the literature review focused on the relationship between each of the variables with men with prostate cancer.

\section{Epidemiology of Prostate Cancer}

Prostate cancer accounts for $238,590(28 \%)$ of cancer incidence and is the leading type of cancer in men (Siegel et al., 2013). In the analysis of data from 1999 to 2005, it was estimated that $92 \%$ of newly diagnosed prostate cancer cases were local or regional cancer, and the corresponding survival rate was 100\% (Jemal et al., 2010). Generally, it was estimated that one in six men will acquire prostate cancer in their lifetime (ACS, 2013; NCI, 2013). The ACS reported that 238,590 new cases were diagnosed in the United States and 29,720 deaths were due to prostate cancer in 2013 (ACS, 2013). The estimates for 2012 were 241,740 new diagnosed cases and 28,170 deaths from prostate cancer (ACS, 2012). Prostate cancer accounted for $10 \%(29,720)$ of overall cancer deaths which was the second leading cause of death in men (ACS, 2013; Siegel et al., 2013). Deaths from prostate cancer were due to the disease progression and metastasis.

In 2013 in Florida, the prostate cancer incidence was 137.7 per 100,000 and was the highest cancer incidence compared to overall rates of breast, colorectal, lung, and bladder cancer (ACS, 2013). The American Cancer Society (2013) estimated that 238,590 new cases were diagnosed with prostate cancer which was the second leading incidence after lung cancer. It was estimated that 2,200 deaths were due to prostate cancer in the state of Florida in 2013 (ACS, 2013). Prostate cancer was the third leading 
cause of death of cancer in the U U.S. (ACS, 2013). The majority of prostate cancers were localized (80\%) followed by regional (12\%), and distal (6\%) (Altekruse et al., 2010).

The incidence of prostate cancer has been increasing in past decades. The annual percentage change (APC) was significantly positive between 1975 and 1988 (2.6 APC) and 1988 and 1992 (16.5 APC) (Edwards et al., 2010). This increased incidence and diagnosis of prostate cancer was attributed to the increased awareness, prostate-specific antigen (PSA) screening, and prostate cancer biopsies (Jemal et al., 2006; Penson, Rossignol, Sartor, Scardino, \& Abenhaim, 2008).

In 1986, the Food and Drug Administration (FDA) approved the use of PSA to monitor the disease status in men diagnosed with prostate cancer (Penson et al., 2008). There was a significant decrease in APC (11.7) between 1992 and 1995 followed by a nonsignificant annual increase in APC between 1995 and 2000 (Edwards et al., 2010). This increase may be explained by the FDA's 1994 approval of the use of PSA testing for the screening of prostate cancer which may have led to the stabilization or nonsignificant increase of the incidence rates (Penson et al., 2008). The incidence of prostate cancer between 2000 and 2006 has decreased by $2.4 \%$ yearly (Jemal at al., 2010). This decrease may be attributed to the increased PSA screening and the decreased number of undiagnosed cases (Espey et al., 2007; Farwell, Linder, \& Jha, 2007; Jemal et al., 2004). Despite its contribution to the early detection of prostate cancer, some studies have debated that PSA testing did not contribute significantly to the decrease in prostate cancer specific mortality (Kohli \& Tindall, 2010). This controversy may need further research data and analysis. 
The incidence of prostate cancer varies by race and ethnicity. The 2002 to 2006 statistics showed that African Americans had the highest incidence (231.9 per 100,000) followed by Whites (146.6 per 100,000), Hispanics (131.1 per 100,000), and others (Edwards et al, 2010). These rates were similarly projected in the mortality rates of men in these populations (Jemal et al., 2010; Siegel, Naishadham, \& Jemal, 2013). Disparities may be due to variables other than race and ethnicity such as access to care, cultural preferences regarding preventive medicine, socioeconomic status, and genetic predisposition (Altekruse et al, 2010; Ward at al., 2004).

Despite being the leading cause of cancer in men, prostate cancer has optimistic survival rates. The optimism has been due to the improvement in early detection and the advancement in treatment modalities. The 5-year survival rate for prostate cancer was estimated to be $100 \%$, the 10 -year survival rate was $91 \%$, and the 15 -year survival rate was 76\% (Altekruse at al., 2010; Siegel et al., 2013). These rates have included men who have been diagnosed with and treated for prostate cancer controlling for the improvement in detection of the disease and treatment technology that may have improved the 10- and 15-years survival rates (Altekruse at al., 2010; Siegel et al., 2013). As a result, men live longer with prostate cancer and therefore live with the treatment side effects.

Due to increased survival rates, most men with prostate cancer may die from other causes not related to prostate cancer. Alternatively, some cases of prostate cancer may progress due to late detection or the dynamic evolution of the disease. Mortality rates of the most prevalent cancers showed that prostate cancer was the second leading cause of death (228,088 annually) for all age groups of men, the third leading cause of death (11,717 annually) for men ages 60 to 79 years, and the second leading cause of death 
(15,099 annually) for men ages 80 years and older (ACS, 2013; Siegel et al., 2013).

Living longer after the diagnosis of prostate cancer allows men to experience the consequences of treatment for extended periods of time and live with challenges and changes to their lives which may influence their QOL.

\section{Prostate Cancer Treatment and Side Effects}

With favorable mortality outcomes of prostate cancer, the consequences and side effects of the disease lie in treatment decisions and side effects. Prostate cancer treatments vary from surgery, radiation therapy, hormonal therapy to watchful waiting. Current evidence does not provide guidelines regarding which treatment options are favored for better outcomes (Resnick et. al., 2013; Wei et al, 2002; Wilt et al., 2008). Researchers have investigated the outcomes of treatment options independently (Hoznek et al., 2005) and in combination (Milecki, Martenka, Antczak, \& Kwais, 2010) or in comparisons with each other (Resnick et. al., 2013; Wilt et al. 2008). The goal of treatment of prostate cancer is either cure, prolonged survival, or palliation depending on the staging of the tumor at diagnosis (Hoznek et al., 2005; Monga et al., 2005). Other factors considered in treatment choice include patient's health status, age, and the rapidity

of tumor growth. Irrespective of the treatment preference, the side effects are similar and include sexual dysfunction (i.e., erectile dysfunction, diminished desire) and urinary incontinence (Wei et al, 2002). The remainder of this section will focus on the different treatments and their side effects (i.e., sexual dysfunction and urinary incontinence).

\section{Surgery}

Prostate cancer surgery is considered to be one of the curative treatment modalities. Surgery or prostatectomy (i.e., resection of the prostate gland) is an option 
for patients who are medically cleared and have minimal risk for surgery as a result of the side effects of anesthetic agents (Catalona \& Bigg, 1990; Corral \& Bahnson, 1994). This clearance is an integral consideration especially since many men diagnosed with prostate cancer are at an age at which they are at moderate to high surgical risk. The surgical procedure is preferred for localized tumors and can take a perineal (i.e., single incision in perineum) or retropubic (i.e., single incision to lower abdomen and may include lymph node dissection and nerve sparing) approach (Schuessler, Vancaillie, Reich, \& Griffith, 1991). The difference between these approaches is in the number of incisions, nerve sparing, or lymph node dissection abilities, if possible. Prostatectomy is valued for its reduction of prostate cancer mortality and decreased risk for developing consequent metastasis (Bill-Avelson et al., 2008). A laparoscopic approach may also be performed and would yield lower morbidity to the patient (Patel at al., 2004; Schuessler et al., 1991). The technologic advancements in laparoscopic procedures such as robotic prostatectomy aim to enhance surgical capabilities using the scope to be able to provide a treatment with decreased physical burden on men. Radical prostatectomy is not advised for advanced and metastatic cancers where other forms of treatment are preferred such as radiation or hormonal therapy (Fair et al., 1997; Witjes, Schulman, \& Debruyne, 1997). In a large case series study on undergoing the nerve sparing surgery, $6 \%$ of men had to use pads for urinary incontinence, and others reported dribbling after the procedure (Catalona \& Basler, 1993). Potency for men who did not have sexual problems prior to the surgery may be resumed in 40 to $65 \%$ of these men. Despite reported rates that show that most men experience at least a partial deficit (i.e., sexual dysfunction), the stage of the tumor 
and age of the patient were reported to be major determinants in preserving sexual potency (Catalona \& Basler, 1993).

The side effects of radical prostatectomy include sexual (i.e., $30-80 \%$ erectile dysfunction and impotence) and urinary dysfunction (i.e., 30-74\% incontinence, urethral stricture). These side effects have shown to impact QOL in men (Hoznek et al., 2005; Sanda et al., 2008) and are important to the morbidity of the surgical procedure itself (Sanda et al., 2008; Yao \& Lu-Yao, 1999). Latest approaches are attempting to use nerve sparing techniques to minimize sexual dysfunction.

In a population based longitudinal study, the Prostate Cancer Outcomes Study, 1,291 men diagnosed with prostate cancer who underwent radical prostatectomy were followed up for 24 months. The study examined the urinary and sexual function of men after radical prostatectomy for clinically localized prostate cancer. The sample was recruited from six different regions in the United States. The sample included a variety of ethnic/racial backgrounds (i.e., African American, White, and Hispanic). The age of men in this study ranged from 39 to 79 years. Results showed that $8.4 \%$ of men were incontinent, and $59.9 \%$ were impotent at 18 or more months after the surgery. The potency of men after the surgery varied depending on the type of surgery. In men who were potent prior to the surgery, impotence was reported to be an outcome at 18 or more months in $65.6 \%$ of nonnerve-sparing, $58.6 \%$ of unilateral, and $56.0 \%$ of bilateral nervesparing surgeries. Age was a significant contributor to sexual and urinary functioning after the surgery. Around $39 \%$ of men younger than 60 years old were potent at 18 months or more, whereas only around $15 \%$ to $21 \%$ of older men were potent at the same time. Similar findings were reported for urinary incontinence. Sexual functioning was 
found to be significantly different among races/ethnicities. At 18 or more months, $38.4 \%$ African American, 25.9\% Hispanic, and 21.3\% White men reported firm erections. Findings from this study documented the significant sexual and urinary side effects associated with radical prostatectomy (Stanford et al., 2000).

In a population based cohort study, Penson and colleagues (2005) investigated temporal changes in urinary and sexual function of men up to 5 years following radical prostatectomy. A sample of 1,288 men who were ages 39 to 79 years at diagnosis participated in the study. Data on prostate cancer treatment, medical history, urinary, and sexual function were collected using surveys at $6,12,24$, and 60 month after the diagnosis of localized prostate cancer. The majority of men in this study was younger than 65 years (56\%), nonHispanic White (74\%), and married. The results showed that $3.4 \%$ of men reported signs of urinary dysfunction at baseline. Six months after the diagnosis, frequent urinary leakage or no control was reported by $25 \%$ of men. This percentage decreased to $10.4 \%$ by 24 months which, in turn, significantly increased at 60 months after diagnosis (13.9\%). The majority of men (81\%) had erection firm enough for intercourse at baseline, whereas 9\% reported erection firm enough for intercourse at 6 months after diagnosis. The percentage of men with erection firm enough for intercourse was at $22 \%$ at 24 months and $28 \%$ at 60 months. Similarly, men reported having little or no difficulty maintaining erection at 24 months (24\%) and 60 months (30\%). The percentage change between 24 and 60 months was significantly different ( $p=$ $.003)$.Nevertheless, men reported no sexual activity at 24 months (44\%) and 60 months (46\%). The increase in sexual function between 24 and 60 months was not found to be significant. Upon review of the surgical reports of participants, men who had a bilateral 
nerve sparing surgery were found to report improved erection for intercourse at 60 months when compared to men with unilateral or nonnerve sparing surgery (40\% vs. $23 \%$, respectively). Age was a significant contributor to sexual functioning after bilateral nerve sparing prostatectomy. Around $61 \%$ of 39 to 54 year old men, $49 \%$ of 55 to 59 year old men, and $44 \%$ of 60 to 64 year old men reported erection firm enough for intercourse. Sildenafil (i.e., Viagra $\left.{ }^{\circledR}\right)$ was the most common erectile aid used $(n=520$, $43 \%)$, followed by vacuum erection device $(n=302,25 \%)$ and intracavernous injection ( $n=204,17 \%)$. When asked about the success of sexual enhancement methods, men reported that these techniques were "a lot" of help (13\%,35\%, and 28\%, respectively). Findings from this study confirmed that urinary and sexual dysfunctions were frequent up to 5 years following prostatectomy. Depending on the type of surgery (i.e., bilateral nerve sparing), postsurgery functioning can vary (Penson et al., 2005).

Sacco et al. (2006) investigated the incidence of urinary incontinence and its development over time. The study compared the effects of alternative definitions of incontinence on the incontinence rate and explored risk factors for incontinence after radical retropubic prostatectomy. A sample of 985 men with an average age of 64.5 years was followed up for 95.5 months and was assessed for urinary continence. The rate of continence was categorized by three definitions: 1 (no or occasional pad use), 2 ( 0 or 1 pad used daily for occasional dribbling only), and 3 (0-1 pads daily). Findings showed that 2 years after radical retropubic prostatectomy, men achieved continence $83 \%, 92.3 \%$, and $93.4 \%$ according to the definitions 1,2 , and 3 , respectively. The time to recover, measured by the date when patients achieved continence, was significantly lower for definition 1 in comparison with the other 2 definitions. Around $90 \%$ of men who 
complained of occasional dribbling (i.e., definition 2) considered themselves continent. Continence improved significantly with time up to 2 years after radical retropubic prostatectomy. The criteria or definitions of using pads had a significant impact on the QOL in men. Men conforming to definition 3 reported having lower QOL scores. Interestingly, the use of one pad per day was considered to be continent by some men. Age, bilateral neurovascular bundle resection, and anastomotic stricture significantly predicted incontinence (Sacco et al., 2006).

In a similar study, Lepor, Kaci, and Xue (2004) documented that continence significantly improved between 3, 6, 12, and 24 months following radical retropubic prostatectomy. One year after the surgery, $97.1 \%$ of men reported to be continent, and $97.1 \%, 94.1 \%$, and $97.1 \%$ were found to be continent according to the definitions of continence, respectively (Lepor at al., 2004).

Palmer and colleagues (2003) examined the nature, men's perceptions, and strategies used to manage prostatectomy urinary incontinence. Men were recruited from US TOO, a prostate cancer support group. The majority of the sample $(n=114)$ had a mean age of 66.4 years old $(S D=7.6)$, and a large majority were Caucasian $(95 \%)$ and married (83\%). The majority of men reported stress incontinence when lifting (72\%), coughing ( $72 \%)$, and sneezing (72\%). The men in this study used pelvic muscle exercise (54\%), pads (53\%), special undergarments (18\%), and other strategies to manage their incontinence. The perceptions of men about the important stressors related to their incontinence were also investigated. The responses were categorized into themes: degree of incontinence experienced; precipitants of incontinence; and social, emotional or physical effects of incontinence. Irrespective of the time since surgery, the majority of 
men (74\%) talked about incontinence as being a disturbing symptom that needed to be resolved. Urinary incontinence was prevalent up to 5 years following the surgery. Some men discussed the impact of urinary incontinence on their sexual relations. Around $11 \%$ of men reported incontinence when sexually aroused, attempting sex, or having sex. There were several strategies that men used to manage their incontinence: preventing incontinence, improving incontinence, anticipating leakage, and dealing with complete lack of bladder control. Vigilance and frequent voiding were the major techniques followed to prevent incontinence in addition to controlling alcohol, water, and other liquid intake. Kegel exercises were found to be successful in improving incontinence. The majority of men complained about endurance incontinence and discussed strategies they followed to anticipate leakage. Consequently, men were prepared with extra clothing or backup supplies at all times. Men with complete loss of control exerted the most effort to be able to return to their lives before surgery. Men talked about the trial and error of different procedures and techniques until they found the most appropriate method that worked for them. Most men in this study agreed that they were informed about urinary incontinence as a side effect of surgery, but they did not perceive that they had enough information to prepare them for the postoperative phase. The information they received was not detailed or precise to help them deal, accommodate, or control their incontinence. Findings from this study highlighted the experiences and perceptions of men with urinary incontinence after prostatectomy (Palmer et al., 2003). These findings are important considerations that contribute to the understanding of these men's experiences and help enhance the preoperative preparatory phase. Nevertheless, a major limitation of the study was that the type of surgery performed was not indicated. This is 
important to consider in light of the advancement in surgical techniques that are showing improved postoperative outcomes. The sample was recruited from a prostate cancer support group and may not have been representative of the general population of men. The relationship between urinary incontinence and sexual functioning was also a distinctive finding reported from this study.

Meyer, Gillat, Lockyer, and Macdonagh (2003) used an erectile dysfunction quality of life measure to assess the effect of erectile dysfunction on QOL in men after radical prostatectomy. Eighty one patients undergoing nonnerve sparing radical prostatectomy) participated in the study. The majority $(n=74)$ of the men were potent before the surgery. Out of the potent men, 56 were impotent after the surgery. Men's responses showed that their QOL was significantly affected by erectile dysfunction. Around $21 \%$ of men blamed themselves for not being able to satisfy their partners and felt less of a man. Findings from this study showcased the importance of erectile functioning and sexual potency on QOL in men undergoing radical prostatectomy (Meyer et al., 2003).

Alemozaffar and colleagues (2011) examined long term erectile function following prostate cancer treatment based on individual patient and treatment characteristics. Data on pretreatment patient characteristics, sexual health-related QOL, and treatment details were collected by the Prostate Cancer Outcomes and Satisfaction With Treatment Quality Assessment and Cancer of the Prostate Strategic Urologic Research Endeavor (CaPSURE). Data $(N=1202)$ were used to predict erectile function 2 years after prostate cancer treatment. Findings suggested that $37 \%(n=368)$ of all men and $48 \%(n=335)$ of men who reported functional erections before treatment were found 
to have functional erections post-surgery. Over half $(n=531 ; 53 \%)$ of men without penile prosthesis reported using medications or other techniques for erectile dysfunction. Pretreatment sexual health-related QOL, age, serum PSA level, race/ethnicity, body mass index, and treatment details were related to functional erections 2 years after prostate cancer treatment. Logistic regression predicted erectile function 2 years after prostate cancer treatment to range from $10 \%$ or less to $70 \%$ or greater depending on the individual's pretreatment patient characteristics and treatment details. Findings from this study showed that pretreatment patient characteristics and treatment predict erectile functioning 2 years after prostate cancer treatment (Alemozaffar et al., 2011). Joseph, Vincente, Madeb, and Patel (2005) compared the outcomes of pure laparoscopic radical prostatectomy with robotic assisted radical prostatectomy in 100 patients with localized prostate cancer. Findings showed that there were no significant differences between both procedures in disease outcomes such as positive margins and biochemical recurrence. Comparing patient functional outcomes, there were no significant differences. Findings from this study suggested that both procedures have similar patient outcomes (Joseph et al., 2005). Interestingly, this study identified unique findings about disease outcomes and supported the emphasis of the functional outcomes of surgery as major outcome indicators. These findings suggested that the different treatments had similar health outcomes and, therefore, differed only in functional outcomes.

Studies have suggested that men may experience a shortening of the penis by an average of 1 to 2 inches after radical prostatectomy (Gontero et al., 2007; McCullough, 2008; Savoie, Kim, \& Soloway, 2003). The significance of the findings from penile 
shortening on the sexual functioning of these men is not well studied, but it may be assumed that sexual performance may be negatively affected.

Gontero and colleagues (2007) investigated penile changes after radical prostatectomy by serial penile measurements. With a sample of 126 men, penile measurement were documented at baseline (i.e., prior to the surgery) and at 3, 6, and 12 months after the surgery. The International Index of Erectile Function was used to measure erectile function. Findings showed that nerve sparing surgery and recovery of erectile function predicted the final changes in penile size (Gontero et al., 2007). In a similar study, Savoie and colleagues (2003) measured penile length before and after retropubic prostatectomy. From a sample of 124 men, findings suggested a significant difference in size of the penis after prostatectomy for flaccid, stretched, prepubic fat, and circumference measurement (Savoie et al., 2003). Findings from these studies shed light on a consequence of prostatectomy that needs to be shared with men prior to them deciding whether or not to undergo surgery. This side effect may affect men's QOL and is an area that is not very well understood.

Another side effect of radical prostatectomy is inguinal hernia. Studies have shown that 7 to $21 \%$ of men may require inguinal hernia repair around 2 years after prostate surgery (Lodding et al., 2001; Lughezzani et al.; 2010; Sun et al., 2010). These rates were found to be higher in men after radical prostatectomy as compared to prostate biopsy, transurethral resection, or open prostatectomy for a benign disease (Sun et al., 2010). Rates were also higher in men who underwent lymph node dissection or radiation therapy (Lodding et al., 2001; Lughezzani et al., 2010). Inguinal hernia is a post operative problem that was not emphasized in the literature. 
Bowel dysfunction is another side effect that may be exhibited by fecal incontinence or fecal leakage in moderate to large amounts in $10 \%$ and $4 \%$, respectively. Fecal leakage was reported by $32 \%$ of men after nerve sparing radical prostatectomy and $17 \%$ of men who underwent retropubic radical prostatectomy (Bishoff et al., 1998). Although these numbers appear to be low, their significance lies in the fact that fewer than $15 \%$ of men have discussed this side effect with their physicians (Bishoff et al., 1998). As a result, many cases may be unnoticed or undocumented. Due to lower prevalence and reporting of bowel incontinence as compared to sexual and urinary dysfunction, this side effect will not be examined in the proposed study.

Despite numerous side effects, radical prostatectomy is a widely used treatment option for localized prostate cancer. Underlying reasons or rationalization for the decision to go for surgery are not well understood. The assurance of cure may be tempting for men who fear the advancement of disease. In summary, disease outcomes have not been emphasized; however, functional outcomes have been the focus of most studies. The impact of sexual and urinary dysfunction on QOL in men after surgery is well documented in the literature.

\section{Radiation Therapy}

Radiation therapy is advised for men who have been diagnosed with localized prostate cancer stages I, II, or III (Asbell et al., 1998). Treatment can be either external (e.g., external beam radiation therapy [ERBT]) or internal (e.g., brachytherapy). The goal of radiation therapy is to irradiate cancerous cells without damaging neighboring organs. Technological advancements in radiation therapy include the emergence of new machines and modalities such as intensity modulated radiation therapy (IMRT), three 
dimensional conformal radiation therapy (3D-CRT) proton beam therapy, and neutron beam therapy. Unlike other cancers, the use of radiation therapy as a prophylactic treatment for uninvolved lymph nodes does not enhance the survival of men with prostate cancer (Forman, Order, Zinreich, Lee, \& Wharam, 1986). Radiation may also be the treatment of choice for men who are not medically cleared for surgical intervention (Forman et al., 1986). Interestingly, the outcomes of radiation therapy were found to be positively correlated with the stage of the cancer (Duncan et al., 1993). Randomized control studies that evaluated the impact of higher doses of radiation showed less recurrence of prostate cancer compared to the conventional radiation dosages (Peeters et al., 2006; Pollack et al., 2002; Zietman et al., 2005). As a result, protocols have suggested the use of higher dosages of radiation.

Talcott and colleagues (2010) investigated the long term patient reported doserelated toxicity. A QOL measure was collected with a sample $(n=280)$ of men undergoing 70.2 Gy and 79.2 Gy of photo and proton radiation for clinically localized prostate cancer. There were no significant differences in urinary, bowel, and sexual outcomes between men receiving standard dose and men receiving high dose radiation. Findings from this study suggested that treatment with higher doses of radiation does not affect treatment related side effects (Talcott et al., 2010).

Brachytherapy, a form of internal or interstitial radiation therapy, is administered to men with early stage cancer through planting a radiation seed into the prostate gland using one of two modalities: permanent low dose radiation (LDR) or temporary high dose radiation (HDR). Brachytherapy was considered to be an improvement in radiation therapy treatment (Ragde et al., 1997). Other forms of radiation therapy such as intensity 
modulated, proton beam, and cyber knife therapies are rather new; therefore, long term effects are not well understood. Similar to development in surgical techniques, techologically advanced methods of radiation therapy have been used to improve men's mortality and morbidity rates.

Radiation therapy, either in the form of brachytherapy or ERBT, was considered to be curative in nature. Main documented side effects have included sexual dysfunction; bowel and bladder irritation; and obstruction symptoms such as frequency, urgency, incontinence, and incomplete emptying of the bladder (Wei et al., 2002). External beam radiation has been shown to result in acute cystitis, proctitis, and enteritis in a short term after the treatment (Catalona \& Bigg, 1990; Hamilton et al., 2001; Hanlon et al., 1997; Lim et al., 1995; Sanda et al., 2008; Schellhammer, Jordan, \& el-Mahdi, 1986). Despite these conditions being reversible and considered to be temporary effects, additional interventions were needed for longer periods of time in a few cases. Sexual potency was reported in some instances to be initially preserved but may decrease over time (Hamilton et al., 2001).

Hamilton et al. (2001) examined the impact of radiotherapy for prostate cancer on sexual, bowel, and urinary function. Data were collected from 497 men at 6, 12, and 24 months after the diagnosis of prostate cancer. The majority of men treated with ERBT were diagnosed between 65 and 74 years of age (80\%). Most men were White nonHispanic $(82 \%)$ and retired (70\%). Results showed that bowel dysfunction increased at 6 months and declined by 24 months reaching to a partial resolution. There was no significant change in urinary functioning between baseline and 24 months. There was a significant decline in sexual functioning at 6,12 , and 24 months. The percentage decline 
was higher at 24 months among men who reported to be potent at baseline. Findings from this study highlighted the significance of decreased sexual functioning after treatment of prostate cancer (Hamiton et al., 2001).

Zelefsky and colleagues (2002) reported the acute and late toxicity and preliminary biochemical outcomes in 772 patients with localized prostate cancer treated with IMRT. Patients reported acute symptoms including urinary symptoms $(n=217$, $28 \%$,$) , rectal toxicity (n=35,4.5 \%$, ) and rectal bleeding $(n=11,1.5 \%$,$) . Late urinary$ symptoms were reported by 72 men ( $9 \%$, grade 2$)$. Findings from this study showed that rectal toxicities with IMRT were less likely to occur compared to reported outcomes of conventional three dimensional radiotherapy techniques. Short term PSA levels after IMRT treatment were comparable with the other conventional three dimensional radiation techniques (Zelefsky et al., 2002).

Fowler, Barry, Lu-Yao, Wasson, and Bin (1996) studied QOL and possible side effects of ERBT for prostate cancer among Medicare-aged men. Patient data were obtained from the Surveillance, Epidemiology, and End Results (SEER) program, and men were contacted via mail for additional information. Results were analyzed from 621 men and compared with published data on surgical patient outcomes. Men who underwent ERBT reported using less number of pads for urinary incontinence (7\%, vs. $32 \%)$, lower rate of impotence (23\% vs. $56 \%$ ), and more bowel dysfunction ( $10 \%$ vs. 4\%) than their surgical counterparts. Interestingly, patients who underwent radiation did not perceive that they were cancer-free unlike surgical patients. Findings from this study showcase the difference in treatment side effects between surgical and radiation patient groups (Fowler et al., 1996). 
Choo and colleagues (2010) assessed the sexual functioning of men diagnosed with localized prostate cancer and treated with radiotherapy. A sample of 117 men participated in the study and with ages ranging from 42 to 79.8 years. Around $64 \%$ of men reported being sexually active prior to radiation therapy. Men with erectile dysfunction were found to be older and maintained on medications that affected potency. Men with erectile dysfunction rated the importance of being sexually active lower than men without erectile problems. After radiation therapy, the brief sexual function inventory scores of men who were sexually active without problems at baseline showed a major decline at 6,12 , and 24 months. With time, the degree of decline decreased, but there were no noted improvement in sexual functioning. This decline was associated with erectile dysfunction in addition to decreased sexual desire and ejaculation. Findings from this study supported the impact of radiation therapy on sexual functioning of men (Choo et al., 2010).

Similar to surgery, the choice for radiation as a treatment option is not understood. It might be suggested by the healthcare provider due to the absence of medical clearance for surgery. It may also be preferred as a noninvasive treatment compared to surgery. It is important to consider that radiation includes treatment appointments several days a week for 5 to 9 weeks as compared to surgery that requires one to two hospitalization days followed by the recovery period. Similar to surgery, the focus of research on radiation therapy among men with prostate cancer has been on functional and QOL outcomes of the treatment. 


\section{Hormonal Therapy}

Hormonal therapy or androgen deprivation therapy (ADT) is another form of treatment that aims to stop testosterone production and diminishes the growth of prostate cancer. It is generally used for palliation. Several approaches include bilateral orchiectomy, estrogen therapy, luteinizing hormone-releasing hormone (LHRH) antagonists (also called Gonadotropin antagonists), antiandrogens, ketaconazole, or aminogluthemide. The goal is to slow cancer growth by decreasing testosterone levels as a result of depriving cells of androgens (Kohli \& Tindall, 2010). The main side effects include hot flashes (55-80\%), gynecomastia (13-70\%), sexual dysfunction (50-100\%), weight gain, fatigue, and osteoporosis (Penson \& Litwin, 2003).

Bilateral orchiectomy is considered to be a smooth procedure that decreases testosterone levels immediately at a low cost (Catalona \& Basler, 1993). Loss of libido and impotence are the main side effects that render this procedure stressful for men (Daniell, 1997; Sanda et al., 2008). Estrogens, administered at levels that immensely decrease testosterone levels, are not often used due to their side effects of myocardial infarction, cerebral vascular accident, and pulmonary embolism. The LHRH antagonists were found to cause diabetes, cardiovascular disease, and stroke (D'Amico et al., 2007; Keating, O'Malley, Freedland, \& Smith, 2010; Keating, O'Malley, \& Smith, 2006). The side effects of androgen antagonists such as leuprolide, goserelin, and buserelin include impotence, hot flashes, and loss of libido (Levine et al., 2010). Pure antiandrogens flutamide and bicalutamide may cause additional diarrhea, breast tenderness, hot flashes, loss of libido, impotence, and nausea as reported in case reports (Soloway, Schellhammer, Smith, Chodak, \& Kennealey, 1996; Wysowski, Freiman, Tourtelot, \& 
Horton 1993). The other hormonal medications cause similar side effects and impact body image, mental health, and other related health related QOL indicators (Fowler, McNaughton Collins, Walker Corkery, Elliott, \& Barry, 2002; Kirschenbaum, 1995). Additionally, androgen deprivation may lead to osteoporosis and bone fractures (19.4\%) (Shahinian, Kuo, Freeman, Doodwin, 2005).

Mittan and colleagues (2002) examined the effects of GnRH analog treatment on bone loss and bone reabsorption in with prostate cancer. Bone mineral density of the hip and ultra distal radius and measurements were documented at baseline (i.e., before treatment) and at 6 and 12 months. Findings showed that bone mineral densitometry decreased significantly at 12 months. Bone loss was documented to be $3.3 \%$ and $5.3 \%$ at 6 and 12 months, respectively (Mittan et al., 2002).

Similarly, Greenspan and colleagues (2005) studied bone loss after the initiation of ADT. Bone mineral densitometry was assessed from the hip, wrist, total body, and spine. One year after the initiation of ADT, there was a significant bone loss at the hip, trochanter, radius, total body, and posterior spine. Men who initiated ADT within 6 months had an increase in total body fat and a reduction in total body lean mass at 12 months. Findings from this study suggest that men treated with ADT are at an increased risk for fractures. Maximum bone loss is reached at 1 year after the initiation of therapy, therefore suggesting early prevention to enhance patient outcomes and safety (Greenspan et al., 2005).

Tsai, D’Amico, Sadetsky, Chen, and Carroll (2007) studied the relationship between ADT and cardiovascular related deaths in men treated for localized prostate cancer. Data were analyzed for 1,015 patients treated with ADT, 3,262 patients treated 
with radical prostatectomy and 1,630 patients treated with radiation therapy (i.e., ERBT, brachytherapy, or cryotherapy). Competing risks regression analyses were conducted to investigate if ADT contributed to early death from cardiovascular causes controlling for age and presence of baseline cardiovascular disease. Findings suggested that ADT and age were significantly correlated with increased risks of death from cardiovascular causes in patients treated with prostatectomy (Tsai et al., 2007).

To determine the long term effects of ADT on lean body mass, muscle strength, bone mineral density, sexual function, and QOL, Basaria and colleagues (2002) conducted a cross-sectional study at a tertiary center. Men who underwent medical castration with GnRH agonists for at least 12 months, men with nonmetastatic prostate cancer who were post prostatectomy and/or radiotherapy but had not yet undergone ADT (non-ADT group), and healthy and ambulatory men (i.e., control group) were recruited. Findings from this study showed that ADT had significantly lower castrate levels of serum total testosterone, free testosterone, and oestradiol. Total body and lumbar spine, and body mineral density were significantly lower in the ADT groups. Also, the ADT group had significantly higher fat mass, reduced body strength, and lower sexual functioning. Men in the ADT group reported significantly lower sexual desire, arousal and frequency of spontaneous early morning erections. Consequently, men in the ADT group reported lower overall QOL scores especially in the physical function, role limitation, and perception of physical health domains (Basaria et al., 2002). Findings from this study showcased that men undergoing ADT have decreased sexual functioning and QOL among other symptoms up to 12 months after treatment. 
DiBlasio and colleagues (2008) examined the incidence of patient-reported erectile (ED) and sexual dysfunction after ADT treatment for prostate cancer. The study was a retrospective chart review for men receiving ADT (i.e., medical and surgical) for prostate cancer during 1989 and 2005. A total of 395 patient charts were included in the analysis and followed for 87.4 months. The majority of the sample was African American men (59\%), followed by Caucasian men (41\%). The majority of men (94.9\%) received ADT by medical castration, and 20 (5.1\%) underwent bilateral orchiectomy. Around $30 \%$ of men reported erectile dysfunction before ADT, and $70 \%$ reported new onset erectile dysfunction (DiBlasio et al., 2008). Findings from this study confirmed that ADT has a multiplicity of side effects in men with prostate cancer.

Unlike surgery and radiation, hormonal therapy may not be the first treatment of choice, especially if the side effects were explained to men. Decreasing testosterone levels to cause castration may not be a preferred option for men. Despite the lack of studies that have shown how treatment choice is made, assumptions may be made by understanding the importance of testosterone to men's sexuality and masculinity. Studies have documented that men undergoing ADT have decreased sexual functioning and QOL in additional to other symptoms such as cardiovascular side effects and osteoporosis.

\section{Cryotherapy}

Cryotherapy, also referred to as cryosurgery and cryoablation, a minimally invasive surgical technique, uses intermittent freezing followed by thawing of the prostate tissue in an attempt to destroy the cancerous cells (Robinson, Saliken, Donnelly, Barnes, \& Guyn, 1999). The projected goal of this form of surgery is to decrease the invasiveness of the procedure and lower the risks of the surgical outcomes. This 
procedure is considered for men with early stage cancers and men who are not medically cleared for radical prostatectomy. It is also effective for men who do not respond to radiation therapy as a result of the tumor being radioresistant. The long term outcomes have yet to be studied, and side effects are not well understood (Aus, Pileblad, \& Hugosson, 2002; Donnelly, Pileblad, \& Hugosson, 2002).

Anastasiadis and colleagues (2003) compared QOL and prostate associated symptoms in men after primary and salvage cryoablation for prostate cancer. One hundred thirty one men who underwent cryoablation were included in the study in two groups. Group 1 included men who had contraindications for or refused surgery, and group 2 included men after the failure of radiation therapy. Men were treated with neoadjuvant ADT before cryosurgery for 3 months. Men in the first group reported significantly better physical and social functioning. However, men in the second group reported worse sexual and urinary symptoms. Sexual dysfunction in both groups was highly prevalent, $86 \%$ and $90 \%$ in the first and second groups. Urinary incontinence rates were reported to be $5.9 \%$ and $10 \%$ in group 1 and group 2 , respectively. Findings from this study demonstrated that cryotherapy had similar functional outcomes as the other conventional prostate cancer treatment options (Anastasiadis et al., 2003).

Studies have reported impotence in 47 to $100 \%$ of men who are treated with cyrotherapy (Langenhuijsen, Broers, \& Vergunst, 2009). Other complications that have been discussed include incontinence, urethral sloughing, urinary fistula or stricture, or bladder neck obstruction (Shelley, Wilt, Coles, \& Mason, 2007). Due to recent development of this treatment option, these complications have been mainly reported 
through case series studies. Further examination of the complications of this procedure is needed through future studies.

\section{Watchful Waiting}

Watchful waiting or active surveillance is also considered to be a form of treatment that includes vigilant monitoring of the cancer through PSA testing and prostate biopsy to observe the disease progression. Men diagnosed with localized prostate cancer early may choose to reduce morbidity and delay treatment until disease progression is identified (Lu-Yao et al., 2009; Parker, 2004; Patel et al., 2004). Watchful waiting may be a choice for men diagnosed at a later age or men who are asymptomatic (Chodak et al., 1994; Lu-Yao et al., 2009; Whitmore, 1994).

In a longitudinal study, Waaler and Stenwig (1993) followed a small cohort of men with localized prostate cancer and had similar findings at 4-9 years follow up. In men with localized stage $\mathrm{C}$ cancer, survival rates at 5 and 9 years for men who chose watchful waiting as a treatment were $88 \%$ and $70 \%$, respectively (Adolfsson, Rönström, Löwhagen, Carstensen, \& Hedlund, 1994). These results support the decision of men who were diagnosed with localized prostate cancer to choose watchful waiting as a treatment modality. This was especially true since the risk of dying from other causes or comorbidities was revealed to be around 60\%, 58\%, and 57\% (Lu-Yao et al., 2009). Choosing watchful waiting as a treatment for prostate cancer did not negatively affect mortality and morbidity of these men.

Watchful waiting was supported by classic cohort studies on the mortality and morbidity of localized prostate cancer patients that followed up patients for around 15 years. Johansson and colleagues (1997) described the progress of untreated early stage 
prostate cancer and reported the long-term survival rates by stage, grade, and age at diagnosis. In the prospective cohort population based study, 642 men with prostate cancer were recruited. Men who died from prostate cancer and 15 year survival, corrected for cause of death other than prostate cancer were computed. Findings showed that prostate cancer was associated for $37 \%$ of deaths in men recruited for the study. Around $11 \%$ of men diagnosed with localized prostate cancer died from the disease. The corrected 15 -year survival rate (81\%) was similar in the untreated group and the group with initial treatment. The corrected 15 -year survival was $57 \%$ in men with locally advanced cancer and $6 \%$ in men with distant metastases at the time of diagnosis. Findings from this study suggested that men diagnosed with localized prostate cancer have positive outcomes when treated with watchful waiting. Radical initial treatment did not show to limit the death rate. However, for men with locally advanced or metastatic prostate cancer, treatment may improve outcomes and prognosis (Johansson et al., 1997).

In a second classic study, Adolfsson, Steineck, and Hedlund (1997) studied outcomes in men with clinically localized prostate cancer managed conservatively. In a prospective cohort study, 122 men diagnosed with localized, low-grade prostate cancer were followed up for 10 years. No active treatment was provided to $48 \%$ of men at follow-up or before death. The probability of being alive 5 and 10 years after diagnosis was $71 \%$ and $43 \%$, respectively. The prostate cancer survival rate at 10 years was $90 \%$. At 15 years follow up, $25 \%$ of men died from prostate cancer. The projected prostate cancer survival rate was $62 \%$ at 5 years. Findings from this study suggested that conservative management or watchful waiting was an appropriate option for men with a life expectancy of 10 years or less (Adolfsson et al., 1997). 
Johansson and colleagues (2004) examined the long term survival of men diagnosed with early stage prostate cancer and chose watchful waiting as their treatment. In this population based cohort study, men were followed up for 21 years. A sample of 223 men with untreated early stage prostate cancer was followed. Men whose cancer progressed were treated with hormonal therapy (i.e., orchiectomy or estrogens). Findings from this study suggested that the progression of prostate cancer was rather slow during the first 10 to 15 years. After 15 years, survival decreased. At 20 years, the progressionfree survival decreased cumulatively (from $45 \%$ to $36 \%$ ) in general. The progressionfree survival also decreased in the survival without metastases group (from $76.9 \%$ to $51.2 \%$ ), and the prostate cancer-specific survival group (from $78.7 \%$ to $54.4 \%$ ) groups. The mortality rate significantly increased from 15 per 1000 person-years (95\% CI [10, 21]) during the first 15 years to 44 per 1000 person-years after 15 years follow-up. Findings from this study showcase the long term progression of prostate cancer. Therefore, treatment with watchful waiting may not be the treatment of choice for men with an estimated life expectancy of more than 15 years. Thus, the age at diagnosis may be a significant factor for considering watchful waiting as a treatment of choice (Johansson at al., 2004).

Albertsen, Hanley, and Fine (2005) estimated the 20-year survival of men diagnosed with clinically localized prostate cancer and treated with watchful waiting or androgen withdrawal therapy alone. In this retrospective population based cohort study, 767 men 55 to 74 years of age were followed up for around 24 years. The prostate cancer mortality and mortality from other medical conditions were computed considering men's age at diagnosis and tumor grade. Findings showed that during the first 15 years, the 
prostate cancer mortality rate was 33 per 1000 person-years. After 15 years follow up, the prostate cancer mortality rate was 18 per 1000 person-years. There were no differences in the morality rates for the two follow up periods controlling for tumor histology. During the first 20 years follow up, men with low grade prostate cancer were shown to have a low risk of dying from prostate cancer. However, men with high grade prostate cancer had a high probability of dying from prostate cancer within 10 years of diagnosis (Albertsen et al., 2005). Similar to findings from previous studies, Albertsen and colleagues supported the positive outcomes of localized prostate cancer in men with a life expectancy of at least 10 years who chose watchful waiting as their treatment.

Bill-Axelson and colleagues (2008) reported the results of a randomized trial that compared the survival of men with prostate cancer who underwent radical prostatectomy $(n=347)$ and watchful waiting $(n=348)$. A 10 year follow up with histopathologic review and blinded evaluation of causes of death was conducted. A total of 137 men in the surgery group and 156 men in the watchful waiting group died during the follow up period. Death at 10 years was associated with prostate cancer in $13.5 \%$ and $19.5 \%$ in the surgery and watchful waiting groups. At 12 years, death was associated with prostate cancer in $12.5 \%$ and $17.9 \%$ of men in the surgery and watchful waiting groups. Findings suggested that the incidence of death was stable in both groups after 10 years of follow up. At 12 years, $19.3 \%$ and $26 \%$ of men in the surgery and watchful waiting groups were diagnosed with distant metastases. The incidence of distal metastases did not increase after the 10 year follow up. Findings from this study suggested that watchful waiting, as a treatment for prostate cancer, has comparable long term outcomes, prostate cancer mortality, and risk of metastasis (Bill-Axelson et al., 2008). 
To examine the outcomes of localized prostate cancer treated without initial curative therapy, Lu-Yao and colleagues (2009) conducted a population-based cohort study. Men diagnosed with stage T1/T2 prostate cancer after age 65 years from the Surveillance, Epidemiology, and End Results (SEER) program were followed for approximately 8.3 years. The 10-year overall survival, prostate cancer survival, and cancer related interventions were reported. The study identified the 10-year prostate cancer mortality to be $8.3 \%, 9.1 \%$, and $25.6 \%$ for men with well-, moderately-, and poorly-differentiated tumors, respectively. The risk of dying from causes other than prostate cancer was $59.8 \%, 57.2 \%$, and $56.5 \%$ for men with well-, moderately-, and poorly-differentiated tumors. Men between the ages of 66 and 74 years diagnosed with moderately differentiated prostate cancer had a 10 year disease specific mortality of $60 \%$ to $74 \%$. There was an improvement in the survival of poorly differentiated prostate cancer when compared to earlier published studies (Lu-Yao et al., 2009). Findings from this study showed improved survival and outcomes with watchful waiting. However, the advancement in technology and awareness about prostate cancer between the studies might play a role in the enhanced findings.

Important psychological conditions described by men who choose to go through watchful waiting include anxiety, stress, and fear of unknown (Chodak \& Warren, 2006). The choice of monitoring the disease with the assumption that it has a slow progression may free men from treatment side effects, especially if they are asymptomatic. On the other hand, studies have reported that men who chose watchful waiting as their treatment of choice may also experience sexual and urinary dysfunction (Wilt et al. 2008). 


\section{Comparison of Treatment Options}

There have been few randomized control trials that have compared patients who choose to go through one approach versus another for the treatment of prostate cancer. Generally, these studies have not been longitudinal in nature (Penson et al., 2008) and have been conducted with samples with a variety of characteristics. Overall, the evidence did not yield a preference of one method over another (Penson et al., 2008). However, Resnick and colleagues (2013) published a groundbreaking report that compared longterm urinary, bowel, and sexual function after radical prostatectomy or external-beam radiation therapy. Findings showed that there were no long term differences in functional outcomes at 15 years after treatment. As a result, the choice of treatment is left to the preference of the patient and his family where the role of the healthcare provider would be to explain treatment side effects and guide the patient and his family in their decision regarding treatment option. With the varying percentages of side effects such as sexual, urinary, and bladder dysfunctions, men are faced with a dilemma of which of these symptoms are less bothersome.

With sexual dysfunction being a common side effect of prostate cancer, Siegel, Moul, Spevak, Alvord, and Costabile (2001) examined the development of erectile dysfunction in three groups of men undergoing radical prostatectomy, ERBT, and watchful waiting. A total of 802 men had sufficient information in their medical charts about their erectile functioning and were included in this study and followed up for around 53 months. The majority (56\%) of men in this study were 70 years old or younger. The sample included 190 (24\%) African American men, 563 (70\%) White men and $49(6 \%)$ Hispanic, Asian, or men of unknown ethnicity. Findings showed no 
significant difference in erectile function between men treated with radical prostatectomy $(10 \%)$ and ERBT (15\%). Men undergoing watchful waiting reported decreased risk for erectile dysfunction. Potency significantly decreased in $38 \%$ of African America men and in $51 \%$ of White men. Men were grouped into three groups: younger than 60 years, 60 to 70 years, and older than 70 years. There were no significant differences in the development of erectile dysfunction. However, men younger than 60 years who chose watchful waiting as a treatment were less likely to develop erectile dysfunction than any of the other groups. The main predictors of erectile dysfunction were clinical stage of the cancer and race in the watchful waiting and ERBT groups (Siegel et al., 2001). Findings from this study support the evidence that has suggested that irrespective of the treatment option, erectile dysfunction is a major side effect of prostate cancer treatment.

Wilt et al. (2008) presented a comparative report to evaluate the effectiveness and consequences of treatment for localized prostate cancer. Two randomized control trials (RCTs) that compared radical prostatectomy and watchful waiting showed in one study that surgery significantly reduced death from prostate cancer (10\% vs. $15 \%)$, while findings showed no statistical differences in the other study. However, these studies had small sample sizes. Findings showed common rates of sexual dysfunction among all treatments with impotence rates of 5-80\% (including studies on nerve sparing surgical approach) (Wilt et al. 2008). In an RCT in a longitudinal study in Sweden, radical prostatectomy and watchful waiting were compared; the overall mortality difference over 12 years was not significant (Bill-Axelson et al., 2008). For men younger than 65 years, the overall mortality favored prostatectomy (Bill-Axelson et al., 2005). A population based survey in the United States comparing radiation therapy and surgery showed 
differences in morbidity as a result of the procedure (Fowler et al., 1996). The differences were in the major side effects in which radiation therapy resulted in increased bowel dysfunction ( $10 \%$ vs. $3 \%$ ) and decreased urinary symptoms (7\% vs. $30 \%$ ) (Fowler et al., 1996). In an RCT, sexual dysfunction was reported to be relieved with sildenafil citrate (i.e., Viagra $\left.{ }^{\circledR}\right)(55 \%)$ as compared with the placebo group (18\%) (Incrocci, Koper, Hop, \& Slob, 2001). In a study that compared outcomes of ERBT and radical prostatectomy that controlled for specific patient characteristics, men with the former treatment had higher rates of acute prostatitis (18.7\%) and diminished bowel functioning. On the other hand, men with prostatectomy complained of higher rates for cardiopulmonary complications $(5.5 \%)$, urinary stricture $(17.4 \%)$, urinary incontinence (9.6\%), and impotence (80\%) (Potosky et al., 2000).

Recent studies have investigated the combination of treatment options. In an RCT with 213 men, there were no significant improvements in outcomes between radical prostatectomy alone and radical prostatectomy with neoadjuvant therapy (Koltz et al., 2003). Another trial that examined the effect of ERBT alone and ERBT with brachytherapy found that the latter reduced the progression of prostate cancer (Sathya et al., 2005). External beam radiation with ADT led to decreased mortality in comparison to ERBT alone, but this was at the expense of increased side effects that accompany hormonal therapy such as osteoporosis, cardiovascular disease, diabetes, and others (D'Amico et al., 2004; Milecki et al, 2010).

Crook and colleagues (2011) compared urinary, sexual, and patient satisfaction outcomes between men who underwent surgical prostatectomy and interstitial radiation intervention. Data from American College of Surgeons Oncology Group phase III 
Surgical Prostatectomy Versus Interstitial Radiation Intervention Trial were used to analyze health-related QOL outcomes on 168 men. Patients participated in a multidisciplinary session that informed men about the procedures. Men were followed up for a median of 5.2 years (range $=3.2$ to 6.5 years). Around $60 \%$ of men in this study had brachytherapy, and $39.3 \%$ had radical prostatectomy. Study findings showed no differences in bowel or hormonal domains. Men with brachytherapy had better scores in the urinary, sexual, and patient satisfaction domains (Crook et al., 2011).

In a current groundbreaking study, Resnick and colleagues (2013) compared the long-term urinary, bowel, and sexual function after radical prostatectomy or externalbeam radiation therapy. Functional status was assessed at baseline (i.e., diagnosis) and at 2, 5, and 15 years after diagnosis. Data were retrieved from the Prostate Cancer Outcomes Study that collected information from six Surveillance, Epidemiology, and End Result sites in Connecticut, Utah, New Mexico, Atlanta, Los Angeles, and Seattle. The data were reviewed for participants who were surveyed at 6 and or 12 months after the diagnosis and resulted in a sample of 3,533 men. The sample was also limited to men who were diagnosed between the ages of 55 and 74 years old, underwent a follow up survey at 2 or 5 years, and underwent either radical prostatectomy or external beam radiation. A final sample of 1,655 men was identified of whom 1.164 (70.3\%) underwent radical prostatectomy and $491(29.7 \%)$ underwent radiotherapy. The results showed that urinary incontinence was more prevalent in patients who underwent surgery as compared to the radiation therapy patients at 2 and 5 years. There were no significant differences in urinary functioning between both groups at 15 years. Likewise, sexual dysfunction was more prevalent in patients undergoing surgery at 2 and 5 years, and there were no 
significant differences at 15 years. The findings from this study are radical in showing the long term outcomes of prostate cancer treatment (Resnick et. al., 2013) in contrast to the numerous studies that have highlighted the differences in functional outcomes after the different treatment options. Findings from this study suggest that there are no long term differences in functional outcomes at 15 years after treatment. These results will contribute to the change in the decision of treatments and may encourage the emphasis on short term functional outcomes and quality of life issues.

In summary, there has not been evidence to support men in their choice for the treatment for localized prostate cancer (Penson et al., 2008; Wilt et al., 2008). Regardless of treatment choice, there is evidence that has supported the high rates of side effects, namely sexual and urinary dysfunction, with varying percentages according to the treatment of choice. The Prostate Outcomes Study reported that sexual and urinary dysfunction was found in $56 \%$ to $58 \%$ men up to 18 months or more after surgery (Stanford et al., 2000). Other studies have documented no sexual activity in $44 \%$ to $46 \%$ of men up to 60 months after surgery (Penson et al., 2005). Nerve sparing surgeries were introduced to decrease the sexual dysfunction side effect of surgery. Researchers have suggested that nerve sparing surgeries were found to decrease the risk of sexual dysfunction (Penson et al., 2005). Also, urinary incontinence was also a major problem; however, studies have suggested that men may achieve some levels of continence up to 2 years after surgery (Lepor et al., 2003; Sacco et al., 2006). Urinary incontinence was also reported to affect sexual performance (Palmer et al., 2003).

Radiation therapy also leads to sexual dysfunction; bowel and bladder irritation; and obstruction symptoms such as frequency, urgency, incontinence, and incomplete 
emptying of the bladder (Litwin et al., 1995; Wei et al, 2002). In a population based survey of Medicare recipients, men reported requiring pads for urinary incontinence (7\%) and increased bowel dysfunction with frequent bowel movement (10\%) (Fowler et al., 1996). Hamilton and colleagues (2001) reported that $43 \%$ of men had impotence as a result of treatment 2 years after radiation therapy. Impotence was also reported by other studies with rates up to $62 \%$ (Potosky et al., 2000). Also, erectile dysfunction was discussed to be a major side effect (up to $80 \%$ ) (Monga et al., 2005). On another note, radiation was found to be carcinogenic and to lead to an increased risk for bladder and rectal cancer (Abdel-Wahab, Reis, Wu, \& Duncan, 2009; Nieder, Porter, \& Soloway, 2008).

Hormonal therapy has been a palliative treatment used for men diagnosed with advanced stages of prostate cancer due to its uncomfortable side effects: hot flashes (55$80 \%$ ), gynecomastia (13-70\%), sexual dysfunction (50-100\%), weight gain, osteoporosis, cardiovascular disease, and others (Mittan et al., 2002; Penson \& Litwin, 2003; Tsai et al., 2007). Watchful waiting also been considered to be a treatment modality because of the slow progression of the disease during the first 15 years and good survival rates (Adolfsson et al., 1997; Johansson et al., 1997, 2004). Mohan and colleagues (2009) examined the expected survival of newly diagnosed men with treatment versus observation or watchful waiting. Despite the small sample $(n=170)$, findings showed that approximately $38 \%$ of men were expected to survive more than 10 years with observation and therefore opted to choose watchful waiting as a treatment (Mohan et al., 2009). 
Research in this area is growing, and an increased number of RTCs are emerging that compare treatment modalities and their impact on mortality, morbidity, and health related QOL (Penso et al., 2008; Wilt et al., 2008). There are also new treatment protocols and modalities being investigated that aim to enhance outcomes for men with prostate cancer such as cryotherapy (Shelley et al., 2007). On another note, most studies still have not controlled for the stage of the disease, age, race/ethnicity, and other social variables on mortality, morbidity, and health related QOL. Very few studies have examined age as a variable even though age was found to affect the sexual and urinary outcomes of prostate surgery (Stanford et al., 2000).

The majority of these studies were based on large national database data or on data from large research oriented urology clinics. Information about the instruments used to measure sexual dysfunction, urinary incontinence, and other variables were not provided in the majority of the study reports. However, the majority of the studies have confirmed the high risk of sexual dysfunction and urinary incontinence as a result of the treatment of prostate cancer. As a result, QOL in men with prostate cancer will be affected.

\section{Economic Perspective of Prostate Cancer}

The economic burden of cancer includes the direct and indirect costs. Direct costs include those incurred by physician and healthcare professional fees, hospitalization, medications, laboratory services, and others. Indirect costs include those acquired by the inability to be economically productive as a result of the inability to work or early death (Grover, Zowall, Coupal, \& Krahn, 1999). The national cost of cancer care in 2010 was estimated to be $\$ 124.57$ billion with $\$ 11.85$ billion used for prostate cancer. It is worth 
noting that the impact of these costs is enormous especially with the estimated $27 \%$ increase in the next 10 years (Mariotto, Yabroff, Shao, Feue, \& Brown, 2010). The annual productivity cost from cancer was $\$ 115.8$ billion in 2000 with an estimate of $\$ 147.6$ billion in 2020. Men are reported to have a higher productivity cost than women that may be explained by the higher death rate, higher labor force participation, and higher wages (Bradley et al., 2008). These different approaches in analyzing the financial burden of cancer show different aspects of the issues faced by patients (Ramsey, 2008). In an analysis of cost in terms of days lost from work, Yabroff and collegues (2004) found that prostate cancer has the lowest lost job productivity (21.6\%), inability to work (12.1\%), limitation of work (18.9\%), days lost from work (9\%) compared to breast, colorectal, and other cancers.

Examinations of the costs of prostate cancer in specific reports do not clearly differentiate whether the disease is localized or metastasized and the consequent the consequent cost burden. The majority of cost related studies have discussed treatment specific expenses. Few studies have compared treatment costs including initial and long term financial burdens. Wilson and colleagues (2007) showed that the initial 6 month costs of prostate cancer were on average $\$ 11,495$ (i.e., from $\$ 2,586$ for watchful waiting to $\$ 24,204$ for external beam radiation). The cost decreases after the initial 6 months to an average of $\$ 3,044$. The cost in the case of prostate cancer is directly related to the selected treatment option.

The 5 year cost analysis showed that watchful waiting has the lowest financial burden of $\$ 9,130$, followed by radiation $(\$ 15,589)$, surgery $(\$ 19,214)$, hormone and radiation combined $(\$ 25,097)$, and hormonal therapy $(\$ 26,896)$. The initial cost on the 
other hand was lowest for watchful waiting $(\$ 4,270)$, followed by surgery $(\$ 15,197)$, and hormone and radiation combined $(\$ 17,474)$. The initial inpatient costs were higher for surgery $(\$ 8,765)$ and lowest for watchful waiting $(\$ 2,288)$. The initial outpatient costs were highest for hormone and radiation combined $(\$ 16,656)$. Analyzing these costs, there was a significant difference between initial and long term cost in addition to difference between inpatient and outpatient cost depending on the treatment option (Snyder et al, 2010). Studies that have explored recent technological advancement also present a financial perspective. Barbash and Glied (2010) showed that robotic assisted surgeries incur a high cost as a result of the price of the equipment, maintenance, and the demand of additional consumables such as single use appliances. For example, the cost for radical prostatectomy without and with robotics is $\$ 2,200$ versus $\$ 4,800$ (Barbash \& Glied, 2010). As a result, Medicare and insurance companies do not reimburse for these surgeries in many instances. The benefit of the robotic surgeries includes short term recovery as compared to the regular surgery which is the main temptation for patients. As a result, reports show that there is an increased number of robotic surgeries performed for the treatment of prostate cancer (Barbash \& Glied, 2010).

The costs of prostate cancer treatment are covered by Medicare and insurers. The details of the coverage may vary between different institutions. For example, HMOs cover prostate cancer treatment when it is performed by an HMO-affiliated physician (USMD Prostate Cancer Center, n.d.). Radical prostatectomy (i.e., open, laparoscopic, or robot-assisted) is an open discussion when it comes to Medicare and insurance coverage. Radical prostatectomy is paid for by Medicare and insurers; however robotic-assisted surgery may incur additional charges that are not covered by Medicare and other insurers 
such as CIGNA, United Health, and Blue Cross Blue Shield. Radiation therapy is covered by Medicare Part A for inpatients, and Medicare Part B pays for outpatients or patients in freestanding clinics (Institute for Clinical \& Economic Review, n.d.). Hormonal therapy is also covered; however, not all prescribed medications may be approved. Abarelix was approved in 2005 by the Centers for Medicare and Medicaid Services (CMS) as a substitute for GnRH agonists with and without anti-androgens in the treatment of patients with advanced prostate cancer (CMS, 2005). Cryosurgery or cryoablation is not covered by Medicare unless there is evidence that other treatment options (i.e., radiation therapy) have failed.

On the other hand, the costs of prostate cancer treatment side effects are generally not covered by insurances (Victorian, 2008). The Congress in the Department of Labor, Health, and Human Services and Education Appropriations Act of 2006 has banned Medicare and Medicaid reimbursement for ED medications. Many private insurers have followed Medicare and Medicaid's decision on decreasing or eliminating coverage of oral phosphodiesterase type 5 inhibitors, which are commonly considered as elective treatments. The American Urological Association currently supports the House Resolution 1903 on its goal to amend the Public Service Act and Employee Retirement Income Security Act of 1974. This amendment would require insurance providers to include reconstructive prosthetic urology surgery if they provide coverage for prostate cancer treatment, especially since penile implant costs were comparative to the costs of breast reconstruction and the damage to body image and sexual dysfunction due to ED is just as important when it is caused by prostate cancer (Victorian, 2008). 
Sexual dysfunction and urinary incontinence after the treatment of prostate cancer are inevitable; therefore, men opt to undergo treatments to manage these side effects. However, many of the side effect options may not be paid for by Medicare and insurers. For example, Medicare, United Health, Oxford, CIGNA, and others do not have a National Coverage Determination (NCD) for sural nerve graft to restore erectile function during radical prostatectomy (United Healthcare, 2012). Also, a collagen implant is a procedure that is used for the treatment of stress incontinence. Medicare covers the procedure when performed by men following a trauma including prostatectomy and/or radiation therapy. Moreover, pads used for urinary incontinence incur costs that are not covered by Medicare or insurers (United Healthcare, 2011).

Sexual impotency is considered to be a medical problem that may be caused by a condition and treatment. Sexual impotency is covered by Medicare and insurers. The coverage includes outpatient procedures such as external vacuum devices, pumps, or constriction rings (e.g., ErecAid), surgical treatment such as implantation of rigid, semirigid, or inflatable penile prosthesis (e.g., FlexiRod), and penile revascularization surgery. Nerve graft surgeries to restore erectile function are not covered. Medicare does not cover the electrical stimulation of the cavernous and associated parasympathetic nerves with penile plethysmography in men who underwent nerve-sparing prostatic surgery. Also, prescription and injectable medications for the treatment of erectile dysfunction are not covered (i.e., Alprostadil urethral suppository (MUSE), Testosterone patches, Caverject, etc.) (CMS, 2005). 


\section{Masculinity}

\section{Social Construction of Masculinity}

Gender is a main component of everyday life of people since birth. Gender illustrates the ways children are raised, the way family life and sexual relationships are managed, and the way people present themselves (Connell, 2010). The biological differences between males and females are accompanied by a series of cultural and societal role norms and expectations. Since birth, boys are dressed in blue and expected to be rough, tough, more demanding, and vigorous. Boys are expected to play with specific toys such as trucks, cars, construction tools, and other toys. They are nurtured to stay away from dolls and make-up kits (Connell, 2010). Starting in the family environment, gender roles are socialized. Expectations are cultured through role modeling of the father and other strong male figures such as athletes and fiction heroes. These societal expectations encourage men to behave and perform their roles by being strong, dominant, and sexually active, in addition to other characteristics that constitute the masculinity of men (Kimmel, 2000).

Masculinity, as a social construct, is influenced by societal and cultural beliefs. Discussions and writings on masculinity and the role of men in society emphasize theories of gender socialization (Connell, 1995). These gender considerations are viewed in terms of people's dynamic relationships and their social interaction. Masculinity, a relational construct, lies at the center of the understanding of gender hierarchies (Lusher \& Robins, 2010). Men are taught to be dominant and competitive, men are trained to seek leading positions in life. Connell's theory of masculinity focuses on the power exhibited by men over their female counterparts. This theory of masculinity highlights 
the strength and power of men in addition to their superiority over women and other weaker men (i.e., those who cannot comply with the strong and powerful image). These beliefs are the driving force behind most of men's interactions in society.

Masculinity is constructed as a social interaction in which boys are oriented to their social roles as they enter an organized institution (Connell, 1995). This process is thought to start during childhood when boys start playing sports. Thoughts about male hierarchies, competitiveness, ability to overcome injuries, and sexual contradictions of athletic bodies are emphasized while learning the rules of the games. Male and female children are generally treated differently by their parents (Courtney, 2000). Boys are indirectly socialized so that the norms of potency, superiority, and authority are emphasized. These ideas are further strengthened through the expected powerful and strong image of men (Connell, 1995). The expected norms and expectations grow with men and influence their views about many conditions including their health related behaviors.

Connell (1995) discussed the hierarchies of men over women and other subordinate masculinities. The dominance of men is supported by reinforcing a culture of patriarchy and superiority. The term hegemonic masculinity describes the cultural dynamics of claiming and sustaining the leading role of men in any society (Connell, 1995). The inability to meet the masculine expected role constructs an image or identity of a subordinate man. The subordinate masculinities include gay men, transgender men, or men who are underachieving (e.g., academically). In other words, men are not only viewed as being superior to women but they are also at a higher rank than other men who do not comply with the socially expected role of being masculine. With the emphasis on 
sexual performance as one of the major masculine strengths (Connell, 1995; Farrell, 1993), men with decreased ability for sexual performance may also be viewed as weak or subordinate men.

Men are taught to seek independence and to be more instrumental and taskoriented (Kimmel, 2000). These teachings define how men manage their experiences of friendship, love, and intimacy. Stemming from their practical perspective, men show that they rely on rational, practical sex to express their intimacy in relationships (Kimmel, 2000). Accordingly, sexual relationships are gendered, and sexuality has been masculanized. The masculinization of sex includes the emphasis on the sexual activity, multiple partners, the ability to satisfy the partner, and sexual experimentation (Kimmel, 2000). Men accentuate the importance of sexual encounter to satisfy their role expectation; therefore, sex is viewed as a contest in which most men feel obliged to dominate.

Men are brought up and nurtured into these norms throughout their lives. The inability to meet these expectations contributes to negative feedback that ranges from frowns to disappointments (Connell, 2010). When the inability to meet socially constructed norms is due to a health condition, disease, or treatment, men feel disappointed and react in a manner that initially impacts their immediate environment such as their QOL.

Considering the social constructionist viewpoint, masculinity is influenced by society, history, social class, and culture (Courtney, 2000). Accordingly, the ramification of illness or disability among men may lead to reducing their gender hierarchal status that may contribute to a shift of power (Charmaz, 1995). Thus, with a diagnosis of an illness, 
men may perceive some loss of their power that challenges their perceived image as a man. In the case of prostate cancer and treatment side effects (i.e., sexual dysfunction and urinary incontinence), there is an added concern with the decreased sexual performance that may challenge their perceived role expectation. This concern arises from the increased emphasis on the ability of men to satisfy women sexually (Gray et al., 2002; Khan et al, 2008).

With the superior and strong socially constructed norms, men are cultured to shape their behaviors accordingly. These traits can be seen in the communication style differences between men and women (Connel, 2010; Kimmel, 2000; Oliffe, 2005). Women exhibit more expressive communication patterns whereas men have more assertive, goal oriented, and dominant patterns (Connel 1995; Connel, 2010, Courtney, 2000; Kimmel., 2000). As such, men do not express their feelings and refrain from discussing issues that represent areas of weakness and subordination (i.e., prostate cancer diagnosis and treatment side effects) (Hoyt et al., 2013). With these communication differences, it is expected that when a man is diagnosed with a illness condition, the communication between the couple is challenged as a result of the different styles utilized.

In the majority of qualitative studies that have included men with prostate cancer, a main theme related to the inability to perform sexually and its effect on their ability to view themselves as masculine arises and results in a shift in men's perceived status (Gray et al., 2002; Khan et al., 2008). This change in status, therefore, is a major stressor for many men. Men define their masculinity in terms of abiding by the historic social 
construction of being strong, powerful, and superior to women in their environment that is emphasized by their ability to sexually perform and satisfy women.

Several behaviors or activities may contribute to the concepts of stoicism and sexual proficiency or potency. These concepts inform the masculinity principles and are viewed in terms of being successful, capable, reliable, and in control (Cheng, 2008). Sexuality and sexual performance are considered to be indicators that confirm the gender role of men and their perceived masculinity (Kimmel, 1990). In cases in which there is an inability to sexually perform, men believe that they are pressured to perform their expected role (Oliffe, 2005). Again, prostate cancer and treatment side effects may cause a threat to one of men's expected roles. The sexual role expectation was emphasized by men in qualitative findings from Gray and colleagues (2002), Khan and colleagues (2008), and others (Duncan \& Dowseit, 2010; Hawkins et al., 2009). Detailed discussions about these concepts will be elaborated in the following sections.

\section{Masculinity and Health Behaviors}

Understanding the social construction of norms of gender roles is imperative in understanding men's health behaviors. People, in general, are influenced by identified "normal" behavior to guide their responses to what they believe their social environment accepts or identifies as deviant (Cialdini \& Trost, 1999). Normative behaviors are acquired from the cultured perception of the image of men. Normative male figures are nurtured through role models in the society and introduced through the immediate environment and social media. The "heroic" man is pictured to be strong, a risk taker, not needing medical attention, and sexually appealing and active. The perceptions of men's expected behavior are strong indicators of their responses to health issues and 
behaviors (Addis \& Mahalik, 2003; Mahalik, Burns, \& Syzdeck, 2007). The majority of studies on the social influences on health behaviors were conducted among young adults and investigated risky behaviors such as smoking (Weiss \& Garbanati, 2006), alcohol consumption and driving (Babington, Kelley, \& Patsdaughter, 2007; Linkenbach \& Perkins, 2006), and risky sexual behaviors (Babington, Kelley, \& Patsdaughter, 2009; Patsdaughter, Kelley, Babington, \& Dyer, 2005; Peterson \& Bakeman, 2006).

Several studies have examined the impact of social norms and masculinity on health promotion and health seeking behaviors (Addis \& Mahalik, 2003; Mahalik et al., 2007; Pleck, Sonenstein, \& Ku, 1994). Seeking medical help is viewed by men as a form of weakness, and they reportedly preferred to deal with their symptoms and learn to live with them (Solomon, Greenberg, \& Psyszczynski, 1991). This reluctance in seeking medical attention is especially true when symptoms are considered to be embarrassing and shameful such as urinary incontinence and sexual dysfunction (Chapple \& Ziebland, 2002; Gray et al., 2002).

O'Brien, Hunt, and Hart (2005) studied the experiences of help seeking behaviors and the links or relationships with practices of masculinity using 14 focus groups. The study included 55 men with an age range of 15 to 72 years. The men were from diverse occupational statuses, socioeconomic background, and health statuses. Men in this study complained of various conditions including prostate cancer, coronary heart disease, and mental health problems. Discussions during the focus groups were facilitated using general questions about discussing health issues with other men and seeking medical help. There was minimal input about masculinity from the facilitator because the aim was to allow it to emerge from the men's discussions. During the focus groups, there 
were differences in the responses between younger healthy men and older men with cardiac and mental health problems. Participants who had not experienced a serious illness expressed their unwillingness to consult a physician for minor complaints. They discussed that seeking medical help would be for a really serious condition or if something was really wrong. Older men in this group discussed the pressure by their wives to seek medical help for persisting symptoms. These men required reassurance that they were not making an issue about a trivial problem before they actually sought professional health care. Men who had a medical treatment due to a cardiac emergency explained that they did not seek medical help prior to their accident. These men discussed that during their cardiac emergency, they still were debating if their symptoms were serious enough to seek medical attention. Men waited before they actually received treatment because they hoped that the symptoms would probably be resolved with time. Seeking help for a mental health condition was a challenge to the men's masculinity. Men were hesitant to discuss mental health issues and referred to them as emotional disturbances or stress without elaborating or defining the problems. Some men were even resistant to explore personal experiences of depression or depressed mood. Men who were able to discuss issues related to depression maintained their masculine image and sense of control in their scripts by discussing their struggle with stress or distress and their inability to cope alone. Men with prostate cancer in this study were in the advanced stages of the disease and were using watchful waiting as a treatment. Their discussions indicated that they were proactive in seeking medical attention. Since these men were on watchful waiting as a treatment, they were expected to continuously monitor changes and the progress of the disease. Their health seeking behaviors only included practices to 
monitor their cancer. These men also recognized the "macho" aspects of not coming forward for medical help as exhibited by other men. Overall findings showed the repetitive reluctance to seek medical help since men explained it to be challenging to their strong masculine image. Men talked about enduring symptoms until tangible physical and visible symptoms were evident before attempting to seek help. The influence of others, especially female partners, to pressure men to seek help was also evident in the discussions of the men (O'Brien et al., 2005). Findings from this study emphasized the barriers related to the perceived masculine norms of men to seek medical help. The sample included different groups of men with a wide range of experiences. Although there were common concepts among the groups, there were also differences in the manner of expressing their health seeking behaviors. This study introduced the influence of the male gender in health seeking behaviors.

Using retrospective life course methods, Oliffe (2009) described the relationship between health behaviors and masculinity across time among three Anglo-Australian men recruited from prostate cancer support groups. The author used a social constructionist gendered framework for the analysis of the interviews and findings. The results were presented in chronologic phases of life. The early years had the theme of ill-boys in which being healthy was a social and self-expectation despite the presence of illnesses from time to time. The three men emphasized how social expectations influenced their actions regarding healthcare. The authority of the female as a primary healthcare provider was apparent. The focus of healthcare was for treatment of a problem and definitely not for preventative care. With the expectation to be healthy, the presence of an illness was initially treated with compulsive behaviors and private problem solving 
which is reflective of being in denial. During the middle life phase, there was an intersection of work, wife, and family. In this phase, the relationship between social status and gender was dominant and revealed working class masculinity characteristics and emphasis of the breadwinner role. Therefore, functionality and physical performance were imperative in addition to behaviors that men perceived to inform their masculinity such as smoking and drinking. Men did not show any ownership of their health as they did for their family and car. During the older age stage of life, the diagnosis of prostate cancer forced men to modify some of their health behaviors. The changes in health behaviors included adopting some healthy behaviors, following through with their health conditions, and accepting to talk with other men about their health issues. This change in health related behaviors was evident with age, especially when illness and dysfunctions disrupted men's social expectations (Oliffe, 2009). Findings from this study highlighted the influence of masculinity, age, and social influence on the health behaviors of men across the lifespan.

McVittie and Willock (2006) examined the understanding of health, ill health, and the relationship between them and the implications for masculine identities. Twelve semistructured interviews were conducted with men in Scotland ages 65 years and over. Findings showed that men valued being healthy which entailed the ability to do anything they wanted without restrictions. The men emphasized the importance of being able to control their lives and their health which was reflective of the hegemonic masculinity traits of domination and power. In discussion of the experience of ill health, participants presented illness as an external factor such as a problem or accident that was managed by an expert or physician. As such, the hegemonic masculinity was maintained despite the 
challenge of an external force. Therefore, there was a renegotiation of their identity to accept a relatively subordinate position for a specific time. The time rationalization allowed for the transition from a powerful masculine image to another state that included battling an external force which is the illness. The transition of the masculine image also permitted the man to bond with the subordinate masculinity (McVittie \& Willock, 2006). Findings from this study contribute to understanding the dynamics of negotiating masculinity of men when considering healthy behaviors or seeking medical attention.

For men who seek medical guidance, it is worth examining their relationships and communications with their physicians and other primary care providers. These are phenomena that are important to be considered since patients and their physicians are men most of the time. With gender considerations regarding prostate cancer, disease related discussions with healthcare providers are significant. Oliffe and Thorne (2007) examined qualitative transcripts from two studies that investigated patients' experiences with prostate cancer and explored patient-physician communications and interactions with the influence of masculinity. The sample included 29 Canadian and 33 Australian participants who were being treated by male urologists for prostate cancer. The mean age of patients was 61.73 years old $(S D=8.59)$, and the majority underwent prostate cancer treatment for a period of around 3.03 years $(S D=5.03)$. Findings included themes about the relationship between the disease and men's perceptions of the communication with their physicians. There was an overarching complexity inherent in prostate cancer communication, especially with respect to the cause or the onset of the disease. Men were found to be uncertain about their disease and its trajectory. The choice of the most appropriate treatment constituted a dilemma for men in the sample. This ambiguity 
influenced the masculine ideals that favor rationalization and problem solving conceptualization as it influenced the expected patient-physician communication scripts. The lack of definitive answers disrupted the traditional hierarchical relationship between the patient and his physician. Findings showed that the dialogue about prostate cancer screening started with a general practitioner (GP) and then continued with a specialist or urologist. This relationship was not typical to the masculine communication process between men. Nevertheless, the communication process was described to follow a businesslike transaction (i.e., straightforward process). With time, a trusting relationship was developed that conformed to the hierarchy and authoritative image of the physician. It was documented that anxiety was tempered by humor and sarcastic jokes. Communication with the GP was found to be dissatisfying in cases in which there were unexpected experiences or reluctance on behalf of the physician to explain treatmentrelated issues (i.e., expectations, prognosis, and side effects). Generally, the patientphysician relationship was found to conform to masculinity traits; therefore, some men expressed difficulty communicating their needs with their physicians. However, findings from this study showed that there were some contradictions between the masculinity patterns of behaviors and communication in some of the men's discussions about the prostate cancer communication process (Oliffe \& Thorne, 2007). Findings from this study shed light on the dynamics related to the communication between men and their physicians.

Mahalik and colleagues (2007) examined the contribution of masculinity and men's perceptions of normative male and female health behaviors in predicting men's own health behaviors. A sample of 140 Caucasian $(80.7 \%)$, married $(51.4 \%)$ or in a 
relationship (17.9\%), and university educated men identified the influence of masculinity and men's perceptions on their health behaviors. The mean age of the men in the study was 42.9 years $(S D=13.7)$. Health promotion behaviors including the frequency of engaging in health behaviors, masculinity, and perceptions of normative health behaviors were measured. Health promotion behaviors were measured using Likert scale items about eight health behaviors: alcohol consumption, applying a seatbelt, physical fighting, talking to someone when dealing with a troubling issue, having an annual physical examination, using tobacco, exercise, and eating healthy. Masculinity was measured using the Conformity to Masculinity Norms Inventory, and perceptions of normative health behaviors were measured the participants' perceptions about the previously listed health behaviors. Hierarchal regression showed that masculinity and men's normative perceptions of health behaviors significantly contributed to explaining men's health promotion behavior scores. Men with higher masculinity scores exhibited less health promotion behaviors (Mahalik et al., 2007). Perceptions of men's normative health behaviors in this study represented the social constructions of how men are expected to deal with health issues. Despite the limitations of the study that included online recruitment of a sample that was predominantly Caucasian with a wide age range from 18 to 78 years, findings allude to a significant influence of social norms and masculinity on health behaviors.

Men do not readily seek medical help and may not feel comfortable in discussing sensitive and embarrassing symptoms. The majority of studies about male health seeking behaviors have used qualitative designs and included homogeneous samples who were predominantly White non-Hispanic men. Although findings were important, they cannot 
be generalized to the overall population. It is important to ensure that the concerns of men are discussed and proper information is shared in an attempt to properly address concerns especially those that influence their QOL.

\section{Masculinity and Prostate Cancer}

Chapple and Ziebland (2002) studied the manner in which prostate cancer and its treatment affects men's bodies, their social roles, and senses of masculinity. The majority of the sample were White $(n=48)$, and more than 60 years old. The unstructured interviews with 52 men included discussions about the time of PSA testing, presence of prediagnosis symptoms, reason for seeking examination, and their experiences with the treatment and related side effects. As the theme of masculinity emerged, men were asked to further elaborate about the impact of the side effects of prostate cancer on their image as men. Findings were described according to the main topics discussed: men's health behavior and treatment side effects (i.e., incontinence, lack of energy, impotence, and other hormonal side effects). During the interviews, men expressed their reluctance to consult physicians due to the traditional norms that support the masculine identity. They felt that seeking a primary care provider or specialist was a sign of weakness that constituted a threat to their masculinity. Some men emphasized that it was embarrassing to seek help for symptoms of prostate problems. When discussing treatment side effects, men talked about the importance of being able to control the body and to act appropriately in social settings. Consequently, incontinence and other symptoms such as urinary frequency and urgency due to prostate cancer treatment suggested a lack of control of bodily functions and, therefore, were inconvenient and embarrassing. Men who underwent hormonal treatment talked about 
symptoms such as the lack of sexual desire that made them feel that they lost all masculinity. On the other hand, men who underwent external beam radiation also talked about the discomfort of diarrhea, while others discussed having decreased energy that impeded their ability to work and maintain the role of being the household breadwinner. The lack of energy was a concern, although a majority of men continued working during treatment or returned to work soon afterward. As a result, men in this sample were not able to conform to their perceived and expected social roles. A few men talked about being forced to retire early and felt that they lost part of their masculine image and ability to work. All except one of the men reported limited sexual functioning and discussed trying medications and other techniques to deal with the erectile dysfunction. Some men were embarrassed to discuss their sexual dysfunction and possible treatments with their physicians and wife. Their lack of ability to maintain an erection was their main issue and disappointment. Alternately, some men were appreciative of being cured of prostate cancer and considered impotence to be a price for survival. A grateful attitude was found in older men who discussed that they could handle impotence and suggested that it would be a bigger problem for younger men. Findings from this study highlighted the importance of the ability to perform sexually on masculine identity of men despite the fact that some men considered it as a small price to pay (Chapple \& Ziebland, 2002). Chapple and Ziebland (2002) discussed the concerns of some men regarding their sexuality as being the result of awareness about medical regimens and other treatment options.

In a study that explored the experiences of men living with sexual dysfunction as a consequence of having been treated for prostate cancer, 18 men (i.e., 14 heterosexual 
and 4 homosexual) were interviewed in a series of four to five sessions each. The majority of the men were White and recruited from a prostate cancer support group. The participants were 57 to 75 years old and lived with prostate cancer diagnosis for 1 to 8.5 years. The majority of men in this study $(n=13)$ reported minimal to no erectile function after their treatment. Semistructured interviews were conducted. Interviews discussed the experiences with prostate cancer in addition to dialogues about the men's childhood, important relationships, family life, their values and beliefs about the meaning of being a man, and the impact of prostate cancer on these values. Findings documented that loss of sexual functioning was a major challenge to men's masculinity. Men considered that the focus of their adjustment included treatment or prevention of sexual dysfunction. Accordingly, the investigators identified "preservation of manhood" as an important overarching theme that described participants' experience with prostate cancer. Findings identified five themes: enhancing the odds, disrupting a core performance, bearing an invisible stigma, effortful mechanical sex, and working around the loss. The majority of men in the study considered prostate cancer treatment as a tradeoff for life, although the sexual sacrifice was not as simple as they anticipated. Men in this study varied in their knowledge and confidence in the availability of options to predict or prevent decline from their previous sexual functioning levels. Sexual performance was an integral expression of manhood, and men felt pressured to maintain the social expectations of being able to sexually perform. Some men talked about the ability of having sex in terms of virility and vitality. Irrespective of the sexual orientation of the men, erectile function was considered to be a conquest and competition in which a dysfunction meant that the man lost the ability to relate to other men. Men with erectile dysfunction were reluctant to 
discuss their concerns because they considered that sexual impairment constituted a threat to who they were. They perceived that they could not participate in being vocal about their sexual accomplishments. In a culture and environment that supported sexual competition, these men perceived that they had to hide their dark secret as an invisible stigma. As long as they did not come out or disclose their problem, they felt secure and safe from socially constructed shame and embarrassment. The majority of men in the study discussed reliance on mechanical devices such as vacuum pumps, intracavernosal injections, or other mechanisms to restore their sexual functioning. These devices were found to disrupt the natural flow of sexual intercourse and, therefore, added a strain and lack of spontaneity associated with sexual performance. Some men described their sexuality as a switch that was turned off by the treatment of prostate cancer. In an attempt to regain their sexuality and manhood, some men tried to find ways to work around the loss. Findings from this study were presented from a theoretical framework that showed the experience of older men whose sexual dysfunction was principally due to prostate cancer treatment (Fergus et al., 2002).

In a qualitative study, Gray and colleagues (2002) explored the relationship between masculinity and prostate cancer. A narrative approach was used that focused on stories about the lives of men with prostate cancer. In a sample of 18 men from the larger Toronto area, four interviews (i.e., introductory meeting, prostate cancer experience, life prior to prostate cancer, and the link between precancer and postcancer lives) were conducted with the participants. The sample included four gay men and four AfroCanadian men. The investigators intentionally included men with different social experiences to allow the study to explore the differential construction of masculinity with 
different sexual orientation and ethnicity. Findings from a streamlined approach to narrative display shed light on the different perspectives of hegemonic masculinity and prostate cancer. The experiences of the men shared core elements: work experiences, relationships with women, sexuality issues, relationships with other men, prostate cancer self-help groups, health, illness and the body, age, and renegotiating masculinity. Work experience shaped the manner in how men approached the disease and the decision on treatment. Men in this study identified their wives as their source of social support, but some of them did not go to their wife for expressing emotions and feelings about their sexual dysfunction and urinary incontinence. Relationship with the wife differed between the different men in this study, and there were various approaches to how they interacted with and allowed the wife to support them. For example, some men allowed their wives to go with them to their appointments and be involved in discussions about sexual dysfunction. All of the men expressed that their sexuality was their primary concern, and some of them talked about preferring a more serious disease with which they could deal better with its consequences. Men also discussed that prostate cancer interfered with their masculinity and ability to have multiple partners. Sexual activity was a major indicator of their ability to express being a man. Some of the men thought that the importance of being sexually active is to be able to meet the needs of their wives and live up to their perceived sense of masculinity. The report of findings from the study did not include the experiences of gay men. One of the men narrated that after the diagnosis of prostate cancer, he started flirting with more women to feel appealing and be acknowledged. Interestingly, all of the men stressed that they did not want other men to know about their prostate cancer diagnosis and sexual issues. They were ready to discuss 
their concerns with female colleagues but did not want to show other men their struggles because they perceived confession as losing a sense of control. Unlike findings from other studies, men in this study were ready to go to support groups and were involved in health promotion activities. Age and aging of the body were not discussed by the men, and this omission may have been because men in the sample were generally younger than the average age of men with prostate cancer. Hegemonic masculinity was evident in the transcripts of men in the precancer and postcancer discussions. However, they renegotiated some of their behaviors and changed some of their perceptions in an attempt to maintain their perceived masculine performance. Most of the changes involved modifications in their relationships with their wives which included refraining from extramarital affairs or viewing wives as administrative assistants helping them with the consequences of the disease. There was a shift in the social relations described by these men which was accompanied by a shift in the meanings of masculinity toward a new social environment territory. The men's experiences during the precancer phase influenced their behaviors in the postcancer phase (Gray et al., 2002). Findings from this study highlight the importance of exploring the change in the meanings of masculinity and its influence on men's behaviors. The inclusion of men with different sexual orientations and ethnicities gave an overview of the experience of different men, but it might have also concealed the details of the experiences of these men within their culture. However, the experiences of men with different sexual orientations were not discussed and expanded by the authors. Also, the authors did not discuss the differences in narratives between men of different ethnicities. The article focused on the narratives of 
three White heterosexual men. The other men interviewed were not demographically identified by the authors.

Studies of men with prostate cancer that have addressed masculinity revolved around the masculine social role expectations and challenged sexual functioning. In a qualitative study using an ethnographic approach with 15 men who underwent prostatectomy, Oliffe (2005) found main concerns to be shortened penis, loss of potency, and reactions to impotence that revolve around treatment for the erectile dysfunction. The age of the men ranged from 46 to $74(M=57.06, S D=6.68)$. All of the men were in a partnered relationship for an average of 27.6 years $(S D=12.99)$. Findings documented three major themes: anticipation of impotence, acute recovery, and reactions to impotence. Upon diagnosis of prostate cancer, men were prepared to trade their potency for the potentially life threatening illness, functionality, and longetivity of relationships. Prior to the surgery, survivorship was the main concern of these men and their wives. Some men needed reassurance from their wives about their sexuality to satisfy their hegemonic masculinity and to make sure that they would not be viewed as "a lesser man.” Older men who experienced challenges with their libido and sexual prowness expressed their acceptance of the possibility of impotence following the surgery. The discussions of men referred to the social construction of asexual older men and the "filthiness" of being sexually active. Men displayed a hierarchy with the expectation of younger men to express their sexuality openly, while older men were expected to place less weight on sexuality. During the recovery phase, men discussed shortening of the penis. This consequence was a major issue since penis size informed the masculine hierarchy between men and differentiated between men and women. Loss 
of potency was also a concern since it led to emotional challenges and added to the decrease in sexual performance. During the interviews, men referred to media that mocked male impotence. They also talked about the external and internal pressures to sexually perform according the normative ways (i.e., penetrative sex). As a reaction to impotence, many men tried to treat their erectile dysfunction with medications and other procedures. However, men reported that with time they stopped using these treatments because they were artificial, ineffective, and lacked spontaneity in achieving and maintaining erection. The concept of redefined masculinity included discussions about the preference of treatment to restore their potency. Findings from this study highlighted the redefined sexuality and masculinity experienced by men following prostatectomy (Oliffe, 2005). Findings supported the socially constructed concepts of hegemonic masculinity. This study was conducted with Canadian men and did not include ethnically diverse populations.

Kelly (2009) examined masculine embodiment in the context of prostate cancer. Using an ethnographic approach, interviews with 14 men and observations at significant events such as diagnostic consultations and radiotherapy were conducted over a period of 18 months. Five professionals working with these men were also interviewed. Themes that emerged represented the trajectory of the cancer experience: "physical change and living with a new body", "diagnosis", "restoring the masculine self", "working with changed men", "survival of the fittest", "a new male identity", and "seeing other men in the world". After the diagnosis of prostate cancer, the body was viewed as being different. Some men talked about being confused that they survived the cancer despite being left impotent. Other men perceived that their bodies were less valuable in a social 
and personal sense because they could not conform to the conventional standards of masculinity. Sexual dysfunction and urinary incontinence were considered to be the major concerns of these men. The diagnosis of prostate cancer was perceived to be unpleasant and embarrassing due to the respective diagnostic procedures such as digital rectal examination and prostate biopsy. During confirmation of the diagnosis, men observed the cancer to be an alien entity in their body that was resented because of the physical, social, and emotional dilemmas that went along with it. During the interviews, men emphasized previous masculine identities as coping strategies. The professionals acknowledged the changes in men's bodies and the resultant consequences such as losing their social status in addition to their health. Men viewed their battle with cancer in terms of "losing the game of life" but recognized that they were at an advantage in comparison to others who lost their lives to cancer. Consequently, they perceived that they had a new male identity with a new body with limitations that was not able to function as it used to. The changed male ideology resulted from the perceived shift in the masculine role experienced as a result of their inability to engage in sexual activity. Due to their inability to engage in sexual activity, men felt that they needed to renegotiate their masculinity (Kelly, 2009). The study was conducted in Pakistan and yielded findings that are specific to a specific culture and area; nevertheless, the discussions are in concordance with studies conducted in other cultures (Oliffe, 2005). Men who could not live up to expected masculine roles in society renegotiated their masculinity to be able to come up with modified norms that would maintain their sense of being a man.

In a study with 95 elder U.S. veterans, Stansburry, Mathew-Chapman, and Grant (2003) studied the concepts of manhood and compared men diagnosed with prostate 
cancer with other military veterans at a Veterans Affairs medical center. An unmatched case-control design with purposeful sampling was used. Men diagnosed with prostate cancer who underwent either surgery or radiation therapy were recruited. The control group included patients at the urology clinic who complained of conditions other than cancer. The majority of the sample was White non-Hispanic $(n=80,84.2 \%)$ and married $(n=66,69.5 \%)$, with an average age of 68.4 years $(S D=11.6)$. Demographic data were collected using a survey. Additional data were collected using techniques adapted from cognitive anthropology (i.e., freelists, single pilesorts, and a three-point scale for assessing different attributes of masculinity). The men in the study highlighted the elements of masculine schema to include civic roles and masculine roles (i.e., boss, breadwinner, mentor, protector, citizen, head of house, provider). The term sexual was found to be closer to the main cluster of roles and values than to the physical attributes. Sexuality was perceived as an individual characteristic that is related to the moral roles and ideals in addition to the masculine identity. Findings showed that men perceived masculinity in terms of moral terms (i.e., integrity, responsibility, trust, helpfulness, dependability, and honor). Although sexuality was not central to the structure of masculinity in this sample, sexual identity and performance seemed to retain centrality in the veteran's roles. Findings documented that men with prostate cancer had a strong moral discourse about the meanings of being a man. Despite addressing a population with stronger sense of masculinity and powerful beliefs, the results allude to the importance of sexual potency for the masculine image of these men (Stansburry et al., 2003). 
In a qualitative study, Grunfed, Drudge-Coates, Rixon, Eaton, and Cooper (2013) explored the meaning of work among prostate cancer survivors and described the linkages between masculinity and work following prostate cancer treatment. Fifty participants who were between the ages of 18 and 65 years old, had a paid job prior to the diagnosis of prostate cancer, had no evidence of metastatic disease, and spoke English were recruited for the study from outpatient cancer departments in the United Kingdom. The participants were recruited to participate in the study within 4 weeks of treatment completion. Forty one participants were contacted again at 12 months after the completion of the treatment. The participants were 59 years $(S D=5.81)$, White $(n=38$, $76 \%)$, and underwent different types of treatment such as surgery $(n=33,66 \%)$, brachytherapy $(n=14,28 \%)$, and radiotherapy $(n=3,6 \%)$. Semistructured interviews were conducted with the consenting participants. Data were analyzed using a framework analysis approach. The majority of men returned to work by 12 months after treatment. Most of the participants returned to the same company $(n=35,85 \%$, with no change in duties $(n=32,78 \%$, and worked the same number of hours as prediagnosis $(n=31$, 76\%). The findings suggested four themes: work and self identity, work related implications of treatment side effects, disclosure of cancer, and future as cancer survivor. Most of the participants' reported urinary incontinence to be a prevailing concern that hindered their experience of going back to work (e.g., frequent bathroom breaks, fear of leak, embarrassment). There was an expressed anxiety about the practical issues related to dealing with incontinence while maintaining their normal work routines within a disclosed social context. The concepts of masculinity were identified throughout the transcripts of the interviews through the emphasis on the importance of work and the 
significance of the social interaction within the work environment. Men tried to hide physical hindrances to appear strong and capable. These findings show that after the treatment of prostate cancer, men may feel challenged by their social and work-related roles. This study highlights an important aspect of men's life after the treatment of prostate cancer. It focuses on the practicality of going back to work and its social and work implications on men. Additional research is needed to explore the experiences of men in different cultures with variety of labor laws and systems in addition to cultural norms emphasizing the breadwinner role of men (Grunfed et al., 2013).

In a study that linked masculinity to health outcomes, Burns and Mahalik (2006) examined the moderating effects of physical health and scripts for masculinity (i.e., self reliance and emotional control) on the relationship between powerful other people health locus of control (i.e., the power and influence of other people on the progression of their disease) and mental health in men treated for prostate cancer. Two hundred thirty men were recruited for the study. The Powerful Other People Health Locus of Control scale measured the men's perceptions that family, friends, and peers are important in controlling the progression of prostate cancer. The Physical Health Quality of Life Summary scale (i.e., physical functioning, role limitations, general health perception), mental health subscale of the SF-36 Quality of Life survey, and Conformity to Masculine Norms Inventory (self reliance and emotional control scales) were also used for data collection. Results suggested that men with poor physical health had poor mental health when having higher self reliance (i.e., masculine norm) and believed powerful others were important in fighting prostate cancer. The beliefs of men with poor physical health and strong powerful showed enhanced mental health outcomes when they had lower self 
reliance. Poor physical health contributed to poor mental health in men with strong emotional control (i.e., masculine norm) and strong belief in powerful other people. Men with poor physical health and strong powerful other people had positive mental health outcomes with strong emotional control. Findings from this study documented that physical health and masculine scripts (i.e., self reliance and emotional control) served as moderating variables in the relationship between powerful other people locus of control and mental health (Burns \& Mahalik, 2006).

In a study that examined the moderating role of sexual functioning on the relationship between men's enactment of traditional norms of masculinity and their social, role, and mental health after the treatment of prostate cancer, 234 men comprised the sample. The SF-36 Quality of Life Questionnaire was used to measure the men's social, role, and mental health functioning. The Expanded Prostate Cancer Index Composite (EPIC) was used to measure sexual and urinary functioning. The Conformity to Masculine Norms Inventory was used to measure masculine norms. Results showed that poor sexual functioning contributed to poor social functioning in men who endorsed traditional masculine norms. Men also reported that that with poor sexual functioning, they had poor role functioning when they conformed to traditional masculine norms. Poor sexual functioning led to poor mental health in men with strong masculine norms. Results from this study documented the mediating role that sexual functioning plays in the relationship between masculine norms and men's social, role, and mental health functioning (Burns \& Mahalik, 2008).

Hoyt, Stain, Irwin, and Thomas (2013) conducted a longitudinal study and used a cancer specific assessment to determine whether or not cancer related threat to 
masculinity predicts prostate related (i.e., urinary, bowel, sexual) functioning over time and whether or not cancer related emotional coping processes explain these relationships. Participants who had undergone radical prostatectomy or radiation therapy 2 years prior to the initiation of the study were recruited and interviewed at time of participation (T1), and after 2 (T2), and 4 (T3) months. Sixty six men with a mean age of 65.76 years $(S D=$ 9.04), White $(86 \%, n=66)$ and married or partnered $(n=66,89.4 \%)$ participated in the study. Data were collected using a cancer-related masculine threat instrument that was developed by the researchers, UCLA Prostate Index to measure prostate-specific functioning, Emotional Approach Coping, Emotional Self Efficacy Scale-Cancer, and Emotional Suppression Scale of the Emotion Regulation Questionnaire. Path analysis was used to assess whether or not emotional approach coping processes explained the contributions of cancer-related threat to masculinity in changing prostate-specific functioning. Controlling for baseline functioning and age, cancer-related masculine threat explained decline in (T1 to T3) urinary, bowel, and sexual function. Cancerrelated masculine threat also explained decreased emotional processing ( $\mathrm{T} 1$ to $\mathrm{T} 2$ ) but did not explain emotional expression. Decreased emotional processing explained the effect of cancer-related masculine threat on bowel and sexual function. It also predicted the decreased prostate-specific functioning. The findings from this study show that masculinity plays an integral role in prostate cancer functioning. When men believe that their cancer is inconsistent with their masculinity, they are at risk of decreased prostatespecific functioning after treatment. Cancer-related masculine threat significantly contributes to the coping responses of men and is negatively related to efficacy of emotion-directed coping. These findings suggest that having unthreatened masculine 
identity enhances the functioning after the treatment of prostate cancer (Hoyt et al., 2013). This study emphasized the importance of masculine roles and beliefs of gender roles on functioning after undergoing treatment of prostate cancer.

In summary, having a profound social and cultural influence, masculinity is also influenced by several sociocultural constructs. Boys have been socialized into emphasizing the concepts of sexual potency, superiority, and authority (Connell, 1995; Kimmel, 2000). Most of these constructs in the case of prostate cancer lead to the notions of the loss of authority and/or power which may influence the hegemonic masculinity of men (Connell, 1995). Wall and Kristjanson (2005) emphasized the psychosocial impact of prostate cancer in light of the concepts of hegemonic masculinities. Men were found to feel subordinated and less manly as a result of sexual dysfunction (Gray et al., 2002; Hawkins et al., 2009). Studies that have attempted to reframe the common masculinity norms to be able to embrace some conditions such as prostate cancer have highlighted several themes including health seeking behaviors, relationship issues, and sexual issues (Wall \& Kristjanson, 2005).

As a result of gendered societies, men enduring prostate cancer treatment side effects tend to rely on themselves and, therefore, will not ask for help or seek medical guidance (Burns \& Mahalik, 2007). Men's self reliance in health issues was observed to be a theme among all men, especially those diagnosed with prostate cancer. Men seeking medical attention were found to be perceived as less masculine or "macho". Accordingly, medical attention was sought in advanced cases (O'Brien et al., 2005). Decreased medical attention was also heightened by the fact that most urologists are men who are caring for men with prostate cancer; therefore, many psychosocial concerns may not have 
been discussed since the emphasis is based on the biophysiological symptoms and outcomes of the treatment (Oliffe \& Thorne, 2007). Men also try to exhibit self control and strong emotions as a result of their masculine expected behavior. Feelings of desperation, humiliation, and despair are reported by men who cannot initiate sex and thus feel less masculine (Oliffe \& Thorne, 2007). These feelings are important considerations for nurses and healthcare providers to be able to appropriately support and care for men with prostate cancer.

Men emphasized their role and image in society. Qualitative studies with men diagnosed with prostate cancer have discussed the importance of being able to control bodily functions in social settings (Chapple \& Ziebland, 2002). As a result, sexual dysfunction and urinary incontinence were a source of embarrassment, shame, and stigma (Chapple \& Ziebland, 2002). The decreased sexual ability was a major concern for men after diagnosis of prostate cancer due to the perceived importance of sexuality for the socially constructed masculine norms (Burns \& Mahalik, 2006; Chapple \& Ziebland, 2002; Fergus et al., 2002). Also, social norms of sexuality were affected by the age group of men and how they expressed the impact of sexual dysfunction on their lives (Oliffe, 2005). Younger men expressed their concerns about sexuality more openly than older men (Oliffe, 2005). The consequences of sexual dysfunction and urinary incontinence in light of the socially constructed masculine norms were found to result om decreased mental health functioning of men with prostate cancer, especially in those who emphasized strong and rigid masculine norms (Burns \& Mahalik, 2006).

Other sources that accentuate the importance of sexual performance and strength include the advertisement of products and media. The media continuously advertises the 
easy availability and accessibility of male enhancement gadgets and regimens. These messages emphasize the social expectation of men to be able to sexually perform, especially since the lead faces of these campaigns are athletes or socially prominent figures. Male sexual enhancement products that improve the sexual performance of men are elaborated in a manner that promotes charisma, charm, muscle, and satisfactoriness (Clarke, 1999; Stibbe, 2004). Therefore, the media supports the masculine image as described by Connell (1995). Masculinity has also been highlighted by the emerging studies that emphasize the importance of testosterone ("T"), the sex hormone, on levels on sexual activity (Loizides, Swierzewski, O’Neill, Griesser, \& Smith, 2004; Mazur \& Booth, 1998).

Irritable male syndrome and low $\mathrm{T}$ are concepts that are being recently discussed as concerns facing men during their later adult years. Consequently, these advertisements are providing a medical reason or cause for men who do not meet masculine concepts at older ages when testosterone levels tend to decrease (Loizides et al., 2004; Mazur \& Booth, 1998). Accordingly, men may perceive themselves as less masculine and, therefore, seek medical advice such as sexual male enhancements. This is especially true because most men in their older adult years still feel sexual (DeLamater \& Moorman, 2007) despite their diminished sexual abilities due to comorbidities such as decreased testosterone levels or prostate cancer. These factors may explain men's hesitancy to discuss their prostate cancer side effects (Fergus et al., 2002) as an attempt to save their masculine image. The medicalization of decreased sexual abilities may also provide the grounds to revert to prescriptions that would decrease their challenged sexual functioning. 
In summary, the diagnosis and treatment of prostate cancer results om challenges to men's masculinity. With socially expected sexually potent men, prostate cancer treatment side effects contribute to a perceived sense of embarrassment and shame. Men's decreased help seeking attitudes inhibit them from seeking appropriate help to manage better their sexuality. As a result, the QOL in these men is affected. However, the majority of studies that have examined the impact of prostate cancer on QOL have used qualitative designs with small samples of men that is difficult to generalize and only results of one quantitative study was published in two manuscripts. There remains to be a gap in the literature regarding the contributions of masculinity on QOL in men with prostate cancer using quantitative research designs.

\section{Relationship with Partner}

Sexual dysfunction as a result of prostate cancer treatment leads to emotional distress resulting in a series of responses including emotional distance between couples. Other reports on psychological effects on men with prostate cancer include isolation, anxiety, and depression (Hawkins et al., 2009). Studies that have included partners of men with prostate cancer have described their experiences in terms of decreased intimacy, emotional burden similar to that of caregivers, and a change of role. Women reported that they were no longer the lovers in the relationship, and romance was replaced by the resultant stress from the sexual issues (Badr \& Carmack Taylor, 2009; Giarelli et al., 2003). Additionally, resultant psychological and physical distress were reported to be major burdens on intimate relationships (Gilbert, Ussher, \& Hawkins, 2009). 
Erectile dysfunction was found to be significantly correlated with lower sexual and relationship satisfaction. McCabe and Matic (2008) found that in couples facing sexual dysfunction issues, there was increased conflict as well as decreased communication and sexual activity. Approximately $86 \%$ of men and $52 \%$ of women reported that sex was very or extremely important in a relationship. Contrary to men, female partners reported that erection problems were not imperative compared to other issues in their life $(t=-4.25, p<.001)$. Age was not included as a variable in this study to help understand if findings are applicable to all age groups. Also, decreased levels of intimacy predicted a decline in the overall relationship satisfaction (McCabe \& Matic, 2008). Badr and Carmack Taylor (2009) reported that erectile function and intercourse were positively correlated with marital adjustment and negatively associated with partner distress. In other words, despite controversy, it can be concluded that sexuality is an important contributor to relationship satisfaction and wellbeing; thus, sexual dysfunction as a result of prostate cancer may have a negative effect on partner relationship.

Couples with prostate cancer reported stressful relationships and distress that were affected by avoidant coping behaviors (Lavery \& Clarke, 1999; Wootten et al., 2007). Intimacy and communication were the major signs of relationship disruption. Promoting communication and education about intimacy issues were found to be essential to enhancing QOL in these couples (Galbraith \& Crighton, 2008). Men and women reported a major change in their roles whereby women assumed the role of caregivers and became less feminine and sexual. On the other hand, men felt that they could carry on the tasks they used to perform (Sanders et al., 2006). As a result, theses change created 
increased levels of stress that resulted in challenges in the relationship between the couples.

In a qualitative study that examined sexuality and intimacy of couples after the diagnosis of cancer, Hawkins and colleagues (2009) interviewed 156 partners and 20 patients with cancer. Approximately $76 \%$ of partners of patients with nonreproductive cancers and $84 \%$ of partners of patients with reproductive cancer reported an impact on their sexual relationship. More than half of the women (59\%) and more than three fourths of the men (79\%) reported decreased frequency of sex and intimacy. Renegotiation of sexual intimacy was a significant finding discussed by $19 \%$ of the women and $14 \%$ of the men. Findings from this study suggested two main themes: decreased frequency of sex and renegotiation of sex or intimacy. These themes were discussed in terms of dysfunction in which alternative intimacy behaviors were considered at first but then faded with time. Redefining of the role of partners as caregivers affected the sexual attractiveness between the couple and ultimately eliminated the image of the "lover". As a consequence of the cancer diagnosis, sexual experiences were altered resulting in intimacy and relationship issues. The changes in sexuality were linked to reports of self blame, rejection, sadness, and decreased sexual fulfillness (Hawkins at al., 2009). Findings from this study supported the premise that the acknowledgement of the changes in sexual relationships of couples with cancer is an important area to promote relationship satisfaction.

Galbraith and colleagues (2008) described health related outcomes of couples experiencing prostate cancer before treatment and at 6,12 , and 18 months. Men and their female partners ( $n=216$ couples) were enrolled in the study. Men and their female 
partners were provided with separate questionnaire packages and instructed to fill the questionnaires separately. Men in this study were on average 67.8 years ( $S D$ not reported) and married for an average of 34.8 years ( $S D$ not reported). The partners had a mean age of 64 years ( $S D$ not reported). The majority of the sample (i.e., men and their partners) was Caucasian (86\%). Men were treated by watchful waiting $(n=2)$, surgery $(n=39)$, conventional radiation $(n=8)$, mixed beam radiation $(n=48)$, and proton beam radiation $(n=109)$. Before the initiation of treatment, men scored significantly higher than their partners on relationship satisfaction, physical role function, emotional role function, mental health, and pain. Female partners had significantly better health than men at most of the time points. At 6 months after initiation of treatment, partners had better health-related QOL than men; on the other hand, men had better mental health functioning and general health. At 12 months, men continued to have better mental health, and the partners scored higher on health-related QOL and general health. Similar findings were reported at 18 months. Upon analysis of the overall summary of the scores, there was a significant difference in mental health scores during the pretreatment phase between men and their partners. Before treatment, men's scores significantly predicted partners' scores on relationship satisfaction, emotional role function, energy, mental health, and social function. At 6 months, men's scores predicted their partners' scores on health-related QOL, physical role function, emotional role function, mental health, and general health. At 12 months, a similar pattern was found in addition to partner's energy levels and social functioning scores. At 18 months, all variables were significantly different (i.e., relationship satisfaction, physical role function, emotional role function, and mental health) with the exception of bodily pain and general health 
between men and their partner. The partner relationship scores of men and their partners, measured by the Dyadic Assessment Scale (DAS), were significantly positively correlated at all the time points. Cross-lag analyses were conducted and showed that couples' scores had a reciprocal pattern of influence between the dyads at all time points. The men and their partners' mental health component scores were correlated before treatment. The partners' mental health component scores at 6 months predicted the man's scores at 12 and 18 months. The men's mental health scores at 12 months predicted the partners' scores at 18 months. Men's physical component scores before treatment predicted their partners' scores at baseline and 6 months. Similarly, partners' physical component scores at baseline predicted the men's scores at baseline and at 6 months. Men's scores at 6 months predicted their partner's scores at 12 months, a relationship mirrored by the partner's scores. Findings from this study suggested that the diagnosis and treatment of prostate cancer affect men and their partnerz (Galbraith et al., 2008). This study highlighted the effect of prostate cancer and treatment side effects on couples. However, a study limitation was that the sample was predominantly Caucasian, and men underwent different treatments. Also, the attrition rate was $26 \%$, and there was no description provided about the couples that dropped out.

Boehmer and Clark (2001) explored men's and their wives' perceptions upon prostate cancer diagnosis and their role in treatment decision making. In a sample of men diagnosed with metastatic prostate cancer and their wives ( $n=7$ couples), separate focus group interviews were conducted. Men in this sample were 61 to 75 years old. Five couples were White and two were African American. The couples were separately asked about the prostate cancer experience. Men discussed issues and problems faced prior to 
the diagnosis of prostate cancer (i.e., urinary and erection problems). The discussions included details of the symptoms and the process of seeking help. In the men's focus groups, few mentioned their wife. Men did not talk about discussing their problems or symptoms with their wives. Most of the men delayed seeking medical attention until the symptoms became severe or when they were admitted to a hospital for another disease and subsequently diagnosed with the cancer. During the focus groups with the wives, the interviewer posed the same questions. Women talked about how they knew about their husband's diagnosis. The difference in the first section of the focus groups was about the focus of the discussions. Men focused on their prediagnosis symptom,s and the wives stressed on the process of being informed about the diagnosis of prostate cancer. When discussing the treatment decision making phase, men and women talked about how the treatment was selected. Some men made the decision with their physician and totally excluded the wife. The wives of these men talked about not being informed about the diagnosis, treatment, and prognosis. Two of the men included the wife in every step of the decision making. This inclusion of the wife was suggested and emphasized by the physician and not the men themselves. However, this was not a concept discussed by the majority of men in this study. Men in this study did not make an effort to discuss their diagnosis with their wives. The inclusion of the wife was solely planned and carried by the physician. Two couples talked about a spousal partnership. They discussed the details of the diagnosis and treatment in which the physician was a third party. Findings from this study showed the different scenarios or roles that the partners of men with prostate cancer experience (Boehmer \& Clark, 2001). 
Gray and colleagues (2000) explored the issues of support and coping in couples diagnosed with prostate cancer. In a longitudinal qualitative study, the authors investigated the experiences of couples with the diagnosis and surgical treatment of prostate cancer. Thirty four men and their partners were interviewed before the surgery, 8 to 10 weeks post surgery, and 1 year post surgery. The interviews were conducted with men alone, women alone, and men and women together. Men were 50 to 68 years old, and the partners were 42 to 72 years old. A grounded theory approach was used to collect and analyze the data. Managing the impact of illness was the core category of the couples' experience. Five domains were identified: dealing with practicalities, stopping illness from interfering with everyday life, keeping relationships working, managing feelings, and making sense of it all. The couples in this study talked about being actively involved in every step from the diagnosis to treatment. These couples discussed several challenges but exhibited means to manage the impact of the cancer on their lives. The challenges started from the moment of diagnosis, but with time the couples became more informed about the disease and treatment. Proper communication and information about the disease helped couples deal with the illness. It was evident that the couples in this sample focused on continuing their lives rather than focusing on the presence of cancer and corresponding losses. Men and their partners tried to minimize the impact of prostate cancer during the discussions and focused on strategies to deal with the crisis. Men expressed less distress than their partners. Few men disclosed emotional struggles. On the other hand, men talked about relying on their partners for help, encouragement, and companionship. Nevertheless, the couples discussed challenges in the relationship. Many times, men would distance themselves from the partner while trying to act tough 
and in control in an attempt to meet their perceived manly expectations. The wives perceived their reactions as being hard on themselves while focusing on recovery. Women voiced their preference to have more communication with their husbands. However, men did not feel comfortable in exhibiting a need for their partner, while at the same time the women were reported to be their source of social support. In response, women were providing support without appearing to do so, and they were reassuring their husbands about their decreased potency. The requirements from women denied them the opportunity to use their own coping strategies and therefore they were more distressed than men (Gray et al., 2000). Findings from this study shed light on the experiences of couples diagnosed and treated for prostate cancer. However, the struggle of these couples cannot be generalized due to the qualitative nature of the study and the homogeneity of ethnicity of the sample. These findings, contrary to the previously discussed study (Boehmer \& Clark, 2001), suggested that the wives played a vital role by being a source of social support for men with prostate cancer. Men in this study also included their female partners in the discussions about their illness and tried to include them when finding ways to deal with the crisis.

In a similar study, Harden and colleagues (2002) explored the experience of couples living with prostate cancer, the impact if the illness on their QOL, and their ability to manage symptoms. Six focus groups were conducted: two with men only ( $n=$ 14), two with partner-caregiver only $(n=12)$, and two with dyads ( $n=8$ couples). The majority of men were Caucasian (64\%) and married (86\%). The focus groups yielded four themes: enduring uncertainty, living with treatment effects, coping with changes, and needing help. With the diagnosis of prostate cancer, men struggled with treatment 
options and were disturbed with the emerging interruption of their lives and roles. There were changes in the men's social lives and household responsibilities. After the treatment, men discussed losing urinary control, sexual difficulties, hormonal alterations, and overwhelming fatigue. As a result, they had to modify their lives to manage these challenges. Men discussed planning their schedules around the availability of restrooms. Many men grieved the loss of their sexual functioning and the challenges of that faced their marriage. Women acknowledged the distress with sexual dysfunction but were willing to work with it because they did not want to lose their husbands. Men treated with hormonal therapy experienced mood swings, hot flashes, and weight gain. The couples were not prepared for these symptoms and therefore were in deep distress. The challenge of the masculine image of the man was discussed by the group undergoing hormonal treatment. The changes experienced by couples contributed to a new family lives. Some couples talked about being together more often and not being able to engage in outside social events. Couples also had some shifting roles in which the men and their female partners could not accomplish some of the tasks they used to perform. However, the couples still mentioned that they tried to manage the situation but needed help in dealing with their situations. Help was sought from professional resources and individualized information that would work in their cases. Findings from this study indicate that couples go through turmoil of emotions and challenges. The need for support and more information was highlighted throughout the study (Harden et al., 2002).

Fergus and colleagues (2002) explored the patient-provided support for spouse caregivers within the context of prostate cancer. Thirty four couples were interviewed before surgery, 2 months after surgery, and 1 year following surgery. Men who 
participated in this study were 50 to 68 years old and married between 12 to 41 years. The women were 42 to 72 years old. The semistructured interviews included questions about supportive interactions between partners. Findings yielded four domains of patient-provided support for their spouse caregivers: easing spousal burden, keeping us up, considering spouse, and maintaining connection. Men were conscious about the burden that their disease and their side effects have on their partners. Men tried to decrease the burden by taking care of themselves and doing things on their own. Some men thought that keeping their wives away would alleviate the burden on them. Men also took a positive standpoint concerning the cancer so as not to distress their partners. They tried to control their mood and disappointments. Wives discussed trying to get closer to their husbands to spend more time with them and provide support. As a result, the couples were spending more time together. Men encouraged their partners to be more independent and take care of themselves. Wives perceived the new relationship to be more caring, although men focused on the distress of erectile dysfunction. Men talked about prostate cancer to be a disease of the whole family that led them to spend more time together, share experience, and express affection. Findings from this study shed light on the role of men diagnosed with prostate cancer and how they tried to take control of their partner's social and emotional wellbeing (Fergus et al., 2002).

Giarelli and colleagues (2003) described wives' self reports of preparedness to care for their husbands' physical and emotional needs during the first 6 months after prostatectomy. The mixed methods clinical trial had two groups: the intervention $(n=$ $57)$ and control groups $(n=53)$. The intervention group received standardized nursing intervention protocol that included information provided by an advanced practice nurse 
about symptom management, restoration of urinary incontinence, and promotion of marital communication and sexual intimacy. The participants were 32 to 73 years old and predominantly Caucasian. Data were collected using the Preparedness for Caregiving Scale (PCGS) and an open ended question at baseline and 3 and 6 months following prostatectomy. Results were presented in two sections: quantitative and qualitative findings. The PCGS showed no difference between the intervention and control groups in reporting preparedness to take care of their husbands' physical and emotional needs. Preparedness scores were found to increase over time in both the intervention and control groups. However, at 3 months, preparedness scores of the wives in the control group slightly decreased. During the same time, the wives in the intervention group scored higher in preparedness. The increased preparedness of wives was thought to be the effect of the advanced practice nursing intervention. Qualitative findings showed three main themes: prepared with practical information, prepared for emotional responses, and prepared with resources for problem solving. Wives described their emotional turmoil with the care of their husbands and requested practical information to deal with physical and emotional needs. Emotional needs were not only for the men but also for the wives who reported to have similar distress levels. At 3 months, the practical information was needed to deal with side effects such as incontinence. There was a need in both groups for information about sexual dysfunction and ways to deal with the emotional needs and maintenance of effective communication. At 6 months, women in the intervention group did not require practical information; however, men in the control group still asked for explanations about physical needs. Feelings of fear, apprehension, impatience, distress, and unwillingness to care were 
experienced by both wives and husbands. Wives wanted to be prepared for their new role as a caregiver with the loss of support of their husband and the emotional distress. At 3 months, both groups still reported emotional distress. However, the control group still needed to be prepared for emotional needs and distress. The intervention group needed ways to deal with depression, discouragement, exhaustion, and distress associated with the role changes and the memories or better times. At 6 months, there was a difference in the preparedness between the groups. Wives in the intervention group discussed the need to deal with husbands' anxiety and depression. On the other hand, wives in the control group discussed feelings of uncertainty that were transformed into frustration and more distress. Preparedness for problem solving included being able to identify resources for social support, spirituality, time management, and identifying personal strengths and weaknesses. At 3 months, the resources for problem solving included social support, self awareness, and marital relationship. Nurses were identified as being important sources of social support by both groups. Similar findings were reported at 6 months. Findings from this study documented the importance of including families, especially wives, in the treatment path of men with prostate cancer. Wives are the source of social support, and they are equally affected by the diagnosis (Giarelli et al., 2003).

Harden and colleagues (2006) examined the experiences of men with prostate cancer and their partners according to their life cycle cohort: 50 to 64 years (i.e., late middle age), 65 to 74 (i.e., young old), and 75 to 84 (i.e., old old). This qualitative study aimed to compare the age-related differences in couples with a man who was diagnosed and treated for prostate cancer. Five couples per group were recruited using purposeful sampling. The majority were Caucasian ( $n=14$ couples), and all partners were women. 
Analysis of the semistructured interviews extracted three themes: couples' daily lives, their dyadic and family relationships, and developmental issues. The majority of men in the late middle age group talked about being distressed and stressed about not being able to fulfill their perceived role in the family. These men reported being depressed or being treated for depression. One wife talked about having to retire early to be able to be the caregiver of her husband, and one man talked about being forced to take early retirement to be able to deal with disease side effects. The daily lives of couples was summarized by feelings of fatigue, changing priorities, and financial concerns. When talking about dyadic and family relationships, intimacy issues and talks about being "in it together" emerged. Couples in the middle aged group compared themselves to friends who had other chronic and fatal diseases. Couples with prostate cancer reported they were struggling with cancer and sexual issues. They preferred that the diagnosis of prostate cancer was given to them at a later stage in life. The young old group of couples had a more positive perspective. Their lives were not as disrupted by the cancer. Intimacy issues were related to impotence as a result of prostate cancer treatment. The wives discussed the change in sexual patterns in their relationship as affecting their overall lifestyles. Couples in this group also compared themselves to others their age and, therefore, accepted the positive prognosis of their disease. They accepted the timing of the diagnosis. The oldest group reported that the diagnosis of prostate cancer interrupted their plans and daily lives. Dyadic and family relationships revolved around intimacy issues and togetherness. Men felt uncomfortable with not being able to continue with their sex lives, which was a source of distress. The discussions of the couples reflected the togetherness of the relationship. They seemed to do many things together. When 
comparing themselves with others in their age group, the diagnosis of prostate cancer did not seem to be as bad as they thought due to its positive prognosis. However, the men talked about having limited treatment options as a result of their age and other comorbidities. Findings from this study suggested that the lives of couples are affected by the diagnosis and treatment of prostate cancer irrespective of age. However, priorities and the manner that the disease affected the couples and the degree of positivism were different between the age groups. Couples were interviewed together, and there may be a possibility that men and women may not have had the courage or opportunity to openly express themselves.

Sanders et al. (2006) conducted a qualitative study to investigate the concern of couples who were surviving prostate cancer and treatment. Three 30-minute segment focus groups were conducted with 10 couples to discuss their experiences as a couple, as individual men and women, and regarding intimacy issues and relationship needs. Men and women in the first focus group were together; then they were separated in the second, and they were brought back together in the third. Men were 45 to 81 years old, and the women were 38 to 75 years old. Findings suggested that the men's and women's experiences with the diagnosis of prostate cancer were different. Women saw that their role evolved from being the wife and lover that was being transformed into being the caregiver. Sexual relationships were significantly different and led wives to feel unattractive and less as a woman. Sexual dysfunction led to a decrease in romance and intimacy which were reported by women to be a source of frustration. Men, on the other hand, felt castrated as a result of erectile dysfunction and urinary incontinence. There was a feeling of fatigue that prohibited men from sharing in chores and duties around the 
house. Men talked about major communication issues with their wives. When grouped together, the couples agreed that they needed more information and guidance to manage as a couple. The preferred mode of information was different between men and women. Women wanted to participate in support groups to be able to talk to other couples who were going through the same experience. Men, on the other hand, preferred receiving brochures and written material. The couples agreed that regardless of the source, information received needs to be individualized (Sanders et al., 2006). Findings from this study showed the experiences of couples with prostate cancer that changed the romantic role of the women to a caregiver image. The relationship in couples was affected by the diagnosis and treatment side effects as reported by the participants in the study. The study also showed that the couples needed more information about the course of the disease and what to expect. However, husbands and their wives had different preferences regarding the delivery of desired information.

Hawes and colleagues (2006) described the problems chosen as the focus of problem-solving therapy by spouses and men with prostate cancer. This study was part of a randomized control trial that tested the therapeutic value of problem solving skills training for couples coping with prostate cancer. In a descriptive, cross-sectional study, 66 spouses and partners answered a survey that included the Problem-Solving-InventoryRevised (SPSI-R) and the Profile of Mood States (POMS). The majority of the couples was married $(85 \%)$ or cohabitating $(15 \%)$. Most of the sample was White $(82 \%)$ and age 65 years or older (37\%). The first two problems identified by couples were categorized to determine the major problems encountered. Four problem categories were identified: treatment and side effect issues, patient issues, family issues, and spouse issues. Couples 
wanted more information about the cancer, new treatment trends, and medications. Urinary incontinence and impotence were sources of frustration for the patient. Men were viewed as being sloppy and not taking care of themselves, and they engaged at times in risky behaviors (i.e., smoking, no nutritional eating habits). Men were also viewed as having a bad temper and at times were verbally abusive. Scheduling dinner and other social events was an issue. Relationship with the spouse and children living at home were also jeopardized. The spouses considered their emotional wellness to be a major concern. They did not perceive that they had time for themselves anymore which increased their anxiety levels, decreased their self esteem, unbalanced their temper, and caused complaints of a declined physical health conditions. Findings from this study highlighted major spousal issues (i.e., women's emotional wellness, balancing their medical concerns with their husbands' conditions) and patient issues (i.e., men's lack of communication, depression) (Hawes et al., 2006). Identifying the key concerns and problems of couples struggling with prostate cancer will help in recognizing the best suitable ways to plan appropriate healthcare plans.

Kershaw and colleagues (2008) sought to determine if baseline antecedent variables and intermediary appraisal variables predicted coping strategies and QOL outcomes. The majority of the sample in this study $(n=121,67 \%)$ was newly diagnosed with prostate cancer for an average of 8 months. Most of the sample was White (86\%) and married for an average of 31.8 years $(S D=14)$. Results of this study suggested that men with prostate cancer and their spouses scored higher on mental QOL and lower on physical QOL compared to the general population norms. Findings presented a longitudinal analysis of factors associated with QOL in prostate cancer patients and their 
spouses. Baseline variables (i.e., self efficacy, current concerns, age, socioeconomic status [SES], social support, communication, general symptoms, phase of the disease) were found to have effects on couples' appraisal at 4 months and on their coping and QOL outcomes at 8 months after the initiation of the study. Using the stress-coping model as a conceptual framework, the model accounted for $40 \%$ of the variance in patients' mental functioning and $34 \%$ of the physical QOL outcomes at 8 months. Men who had more active coping, lower avoidant coping, less hopelessness at 4 months, and fewer symptoms at baseline had improved mental health functioning at 8 months. Physical QOL was associated with less negative appraisal of illness at 4 months, fewer baseline symptoms, and new onset of the disease. The model accounted for $43 \%$ of the variance in spouses' mental functioning and $24 \%$ of the physical QOL outcomes at 8 months. Better mental health at 8 months was predicted by less active avoidant coping and less negative appraisal at 4 months and less current concerns at baseline. Spousal physical QOL was enhanced at 8 months if they were younger and had fewer baseline symptoms. The model accounted for $19 \%$ of the variance in the patients' active coping and $16 \%$ in avoidant coping at 8 months and was influenced by baseline social support and negative appraisal of illness at 4 months. The model accounted for $10 \%$ of the variance in spouses' active coping and $27 \%$ of avoidant coping at 8 months and was influenced by baseline patient symptoms, spouses' negative appraisal of illness, and patients' uncertainty at 4 months. Findings from this study supported the stress-coping theory and suggested how antecedent personal, resource, and illness variables at baseline influenced appraisal variables at 4 months. Also, strategies when utilized influenced QOL outcomes (Kershaw et al., 2008). Findings need to be considered with caution 
because structural equation modeling was used for analysis despite the small sample size. Also, the study was conducted without controlling for race/ethnicity or treatment modality which may have effects on QOL outcomes and course of coping and adjustment of couples.

Badr and Carmack Taylor (2009) examined the sexual functioning of prostate cancer patients and their partners and investigated the relationship between sexual dysfunction and psychosocial adjustment in relation to spousal communication patterns. One hundred sixteen couples answered surveys that included the International Index of Erectile Function (i.e., patient's sexual function), Female Sexual Function Index (i.e., partner's sexual function), DAS (i.e., marital adjustment), Centers for Epidemiological Studies Depression Scale (i.e., psychological distress), and Communication Patterns Questionnaire (i.e., spousal communication patterns). The majority of the sample was White (83.7\%), retired (54.5\%), and married (99.2\%). The average age of men in the sample was $67.4(S D=7.94)$. More than half of men $(52 \%)$ were undergoing treatment during the time of the study. Partners were also predominantly White (82.9\%), retired or unemployed $(66.9 \%)$, and had a mean age of 62.7 years $(S D=8.32)$. Findings from the study showed that patients and partners had high rates of sexual dysfunction. There was a strong positive association between the couples' sexual functioning and spousal communication patterns. Partners of patients with poor erectile function were likely to report decreased open spousal discussions which was associated with partners' marital distress ratings. Regardless of sexual functioning, couples with increased mutual constructive communication had better marital adjustment. Greater sexual dysfunction was associated with lower mutual constructive communication and poor marital 
adjustment in patients and their partners. Findings from this study suggest that healthy communication and sexual rehabilitation may enhance marital adjustment in couples struggling with prostate cancer (Badr \& Carmack Taylor, 2009).

Wu, Mohamed, Winkel, and Diefenbach (2013) explored whether spouses' illness beliefs about the timeline of illness mediated relations between spouses' illness beliefs about treatment control and patient's QOL 6 months after participation in the study. Fifty three men undergoing treatment for localized prostate cancer and their spouses participated in this study. Men were 64 years old $(S D=9.0)$ and their spouses were 60.6 years old $(S D=9.5)$. The majority of participants were Caucasian $(92.5 \%, n=49)$, and underwent radiation therapy $(52.8 \%, n=28)$, and surgery $(26.4 \%, n=14)$. Data for treatment control and timeline beliefs were collected from the patient and spouse using the treatment control and the timeline subscales of the revised Illness Perception Questionnaire. Data on patients' general QOL was collected using the Functional Assessment of Cancer Therapy-General instrument. Data on patients' urinary and sexual function were obtained using the American Urological Association symptom index and sexual adjustment questionnaire, respectively. The results suggested that spouses' timeline beliefs mediated the relationship between spouse treatment control beliefs and patient QOL. A similar relationship was not found when patients' beliefs were considered. These findings emphasize the importance of including spouses in discussions about the treatment and outcomes of prostate cancer. Spouses' beliefs about the positive treatment outcomes enhanced the QOL of patients (Wu et al., 2013).

In summary, the diagnosis and treatment of prostate cancer affects couples faced with the disease. These couples' relationships are influenced by the responses of men to 
their sexual dysfunction. Sexual dysfunction as a result of prostate cancer was reported to influence the psychological status of couples and generate feelings of social isolation, emotional distress, anxiety, and depression (Hawkins et al., 2009). Research has shown that there is a relationship between couples' sexual functioning, open communication, and relationship satisfaction (Badr \& Carmack Taylor, 2009). As a result, the relationships of couples had suffered from decreased intimacy and emotional burden (Badr and Carmack Taylor, 2009; Giarelli et al., 2003). The decreased intimacy levels led to a decline in the overall relationship satisfaction (McCabe \& Matic, 2008). Couples discussed the modifications in the image of the woman from the romantic partner to the female caregiver (Gilbert et al., 2009; Hawkins et al., 2009). As a result, the couples described their relationships as stressful and accompanied by avoidant behaviors (Wootten et al., 2007).

Intimacy issues were directly related to the satisfaction with the relationship (Galbraith \& Crighton, 2008; Hawkins et al., 2009). The diagnosis of prostate cancer did not affect men alone; it also affected female partners (Galbraith et al., 2008). However, male and female partners were found in one study to respond differently to the illness (Boehmer \& Clark, 2001). In this study, some men were found to alienate their wives from discussions about the disease, others included their wives because the physician insisted, and two couples formed a spousal coalition with the physician acting as a third party (Boehmer \& Clark, 2001). However, other studies have shown that female partners play a significant role in being the major source of social support (Gray et al., 2000). The focus of these couples was to manage the illness as a couple and find ways to maintain the relationship status (Gray et al., 2000). Fergus and colleagues (2002) suggested that 
men were trying to control their disappointments and mood changes to decrease the burden on their wives. Communication and adequate information about the course of the disease were identified to be key factors that helped couples deal with prostate cancer and its treatment side effects (Giarelli et al., 2003; Gray et al., 2000; Harden et al., 2002).

Other challenges that faced couples included the change in the men's social life (Harden et al., 2006). Harden and colleagues (2002) showed that men had to modify their priorities, household responsibilities, schedules, and other issues to accommodate the urinary incontinence and sexual dysfunction that accompanied the treatment of prostate cancer. These challenges were reported to bring couples together since they were spending more time together and less social engagement time (Fergus et al., 2002; Harden et al., 2002, 2006). Few studies examined the contribution of men's perceived relationships with their partners on men's QOL. Also, other variables such as age, race/ethnicity, and masculinity have not been examined in relation to the relationship with partner to understand their independent and combined contribution to QOL in men with prostate cancer.

\section{Age}

Sexuality and sexual functioning of adults (i.e., adults in their 40s, 50s and 60s) are controversial issues. People 65 years old and older are considered to be older adults. There were few studies that showcased the importance of sexual functioning of older men. The hesitancy of this discussion may be influenced by the stereotypes that older men engage less than younger men in sexual activities. The value of sexual functioning for men 50 years and older is important to explore in an attempt to understand the influence of sexual dysfunction in men diagnosed with prostate cancer. 
The National Social Life, Health, and Aging Project was conducted to report the prevalence of sexual activity, behaviors, and problems in a national probability sample of 3,005 U.S. adults (i.e., 1,550 women and 1,455 men) 57 to 85 years of age. Findings suggested that the majority of older adults (i.e., adults in their 60 s and older) had a partner and were involved in an intimate relationship. Sexuality was considered to be an essential part of their lives. However, the prevalence of sexual activity decreased with age (i.e., $73 \%$ in adults 57 to 64 years, $53 \%$ in adults 65 to 74 years, and $26 \%$ in adults 75 to 85 years). Men were more likely to engage in sexual activities despite $37 \%$ of men reporting erectile difficulties. Interestingly, both men and women with decreased sexual activity rated their health as being poor (Lindau et al., 2007).

Araujo, Mohr, and McKinlay (2004) described the individual changes in sexual function (i.e., sexual intercourse, erection frequency, sexual desire, ejaculation with masturbation, satisfaction with sex, and difficulty with orgasm) over 9 years in a population based cohort study. A sample of 1,085 men participated in the study. Findings showed that there were significant changes in all of the sexual function domains with the exception of the frequency of ejaculation with masturbation. The changes in individual sexual function over time were strongly associated with age. With increasing age, there was a significant decline in sexual function. The frequency of sexual activity decreased less than once per month, less than two times per month, and less than three times per month in men in their 40s, 50s, and 60s, respectively. Similarly, the number of erections decreased by 3,9 , and 13 in men in their $40 \mathrm{~s}, 50 \mathrm{~s}$, and $60 \mathrm{~s}$, respectively. Findings from this study showcase the developmental decline in sexual activity with age (Arauju et al., 2004). The reason for sexual decline was not explained by the researchers, 
but it may be due to the emergence of diseases that are common at the identified age cohort. However, the sexual desire and interest in sexual activity was not shown to be affected by age (Laumann, Glasser, Moreira, \& GSSAB [Global Study of Sexual Attitudes and Behaviors] Investigators' Group, 2009).

Age was found to be a significant predictor of prostate cancer. After the age of 40, the prostate cells tend to multiply causing hypertrophy of the gland. However, this increases the risk of developing cancerous cells (PCF, 2011). Trends have shown that there was a significant increase in prostate cancer diagnosis with increased age (ACS, 2011; PCF, 2011). The majority of men (97\%) diagnosed with prostate cancer were 50 years and older (ACS, 2011), and 65\% of men were 65 years and older (PCF, 2011). With the positive life expectancy associated with prostate cancer, these men were anticipated to live longer with the outcomes of their disease.

Studying the prognosis of prostate cancer, there were no significant differences by age at diagnosis. Examining the long term outcomes and 10 years life expectancy suggested that age was not a predictor of prostate cancer survival (Bill-Axelson et al., 2005; Wong et al., 2006). Bill-Axelson and colleagues (2005) reported the 10 years outcomes of two groups of men undergoing radical prostatectomy $(n=83)$ and watchful waiting $(n=106)$ as a treatment for prostate cancer. The percentage of death due to prostate cancer increased from $2 \%$ after 5 years to $5.3 \%$ after 10 years. There was a significant difference in deaths from prostate cancer between the two groups with more deaths in the surgery group. Findings suggest that surgery had better functional outcomes (i.e., sexual and urinary functioning) for men 65 years old and younger (Bill-Axelson et al., 2005). Similar findings were reported by Wong and colleagues (2006). Active 
treatment (e.g., surgery or radiation) in low and intermediate risk prostate cancer in men ages 65 to 80 years was found to have better 5 and 10 years survival rates (Wong et al., 2006).

Age was hypothesized to be associated with the outcomes of treatment (i.e., sexual dysfunction and urinary incontinence). Stanford and colleagues (2000) suggested that age was a significant contributor to sexual and urinary functioning after prostatectomy. Results were replicated in a sample of men undergoing nerve sparing prostatectomy (Penson et al., 2005). It was found that age contributed to urinary incontinence (Sacco et al., 2006) and sexual functioning (Rondorf-Klym \& Colling, 2003; Sanda et al., 2008). Harden and colleagues (2006) examined the experiences of men and their partners according to their life cycle. Findings suggested that all age groups had issues with their relationships, intimacy, changing priorities, distress, and other issues (Harden et al., 2006). Nevertheless, younger couples had more positive perspectives of their relationships, intimacy, changing priorities, and other issues (Harden at al., 2006). In a qualitative study, Oliffe (2005) suggested that older men were expected to place less emphasis on sexuality. As a result, they were hesitant to expand the discussion about the influence of decreased sexuality on their perceived masculinity. On the other hand, younger men were able to elaborate on the importance of their sexuality more openly (Oliffe, 2005).

In summary, age is an important variable to consider when studying men with prostate cancer. Age was reported to be a risk factor for the development of the disease (ACS, 2011; PCF, 2011). Men after the age of 50 years are at a higher risk for being diagnosed with prostate cancer (ACS, 2011; PCF, 2011). Research examining the 
outcomes and survival rates of prostate cancer has indicated that the 5- and 10-year survival rates were 100\% and 97\% respectively (Bill-Axelson et al., 2005). However, age was a factor in determining the functional outcomes of treatments (i.e., surgery, radiation, watchful waiting) (Bill-Axelson et al., 2005). Irrespective of age and treatment, men diagnosed with prostate cancer were at a high risk of developing sexual dysfunction (Rondorf-Klym \& Colling, 2003; Sanda et al., 2008) and urinary incontinence (Sacco et al., 2006).

On the other hand, the majority of adults in their 50s, 60s, and older had a partner and were involved in an intimate relationship. Sexuality and sexual activity were important to them despite the reported decreased rates with age. Men after the age of 50 are at a higher risk of developing erectile difficulties; however, they were found to be more likely to be interested in engaging in sexual activities. Sexual activity was an indicator of good health for men and women after the age of 50 years (Lindau et al., 2007). Age was also associated with decreased sexual activity, although sexual desire did not change (Laumann et al., 2009). Findings may be related to the emergence of medical conditions that may hinder sexual activities at later ages. As a result, men in their 50s and older who are at higher risk for developing prostate cancer (ACS, 2011) are subject to sexual dysfunction and may be disappointed when their sexuality is jeopardized. Consequently, due to the sexual dysfunction, men may face intimacy challenges with their wives and therefore their relationship satisfaction may be compromised (Harden et al., 2006). Problems related to sexual dysfunction are not often reported due to the social understanding that sexual activity is expected to decrease and be less emphasized with age (Oliffe, 2005). With sexuality being important for all age 
groups, sexual dysfunction is anticipated to affect men of all ages and consequently may influence their QOL.

\section{Ethnicity}

Ethnicity refers to people from a shared heritage and identity such as a common language, culture, or ideology. It can also be referenced as a shared belonging to a common geographic area (i.e., Hispanic) (Isajiw, 1974; Nagel, 1994). In research, race and ethnicity have been used as variables but not consistently defined by researchers (Drevdahl, Taylor, \& Phillips, 2001; Sheldon \& Parker, 1992). The majority of studies have used race/ethnicity as a single category and have included African American, Hispanic, and others in a single item. Ethnicity, in this proposal, will be able to capture the self-identified cultural affiliation (Bradby, 2003) in addition to the U.S. Census 2010 categorization. Additional categorization in the Hispanic group will be added to include the Hispanic descendants in the sample to be able to represent the uniqueness of the population in Miami, Florida (e.g., Cuban, Nicaraguan, Dominican, Colombian, and Venezuelan).

Studies identified racial disparities in the prevalence of prostate cancer. As previously discussed, it was reported that African American men have the highest incidence of prostate cancer followed by Hispanic men (Edwards et al., 2010). Nonetheless, few studies have examined the differences in outcomes (i.e., sexual dysfunction, urinary incontinence, masculinity, partner relationship, QOL) among men from different racial/ethnic backgrounds. Stanford et al. (2000) identified a difference in sexual functioning among ethnicities in which African American and Hispanic men reported better erection functioning compared to Caucasian men 18 months after radical 
prostatectomy. The details of the study were previously explained. The majority of studies among men diagnosed with prostate cancer were conducted among samples that self identified as non-Hispanic Whites or Caucasians. Some studies had 12 to $14 \%$ of their sample from different ethnic groups; however, they did not compare or report separate findings from these ethnicities.

Miami is a multiethnic and multiracial metropolitan area that is predominantly Hispanic. There is evidence that has suggested that cultural and ethnic constructions influence health behaviors of men (Addis \& Mahalik, 2003; Courtney, 2000). In a Hispanic community that emphasizes the concepts of machismo and caballerismo, the masculinity of men who experience sexual dysfunction may be challenged. Machismo is a socially constructed phenomenon that emphasizes the Hispanic male gender expectations and the meanings of being a Latino man (Arciniega et al., 2008). The traditional role of a Hispanic man has been identified in terms of being strong, a family protector, provider, and sexual prowness (Arciniega et al., 2008). The concept of machismo is closely related to the concepts of hypermasculinity and traditional masculine roles discussed by Connell (1995). The machismo beliefs are interrelated with strong and rigid traditional masculine gender roles (Abreu, Goodyear, Campos, \& Newcomb, 2000). Latino and Hispanic men who endorse their ethnicity and ethnic belonging were found to have greater adherence to traditional masculine ideologies and hypermasculine attitudes (Abreu et al., 2000). Men's genital organs are considered to be a sense of pride as well as symbols of their masculinity and being a man (Lugo, 1990). Consequently, any man who does not conform to this normal or expected understanding may be considered less of a man and may not be considered macho (Lugo, 1990). As a result, belief in machista may 
have an influence on health behaviors and health outcomes (Rivera-Ramoz \& Buki, 2011).

Maliski, Rivera, Connor, Lopez, and Litwin (2008) explored how African American and Latino men maintained masculine identity with prostate cancer treatment side effects. A total of 60 Latino and 35 African American men were interviewed using a semistructured guide. Transcripts were analyzed using a grounded theory technique and generated a descriptive model. Findings suggested that men's constructions of their masculinity were influenced by their experiences in their early lives. Prostate cancer treatment side effects and sexual dysfunction challenged their masculine identity. As a result, these men renegotiated their masculine identity to maintain their image and manly role in society (Maliski et al., 2008). The Latino men in this study were generally of Mexican origin; therefore, these findings may not be similar in a Miami sample that is predominantly Cuban and other Caribbean as well as Central and South American ethnicities.

Research with men with prostate cancer has generally not represented the experiences of men from minority ethnic groups. R. A. Jones and colleagues (2011) explored cancer support and financial issues related to prostate cancer in a sample of 23 rural and urban 65 years old and older African-American men. Using a descriptive qualitative design, five focus groups were conducted. Men were recruited from community-based centers (e.g., churches, barbershops, diners, and primary care clinics). The analysis of the focus groups yielded two main themes: family and physician support are important, and insurance is a necessity for appropriate health care. Female partners of African American men with prostate cancer were the main source of social support. 
These themes represented the main concerns of African American men with prostate cancer. There was a difference between rural and urban men. African American men living in rural areas expressed the importance of spirituality in helping them deal with the diagnosis and treatment of prostate. Insurance and financial issues related to healthcare treatment were also important to decreased QOL in both rural and urban men with prostate cancer (R. A. Jones et al., 2011).

In a sample of 17 African American men using a phenomenological approach, Jones, Steves, and Williams (2010) explored how and when they decided to go for prostate cancer screening. The majority of men discussed their limited education about prostate cancer and that they were not aware of the increased risk of African American men. Men emphasized the importance of having a family member involved in the decision to go for prostate cancer screening. The involvement of a family member was needed because they were accustomed to seeking health advice from informal sources such as family members (e.g., wife, daughter, sister). Men in this sample placed great emphasis on their trust in physicians. They discussed the importance of having their physicians insist that they perform prostate cancer screening. However, this trust was related to having a long standing relationship with the physician. Men who do not have a long standing relationship with a physician or healthcare provider did not agree with this concept (Jones et al., 2010). Findings from this study may suggest that family and health provider trust are important for African American men with prostate cancer. Therefore, their availability may contribute to QOL in African American men with prostate cancer.

Research studies that have used samples of Hispanic men with prostate cancer are scarce. In a randomized control trial, the short-term effects of a community-based 
intervention were conducted with Hispanic men aiming to encourage informed decision about prostate cancer screening with PSA. Senior social and housing centers were randomized into the intervention. The intervention group attended a Spanish language educational program facilitated by promotores (i.e., 12 centers; $161 \mathrm{men}$ ). The control group promotores attended diabetes awareness video and discussions (i.e., 13 centers; 160 men). Findings showed that $44 \%$ of participants had previous PSA testing at baseline. After the intervention, the intervention group reported knowledge gains, demonstrated increased awareness about PSA testing, and showed more involvement in decision making roles regarding testing. Men in the intervention group believed that it was important to consider the advantages and disadvantages of screening and anticipated that the decision to go for prostate cancer screening was going to be easier. The control group did not exhibit any changes in their intentions or beliefs about choosing whether or not to be screened for prostate cancer (Chan et al., 2011). Findings from this study support the importance of community based programs that increase awareness of prostate cancer screening using culturally and linguistically appropriate methods. Findings from this study also suggest that culturally appropriate awareness about the course of prostate cancer treatment may be beneficial for Hispanic men.

In summary, racial and ethnic backgrounds of men may contribute to the risk for developing prostate cancer (Edwards et al., 2010). African American men have the highest incidence of prostate cancer followed by Hispanic men (Edwards et al., 2010). Stanford and colleagues (2000) identified differences in sexual functioning among ethnic groups in which African American and Hispanic men reported better erection functioning compared to Caucasian men 18 months after radical prostatectomy. However, prostate 
cancer side effects (i.e., sexual dysfunction and urinary incontinence) and their influence on QOL in African American and Hispanic men have not been well reported in the literature.

With prostate cancer resulting in sexual dysfunction and urinary incontinence, concepts of masculinity and the impact of these side effects on QOL is in question. The Hispanic community's strong and rigid masculine constructions of machismo may influence these men's reactions to the disease. Research on the machista beliefs of Hispanic men and their influence on health behaviors and perceptions health outcomes suggest that Hispanic men may be distressed as a result of prostate cancer treatment side effects (Rivera-Ramoz, \& Buki, 2011). It can be anticipated that Hispanic men diagnosed with prostate cancer will have similar experiences to findings reported from studies with White males with strong and rigid conformity to masculine norms (Addis \& Mahalik, 2003; Courtney, 2000; Maliski et al., 2008). Similarly, it can be anticipated that as a result of prostate cancer treatment side effects, men's relationships with their female partners may be influenced. Consequently, men's QOL will be challenged.

The impact of sexual dysfunction on masculinity and impressions of African American men with prostate cancer has not been extensively examined in the literature. However, research with African American men has addressed prostate cancer screening attitudes and beliefs. Research may have focused on this area due to the increased risk of developing prostate cancer in African American men and the importance of early screening (Edwards et al., 2010). Studies that have examined the attitudes and beliefs about prostate cancer of African American men highlight the importance of social support of the wife and family in addition to the influence of healthcare providers in 
creating awareness (Jones et al., 2010). The increased reluctance of African American men toward prostate cancer screening may be due to a relationship with sexual stigma that is modified with the awareness and educational programs (Jones et al., 2010; R. A. Jones et al., 2011). In the case of men with prostate cancer and consequent side effects, it can be anticipated that they will experience challenges to their QOL. Hence, despite the scarcity of research representing the experiences of men with prostate cancer from different racial/ethnic backgrounds, it can be anticipated that they may experience similar QOL challenges. For this reason, a study examining QOL in men with prostate cancer in a multiethnic region like Miami, Florida will help understand these men's responses to the disease.

\section{Quality of Life}

Quality of life (QOL) is a major area of research that has emerged in the last couple of decades that is related to prostate cancer. Consequently, several definitions and conceptualizations have been studied with different diseases and chronic conditions and in various age groups and ethnicities. QOL was considered to be an outcome of a chronic illness or disease progression. The World Health Organization (WHO, 1947) introduced QOL when they defined health in terms of physical, mental, and social wellbeing. In the late 1970s, psychosocial care and QOL were identified as a patient's right in healthcare. This was also emphasized by the FDA when they favored QOL and enhanced survival as acceptable and preferred outcomes when considering the approval of drugs (Johnson \& Temple, 1985). This emphasis led to the emergence of research on the effects of psychological, social, environmental, and other factors on QOL in healthcare. 
QOL has been defined by many sources. It has been agreed that QOL is a multidimensional concept with a subjective nature (Cella, 1994; U.S. Department of Health and Human Services, 1990; Zhan, 1992). WHO (1993) defined QOL in terms of the individuals' perceptions of their lives accounting for cultural, social, and environmental circumstances. The nursing literature has emphasized the importance of defining and studying QOL. Accordingly, several definitions and models have been tested in a variety of populations along with several variables including disease specific, race/ethnicity, and other variables. Ferrans and colleagues's (2005) definition of QOL as the normal functioning, social usefulness, general well-being, ability to fulfill life's goals and happiness, and life satisfaction guides the conceptualization of this proposal (Ferrans et al., 2005). Initiated by Padilla and Grant's (1985) model of the relationship between the nursing process and the dimensions of QOL, the importance of embarking on studying men with prostate cancer emerged. The role of nursing research in contributing to the understanding of different dimensions of QOL is essential to enhance life satisfaction. In the case of men with prostate cancer, the disease and treatment impose side effects that impact the physical, psychological, and social wellbeing of men.

Weber and colleagues (2007) investigated the relationships between post radical prostatectomy urinary, sexual, and bowel dysfunction and the resultant bother to men. A sample of 72 men was recruited and surveyed 3 to 6 months after surgery. Self efficacy, social support, uncertainty, and physical function, and bother were measured. The majority of the sample (83\%) were White and married (73.6\%) with an average age of $59.8(S D=6.8)$ years. Sexual functioning scored lowest followed by urinary and bowel functioning. Upon examination of symptom bother scores, urinary symptoms scored the 
highest followed by sexual and bowel symptoms, respectively. There was no significant correlation between sexual function and bother. Conversely, there was a positive relationship between urinary function and bother $(r=.74, p<.001)$. Similarly, there was a positive relationship between bowel function and bother $(r=.05, p<.001)$. Men in this study reported having low self efficacy regarding treatment side effects. The majority of men (57\%) had low to moderate social support despite most of them being married. Most men (75\%) had moderate to high uncertainty about their disease, diagnosis, and treatment. Findings from this study showcased the physical functioning and resultant bother after undergoing radical prostatectomy (Weber et al., 2007). Findings from this study also made a unique contribution to the knowledge of the experience of men with prostate cancer. Nevertheless, findings cannot be generalized to the population because the sample size.

The physical and psychosocial factors that affect QOL in men 12 to 24 months after radical prostatectomy were examined (Rondorf-Klym \& Colling, 2003). The investigators hypothesized that the principle bodily functions affected by radical prostatectomy treatment were sexual and urinary functions. These functions were thought to contribute to changes in urinary and sexual appraisal which in turn affected men's psychosocial responses. With a sample of 88 Caucasian men diagnosed with prostate cancer, path analysis was conducted to test the hypotheses. Findings showed that sexual and urinary functioning predicted sexual appraisal. Similarly, urinary function predicted urinary appraisal. Urinary appraisal predicted health locus of control. Self esteem, perceived social support, and health locus of control were mediating variables and predicated QOL. Age predicted sexual function. There were no 
relationships between sexual and urinary appraisal and self esteem, anger suppression, perceived social support, and depression. There was also no relationship between sexual appraisal and health locus of control (Rondorf-Klym \& Colling, 2003).

Sexual and urinary dysfunctions have been documented to be primary concerns following prostatectomy. Gralnek, Wessells, Cui, and Dalkin (2000) studied the impact of nerve sparing techniques on QOL after radical retropubic prostatectomy for prostate cancer. Data were collected from 129 men at least 1 year after radical retropubic prostatectomy. The majority of the sample $(n=83)$ underwent nonnerve sparing surgery, and the rest $(n=46)$ were treated with unilateral nerve sparing prostatectomy. Men in the nerve sparing group scored significantly higher on the sexual function, sexual bother, physical function, and physical limitation domains of the RAND/UCLA Prostate Cancer Index. The nerve sparing procedure did not have a significant effect on urinary functioning, bowel functioning, and disease outcomes (Gralnek et al., 2000). Findings from this study are important to consider despite the rather small sample size. Findings suggested that despite the enhanced sexual functioning outcomes, the nerve sparing surgery was not found to decrease the other side effects of the surgery. As a result, men after nerve sparing prostatectomy still suffer from urinary functioning, bowel functioning, and disease outcomes. Therefore, this surgical technique may not enhance QOL outcomes in men with prostate cancer.

Brandeis, Litwin, Burnison, and Reiter (2000) compared general and disease specific health related QOL in men undergoing either brachytherapy or radical prostatectomy. In a sample of 48 men treated with brachytherapy and 74 men treated with surgery, health related QOL data were collected 3 to 17 months after treatment. The 
brachytherapy and prostatectomy groups were matched with a healthy control group from the literature. Findings showed that the general health outcomes did not differ between the three groups. The brachytherapy patients reported bowel function and urinary symptoms that were worse than the control group but better than the prostatectomy group. Sexual functioning and bother were much worse in both treatment groups when compared with the healthy control group. Patients who underwent brachytherapy reported improvement in physical function, bodily pain, urinary function, and bother with time (i.e., after the first year of treatment) (Bradeis et al., 2000).

Monga et al. (2005) evaluated QOL in men diagnosed with prostate cancer who underwent ERBT. The study also investigated the relationships between QOL, depression, sleep disturbance, and fatigue. The sample consisted of 40 men whose ages ranged from 55 to 78 years and were predominantly married (70\%). The participants underwent ERBT over a period of 7 to 8 weeks. Data were collected at five time points: pretreatment, midtreatment, completion of treatment, 4 to 8 weeks follow-up, and long term (i.e., 12 months or more). Findings showed that $45 \%$ of men developed mild to moderate proctitis (i.e., infection of the prostate) during ERBT. There were no significant changes in functional status, social wellbeing, functional wellbeing, and relationship with physician. There was a significant decline in physical wellbeing at midtreatment, completion of treatment, and long term. Physical wellbeing scores increased at 4 to 8 weeks without reaching baseline levels. Similar findings were demonstrated on the Piper Fatigue Scale. There was a significant inverse relationship between physical wellbeing and fatigue. Overall, there was a long term improvement in QOL in men undergoing ERBT without reaching baseline status (Monga at al., 2005). 
Potosky and colleagues (2002) examined QOL outcomes in men who underwent ADT or no therapy. A sample of 661 men (i.e., 245 receiving ADT and 416 receiving no therapy) were recruited from the Prostate Cancer Outcomes Study. A cross-sectional analysis to compare men receiving ADT and no therapy was conducted analyzing health outcomes 12 months after the diagnosis of prostate cancer. The majority of the ADT group received LHRH agonists. Findings at baseline showed that men who received ADT were less likely to have an erection firm enough for intercourse, lower libidos, and less frequent sexual activity compared with the no therapy group. The analysis was conducted between changes in sexual function in the ADT group and no therapy group and examined men who reported good sexual function at baseline. A total of 444 (ADT group $=149$, no therapy group $=295)$ men reported some interest in sexual activity at baseline. One year later, $79(54 \%)$ men in ADT group and $35(13 \%)$ of men in the no therapy group reported no interest in sexual activity. A total of 311 (ADT group $=88$, no therapy group $=223)$ men were potent at baseline. The majority of men in the ADT ( $n=$ $68,80 \%)$ and no therapy $(n=60,30 \%)$ groups was impotent at 12 months. Men in the ADT group reported significantly more breast swelling (20\%) and hot flashes (58\%). Physical discomfort, physical limitations, and overall bother were significantly higher in men in the ADT group. Men in the ADT group reported significantly decreased physical role functioning and vitality 12 months after the treatment (Potosky et al., 2002). Findings from this study suggested that ADT, similar to surgery and radiation therapy, has a negative effect on sexual functioning and QOL outcomes in men with prostate cancer. 
The percentage of men who underwent primary or adjuvant radiation therapy with changes in sexual function from the beginning to end of therapy was described by Howlett and colleagues (2010). Seventy men who underwent primary or adjuvant radiation therapy were recruited to participate in the descriptive longitudinal study. Depression, anxiety, and QOL measures were administered. Based on a question about the illness impacting their sexuality, patients were categorized into four groups: no problem (i.e., score less than 5) at beginning and end of radiation, no problem at beginning and problem at end of radiation, problem at beginning and end of radiation, and problem at beginning and no problem at end of radiation therapy. A cutoff point of 5 on a scale of 0 to 10 scale on the sexuality question was used to identify the categories. The majority of the sample was Caucasian (79\%) and married or partnered (74\%) with a mean age of 67.1 years $(S D=7.8)$. The sexuality question showed that $39 \%$ of men had no problem at beginning and end, $10 \%$ had no problem at beginning and problem at end, $44 \%$ had a problem at beginning and end, and $7 \%$ had a problem at beginning and no problem at end. There was a significant difference in the sexuality item between baseline and end of radiation in the no problem at beginning and end of radiation, no problem at beginning and with problem at end of radiation, and problem at beginning and no problem at end or radiation therapy groups. Findings showed that there was a significant change in depression scores from baseline to end of radiation therapy depending on the sexuality group. There was a significant effect of anxiety in the sexuality group but time did not have an effect. Men without sexual problems at the beginning and end of treatment had lower anxiety and depression scores in addition to higher QOL scores than did men in other groups. Although men did not score high on the depression scale, there 
was a significant difference in their scores over time depending on their sexuality group, especially in the groups in which men sustained sexual problems at the end of therapy. Changes in sexual function affected physical, psychosocial, and social wellbeing of men on the QOL scale. Men with decreased sexual functioning scored lower on the psychological and social wellbeing subscales (Howlett et al., 2010).

Sanda and colleagues (2008) examined the determinants of health-related QOL after primary treatment of prostate cancer and measured the effects of these determinants on satisfaction with the outcome of treatment in patients and their spouses or partners. Data were collected from 1,201 men and 625 spouses or partners before and after radical prostatectomy, brachytherapy, or ERBT. Findings showed that patients who underwent brachytherapy complained of long-lasting urinary irritation as well as bowel and sexual symptoms. Prostatectomy patients reported urinary incontinence. These symptoms were exacerbated by obesity, large prostate, high PSA, and older age. African American men had lower satisfaction with overall treatment outcomes. Sexual QOL was affected in all treatment groups. In the radical prostatectomy group, men who underwent nerve sparing procedures had better sexual functioning when compared to the nonnerve sparing group. In the ERBT group, improvement in sexual functioning was better in men who received radiotherapy alone when compared with men who received radiation and ADT. Large prostate size, high baseline PSA, and older age were associated with worse sexual functioning. QOL scores were significantly associated with the degree of outcome satisfaction among men and their spouses or partners. Spouses or partners reported distress related to erectile dysfunction in $44 \%$ of the prostatectomy group, $22 \%$ of the radiation group, and 13\% of the brachytherapy group. Men in the brachytherapy and 
radiotherapy group reported having lower QOL related to bowel function up to 1 year after treatment. Bowel dysfunction was associated with distress reported by $5 \%$ of spouses or partners in the radiotherapy group and $4 \%$ in the brachytherapy group. Men's QOL was associated with the satisfaction with the spouse or partner (Sanda et al., 2008).

In a 2 year follow up study on men treated with prostate cancer, Penson and colleagues (2003) examined the relationships between primary treatment, urinary dysfunction, sexual dysfunction, and general health related QOL. Participants were selected from the Surveillance, Epidemiology, and End Results (SEER) registries. Baseline surveys were completed within 6 to 12 months of diagnosis. Men were followed for up to 2 years after the diagnosis, and health related QOL data were collected. Findings showed that primary treatment was not associated with 2-year general health related QOL outcomes. A total of 2,306 cases met the inclusion criteria of the study. Men in this sample were mostly White (78\%), 60 years or older (86\%), and married or had a sexual partner $(83 \%)$. At 24 months, men reported having a moderate or big problem with their sexual function (39.2\%) with no erection firm enough for sexual intercourse (65\%), especially in men undergoing radical prostatectomy. Urinary function was also described to be in the form of frequent leakage or total loss of control $(90.9 \%)$, especially for men who had hormone ablation therapy and radiation therapy. Urinary bother and sexual dysfunction significantly affected health related QOL. Urinary function was not to have a significant effect on health related QOL. Sexual dysfunction and bother contributed to a decline in the six domains of health related QOL. There was no significant primary treatment effect on health related QOL 2 years after the diagnosis of prostate cancer. Findings from this study suggested that irrespective of the treatment 
choice, long term QOL in men treated for prostate cancer is very similar (Penson et al., 2003).

Potosky and colleagues (2004) investigated 5 year QOL outcomes in men who underwent radical prostatectomy or radiotherapy for treatment of prostate cancer. Men ages 55 to 74 years $(n=1,187)$ diagnosed with localized prostate cancer and treated with radical prostatectomy or ERBT were included in the study. Data on clinical outcomes and QOL at baseline (i.e., upon diagnosis) as well as 2 and 5 years following treatment were collected. Five years after the diagnosis, sexual functioning declined (41-62\%) in both groups, especially in the domains of interest, frequency of any sexual activity, or the extent of bother due to sexual dysfunction. Men who underwent radical prostatectomy had a greater prevalence of erectile dysfunction ( $79.3 \%$ vs. $63.5 \%)$. Two years after the diagnosis of prostate cancer, men reported impotence after radical prostatectomy $(82.1 \%)$ and after ERBT (50.3\%). At 5 years, these percentages were $79.3 \%$ versus $63.5 \%$, respectively. Upon comparison of sexual functioning between 2 and 5 years, men who received ERBT had a significantly larger decline in their sexual function, whereas men undergoing surgery experienced a smaller decline. Men who underwent surgery were more likely to be incontinent at 5 years $(15.3 \%$ vs. $4.1 \%)$. Urinary incontinence had similar percentages at 2 years. It is worth noting that men who had ERBT reported a higher prevalence of pretreatment incontinence. Bowel dysfunction (e.g., bowel urgency, incontinent hemorrhoids) was common among men undergoing ERBT (Potosky et al., 2004).

Steineck and colleagues (2002) compared the QOL between men treated with radical prostatectomy and watchful waiting. Data on QOL in men with localized prostate 
cancer who participated in a large population based cohort study were analyzed. Three hundred and twenty six men were included in the study. Findings suggested that erectile dysfunction was reported by men treated with radical prostatectomy ( $80 \%)$ and men who were following watchful waiting (45\%). Urinary leakage (49\% and $21 \%)$ and urinary obstruction $(28 \%$ and $44 \%)$ were also reported, respectively. There were no differences in bowel function, anxiety, depression, well-being, and QOL between the two groups. Findings from this study suggested that there may be a difference in the treatment side effects between watchful waiting and surgery. However, there were no differences in wellbeing or QOL as shown in the 4 year follow up of the study (Steinek et al., 2002). In a comparison of the effects of radical prostatectomy $(n=1,156)$ and ERBT $(n$ $=435$ ) on urinary, bowel, and sexual functions on QOL, Potosky and colleagues (2000) conducted a 2-year follow-up study. Men ages 55 to 74 years old and newly diagnosed with prostate cancer who underwent one of the two treatments were recruited to participate in the study. Men who had radical prostatectomy were more likely to be incontinent $(9.6 \%$ vs. $3.5 \%, p<.001)$ and be impotent $(79.6 \%$ vs. $61.5 \%, p<.001)$. There was a significant decline in sexual functioning in both groups from before to after the procedure. However, men who underwent radiation therapy reported having a significant decline in bowel function. There was no significant difference in the health related QOL outcomes between the two groups (Potosky et al., 2000).

Chen, Clark, and Talcott (2009) reported QOL outcomes of 409 patients 36 months after radical prostatectomy, external beam radiation, and brachytherapy. Findings showed that the greatest increases in erectile dysfunction were found in men with normal baseline function. Men with poor baseline urinary obstruction or irritation 
reported an improvement in their symptoms after treatment, especially surgery. Findings from this study compared patient outcomes to baseline functional status. This comparison helps explain the impact of prostate treatment according to the specificity of each man's condition. Results highlight the importance of baseline functioning on patient outcomes up to 36 months after treatment (Chen et al., 2009).

To explore the uncertainty, anxiety, and the health related QOL and affective QOL in men who underwent watchful waiting, a descriptive study was conducted with 21 men diagnosed with prostate cancer. The age range of men in this study was 65 to 85 years $(M=76, S D=6.71)$ who were diagnosed with prostate cancer for an average of 4.5 years ( $S D=33.89$ months). Correlational analyses between anxiety, uncertainty, and danger appraisal as independent variables and health related QOL were not significant. Hierarchal regression analysis showed that uncertainty explained $36 \%$ of the variance in affective QOL. Adding anxiety and uncertainty to the model explained $60 \%$ of the variance. Danger appraisal played a mediating effect between uncertainty and affective health function of QOL (affective health function is one of the domains measured in health-related QOL). Affective health function was the only QOL domain significantly related to the independent variables. Findings from this study suggest that men engaging in watchful waiting as a treatment for prostate cancer were uncertain. Uncertainty contributes to their perception of danger and influences affective QOL. Despite the small sample, findings support the uncertainty experienced by men with watchful waiting as a treatment (Wallace, 2003). Generalization of these findings cannot be made, but the effect of uncertainty on QOL in men with prostate cancer is suggested. 
A qualitative study to explore the influence of erectile dysfunction on men who had undergone definitive treatment for early nonmetastatic prostate cancer was conducted using seven focus groups. A total of 48 men treated for early prostate cancer for 12 to 24 months were included in the study. Men's experiences and QOL concerns related to prostate cancer and its treatment were discussed in the semistructured interviews. The extracted themes identified the impact of sexual dysfunction on the QOL in these men. Four themes were identified: the qualities of sexual intimacy; everyday interactions with women; sexual imagining and fantasy life; and men's perceptions of their masculinity. Discussions suggested that sexual dysfunction had an effect on the men's intimate relationships and the way they perceived themselves as sexual beings (Bokhour, Clark, Inui, Silliman, \& Talcott, 2001).

Korfage and colleagues (2006) explored the discrepancy between levels of erectile, urinary, or bowel dysfunctions and generic QOL scores. In a qualitative study to explore men's perceptions of the side effects of prostate cancer treatment, 33 men were interviewed using a semistructured guide. Eighteen interviews were conducted asking men about their health and how it was affected by prostate cancer. The aim of the study was to further explore findings from a quantitative study that suggested high QOL scores despite the high prevalence of side effects (i.e., sexual, urinary, and bowel dysfunctions). The participants were randomly assigned to two groups. The first group was interviewed to determine if the shift in responses was the result of their adaptation of health change. The second group explored the possibility of generic measures being insensitive to changes in health status after the treatment of prostate cancer. Findings from this study suggested that age of men in the sample influenced the degree of considering the side 
effects to be a major issue affecting their health. Men in their 70s accepted their sexual dysfunction and considered being alive a priority for them. Urinary incontinence was considered more of an issue since it made them dependent on others. Older age of men contributed to their acceptance of sexual dysfunction as a side effect of prostate cancer. The rationalization of old age and aging process was used by men to help accept their condition. Men talked about getting used to their problems. They explained side effects in terms of a consequence or inconvenience that resulted from a life saving procedure. However, when they compared themselves to other men in their 70s age category, they were thankful to be rather healthy. Trying to adapt to their situation and the corresponding changes in their life was their main focus. Since they perceived these side effects would accompany them for a long time, men were trying to accept their new condition. As a result, they did not consider impotence and urinary leakage as health problems and therefore scored higher on the health related QOL measure (Korfage et al., 2006). Findings from this study showed that men with prostate cancer symptoms may reach acceptance of their conditions. However, the study was conducted in the Netherlands, and findings may not be applicable in different cultures and settings. In summary, the diagnosis of prostate cancer has inevitable side have side effects of sexual dysfunction and urinary incontinence (Penson et al., 2008; Potosky et al., 2000; Stanford et al., 2000; Wilt et al., 2008). These side effects affect men's sexuality and QOL (Penson et al., 2003). Research that has used data from the Surveillance, Epidemiology, and End Results (SEER) registries has shown that sexual dysfunction contributed to a decline in QOL irrespective of the treatment choice (Penson et al., 2003). Other studies have highlighted the impact of different prostate cancer treatments on 
sexual and urinary function and the consequent effect on QOL. For example, Howlett et al. (2010) indicated that here was a significant change in depression and QOL scores between before and after radiation therapy in men without sexual problems. Changes in sexual function affected physical, psychosocial, and social wellbeing of men on the QOL scale (Howlett et al., 2010). Sanda and colleagues (2008) also reported that men who underwent brachytherapy and radiotherapy reported having low QOL. Men's QOL was found to be associated with satisfaction with the spouse or partner (Sanda et al., 2008). In this study, African American men had lower satisfaction with the overall treatment outcomes. Also, older age was associated with worse sexual functioning (Sanda et al., 2008). Men who opted to undergo watchful waiting for prostate cancer progression were found to be uncertain due to perceptions of danger that may affect their health; therefore, their QOL was affected (Wallace et al., 2003).

As a result of side effects, men's sexuality is compromised due to the decreased sexual abilities. Thus, men feel distressed and try to maintain their perceived socially constructed and expected role (Chapple \& Ziebland, 2002; Gray et al., 2002; Hawkins et al., 2009). Men strive to save their personal image as a man and try to maintain their image in front of their partner (Wall \& Kristjanson, 2005). Consequently, their masculinity and partner relationships are challenged and their QOL may be affected (Burns \& Mahalik, 2006; Chapple \& Ziebland, 2002; Fergus et al., 2002).

Studies have not examined the contributions of age, ethnicity, treatment side effects (i.e., sexual dysfunction and urinary incontinence), masculinity, and men's perception of their partner relationship together for their independent and combined contribution to QOL in men with prostate cancer. Studies have examined the 
contributions of these variables independently (Burns \& Mahalik, 2006; Chapple \& Ziebland, 2002; Fergus et al., 2002; Penson et al., 2008; Potosky et al., 2000). Also, masculinity has mostly been studied using qualitative research designs (Hawkins et al., 2009; Gray et al., 2002; Oliffe, 2005). Likewise, the majority of studies have been conducted with samples that were predominantly Caucasian or nonHispanic White men. With Miami, Florida hosting a large Hispanic and Latin American community that emphasizes the values of machismo, examining QOL in men with prostate cancer society will contribute to the literature and to the quality of healthcare. 


\section{CHAPTER III \\ RESEACH DESIGN AND METHODS \\ Design}

This study used a cross-sectional design to examine the contributions of age, ethnicity, sexual dysfunction, urinary incontinence, masculinity, and perception of relationship with the partner on QOL in men diagnosed with prostate cancer. The crosssectional design is used to capture data about men with prostate cancer at one period of time. This method is appropriate for studies whose purpose is to describe the status of a phenomena or relationships between concepts at one point in time (Polit \& Beck, 2012). In this study, the purpose was to examine relative contributions of age, ethnicity, sexual dysfunction, urinary incontinence, masculinity, and perception of the relationship with the partner on the quality of life in men diagnosed with prostate cancer. Thus, a crosssectional design was appropriate for the purpose of the study. Men with prostate cancer were recruited by the principal investigator (PI) who has had clinical experience working with oncology patients.

\section{Setting}

Men diagnosed with prostate cancer were recruited from four urology clinics in Miami and Broward counties in FL. Potential participants were identified by staff at the clinics and support groups.

Clinic 1 is a urology clinic that provides excellent urologic care to residents of south Florida and the surrounding region. The clinic physicians treat patients with a variety of urologic disorders such as kidney stones, benign prostatic hyperplasia (BPH), prostate cancer, bladder cancer, urinary incontinence, sexual dysfunction, and recurring 
urinary infections. Clinic physicians diagnose and treat around 300 men with prostate cancer annually using surgery, hormonal therapy, and watchful waiting. Clinic 2 is a clinic that delivers quality and innovative urologic care since 1968. The clinic physicians care for patients with infections, urinary incontinence, male sexual dysfunction (i.e., impotence), male infertility and minimally invasive surgery (MIS) of the urinary tract. More than 250 men with prostate cancer are annually cared for at the clinic.

Clinic 3 is known for their latest innovative urologic procedures and diagnostic Studies include robotic and laparoscopic surgery, laser prostate surgery, flexible uretersoscopy, minimally invasive incontinence correction surgery, and nerve-sparing prostatectomy. The physicians at the clinic attend to a diverse population with urinary symptoms, prostate cancer, kidney stones, urinary incontinence, erectile dysfunction, bladder cancer, kidney cancer, testicular cancer, urinary tract infections, infertility, and vasectomy. The clinic serves more than 200 men with prostate cancer yearly. Clinic 4 is a clinic that provides care for patients with a multitude of urologic symptoms and diseases. The clinic also has a new procedure, Evolve Laser of the Prostate. Around 200 men with prostate cancer are estimated to be cared for by this clinic each year. These clinics are considered to be major centers in South Florida, and they both serve a multicultural patient population that mirrors the population of Miami and includes Hispanics (e.g., Cuban, Columbian, Venezuelan, Chilean, Peruvian), African Americans, Caribbean, Haitians, Caucasians, and men from other ethnicities.

\section{Sample}

Men diagnosed with prostate cancer who self-identified as being heterosexual and partnered were recruited for participation in the study. Men self who identified 
themselves as men who have sex with men were excluded for the purpose of this study. Gay men have been identified to have a different experience with decreased sexual functioning and relationships with partners (Blank, 2005). Men in the sample were required to be English or Spanish speaking and able to communicate with the researcher and research assistants as well as read and respond to the study instruments. Study instruments and the consent form were available in English and Spanish according to the preference of the participant. The duration since the diagnosis or treatment and the length of the relationship with the partner was not specified in the inclusion criteria in order to not limit the number of men eligible for participation in the study. Men with erectile dysfunction, urinary incontinence, or bowel dysfunction due to reasons other than prostate cancer were excluded from the study.

\section{Power Analysis}

Power analysis was conducted for study analyses used G*Power 3.1 (Faul, Erdfelder, Lang, \& Buchner, 2007). G*Power is a statistical program used for a priori and post hoc analysis (Faul et al., 2007; Faul, Erdfelder, Buchner, \& Lang, 2009). A priori sample size estimation is essential to determine an adequate sample size for a specific statistical analysis (i.e., correlational analysis, regression analysis) (Cohen, 1988;

Cohen, Cohen, West, \& Aiken, 2003). For this study, power analysis based on regression analysis to answer research question two was conducted with effect size of .15 (i.e., medium effect), power of .80, and five predictors (Cohen, 1988). Pearson $r$ correlation and Chi Square analysis were conducted to answer research question one. A priori analysis to determine the adequate sample size needed to conduct correlation analysis using $t$ test family with five variables was computed. Effect size was identified to be .3 
(i.e., medium effect) with a desired power of .80 and significance level set at .05 . A sample size of 64 men was identified to be adequate for the analysis. This was also similar to conducting Chi Square analysis with two variables. A sample of 92 men was identified to be adequate to conduct regression analysis. Significance level (i.e., alpha) was set at .05. Moderate effect sizes were selected since the instruments used to measure the variables are valid, reliable, and have been widely used. Therefore, it is anticipated that these instruments will be able to measure each of the identified concepts appropriately (Mertler \& Vannatta, 2013). Due to the sensitivity of the subject, it was projected that $25 \%$ of the surveys may not be completed or returned. As a result, a total sample of 123 men meeting the inclusion criteria of the study was recruited. A total of 107 men completed the study questionnaires, hence rendering the response rate to be $87 \%$.

At the end of the study, post hoc analysis was conducted to determine the actual power attained. Post hoc analysis to conduct correlation analysis using $t$ test family with five variables was computed and a sample of 107 men living with prostate cancer; effect size was identified to be .3 (i.e., medium effect), and significance level set at .05 . The power was 0.94. This was also similar to conducting Chi Square analysis with two variables. A power of 0.87 was identified in the post hoc analysis for regression analysis with significance level set at .05 and five predictors.

\section{Pilot}

The study instruments were available in English and Spanish according to the preferences of the participants. The English and Spanish versions of the instruments have been used and validated. The psychometric properties of the instruments have been 
published. The validity and reliability of the instruments will be discussed in a later section. The majority of the Spanish versions of the instruments were validated in Hispanic populations in the South West and North East of the United States where the Hispanic community is predominantly Mexican or Puerto Rican. However, the Hispanic population in Miami is predominantly Cuban, Columbian, and other Caribbean and Latin American ethnicities. In order to ensure the comprehension and legibility of instrument items during the study, a pilot study including five men who resided in the community and who met the eligibility criteria was conducted. Also, the pilot provided information about the estimated amount of time needed to complete the instruments.

The pilot study participants were asked to read the demographic form and study instruments and identify understandability, clarity, and cultural appropriateness of the items. These participants were required to identify items that may not be understood. The results of the pilot were reviewed by the principle investigator (PI) as well as dissertation chair and committee. Based on findings from the pilot study, modifications were made. The purpose of the pilot study was to ensure the cultural and linguistic appropriateness of the Spanish version of the study questionnaire.

\section{Sampling and Recruitment}

Writings about the recruitment of men in research studies has discussed the experiences of researchers and addressed issues to be considered when designing a men's health study. Yancy, Ortega, and Kumanyika (2006) discussed recruitment strategies that include mass mailing to African American men especially men of middle to higher socioeconomic status. The authors did not support population based sampling since it was reported to produce insufficient samples from required ethnic minorities. 
Community involvement survey responses were found to be effective when followed with telephone follow up with African American men. Cultural adaptation of the recruitment strategies was the utmost important consideration that encouraged men to participate in studies (Yancy et al., 2006). Cultural adaptation of recruitment strategies and cultural sensitivity were also discussed by Woods, Montgomery, and Herring (2004). The authors emphasized the importance of understanding the acceptable language, terminologies, and approach to the concepts being studied. For example, for this study, sexual dysfunction and prostate cancer are considered to be sensitive topics to men in general and Hispanic men in specific. Therefore, recruitment strategies refrained from referring to participants as having sexual or prostate problems in order to encourage men to participate in the study without being identified as having a condition that would affect their masculine image. S. G. Jones and colleagues (2011), on the other hand, emphasized the importance of conducting focus groups and discussions with men who met eligibility criteria to share the appropriate recruitment strategies. Their discussion was generally focused on the recruitment of Hispanic men in a sexual behavior study. Recruitment from urology or specialized clinics and clinicians was also advised. The length of the survey and the appropriate incentives were important considerations (S. G. Jones et al., 2011). Word of mouth was also considered to be a successful strategy in the recruitment of African American men (Jones, Richard, \& Ishan, 2009).

As a result, the strategies for the recruitment of men for this study included primarily collaborating with urology clinics, support groups, physicians, and clinicians to identify potential participants. The support of the main physicians at the urology clinics and support groups encouraged men to participate in the study. The support of the 
physicians, nurses, nurse practitioners, physician assistants, and clinic staff, figures of authority was thought to provide trust and confidence to men and therefore increased the likelihood of their participation in the study. The recruitment material referred to the study as the "Men's Health Study" (S. G. Jones et al., 2011). Meetings and communication with the PI, dissertation chair, and committee members included discussions of the effectiveness of strategies for recruitment of study participants. Accordingly, strategies were maintained and modified to reach the recommended sample size within the allotted time frame.

Meetings were conducted at the clinics and support group meeting places to explain the objectives and procedure of the study to the physicians and staff. The purpose of these meetings was to familiarize the physicians and staff with the study to support the PI by referring potential participants. Potential participants were referred to the PI from the pool of men attending these clinics and support groups. These participants were diagnosed and treated for prostate cancer prior to participating in the study. The PI then contacted the potential participants to recruit them for the study and secure their informed consent. After the informed consent was obtained, data were collected from men using paper and pencil questionnaires with the study instruments. To ensure confidentiality of participants, a private room/office was provided at each of the clinics for men to be able to conveniently and privately complete the study instruments. Data regarding disease stage and treatment were collected using a demographic data form that was provided to the participants with the study instruments. 


\section{Procedure}

Institutional Review Board (IRB) approval for the study was obtained from Florida International University (FIU) to ensure ethical standards were met. Letters of support were obtained from the clinics and support groups. Informed consents included information about the study objectives and contact information for the researcher in addition to potential risks and benefits of the study based on the FIU IRB policies and procedures. The PI and dissertation chair completed the required human subjects' research training. Research assistants were also required to complete the required human subjects' research training (i.e., CITI).

Men with prostate cancer were referred to the PI as potential study participants by staff at the urology clinics and support groups. The clinic staff distributed study flyers with the name of the study, the PI, and contact information in case the potential participants wanted to contact the PI. The PI met potential participants at the urology clinics and support group meeting sites and introduced the "Men's Health Study" as well as explained the study objective, procedure, and potential risks and benefits. The PI screened the men for eligibility and asked them about their interest in participating in the study. Men who elected not to participate in the study were thanked and ensured that their decision would not affect the course of their treatment at the clinic. No further contact with these men was made. Men who choose to participate in the study and who met the eligibility criteria met with the PI in a private room. Men at the clinic who learned about the study from flyers and clinic staff were also approached by the PI. The PI screened the men for eligibility and explained the study objective, procedure, and potential risks and benefits. The same procedure was followed thereafter. 
The clinic staff was informed about enrollment of potential participants to facilitate the arrangement of a room for the identified appointments. At the clinic, a quite room was provided for the PI and the participant to ensure privacy and confidentiality. The study objectives, purpose, procedure, benefits, and potential risks were reviewed with the potential participant. The informed consent was read to the potential participant. After reading and explaining the informed consent to the potential clients, the PI assessed the client's understanding of the purpose of the study. The client was asked to describe or answer specific questions about the risks, anticipated benefits, research purpose, and/ or voluntariness issues. This was thought to assess the client's ability to give informed consent. If the PI suspected that the potential participant had problems understanding consent-related issues, the participant was not enrolled in the study for a potential impaired ability to consent. Ensuring the participant's ability to demonstrate an adequate level of understanding of the consent is paramount to comply with research ethics standards (U.S. Department of Health and Human Services, 2009).

The study questionnaire was provided to participants after the informed consent form was signed. The study questionnaire included a demographic data form, the University of California Los Angeles Prostate Cancer Index (UCLA PCI), Conformity to Masculine Norms Inventory (CMNI), Dyadic Adjustment Scale (DAS), and Quality of Life (Short Form Health Survey version 2.0 [SF36v2]) instruments. Men were asked to return the completed questionnaire to a sealed box. If men needed assistance reading the questionnaire items, the PI read the items and wrote participants' answers to the items. Participants were asked not to include their names or any identifiers on the study questionnaire. Questionnaires and informed consent were available in English and 
Spanish depending on the language of preference of participants. Data about the disease (i.e., stage of the tumor, PSA levels, Gleeson score, treatment option, and presence of comorbidities) were collected as part of the demographic data form.

It was estimated that the data collection process required 20 to 30 minutes to be completed. Participating men were allowed time for breaks during data collection to ensure comfort and completion of the process. Data collection in the urology clinic before and during the waiting time of the appointment facilitated the process for participating men. Men recruited from the support groups completed the questionnaires after the completion of the meeting. The clinic and support groups were considered to be safe and comfortable environments that would help men be at ease when completing sensitive questions about sexual dysfunction, urinary incontinence, masculinity, partner relationship, and QOL. The presence of the PI during data collection allowed participants to ask questions and clarify items of the questionnaire. Upon completion, the participants returned the questionnaires to a sealed box that was emptied weekly by the PI. This was thought to promote comfort and maximize confidentiality.

Participation in the study posed a minimal risk for men. A potential risk was the psychological distress that may be experienced by men as a result of sensitivity of the concepts included in the questionnaires. Men were provided with a list of resources for psychological assistance and counseling in the Miami and Broward metropolitan areas. The questionnaires were self administered and therefore could be completed by men without the presence of the PI. Therefore, the list of resources was included as part of the package that included the consent form and questionnaires. During the recruitment process, the PI discussed the resource list in case psychological assistance was needed. 
Also, the PI is an experienced nurse in the caring for oncology patients and cancer survivors who has undergone training regarding working with this population.

Due to the nature and scope of the study, there were no anticipated physical, social, or legal risks. Breech in confidentiality was also prevented through the anonymity of the research packages, and data were kept in secure and locked files. The informed consent was separated from the study questionnaires so as to preserve confidentiality. Protection against breach of confidentiality was ensured through use of identification numbers for each participant. No names or information that would identify the participants were included in the return packages. There was no list of participants or names of participants provided to the PI so as to ensure privacy and confidentiality. The handling of the questionnaires and the data was solely by the PI and dissertation committee chair and members who have completed the CITI human subject training. Data entry and coding included identification numbers, and data were handled by the PI. Dissemination of the study findings included aggregate data and did not include any information that will reveal the identity of the participants. Demographic data were presented in terms of frequencies and percentages for nominal level variables and descriptive statistics for ordinal and interval level variables.

The potential benefit of participating in the study was to broaden knowledge about the experience of men with prostate cancer from minority racial/ethnic groups. While answering the questionnaires, men may think about the issues related to their prostate cancer treatment, masculine norms, and relationships with their partners, and QOL. These thoughts may encourage them to use the provided psychological resources to seek help or support. The resources provided may also provide men with a venue to 
enhance their QOL. There was no projected harm that was anticipated from participation in the study.

\section{Measures}

The study included five independent variables and one dependent variable. The variables were measured using valid and reliable instruments.

\section{Independent Variables}

The independent variables were age, ethnicity, sexual dysfunction, urinary incontinence, masculinity, and relationship with the partner.

Demographic and Background Variables. Demographic and background data were collected by items on participant age, race/ethnicity, SES, sexual history, time since diagnosis, relationship status (e.g., married, cohabitating), and relationship duration. Data were also gathered on education, employment status, and number of children, if applicable. Data on the stage of the tumor, PSA levels, Gleeson score, treatment option, and presence of co-morbidities were collected as part of the demographic data form and were used to describe the sample. Participants were also asked about sexual enhancement regimens with an item on the survey.

Data on the presence of co-morbidities were collected, and the relationship between presence of co-morbidities and QOL was analyzed. In case of the presence of a strong relationship, controlling for the presence of co-morbidities was considered. Patient-centered care provision was assessed using three questions in the demographic that asked about coming to the decision of treatment, degree of inclusion of patient preferences, and whether patient concerns were taken into consideration prior to making the treatment decision. These data were used to further describe the sample. 
Prostate cancer treatment side effects. Prostate cancer treatment side effects (i.e., sexual dysfunction and urinary incontinence) were measured using the University of California Los Angeles Prostate Cancer Index (UCLA PCI) (Litwin et al., 1998). The majority of instruments used to assess prostate cancer treatment side effects also measure quality of life. The European Organization for Research and Treatment of Cancer Quality of Life Questionnaire- Prostate Cancer (EROTC PR-25), Functional Assessment of Cancer Therapies- Prostate (FACT-P), Expanded Prostate Cancer Index Composite (EPIC), International Prostate Symptom Score (IPSS), and International Index of Erectile Function (IIEF) have been used to assess sexual dysfunction and urinary incontinence in men with prostate cancer. EROTC PR-25 is a 25 -item instrument that assesses erectile, bowel, and urinary function, however it has not been widely used. FACT-P is a 34-item questionnaire that assesses weight loss, role, erectile, and lower urinary tract symptoms after prostate cancer; however, the measure may not be sensitive to early prostate cancer symptoms. EPIC is a 50-item instrument that measures prostate cancer treatment side effects and is considered to be an expanded version of the UCLA PCI. IPSS and IIEF measure erectile function and lower urinary tract symptoms (i.e., storage, voiding, post micturition) without assessing the relationship with the quality of life of men. UCLA PCI is one of the most widely used instrument that has been translated and validated in Spanish.

UCLA PCI is composed of 15 -items that assess outcomes of men with prostate cancer in three domains: urinary function, bowel habits, and sexual function. The items are rated on 3 or 5-point Likert scales (i.e., there are different ratings for each question). The overall score ranges from 0 to 100 , and the higher the score, the better the identified 
health status. The test-retest reliability estimates of the urinary and sexual functioning subscales over a 2 week period were reported to be .93 and .92 , respectively. The internal consistency estimates of the subscales were .87 and .93 , respectively (Litwin \& McGuigan, 1999). Construct validity was also supported for the English version of the scale by correlating the results with other well established quality of life scales (Litwin \& McGuigan, 1999). The instrument was also successfully tested for its responsiveness to changes in QOL in men with prostate cancer (Bergman, Saigal, Kwan, \& Litwin, 2010). Studies have used the UCLA PCI to measure the extent of symptom bother (Weber et al., 2007) and health related QOL (Anger et al., 2007) of men with prostate cancer. The UCLA PCI was translated into Spanish and validated on a sample of men $(n=100)$ recruited from Hialeah, Florida. The majority of men were born in Puerto Rico or Cuba in addition to other Latin American countries and Spain. Reliability estimates documented that kappa was at least .81 for all items. The items related to health and social interactions showed poorer reliability estimates. The authors explained the poor reliability of these items to be related to the fact that men participating in the study were in good health and socially active (Krongrad et al., 1997). Findings from study indicated favorable reliability estimates for the English and Spanish versions. The authors suggested that the sample included a variety of Spanish dialects and therefore the instrument may be used with Spanish speaking men from a variety of Latin American countries (Krongrad et al., 1997).

Masculinity. The Conformity to Masculine Norms Inventory (CMNI) was used to measure masculinity. Other instruments that have been used to measure masculinity in other studies are Bem Sex Role Inventory, Macho Scale, Gender Role Conflict Scale, 
Eisler's Gender Role Stres Scale, Brannon Masculinity Scale, Male Role Norms Scale, Male Role Norms Inventory. The Bem Sex Role Inventory and the Macho scale assess masculinity in a unidimensional manner without focusing on the complex and multidimensional nature of the concept. The other instruments measure conflicts with masculinity and did not focus on the perception and adherence to masculine norms.

CMNI was devised by Mahalik and colleagues (2003) to measure the socially constructed norms and the conformity of men to masculine behaviors. The original multidimensional tool consisted of 94 items and 11 subscales (i.e., winning, emotional control, risk-taking, violence, dominance, playboy, self reliance, primacy of work, power over women, disdain for homosexuals, and pursuit of status) that measure conformity to masculine norms. The items scales are answered on a 4-point Likert scale ranging from 0 (strongly disagree) to 3 (strongly agree). Scores on the inventory range from 0 to 282 with higher scores indicating higher conformity to norms of masculinity. Internal consistency estimates for the original tool were .72 to .91 for the subscales and .94 for the total scale. Test-retest reliability estimates over a 2 to 3 weeks period ranged from .75 to .95 for the subscales and .96 for the total inventory. Validity was also examined using exploratory factor analysis (Mahalik et al, 2003).

Permission for the use of this tool was secured from the author. This author also has a short version of the CMNI that includes 22 items which was used in this study. The CMNI was used to examine the conformity of masculine norms and health related behaviors with men with spinal cord injury (Schopp, Good, Mazurek, Barker, \& Stucky, 2007), traumatic brain injury (Schopp, Good, Barker, Mazurek, \& Hathaway, 2006) and perceived normative health behaviors (Mahalik, Burns, \& Syzdek, 2007). This tool was 
used to measure masculinity in adult men with prostate cancer. The details of the studies have been previously discussed in the literature review. The CMNI was translated to Spanish; however, the psychometric estimates for the Spanish version have not been published.

Relationship with partner. Relationship with partner was measured using the Dyadic Adjustment Scale (DAS), a 32-item self report instrument (Spanier, 1976). Other instruments that measure relationship with the partner considered include the Multidimensional Relationship Questionnaire (MRQ) and Measure for Marital Satisfaction (MMS). However, the decision to use the DAS was based on the fact that the majority of cancer studies have used this instrument for assessing the relationship with the partner.

The DAS has been used with married and cohabitating couples; thus, it is appropriate for this study of partnered men irrespective of their marital status. It measures the relationship satisfaction using four subscales: satisfaction, cohesion, consensus, and affectional expression. The items are scored on a 6-point Likert scales ranging from 0 to 5 with variable anchors. Total scores range from 9 to 151 where a total score below 100 indicates marital distress and higher score reveal better relationship functioning (Spanier, 1976). Cronbach's alpha internal consistency for the total scale was reported to be .92 , and internal consistency estimates for the subscales ranged from .73 to .94 (Spanier, 1976). The DAS has been used to measure the quality of a dyadic relationships in many populations including patients with prostate cancer (Badr \& Carmack Taylor, 2009; Banthia, Malcarne, Varni, Ko, Sadler, \& Greenbergs, 2003; Wootten et al., 2007). The DAS was translated to Spanish and validated on a sample of 
Hispanic Americans in Miami $(n=78)$. The internal consistencies were reported to rage from .67 to .93 , and test retest estimates after 2 weeks ranged from .79 to .87 . Also, there were high correlations between the English and Spanish versions (.91 to .99) (Youngblut, Brooten, \& Menzies, 2006).

\section{Dependent Variable}

The dependent variable was quality of life of men with prostate cancer. QOL in men with prostate cancer was measured using the 36-item Short Form Health Survey version $2.0(\mathrm{SF}-36 \mathrm{v} 2)$ that measures the overall health status, functional status, and health related QOL (Ware, Kosinski, \& Dewey, 2000; Ware, Kosinski, \& Gandek, 2000). The Expanded Prostate Cancer Index Composite (EPIC), EROTC-PC, FACT-P, Prostate Cancer-specific Quality of Life Instrument (PROSQOLI), Prostate Cancer Treatment Outcomes-Questionnaire (PCTO-Q), Patient Oriented Prostate Utility Scales (PORPUS), Prostate Cancer Related Quality of Life, and Prostate Cancer- Quality of Life (PC-QOL) are other instruments that were used to measure QOL in men with prostate cancer. The SF 36v2 is a widely used instrument that measures QOL providing information about the physical and mental health functioning of men with prostate cancer.

SF-36v2 includes eight subscales: physical functioning, role physical, bodily pain, general health perceptions, vitality, social functioning, role emotional, and mental health (Ware, Kosinski, \& Dewey, 2000; Ware, Kosinski, \& Gandek, 2000). These subscales include physical and mental health component summary measures. The SF-36 was developed using concepts from the Medical Outcomes Study that represent the major concepts used in health surveys and affected by disease and treatment (Stewart \& Ware, 1992; Ware, 1995). 
The SF-36 version 2.0 is an enhanced version of the survey that was documented to be simplified with shortened items. The instrument was shown to have greater comparability between the translated versions and demonstrated good cultural adaptations (Ware et al., 2000). The SF-36 is available in standard (i.e., 4-week) and acute (i.e., 1 week) recall (Ware et al., 2000). The standard version was used for this study. The SF-36v2 consists of 36 items that are scored on a 5-point Likert scale with the exception of the physical functioning subscale that is scored on a 3-point Likert scale (Ware et al., 2000). The overall score ranges from 0 to 100 in which higher scores indicate better health status and QOL (Ware et al., 2000). Factor analysis confirmed the two components of physical and mental health accounting for $80 \%$ to $85 \%$ of the reliability variance in the eight subscales (Ware, Kosinski, \& Keller, 1994). The physical functioning, role physical, and bodily pain subscales were found to be highly correlated with the physical component, thus contributing mostly to the physical component score (Ware, Kosinski, \& Keller, 1994). The mental health, role emotional, and social functioning subscales were highly correlated with the mental health component measure. The vitality, general health perceptions, and social functioning were correlated with both components. Thus, the physical functioning, role physical, bodily pain, and general health subscales compose the physical health summary component. The vitality, social functioning, role emotional, and mental health subscales compose the mental health summary component. Reliability estimates for the physical and mental health components were .80 (Ware et al., 1993) and .90 (Ware, Kosinski, \& Keller, 1994), respectively. Reliability estimates for the subscales were also reported to be at least .80 for all the subscales except for the social functioning subscale that showed to have a 
reliability of .76 (Ware et al., 1993). Test- retest estimates over a 2 week period showed correlation coefficients at least .80 on all dimensions (Ware et al., 2000). The SF-36v2 has been used to measure QOL in men with prostate cancer (Hegarty, Wallace, \& Comber, 2008; Mikhail et al., 2006; Shikanov et al., 2008). The Spanish SF-36 was validated with ay group of elderly Mexican Americans $(n=65)$. Internal consistency reliability estimates were .80 or above on all scales except social functioning $(r=.69)$ (Bennett \& Riegal, 2003).

Table 1

Major Study Variables

\begin{tabular}{lll}
\hline Core Measure & Brief Description & Author \\
\hline Demographics & Demographic and background data. & Ballout, S. (2013) \\
$\begin{array}{l}\text { Prostate cancer } \\
\text { treatment side } \\
\text { effects }\end{array}$ & $\begin{array}{l}\text { University of California Los Angeles } \\
\text { Prostate Cancer Index (UCLA PCI) } \\
\text { measures prostate cancer treatment side } \\
\text { effects: sexual, urinary, and bowel } \\
\text { functioning and bother. }\end{array}$ & Litwin et al., 1998 \\
Masculinity & $\begin{array}{l}\text { Conformity to Masculine Norms Inventory } \\
\text { (CMNI) measures the socially constructed } \\
\text { norms and the conformity of men to } \\
\text { masculine behaviors. }\end{array}$ & Mahalik et al., 2003 \\
Relationship with & $\begin{array}{l}\text { Dyadic Adjustment Scale (DAS) measures } \\
\text { relationship satisfaction. }\end{array}$ & Spanier, 1976 \\
Quartner & $\begin{array}{l}\text { Short Form Health Survey version 2.0 (SF- } \\
\text { 36v2) measures the overall health status, } \\
\text { functional status, and health related QOL. }\end{array}$ & Ware, Kosinski, \& \\
& $\begin{array}{l}\text { Dewey, 2000 } \\
\end{array}$ & \\
\hline
\end{tabular}




\section{Data Management}

Study packets were prepared by the PI to include an informed consent, questionnaires (i.e., demographic data form, UCLA PCI, CMNI, DAS, and SF36v2 instruments), and list of resources. The questionnaires were labeled with the participant's identification number on each page. The packets were prepared before data collection sessions with potential participants; however, the PI ensured completeness of the questionnaire and packet at each session.

A code book identifying codes for responses and missing values was created by the PI to facilitate the coding of the information obtained. The code book was completed prior to initiating of data entry. Data were entered into SPSS 18. Data were reviewed by the PI continuously to minimize data entry errors. Examination of frequencies, histograms, and descriptive statistics were used to identify data entry errors, missing data, and outliers for demographic data and all study instruments. Discrepancies were managed by referring to the questionnaires. Prior to initiation of data analysis, specific items were recoded as indicated by the instrument tutorial or as appropriate to conduct the proposed statistical analyses. Missing data were kept, and their corresponding cases were not deleted. Missing values were coded as ' 9 ' or ' 99 ' in SPSS. During analysis, listwise deletion was used for missing data (Mertler \& Vannatta, 2013). Outliers were identified and cases may be deleted in order not to skew the results. Outliers were identified in urinary functioning and were deleted. Details about the management will be detailed in next chapter. 


\section{Data Analysis}

Data analysis was conducted using SPSS 18.0. Each interval level variable was assessed for normality using graphical and statistical analyses (Mertler \& Vannatta, 2013). Linearity was also assessed as appropriate. Assuming normality and linearity are basic assumptions for the conduct of multivariate analysis (Mertler \& Vannatta, 2013).

Sample description. Frequency distributions, measures of central tendency (i.e., mean, median, mode), measures of variability (i.e., range, standard deviation), and inferential statistics were used to examine the demographic characteristics and major study variables.

Reliability of instruments. Internal consistency of the instruments (i.e., UCLAPCI, CMNI, DAS, SF36v2) were computed using Cronbach's alpha ( $r$ ) for the English and Spanish versions. Internal consistencies were compared with the published values of the original tools.

Research Question 1. Is there a relationship among age, ethnicity, sexual dysfunction, urinary incontinence, masculinity, and perception of the relationship with the partner, and quality of life in self identified heterosexual and married/partnered men diagnosed with prostate cancer?

Hypothesis 1. There is a relationship between age, ethnicity, sexual dysfunction, urinary incontinence, masculinity, and perception of the relationship with the partner, and quality of life in self identified heterosexual and married/partnered men diagnosed with prostate cancer.

Pearson Product Moment correlations $(r)$ were used to examine the relationships between age, urinary incontinence, sexual dysfunction masculinity, perceived 
relationship with partner, and quality of life. These variables are continuous measures, and therefore Pearson $r$ was the appropriate statistical test to identify relationships. Chi square analysis was conducted to examine the relationship between ethnicity and quality of life since ethnicity is a nominal level variable.

Research Question 2. What are the relative contributions of age, ethnicity, sexual dysfunction, urinary incontinence, masculinity, and perception of the relationship with the partner on men's quality of life in self identified heterosexual and married/partnered men diagnosed with prostate cancer?

Hypothesis 2. Age, ethnicity, sexual dysfunction, urinary incontinence, masculinity, and perception of the relationship with the partner will make independent and combined contributions to the quality of life of men diagnosed with prostate cancer.

Regression analyses was used to examine research question two to specify the independent and relative contributions of age, ethnicity, sexual dysfunction, urinary incontinence, masculinity, and perception of the relationship with the partner on QOL. Ethnicity was dummy coded (i.e., Hispanic and non-Hispanic) before being entered into the regression equation. This decision was made since the sample mirrored the population in Miami and therefore was predominantly Hispanic. Regression analysis is appropriate to explain the contribution of the five independent variables on the dependent variable (Mertler \& Vannatta, 2013). The correlational analyses used to answer the first research question provided information about multicollinearity and the degree of the relationships among the independent variables. The $F$ test was used to determine the strength of the relationships between the independent variables and the dependent 
variable. The regression coefficients $(\beta)$ were used to identify the contribution of the independent variables to explain the dependent variable (Mertler \& Vannatta, 2013). 


\section{CHAPTER IV}

\section{RESULTS}

The purpose of this study was to examine relative contributions of age, ethnicity, sexual dysfunction, urinary incontinence, masculinity, and perception of the relationship with the partner on the quality of life in men diagnosed with prostate cancer. English or Spanish speaking heterosexual and partnered men diagnosed with prostate cancer were recruited from four urology clinics and two support groups in Miami and Broward counties in Florida. Recruitment of participants was accomplished through close collaboration with the urology clinics and support groups. A total of 117 men completed the self administered study questionnaire that included a demographic data form, the University of California Los Angeles Prostate Cancer Index (UCLA PCI), Conformity to Masculine Norms Inventory (CMNI), Dyadic Adjustment Scale (DAS), and Quality of Life (Short Form Health Survey version 2.0 [SF36v2]) instruments.

\section{Pilot}

English and Spanish versions of the study instruments and informed consent were available for participants to choose their preferred language. The Conformity of Masculine Norms Inventory (CMNI) was not available in Spanish. Hence, it was translated by two independent Spanish speaking nursing students who were born in Cuba and Colombia, respectively, and lived in Miami for more than five years. Both translated versions were compared and a final Spanish CMNI version was agreed upon by an independent Spanish speaking experienced Cuban Nurse Practitioner. A third graduate nursing student back translated the Spanish CMNI to English. The back translation was compared to the original CMNI English version (Polit \& Beck, 2012). There were no 
major differences in original and back translated English versions of the CMNI. Accordingly, the Spanish version of the CMNI was administered as part of the study questionnaire. The informed consent was also translated from English to Spanish following the same procedure.

A pilot test was conducted to validate the comprehension and legibility of the Spanish versions of the instruments. The Hispanic population in Miami is predominantly Cuban, Venezuelan, Columbian, and other Caribbean and Latin American ethnicities; thus, it was deemed necessary to ensure understanding of the items of the questionnaires that have been validated with Hispanic populations who were primarily from Mexican or Puerto Rican origins. Another purpose of the pilot was to identify the duration of time needed to complete the study instruments.

A total of 10 Spanish speaking men (i.e., from Cuba, Columbia, Venezuela, and Dominican Republic) who met the inclusion criteria were recruited from the community. The pilot study participants read and answered the demographic form and study instruments. The pilot participants suggested adding "Black Caribbean" as one of the ethnicity options. The other demographic questions and study instruments were deemed understandable, clear, and culturally appropriate. There were no items or questions that were considered to be offensive or written in a manner that was thought to be culturally inappropriate. The 10 pilot participants took 20 to 35 minutes to complete the questionnaire. After completing the pilot, the suggestion of the pilot participants was considered and "Black Caribbean" was added as one of the ethnicity answer options in the English and Spanish versions of the questionnaire. Also, the average time needed to complete the questionnaire was added to the study material (i.e., flyer and cover letter) 
and informed consent. The average time needed to complete the questionnaire and the successful completion of the pilot phase was discussed during recruitment of research sites and study participants.

\section{Data Screening}

Prior to conducting the analysis, the data were screened for missing values and outliers, and tests were conducted to assess normality, linearity, and homoscedasticity. Data screening and exploratory data analysis aimed at ensuring the validity of the multivariate analyses to be conducted. Frequency distributions and descriptive statistics for all variables were conducted to make sure that values did not lie outside the range of possible values in the case of continuous variables or coded values in the case of categorical variables. The scores that fell outside the range of legitimate values were identified as missing values (Van den Broeck, Cunningham, Eeckels, \& Herbst, 2005). Missing data were kept, and their corresponding values were coded as "9" or "99", as appropriate. During analysis, listwise deletion was used (Howell, 2009). The data were assessed for presence of outliers. There were no major outliers that required deletion of cases.

Dummy coding was used for ethnicity; data were recoded into 0 (nonHispanic) and 1 (Hispanic) to conduct analyses to answer the research questions of the study. White/Caucasian, African American, and Black Caribbean ethnicities were considered to be nonHispanic. White Hispanic and Black Hispanic were considered to be Hispanic. Using the scoring manuals of the instruments, variables were recoded and total values of the urinary functioning, sexual functioning, CMNI, DAS, and QOL were computed. 
The assumptions of normality, linearity and homoscedasticity of the major study variables (i.e., age, dummy coded ethnicity, urinary functioning, sexual functioning, CMNI, DAS, and QOL) were assessed (Mertler \& Vannatta, 2013). Normality was explored by examination of the histogram of each variable. Skewness and kurtosis coefficients for the major study variables (i.e., age, ethnicity, sexual function, urinary function, masculinity, relationship with partner, and QOL) were also examined. Age, ethnicity, CMNI, DAS, and QOL showed negative skewness and kurtosis. Skewness of all interval level major study variables lied within -1 and +1 ; where values ranged between -.37 for the CMNI and +.90 for urinary functioning. Kurtosis for age, CMNI, DAS, DAS, and QOL lied with -1 and +1 . However, kurtosis values for urinary functioning and sexual functioning were 1.90 and 1.96, respectively. Examination of the means and standard deviations $(S D)$ of urinary functioning and sexual functioning scores showed that the $S D$ was relatively high (urinary functioning $M=31.57, S D=14.46$; sexual functioning $M=32.95, S D=12.38)$. Scores for urinary functioning $(n=116)$ ranged between 5 and 82; 7 cases had scores below 10, and 4 cases had scores above 6 . Scores for sexual functioning $(n=117)$ ranged between 0 and 69 , and 6 cases were above 60. Examination of the $Q-Q$ Plots of the variables confirmed the results of kurtosis. Therefore, the cases with extreme values were excluded and counted as missing values. The recoded variables were identified as urinary functioning 2 and sexual functioning 2 . Urinary functioning $2(n=105, M=31.58, S D=10.61)$ had a positive kurtosis of .17 in addition to slight positive skewness. Sexual functioning $2(n=111, M=31.04, S D=$ 9.48) had kurtosis of .64 and maintained slight negative skewness. Urinary functioning 2 and sexual functioning 2 were used for the analysis of the data from this point forward 
and was referenced as urinary functioning and sexual functioning. Normal Q-Q plots were observed for the other variables.

Linearity was assessed by observing the scatterplots of the independent and dependent variables. Scatterplots of age, ethnicity, urinary functioning, sexual functioning, CMNI, DAS, and QOL were inspected (Mertler \& Vannatta, 2013). The scatterplots showed nonelliptical shapes for all variable combinations. To further investigate linearity, the standardized residuals were compared with the predicted values of the dependent variable (i.e., QOL). The scatterplot of the standardized predicted values by standardized residuals and the normal $P-P$ Plot was examined. Hence, the assumptions of normality and linearity were met. Homoscedasticity or homogeneity of variances were tested using Levene's Test as part of the statistical analyses discussed below.

\section{Description of the Sample}

The sample ranged in age from 52 to 85 years with a mean age of $67.47(S D=$ 8.42) years (see Table 2). More than half the sample identified themselves to be of Hispanic origin $(n=63,54.3 \%)$. The ethnic distribution mirrored that of South Florida where the most men identified themselves as White Hispanic $(n=39,33.6 \%)$, Black Hispanic $(n=24,20.7 \%)$, and Black Caribbean $(n=19,16.4 \%)$. The majority of men in the sample were born in the United States $(n=34,29.8 \%)$, Cuba $(n=23,20.2 \%)$, or Haiti $(n=12,10.5 \%)$. However, the majority identified that their family was originally from Cuba $(n=24,25 \%)$, Haiti $(n=12,12.5 \%)$, or the United States $(n=11,11.5 \%)$.

The inclusion criteria of the study required men to be partnered (i.e., married or cohabitating). More than half the sample were married $(n=61,52.1 \%)$ or living with 
their partner $(n=45,38.5 \%)$. The majority of the men were involved in the current relationship for more than one year $(n=92,79.3 \%)$. Few couples chose not to live together $(n=11,9.4 \%)$. Most men had three $(n=43,37.1 \%)$ or two children $(n=36$, $31 \%)$.

Table 2

Demographic Characteristics of the Sample $(N=117)$

\begin{tabular}{cccccc}
\hline Variables & Total Sample & Hispanic & NonHispanic & Statistic & $p$ \\
$N(\%)$ & $n=63$ & $n=53$ & & value \\
& $n(\%)$ & $n(\%)$ & &
\end{tabular}

Age $[M(S D)]$

$67.57(8.42)$

$66.60(8.56)$

$68.55(8.20)$

$t=1.24$

.22

Ethnicity

$\begin{array}{ll}\text { White/ Caucasian } & 17(14.7 \%) \\ \text { White Hispanic } & 39(33.6 \%) \\ \text { African American } & 17(14.7 \%) \\ \text { Black Hispanic } & 24(20.7 \%) \\ \text { Black Caribbean } & 19(16.4 \%)\end{array}$

Country of Birth

United States

$34(29.8 \%)$

Cuba

$23(20.2 \%)$

Haiti

$12(10.5 \%)$

Columbia

$10(8.8 \%)$

Venezuela

9 ( $7.9 \%)$

Others

$26(22.9 \%)$

Relationship Status

$\begin{array}{lccrrr}\text { Living with partner } & 45(38.5 \%) & 28(44.4 \%) & 17(32.1 \%) & & \\ \text { Married } & 61(52.1 \%) & 31(49.2 \%) & 29(54.7 \%) & \chi^{2}= & .26 \\ \text { Not living with } & 1(9.4 \%) & 4(6.3 \%) & 7(13.2 \%) & 2.73 & \end{array}$

Number of children

\begin{tabular}{lccccc}
1 & $7(6.0 \%)$ & $3(4.8 \%)$ & $4(7.5 \%)$ & & \\
2 & $36(31.0 \%)$ & $16(25.8 \%)$ & $20(37.7 \%)$ & $\chi^{2}=$ & .54 \\
3 & $43(37.1 \%)$ & $25(40.3 \%)$ & $17(32.1 \%)$ & 3.10 & \\
4 & $23(19.8 \%)$ & $13(21.0 \%)$ & $10(18.9 \%)$ & & \\
5 or more & $7(6.0 \%)$ & $5(8.1 \%)$ & $2(3.8 \%)$ & & \\
\hline
\end{tabular}


Table 2 (Continued)

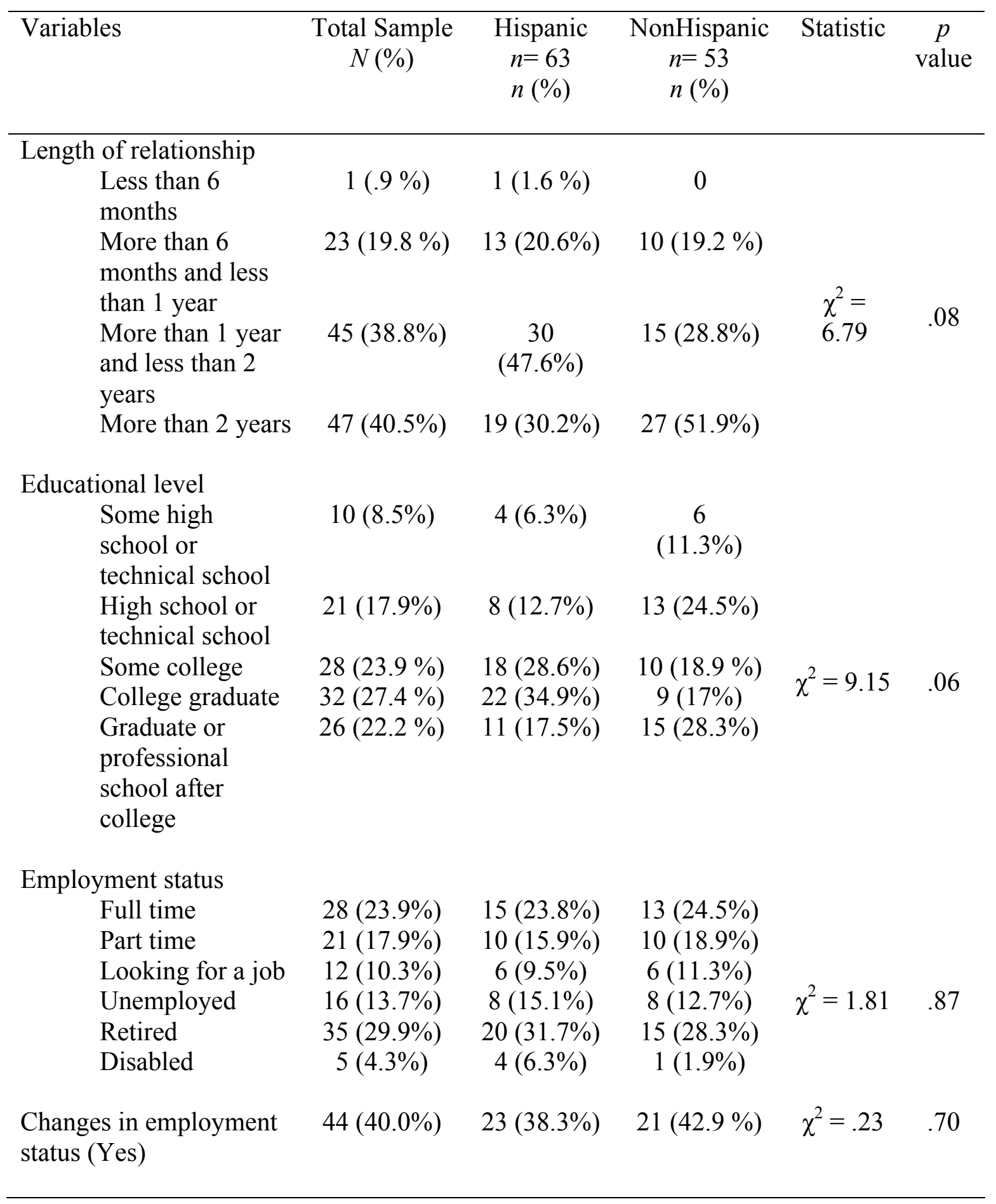


Table 2 (Continued)

\begin{tabular}{|c|c|c|c|c|c|}
\hline Variables & $\begin{array}{c}\text { Total Sample } \\
N(\%)\end{array}$ & $\begin{array}{c}\text { Hispanic } \\
n=63 \\
n(\%)\end{array}$ & $\begin{array}{c}\text { NonHispanic } \\
n=53 \\
n(\%)\end{array}$ & Statistic & $\begin{array}{c}p \\
\text { value }\end{array}$ \\
\hline $\begin{array}{l}\text { Annual income } \\
\text { None } \\
\$ 15,000 \text { or less } \\
\$ 15,001 \text { to } \\
\$ 25,000 \\
\$ 25,001 \text { to } \\
\$ 50,000 \\
\$ 50,001 \text { to } \\
\$ 80,000 \\
\$ 80,001 \text { to } \\
\$ 100,000\end{array}$ & $\begin{array}{l}14(12.1 \%) \\
21(18.1 \%) \\
11(9.5 \%) \\
27(23.3 \%) \\
24(20.7 \%) \\
19(16.4 \%)\end{array}$ & $\begin{array}{c}10(15.9 \%) \\
14(22.2 \%) \\
7(11.1 \%) \\
14(22.2 \%) \\
11(17.5 \%) \\
7(11.1 \%)\end{array}$ & $\begin{array}{c}4(7.7 \%) \\
7(13.5 \%) \\
4(7.7 \%) \\
12(23.1 \%) \\
13(25 \%) \\
12(23.1 \%)\end{array}$ & $\chi^{2}=6.37$ & .27 \\
\hline
\end{tabular}

Men in this sample were generally educated and around half the sample reported being at least a college graduate $(n=58,49.6 \%)$. Only $41.8 \%(n=49)$ of participants reported to be employed either part time or full time. The majority were retired ( $n=35$, $29.9 \%$ ), unemployed ( $n=16,13.7 \%)$, or looking for a job $(n=12,10.3 \%)$. Around $60 \%$ ( $n=70)$ of the participants reported their annual income to be more than $\$ 25,000$ but less than $\$ 100,000$. After the diagnosis of prostate cancer only $40 \%(n=44)$ had to change or leave their job.

Data were collected from four urology clinics. Participants from the four research sites were compared on demographics such as age, ethnicity, relationship status, and presence of comorbidities. Analysis of variance (ANOVA) showed that there were no differences in age among the six clinic sites. Chi-square analysis showed that there were no differences among the clinics in ethnicity, relationship status, and presence of comorbidities. 
Prostate cancer disease status and presence of comorbidities was investigated (see Table 3). Most men in this sample complained of asthma, emphesyma, or breathing problems $(n=15,15.3 \%)$ and circulation problems in the lower extremities $(n=15$, $15.3 \%)$. Many men were smokers or had current or past smoking related problems ( $n=$ $14,14.3 \%)$. Others endured other conditions such as stroke $(n=11,11.2 \%)$, amputation $(n=10,10.2 \%)$, diabetes $(n=8,8.2 \%)$, kidney disease $(n=6,5.1 \%)$, heart problems $(n=$ $6,5.1 \%$ ), or others. The majority of men participating in this study were diagnosed with prostate cancer more than one year $(n=70,59.9 \%)$ ago. The PSA levels ranged between 0.12 and $11.20(M=3.260, S D=2.01)$. Men in this study were mostly diagnosed with stages II $(n=44,38.3 \%)$ and III $(n=46,40 \%)$. They were treated with different options such as radiation therapy $(n=29,25.4 \%)$, hormonal therapy $(n=21,17.9 \%)$, watchful waiting $(n=21,17.9 \%)$, and surgery $(n=16,13.7 \%)$. Some men also had more than one form of treatment $(n=22,18.8 \%)$. 
Table 3

Disease Status $(N=117)$

\begin{tabular}{|c|c|c|c|c|c|}
\hline Variables & $\begin{array}{l}\text { Total } \\
\text { Sample } \\
N(\%)\end{array}$ & $\begin{array}{c}\text { Hispanic } \\
n=63 \\
n(\%)\end{array}$ & $\begin{array}{c}\text { NonHispanic } \\
n=53 \\
n(\%) \\
\end{array}$ & Statistic & $\begin{array}{c}p \\
\text { value }\end{array}$ \\
\hline \multicolumn{6}{|l|}{ Comorbidities } \\
\hline $\begin{array}{l}\text { Asthma, } \\
\text { emphesyma, } \\
\text { breathing } \\
\text { problems }\end{array}$ & $15(15.3 \%)$ & $\begin{array}{c}13 \\
(23.2 \%)\end{array}$ & $2(4.9 \%)$ & & \\
\hline $\begin{array}{l}\text { Circulation } \\
\text { problems in legs } \\
\text { or feet }\end{array}$ & $15(15.3 \%)$ & $7(12.5 \%)$ & $8(19.5 \%)$ & & \\
\hline $\begin{array}{l}\text { Current or past } \\
\text { smoking } \\
\text { problems }\end{array}$ & $14(14.3 \%)$ & $\begin{array}{c}6 \\
(10.7 \%)\end{array}$ & $8(19.5 \%)$ & $\chi^{2}=14.0$ & .23 \\
\hline Stroke & $11(11.2 \%)$ & $4(7.1 \%)$ & $7(17.1 \%)$ & & \\
\hline Amputation & $10(10.2 \%)$ & $\begin{array}{c}7 \\
(12.5 \%)\end{array}$ & $3(7.3 \%)$ & & \\
\hline Diabetes & $8(8.2 \%)$ & $4(7.1 \%)$ & $3(7.9 \%)$ & & \\
\hline Kidney disease & $6(5.1 \%)$ & $2(3.6 \%)$ & $4(9.8 \%)$ & & \\
\hline Heart problems & $6(61 \%)$ & $4(7.1 \%)$ & $2(4.9 \%)$ & & \\
\hline Others & $13(13.4 \%)$ & $\begin{array}{c}9 \\
(16.2 \%)\end{array}$ & $4(9.8 \%)$ & & \\
\hline \multicolumn{6}{|l|}{$\begin{array}{l}\text { Duration of prostate } \\
\text { cancer }\end{array}$} \\
\hline $\begin{array}{l}\text { Less than } 1 \\
\text { month }\end{array}$ & $1(0.9 \%)$ & $0(0.0 \%)$ & $1(1.9 \%)$ & & \\
\hline 1 to 3 months & $30(25.6 \%)$ & $17(27 \%)$ & $13(24.5 \%)$ & & \\
\hline 3 to 6 months & $4(3.4 \%)$ & $2(3.2 \%)$ & $2(3.8 \%)$ & & \\
\hline $\begin{array}{l}6 \text { months to } 1 \\
\text { year }\end{array}$ & $12(10.3 \%)$ & $7(11.1 \%)$ & $5(9.4 \%)$ & $\chi^{2}=1.38$ & .93 \\
\hline 1 to 2 years & $32(27.4 \%)$ & $17(27 \%)$ & $15(28.3 \%)$ & & \\
\hline $\begin{array}{l}\text { More than } 2 \\
\text { years }\end{array}$ & $38(32.5 \%)$ & $\begin{array}{c}20 \\
(31.7 \%)\end{array}$ & $17(32.1 \%)$ & & \\
\hline
\end{tabular}


Table 3 (Continued)

\begin{tabular}{|c|c|c|c|c|c|}
\hline Variables & $\begin{array}{c}\text { Total } \\
\text { Sample } \\
N(\%)\end{array}$ & $\begin{array}{c}\text { Hispanic } \\
n=63 \\
n(\%)\end{array}$ & $\begin{array}{c}\text { NonHispanic } \\
n=53 \\
n(\%)\end{array}$ & Statistic & $p$ value \\
\hline \multicolumn{6}{|l|}{ Prostate cancer stage } \\
\hline Stage I & $21(18.3 \%)$ & $14(22.6 \%)$ & $7(13.5 \%)$ & $\chi^{2}=1,67$ & .64 \\
\hline Stage II & $44(38.3 \%)$ & $22(35.5 \%)$ & $22(42.3 \%)$ & & \\
\hline Stage III & $46(40.0 \%)$ & $24(38.7 \%)$ & $21(40.4 \%)$ & & \\
\hline Stage IV & $4(3.5 \%)$ & $2(3.2 \%)$ & $2(3.8 \%)$ & & \\
\hline $\begin{array}{l}\text { Last PSA level }[M \\
(S D)]\end{array}$ & $3.26(2.01)$ & $3.0(1.90)$ & $3.57(2.15)$ & $t=1.33$ & .19 \\
\hline \multicolumn{6}{|l|}{$\begin{array}{l}\text { Prostate cancer stag } \\
\text { Prostate cancer } \\
\text { treatment }\end{array}$} \\
\hline $\begin{array}{l}\text { Radiation } \\
\text { therapy }\end{array}$ & $29(25.4 \%)$ & $\begin{array}{c}16 \\
(25.4 \%)\end{array}$ & $12(24.0 \%)$ & & \\
\hline $\begin{array}{l}\text { Hormone } \\
\text { therapy }\end{array}$ & $23(19.7 \%)$ & $\begin{array}{c}13 \\
(20.6 \%)\end{array}$ & $10(20 \%)$ & & \\
\hline $\begin{array}{l}\text { Watchful } \\
\text { waiting }\end{array}$ & $21(17.9 \%)$ & $\begin{array}{c}11 \\
(17.5 \%)\end{array}$ & $10(20 \%)$ & $\chi^{2}=2.80$ & .73 \\
\hline Prostate surgery & $16(13.7 \%)$ & $9(14.3 \%)$ & $7(14 \%)$ & & \\
\hline $\begin{array}{l}\text { More than } 1 \\
\text { treatment option }\end{array}$ & $22(18.8 \%)$ & $\begin{array}{c}11 \\
(17.5 \%)\end{array}$ & $11(22 \%)$ & & \\
\hline Other & $3(2.6 \%)$ & $3(4.8 \%)$ & $0(0.0 \%)$ & & \\
\hline
\end{tabular}


Table 4

Participation of Men in Treatment Decision $(N=117)$

\begin{tabular}{|c|c|c|c|c|c|}
\hline Variables & $\begin{array}{c}\text { Total } \\
\text { Sample } \\
N(\%)\end{array}$ & $\begin{array}{l}\text { Hispanic } \\
n=63 \\
n(\%)\end{array}$ & $\begin{array}{c}\text { NonHispanic } \\
n=53 \\
n(\%)\end{array}$ & Statistic & $p$ value \\
\hline $\begin{array}{l}\text { Participated in } \\
\text { treatment decision }\end{array}$ & $\begin{array}{c}65 \\
(55.6 \%)\end{array}$ & $\begin{array}{c}30 \\
(51.7 \%)\end{array}$ & $\begin{array}{c}34 \\
(69.4 \%)\end{array}$ & $\chi^{2}=3.44$ & .06 \\
\hline $\begin{array}{l}\text { Preferences taken into } \\
\text { treatment decision }\end{array}$ & $\begin{array}{c}52 \\
(44.4 \%)\end{array}$ & $\begin{array}{c}27 \\
(49.1 \%)\end{array}$ & $\begin{array}{c}25 \\
(58.1 \%)\end{array}$ & $\chi^{2}=1.02$ & .37 \\
\hline $\begin{array}{l}\text { Concerns discussed } \\
\text { prior to making } \\
\text { treatment decisions }\end{array}$ & $\begin{array}{c}62 \\
(53 \%)\end{array}$ & $\begin{array}{c}31 \\
(54.4 \%)\end{array}$ & $\begin{array}{c}31 \\
(60.8 \%)\end{array}$ & $\chi^{2}=.45$ & .50 \\
\hline
\end{tabular}

A $t$-test showed that there was no difference in mean PSA levels among Hispanic and nonHispanic participants. Chi-square analysis also showed no significant differences in comorbidities, duration of prostate cancer, stage of prostate cancer and treatment option among Hispanic and nonHispanic participants.

Around two thirds of the participants participated in choosing the treatment option for prostate cancer (see Table 4). The preferences of the treatment were taken into consideration in less than half the sample $(n=52,44.4 \%)$. The concerns about the prostate cancer treatment were discussed with $62(53.0 \%)$ participants. Chi-square analysis showed that there were no differences in the participation in the treatment decision between Hispanic and nonHispanic men in this sample.

Sexual problems before the diagnosis of prostate cancer and after the diagnosis of the disease as well as sexual enhancement regimens used were examined. Less than $50 \%$ 
of the sample answered the question related to having sexual problems prior to the diagnosis of prostate cancer. Out of those who answered, 31 (44.9\%) reported having sexual problems prior to the diagnosis of prostate cancer. After the diagnosis of prostate cancer and during recent times, the majority of men reported that their sexual desire $(n=$ $88,75.2 \%)$, ability to have an erection $(n=89,79.5 \%)$, and ability to reach an orgasm ( $n$ $=92,78.6 \%)$ were poor or very poor on a five point Likert scale. Only 9 men $(7.7 \%)$ had an erection that was firm enough for intercourse. Also, only 29 men (24.8\%) reported having an erection whenever they wanted. Regarding the use of sexual enhancement regimens, men used medications or pills $(n=37,34.9 \%)$, injections $(n=34,32.1 \%)$, vacuum erection device $(n=15,14.2 \%)$, penile implants $(n=10,9.4 \%)$, and others $(n=$ 10, 9.4\%). Chi-square analysis demonstrated that there were no significant differences in the use of sexual enhancement regimens between Hispanic and nonHispanic men. 
Table 5

Sexual Issues Related to Prostate Cancer $(N=117)$

\begin{tabular}{|c|c|c|c|c|c|}
\hline Variables & $\begin{array}{c}\text { Total } \\
\text { Sample } \\
N(\%)\end{array}$ & $\begin{array}{c}\text { Hispanic } \\
n=63 \\
n(\%)\end{array}$ & $\begin{array}{c}\text { NonHispanic } \\
n=53 \\
n(\%)\end{array}$ & Statistic & $p$ value \\
\hline $\begin{array}{l}\text { Sexual problems before } \\
\text { prostate cancer }\end{array}$ & $31(44.9 \%)$ & $\begin{array}{c}15 \\
(40.5 \%)\end{array}$ & $16(50.0 \%)$ & $\chi^{2}=.62$ & .43 \\
\hline \multicolumn{6}{|l|}{$\begin{array}{l}\text { Use of sexual } \\
\text { enhancement regimens }\end{array}$} \\
\hline $\begin{array}{l}\text { Medication or } \\
\text { pills }\end{array}$ & $37(34.9 \%)$ & $\begin{array}{c}22 \\
(38.6 \%)\end{array}$ & $15(31.3 \%)$ & \multirow{6}{*}{$\chi^{2}=9.81$} & \multirow{6}{*}{.04} \\
\hline $\begin{array}{l}\text { Injections } \\
\text { (intracavernous }\end{array}$ & $34(32.1 \%)$ & $\begin{array}{c}16 \\
(28.1 \%)\end{array}$ & $17(35.4 \%)$ & & \\
\hline injections) & & & & & \\
\hline $\begin{array}{l}\text { Vacuum erection } \\
\text { device }\end{array}$ & $15(12.8 \%)$ & $8(14 \%)$ & $7(14.6 \%)$ & & \\
\hline Penile implants & $10(8.5 \%)$ & $3(5.3 \%)$ & $7(14.6 \%)$ & & \\
\hline Others & $10(8.5 \%)$ & $8(14 \%)$ & $2(4.2 \%)$ & & \\
\hline
\end{tabular}

Reliability of Study Instruments

Reliability analysis was computed for the study instruments: University of California Los Angeles Prostate Cancer Index (UCLA PCI), Conformity to Masculine Norms Inventory (CMNI), Dyadic Adjustment Scale (DAS), and Quality of Life (Short Form Health Survey version 2.0 [SF36v2]). Cronbach's alpha for the UCLA PCI, DAS, and SF36v2 were $.53, .76$, and .85 respectively. Theta was calculated to study the reliability of CMNI and was .84. A $t$-test was conducted to identify differences among the means of the major study instruments. Results showed that there were no significant differences in the means of UCLA PCI, CMNI, DAS, and SF36v2 between Hispanic and nonHispanic participants. 
The instruments were administered in English and Spanish versions. A total of 62 men (53\%) answered the questionnaire in Spanish. A $t$-test was conducted to examine differences in the means of UCLA PCI, DAS, and SF36v2 between English and Spanish versions of the instruments. There were no significant differences in the means of the English and Spanish UCLA PCI, DAS, and SF36v2.

\section{Description of Major Study Variables}

The study included five independent variables and one dependent variable. The variables were measured using valid and reliable instruments. The independent variables were age, ethnicity, sexual dysfunction, urinary incontinence, masculinity, and relationship with the partner. The dependent variable was quality of life of men with prostate cancer. Age and ethnicity have been described as part of the description of the sample. This section will focus on sexual dysfunction, urinary incontinence, masculinity, relationship with the partner, and quality of life (see Table 6).

Table 6

Description of Major Study Variables $(N=117)$

\begin{tabular}{lc}
\hline Variables & $M(S D)$ \\
\hline Urinary Functioning & $31.58(10.61)$ \\
Sexual Functioning & $31.04(9.48)$ \\
Conformity to Masculine Norms Inventory (CMNI) & $30.95(7.194)$ \\
Dyadic Adjustment Scale (DAS) & $105.93(10.46)$ \\
Quality of life (QOL) & $60.58(9.50)$ \\
\hline
\end{tabular}




\section{Sexual Dysfunction}

Sexual dysfunction was measured by the sexual function subscale of the UCLA PCI. The majority of men described their sexual desire $(n=78,77.3 \%)$, ability to have an erection $(n=93,79.5 \%)$, and ability to have an orgasm $(n=92,78.6 \%)$ as "poor" or "very poor". Fifty two men $(44.5 \%)$ rated the quality of their erection to be completely absent or not firm enough for sexual activity. Only $9(7.7 \%)$ reported a quality of erection that was "firm enough for intercourse". Twenty nine $(24.8 \%)$ said that they had an erection whenever they wanted. Then again, only $5(4.3 \%)$ were "often" (i.e., more than half the time) awakened with an erection at night or in the morning. Items inquiring about sexual behaviors during the last 4 weeks showed that 53 participants $(48.3 \%)$ had vaginal or anal intercourse. Most men identified their ability to function sexually $(n=92$, $78.6 \%)$ during the last 4 weeks as "poor" and "very poor". Over two fifths $(n=42,37 \%)$ and $(n=41,36.3 \%)$ of men who participated in the study classified their sexual functioning as a "big problem" and "moderate problem", respectively, in the past 4 weeks. The mean score of the sexual functioning subscale of the UCLA PCI was 31.04 $(S D=9.48)$

Pearson $r$ correlation analysis showed no relationship among sexual function, age, and last PSA level. A $t$ - test analysis demonstrated that there were no differences in sexual functioning between Hispanic and nonHispanic participants. Analysis of Variance (ANOVA) showed no differences in sexual functioning and relationship status, presence of comorbidities, duration of prostate cancer, stage of prostate cancer, treatment option, presence of sexual problems before acquiring prostate cancer, and use of sexual 
enhancement methods (i.e., medications or pills, injections, vacuum device, penile implants, or others).

\section{Urinary Dysfunction}

During the past four weeks, 59 (50\%) of the participants had urinary leakage every day with 57 (48.7\%), 49 (91.9\%), and 11 (9.4\%) reporting occasional dribbling, frequent dribbling, or no control what so ever, respectively. The majority of men $(n=98$, $84.5 \%$ ) used one or more pads for urinary leakage in the past four weeks. Eighty six (73.5\%) considered their dribbling urine or wetting to be a moderate or big problem. Similarly, $91(77.8 \%)$ identified their urinary leakage interfering with sexual activity as a moderate and big problem. However, only 57 (48.7\%) considered their urinary function to be a moderate to big problem during the last 4 weeks. The mean score of the urinary functioning subscale of the UCLA PCI was $31.58(S D=10.61)$.

Pearson $r$ correlations showed no significant relationships between urinary functioning, age, and the last PSA level. There were no significant differences in urinary functioning between Hispanic and nonHispanic men in this study. There was a significant difference in urinary functioning and duration since the diagnosis of prostate cancer $(F(4,100)=3.23, p=.015)$. Tamhane post hoc analysis showed that the significant difference was between those diagnosed 6 months to 1 year ago and those diagnosed more than 2 years ago (Mertler \& Vannatta, 2013). Men diagnosed more than 2 years ago had more urinary functioning issues. There was also a significant difference in urinary functioning among the different prostate cancer stages $(F(3,10094)=4.08, p=$ .009). Tamhane post hoc analysis revealed that there were significant differences between stage II and Stage III where the former had more urinary functioning issues. 
There was also a difference between stages III and IV, and the latter group had the most urinary functioning issues. There were also significant differences in urinary functioning among the different treatment options $(F(5,97)=3.18, p=.01)$. Post hoc analysis showed that those undergoing hormonal therapy complained of urinary functioning issues more than those who had prostate surgery. There were no significant differences in urinary functioning and relationship status, comorbidities, presence of sexual problems before the diagnosis of prostate cancer, and the use of different sexual enhancement regimens.

\section{Masculinity}

The CMNI scores ranged from 16 to 44 . The mean score of the conformity to masculine norms was $30.95(S D=7.19)$. Correlation analysis showed that there was no significant relationship between CMNI and age and last PSA level. A $t$-test showed no difference in the conformity to masculine norms between Hispanic and nonHispanic men in this study. One way ANOVA showed no significant differences in CMNI scores among relationship statuses, number of years in the relationship, educational status, duration of acquiring prostate cancer, stage of prostate cancer, use of sexual enhancement regimens, and having sexual problems before the diagnosis of prostate cancer.

\section{Relationship with the Partner}

The total DAS scores ranged between 76 and 124. The mean total DAS score was $105.93(S D=10.46)$. Examining the DAS subscales, participants in this study scored highest on the consensus subscale $(M=48.95, S D=6.79)$ followed by satisfaction $(M=32.93, S D=4.41)$, cohesion $(M=15.81, S D=4.20)$, and affectional expression $(M=8.18, S D=2.39)$ subscales. 
A $t$ - test showed no significant differences in DAS scores between Hispanic and nonHispanic men. Correlation analysis demonstrated no relationship between total DAS scores, age, and last PSA levels. One way ANOVA showed no significant differences in DAS scores among relationship statuses, number of years in the relationship, educational status, duration of acquiring prostate cancer, stage of prostate cancer, and having sexual problems before the diagnosis of prostate cancer. One way ANOVA showed a significant difference in mean DAS scores among the use of different sexual enhancement regimens $(F(4,94)=2.55, p=.044)$. However, post hoc analysis did not show significant differences among the different groups of sexual enhancement regimens.

\section{Quality of Life}

Over two thirds $(n=75,64 \%)$ of men rated their general health as "good" or "very good". Only $30(25.6 \%)$ thought that their general health was worse than one year ago. Most men described their health as limiting a little vigorous activities $(n=65$, $55.6 \%)$, moderate activity $(n=51,43.6 \%)$, lifting or groceries $(n=54,46.2 \%)$, climbing several flights of stairs $(n=55,47 \%)$, and walking one mile $(n=55,47 \%)$. Men had no health related limitations related to walking several hundred yards $(n=61,52.1 \%)$, bathing or dressing $(n=54,47 \%)$. The QOL scores ranged between 36 and 78 and had a mean of $60.58(S D=9.50)$. The subscale of QOL that demonstrated the highest mean was role limitations due to physical health $(M=72.01, S D=22.02)$, and the lowest mean was in the mental health subscale $(M=51.62, S D=10.04)$.

Pearson $r$ correlations showed no significant relationship between QOL scores, age, and PSA levels. There were no significant differences in QOL scores among Hispanic and nonHispanic men in this study. One way ANOVA showed no significant 
differences in QOL scores among relationship status, duration of the relationship, educational status, duration of prostate cancer, stage of prostate cancer, and treatment option. One way ANOVA also showed significant difference in QOL scores between those who had sexual problems before the diagnosis of prostate cancer and those who did $\operatorname{not}(F(1,66)=3.83, p=.05)$. Those who did not have sexual problems before the diagnosis of prostate cancer had higher QOL scores than those who did. There were differences in QOL scores among those who use different sexual enhancement regimens $(F(4,99)=3.55, p=.009)$. Post hoc analysis showed that those taking medications or pills or using intracavernous injections had significantly higher QOL scores than those with penile implants.

Correlational analysis was conducted between the QOL subscales, urinary function, and sexual function. Findings showed that there was a significant negative relationship between urinary functioning and the social functioning subscale $(r=-.24, p=$ $.01)$. There was also a significant positive correlation between sexual functioning and bodily pain $(r=.20, p=.03)$ and a negative correlation between sexual functioning and general health subscale $(r=-.27, p=.005)$. Physical health scores were positively correlated with vitality $(r=.20, p=.03)$ and negatively correlated with bodily pain $(r=$ $.38, p<.001)$. The role limitations due to physical health subscale was positively correlated with role limitations due to emotions $(r=.43, p<.001)$, mental health $(r=.25$, $p=.006)$, social functioning $(r=.20, p=.03)$, and bodily pain $(r=.36, p<.001)$. There was a significant positive correlation between role limitations due to emotions and vitality $(r=.40, p<.001)$, mental health $(r=.31, p=.01)$, and bodily pain $(r=.19, p=$ $.04)$ subscales. Vitality was significantly associated with bodily pain $(r=.34, p<.001)$, 
social functioning $(r=.28, p=.003)$, and mental health $(r=.36, p<.001)$. Social functioning was also correlated with mental health $(r=.39, p<.001)$.

A physical health component and a mental health component of QOL were also computed. The physical health component included physical functioning, role limitations due to physical health, bodily pain, and general health. The mental health component was composed of the vitality, social functioning, role limitations due to emotions, and mental health subscales. Pearson $r$ correlations were computed to examine the relationship between the physical and mental health components of QOL, urinary function, and sexual function. The findings demonstrated that there was a significant positive relationship between both components of QOL $(r=.51, p<.001)$.

\section{Relationship Between Major Study Variables}

RQ1: Are there relationships between age, ethnicity, sexual dysfunction, urinary incontinence, masculinity, and perception of the relationship with the partner, and quality of life?

H1: There is a relationship between age, ethnicity, sexual dysfunction, urinary incontinence, masculinity, and perception of the relationship with the partner, and quality of life.

Pearson $r$ correlation was computed to examine the relationships between age, ethnicity, sexual dysfunction, urinary incontinence, masculinity, and perception of the relationship with the partner, and quality of life (see Table 7). The dummy coded ethnicity variable was used in the correlation analysis. Age and ethnicity were not associated with any of the major study variables. There was a moderate significant negative relationship between urinary and sexual functioning. There was a significant 
strong negative relationship between partner relationship and masculinity. There was also a weak negative relationship between DAS and QOL (see Table 7).

Table 7

Relationships Among Age, Ethnicity, Sexual Dysfunction, Urinary Incontinence, Masculinity, and Perception of The Relationship with the Partner, and Quality of Life

\begin{tabular}{|c|c|c|c|c|c|c|c|}
\hline Variables & 1 & 2 & 3 & 4 & 5 & 6 & 7 \\
\hline Age & 1 & & & & & & \\
\hline Ethnicity & & 1 & & & & & \\
\hline $\begin{array}{l}\text { Sexual } \\
\text { Functioning }\end{array}$ & & & 1 & & & & \\
\hline $\begin{array}{l}\text { Urinary } \\
\text { Functioning }\end{array}$ & & & $-.30^{* *}$ & 1 & & & \\
\hline Masculinity & & & & & 1 & & \\
\hline DAS & & & & & $-.41^{* * *}$ & 1 & \\
\hline QOL & & & & & & $-.21^{*}$ & 1 \\
\hline
\end{tabular}

\section{Contributions to QOL}

RQ2: What are the relative contribution of age, ethnicity, sexual dysfunction, urinary incontinence, masculinity, and perception of the relationship with the partner with men's quality of life? 
H2: Age, ethnicity, sexual dysfunction, urinary incontinence, masculinity, and perception of the relationship with the partner independently and combined contribute to the quality of life of men diagnosed with prostate cancer.

Hierarchal multiple regression was conducted to determine the relative contribution of age, ethnicity, sexual dysfunction, urinary incontinence, masculinity, and perception of the relationship with the partner with men's quality of life. The dummy coded ethnicity variable was used in the regression. The assumptions of regression were tested prior to running the regression analysis. As discussed in the data screening section earlier, presence of outliers were examined and corrected. Exploring multivariate outliers, Mahalanobis Distance was computed, and outliers were examined using chisquare critical value at $p<.001$ (Mertler \& Vannatta, 2013). There were no cases that exceeded the chi-square critical value. Univariate normality of the major study variables was assessed using histograms and $Q-Q$ plots as described in the data screening section above. Additionally, Kolomogorov-Smirniv test was not significant; hence the assumption of normality was met (Mertler \& Vannatta, 2013). Multivariate normality and linearity was examined using scatterplot. Multicollinearity was examined in the previous section by examining the correlation between the independent variables. Significant correlations were of moderate strength and therefore did not cause a multicollinearity threat (Mertler \& Vannatta, 2013).

Examining the correlations between the independent variables and the dependent variable led to decisions about what variables were included in the regression equation (Mertler \& Vannatta, 2013). DAS was the only variable that was significantly correlated with QOL and therefore it was included in the regression equation. Age and ethnicity 
were included in the regression equation since they were the demographic variables. Masculinity, being significantly correlated with DAS, will be included to see how it contributes to QOL. There was a significant negative correlation between urinary and sexual functioning; however, these two variables were not correlated with any of the other independent or dependent variables. For this reason, one of them was eliminated. Founded on the literature that has documented sexual functioning as a major problem for men with prostate cancer, urinary functioning was not included in the regression equation (Hoznek et al., 2005; Wei et al., 2002).

Hierarchical multiple regression was conducted using the Enter method. Age, ethnicity, masculinity, sexual functioning, and relationship with partner were entered into the regression equation. Tolerance statistics and measure of multicollinearity indicated that all independent variables were greater than .1 and therefore tolerated in the model. The overall regression model was significant $(F(5,91)=2.39, p=.04)$ and accounted for $11.8 \% \%$ of variance in QOL $\left(\mathrm{R}_{\text {adj }}^{2}=.07, \Delta \mathrm{R}^{2}=.12, p=.04\right)$. Examination of the beta weights suggested that DAS ( $\beta=-.25, t(91)=-2.28, p=.03$ ) significantly contributed to the model (see Table 8). 
Table 8

Relative Contributions to $Q O L$ in Men with Prostate Cancer

\begin{tabular}{|c|c|c|c|c|c|}
\hline Variable & $B$ & SEB & $B$ & $T$ & $p$ \\
\hline Age & .15 & .12 & .13 & 1.32 & .19 \\
\hline Ethnicity & 2.76 & 1.94 & .14 & 1.42 & .16 \\
\hline $\begin{array}{l}\text { Relationship with } \\
\text { partner }\end{array}$ & -.22 & .10 & -.25 & -2.28 & $.03^{*}$ \\
\hline Masculinity & -.20 & .15 & -.15 & -1.38 & .17 \\
\hline Sexual functioning & .12 & .11 & .11 & 1.14 & .26 \\
\hline
\end{tabular}

Note. Ethnicity was dummy coded as nonHispanic "0" and Hispanic "1"; relationship with partner was measured using DAS, masculinity was measured using CMNI, sexual functioning were measured using UCLA PCI. 


\section{CHAPTER V \\ DISCUSSION}

Quality of life is an essential outcome measure for the quality and satisfaction of care provided to patients (Montazeri, 2009; Wilson \& Cleary, 1995). Patients diagnosed with chronic conditions discuss their desire to have their health conditions treated and aspire to retain their physical functioning and social roles. Enhanced QOL also contributes to improved mortality rates (Chida \& Steptoe, 2008). This study focused on examining the relationship between age, ethnicity, sexual dysfunction, urinary incontinence, masculinity, and perception of the relationship with the partner on the QOL in men diagnosed with prostate cancer. Prostate cancer, the leading cause of cancer in men, has positive survival rates and constitutes a challenge with its side effects (Jemal et al., 2006; Jemal et al., 2010; Siegel et al., 2013). These men are generally around 50 years of age and older, do not look or feel sick, but endure urinary and sexual dysfunctions as a result of their prostate cancer (Hoznek et al., 2005; Wei et al., 2002). Consequently, the normalcy of their life is disrupted, and their QOL is disputed (Howlet et al., 2010; Monga, Kerrigan, Thornby, Monga, \& Zimmermann, 2005). Research with men with prostate cancer has suggested that the disease and treatment side effects contribute to issues with physical functioning, relationships, social roles, and QOL (Kaasa, Mastekaasa, \& Lund, 2003). Studies have discussed the relationship between prostate cancer treatment side effects on each of masculinity (Burns \& Mahalik, 2006; Fergus, Grey, \& Fich; 2002), partner relationship (Galbraith, Pedro, Jaffe, \& Allen, 2008; Sanders et al., 2006), and QOL (Howlet et al., 2010; Monga, Kerrigan, Thornby, Monga, \& Zimmermann, 2005) alone. However, few studies have specifically focused on the 
relationships among prostate cancer treatment side effects (i.e., sexual dysfunction, urinary incontinence), masculinity, and relationship with the partner together on QOL in men.

South Florida, being a multiethnic region, constituted an interesting setting for this study. The majority of research examining the QOL in men with prostate cancer has been conducted with predominantly Caucasian samples of men. However, this study included men from minority ethnic populations such as Latin American and Caribbean Hispanic origins in addition to other ethnicities (i.e., Cubans, Venezuelans, Columbians, Haitians, and others). Another demographic specificity of South Florida includes the increased numbers of retirees and older adults which was reflected in the average age of men in this study. As such, this study was fundamental in this setting since older men are at a higher risk for developing prostate cancer (PCF, 2011), and they have a different experience with regards to their perceptions of QOL (Harden, Northouse, \& Mood, 2006;

Oliffe, 2005).

\section{Discussion of Findings}

\section{Age}

The average age of men in this sample was close to 70 years and ranged from 52 to 85 years. With prostate cancer risk increasing after the age of 50 years, the older age of the sample was expected (ASC, 2011; PCF, 2011). Additionally, $16 \%$ of the population in Miami is reported to be above the age of 65 years old (U.S. Census, 2010). With the increased percentage of older people in Miami, the health care concerns and QOL perspectives of this population is essential. This sample contributed to the understanding of the QOL concerns of older men with prostate cancer in Miami. 
The sample in this study was older than many of the samples in the majority of studies on prostate cancer and treatment side effects (Choo et al., 2010; Penson et al., 2005; Resnick et al., 2013; Sacco et al., 2006; Stanford et al., 2000). Few studies have included samples of men who had a similar age range or older (Harden et al., 2006; Hawes et al., 2006; Stansburry et al., 2003). With age being a significant risk factor for prostate cancer (PCF, 2011), it is important to understand differences in priorities of men regarding their QOL in men who are 65 years and older especially that they constitute $65 \%$ of men diagnosed with the disease (PCF, 2011).

Studies that have examined men with prostate cancer and masculinity (Fergus et al., 2002; Grunfed et al., 2013; Oliffe, 2005) and partner relationships (Boehmer \&Clark, 2001; Fergus et al., 2002; Galbraith et al., 2008; Gray et al., 2000; Sanders et al., 2006) also included samples of men who were younger than the men in this sample. Harden and colleagues (2006) suggested that age contributed to men's perspectives of relationships, intimacy, and other issues. However, this study showed that age did not contribute to men's perceptions of relationship dynamics. It is vital to understand the perceived QOL in men with prostate cancer in an aging population as it was estimated that the number of Americans aged 65 and older are expected to double in the next 25 years (CDC, 2013). Additionally, the increased racial and ethnic diversity in older adults was projected to increase, and the proportion of Hispanic older adults is anticipated to be $12 \%$ and $20 \%$ in 2030 and 2050 compared to the reported proportion of $7 \%$ in 2010 (CDC, 2013). Thus, this study contributed to understanding the QOL perspectives of older multiethnic men with prostate cancer. 
Age was has been shown contribute to increased reporting of urinary incontinence (Sacco et al., 2006) and sexual functioning (Rondorf-Klym \& Colling, 2003; Penson et al., 2005; Sanda et al., 2008). However, findings from this study did not indicate that older age affected the presence of urinary and sexual dysfunctions. Questions in this study did not directly ask about the beliefs and engagement of older men regarding sexual functioning. Nevertheless, many men reported the use of sexual enhancement regimens which could be suggestive of an interest in sexual activity. These results can challenge the findings of Oliffe (2005) who reported that older men were expected to have less emphasis on sexuality. Then again, during the administration of the surveys, men would not emphasize in their discussions with the researcher their sexual dysfunction and focus on other QOL issues such as their relationships with their partners (Harden et al., 2006). This reluctance to disclose sexual dysfunctions was similar to the inicidental findings reported by Oliffe (2005).

\section{Ethnicity}

Men in this sample were generally Hispanic who mostly were born in the United States, Cuba, Haiti, Columbia, and Venezuela. The majority of men reported that their families were originally from Cuba and Haiti. These findings were reflective of the $65.75 \%$ Hispanic population in Miami of which $34.14 \%$ came from Cuba as reported by the U.S. Census Bureau 2010. Interestingly, more than half the population in Miami was reported to be foreign born (U.S Census). This was a significant finding since few previous studies have examined the QOL in Hispanic men with prostate cancer (Stanford et al., 2010). Understanding the perceptions of Hispanic men diagnosed with prostate cancer is important for healthcare providers in multiethnic and multiracial Miami to 
provide better care. These findings were unique since the majority of Hispanics were Cuban or of Latin American decent, unlike the Hispanics or Latinos in studies reported in the literature who were predominantly of Mexican descent. Distinctively, this sample included a number of men from Haiti and those who identified themselves as Black Caribbean which is a growing population in Miami and the United States; however, this demographic trend has not been represented in the literature. Future research would benefit from examining the contributions of QOL in men from different racial and ethnic groups that are growing in the United States.

Despite African American men having the highest risk for prostate cancer, few African American men participated in this study. However, the representation of African American men in this study (14.7\%) was representative of the demographics of Miami of $22.31 \%$ (U.S. Census, 2000). Few studies have compared the outcomes of men with prostate cancer from different racial and ethnic backgrounds (Stanford et al., 2000). Stanford and colleagues (2000) discussed better sexual functioning in African American and Hispanic men compared to Caucasian men after undergoing surgery. The findings from this study did not show any difference in outcomes (i.e., urinary and sexual functioning) between Hispanic and non Hispanic men. Additionally, there were no differences in choice of treatment or use of sexual enhancement regimens.

In a sample that was predominantly Hispanic, an emphasis on machismo and caballerismo was anticipated. As a result, Hispanic men were projected to emphasize the socially constructed Hispanic male gender role thus reflecting hypermasculinity such as increased strength, family protection, and sexual prowness. Quite the opposite, this study showed that Hispanic and nonHispanic men had similar reports about sexual problems, 
use of sexual enhancement regimens, and sexual dysfunction. It is worth noting that the majority of men did not answer the question about their sexual functioning prior to the diagnosis of prostate cancer. This unanswered question might have been a reflection of machismo since a little less than half of the participants who answered reported some problems. Subsequently, the questions remain: Did machismo decreases with age?, or Did the disease process make these men redefine quantity versus quality of life? (Harden et al., 2006). These questions could be at the foundation of future research. Furthermore, men in this study were married or partnered; hence they were believed to have a source of support that may explain the argument of exhibiting less emphasis on sexual prowness.

\section{Sexual Dysfunction}

Sexual dysfunction after the diagnosis and treatment of prostate cancer was reported to be an inevitable consequence (Hoznek et al., 2005; Penson et al., 2005;

Stanford et al., 2000; Wilt et al., 2008). The majority of men in this study reported sexual dysfunction similar to rates that have been reported in the literature (Alemazoffar et al., 2011; Hoznek et al., 2005; Penson et al., 2005; Sacco et al., 2006; Sanda et al., 2008; Wilt et al., 2008). Decreased potency and the inability to perform sexually were considered to be of the most bothersome consequences of the disease (Meyer et al., 2003; Monga et al., 2005; Moul et al., 2001). Irrespective of the treatment option, studies that have followed men more than 18 months after prostate cancer have reported sexual dysfunction (Alemazoffar et al., 2011; Choo et al., 2010; DiBlasio et al., 2008; Stanford et al., 2000).

The findings from this study emphasized that men endure sexual issues and problems after the diagnosis and treatment of prostate cancer. Most men reported 
decreased sexual desire, erection problems, and the inability to reach orgasm. Erectile dysfunction was established to be the main reason behind these sexual issues and dysfunctions. Few men in this study discussed that they can have and/or maintain an erection that is adequate for sexual intercourse. Data about sexual functioning prior to prostate cancer was not insightful of the men's status since the majority did not answer the respective question. Research has suggested that there was a relationship between sexual functioning before and after prostate cancer (Alemazoffar et al., 2011; Moul et al., 2001; Penson et al., 2005). Men who had better sexual functioning at baseline were found to have better sexual functioning after prostate cancer treatment when compared to men who reported baseline sexual dysfunction (Alemazoffar et al., 2011; Moul et al., 2001; Penson et al., 2005). However, this study could not confirm the findings from the literature. Participants who reported having sexual problems prior to the diagnosis of prostate cancer had similar sexual issues as those who did not have sexual problems prior to prostate cancer. Baseline sexual functioning had no association with the dysfunction issues endured after prostate cancer. Future studies examining the impact of baseline sexual functioning on their post treatment status in Hispanic men is essential. Additionally, using a mixed methods study to answer this research question would provide a full view of the issue.

Despite the reported sexual issues, around half of the sample reported having had intercourse during the last month. As such, men were still trying to maintain a sexual experience which could be explained by the discussions about the importance of sexual activities by men (Oliffe, 2005). This was an interesting finding since the majority of men shared that they considered that their sexual functioning was a major problem for 
them, and very few were able to have an erection whenever they wanted. Men in this sample were married or partnered and, therefore, sexual performance may have been considered to be an expectation. Hence, engagement in sexual activities was important for these men. Many men were using sexual enhancement regimens to support their poor performance. Penson and colleagues (2005) suggested that most men diagnosed with prostate cancer use sexual enhancement regimens, namely Viagra ${ }^{\circledR}$, vacuum erection devices, and intracavernous injections. The use of these sexual enhancement regimens was found to assist in men's satisfactory sexual performance (Incrocci et al., 2001; Penson et al., 2005). However, the use of sexual enhancement regimens by men in this study did not enhance the sexual performance of men.

Age was also suggested to influence the sexual performance of men after prostate cancer (Alemazoffar et al., 2011; Choo et al, 2010; Penson et al., 2005). Men 60 years old or younger were found to have a lower risk of developing sexual dysfunction (Moul et al., 2010). Men older than 60 years had issues returning to baseline sexual functioning (Alemazoffar et al., 2011; Choo et al, 2010; Penson et al., 2005). Findings from this study did not show that age influenced sexual function or dysfunction of men. Nonetheless, these men were generally older than men included in other studies and therefore this sample might have highlighted a population that is older and therefore has similar sexual issues. The lack of a wider age range in the sample might have contributed to undermining the contributions of age to the sexual dysfunction of men.

Few studies reported in racial and ethnic differences in sexual outcomes after prostate cancer treatment (Alemazoffar et al., 2011; Penson et al., 2005; Siegel et al., 2001). Race/ethnicity was found to contribute to the sexual functioning after prostate 
cancer surgery. White men were reported to have decreased potency more than African American and Hispanic men (Siegel et al., 2001). Findings from this study showed that there were no differences between Hispanic and nonHispanic men after treatment for prostate cancer. Despite the norms of sexual prowness and machismo exhibited by Hispanic men, findings did not corroborate the anticipated emphasis on sexual functioning.

\section{Urinary Dysfunction}

Dribbling or urinary incontinence are common side effects after the treatment of prostate cancer (Penson et al., 2005; Stanford et al., 2000). Studies defined the rate of incontinence by identifying the need to use continence pads in addition to their frequency (Sacco et al., 2006). Over time, studies have reported an improvement of urinary continence (Lepor et al., 2004; Penson et al., 2005; Stanford et al., 2000; Wei at al., 2002). Findings from this study showed that around half of the sample complained of urinary dribbling every day. The other half of the sample was divided between occasional dribbling and total loss of bladder control. So, the majority were using continence pads at least once daily. The literature has suggested that at 18 months and on, men reported major improvements in their urinary functioning (Lepor et al., 2004; Sacco et al., 20006). This improvement was not identified in this cross sectional study. The majority of men in this study were diagnosed with prostate cancer more than 1 year ago. However, men were not asked whether they had urinary issues such as dribbling or decreased control prior to their diagnosis. Additionally, this study did not assess the improvement of urinary functioning from the time of initiation of the prostate cancer treatment. Future studies should consider assessing baseline urinary functioning in 
addition to status immediately after initiation of treatment and forward. Studies have suggested rates of more than $80 \%$ improvement in urinary dysfunction with time (Sacco et al., 2006; Stanford et al., 2000), but urinary dysfunction can continue to be present up to 5 years after the treatment (Palmer et al., 2003). However, this study showed that men diagnosed with prostate cancer more than 2 years prior had more urinary issues than those diagnosed 6 months to 1 year ago. This finding was not in accordance with what has been reported in the literature. It is worth noting that men were asked about their urinary functioning in relation to the time since diagnosis of prostate cancer and not since the initiation of treatment of prostate cancer. Identifying the time of initiation of treatment may contribute to explaining these results. Overall, the urinary dysfunction in this study was perceived to be a moderate to big problem.

This study did not investigate the effect of dribbling on different activities of daily life. However, urinary dysfunction was suggested to interfere with men's sexual performance and as such was considered to be a moderate to big issue. Research has discussed the prevalence or dribbling and stress incontinence in men with prostate cancer that may occur during sexual intercourse (Palmer et al., 2003). Consequently, urinary incontinence may be a source of embarrassment and disruptive to sexual performance. Hence, men in this study identified that urinary and sexual dysfunctions were associated with each other. Perhaps this association could be the result of their concurrence as a side effect of the treatment options (Potosky et al., 2000; Wei et al., 2002) or due to the interference of urinary dysfunction with sexual performance (Palmer et al., 2003).

Age has been found to influence the return of urinary functioning to baseline (Penson et al., 2005; Sacco et al., 2006; Stanford et al., 2000). Studies have shown that 
older men had slower or no improvement of their urinary functioning after the treatment of prostate cancer. The decreased prognosis of regaining continence was explained by age and possible presence of comorbidities that may add to the cause of incontinence (Penson et al., 2005; Sacco et al., 2006; Stanford et al., 2000). Findings from this study showed that age did not have any association with urinary functioning of men with prostate cancer. Again, similar to the discussion related to sexual functioning, a primarily older sample of men might have decreased the presence of variability in age and urinary functioning.

Research has not emphasized the difference in urinary functioning among men from different racial/ethnic groups. This study suggested that there were no differences in urinary functioning between Hispanic and nonHispanic men. Findings showed that there were differences in urinary functioning in men with advanced stages of prostate cancer. This can be as a result of the treatment option or the initiation of multiple treatment options. Research has not emphasized urinary functioning of men with respect to the stage of prostate cancer. Also, men who underwent hormonal therapy reported more urinary issues than men who underwent prostate surgery. This was contrary to what was has been reported in the literature (Basario et al., 2002; DiBlasio et al., 2008; Monga et al., 2005; Potosky et al., 2000, Stanford et al., 2000). Perhaps men in this study started hormonal therapy as a second line treatment option or as a result of recurrence of the disease. Men might have selected hormonal therapy as their current treatment option and did not consider themselves as having multiple treatments since they have undergone one option at a time. Future studies should consider clarifying the question about treatment options to include prior and current multiple treatment options. 


\section{Masculinity}

Masculinity is a socially constructed expectation that embrace a set of norms and behaviors that are anticipated to be exhibited by men (Connel, 2010; Kimmel, 2000). These norms and beliefs can be influenced by environmental factors as well as social and cultural beliefs (Connel, 1995). Men not complying by these expectations have been viewed to be subordinated and weak (Charmaz, 1995; Connel, 1995, Ferrel, 1993; Oliffe, 2005). Masculine norms also reflect on health care perceptions and practices (Addis \& Mahalik, 2003; Mahalik et al., 2007). Of the many expectations of a masculine man, sexual prowness and ability to satisfy a partner are crucial qualities (Gray et al., 2002; Khan et al., 2008; Kimmel, 2000). Most studies that have examined the meanings of masculinity in men with prostate cancer were qualitative in nature. These studies focused on relating masculine norms to the perception of sexual and urinary functioning after the diagnosis and treatment of prostate cancer (Burns \& Mahalik, 2008; Chapple \& Ziebland, 2002; Fergus et al., 2002; Oliffe, 2005).

Men with prostate cancer discussed their discomfort in revealing to their physician symptoms that could undermine their strong masculine image (i.e., sexual and urinary dysfunctions) (Mahalik et al., 2007; O’Brien et al., 2005; Oliffe \& Thorne, 2007). Sexual dysfunction was considered to be a challenge that led to feelings of shame and embarrassment (Fergus et al., 2002). In men with strong masculine norms, sexual dysfunction was found to negatively influence men's social and role functioning (Burns \& Mahalik, 2008). As a result, men perceived the diagnosis of prostate cancer and the related sexual dysfunction as a threat to their masculinity and image (Fergus et al., 2002). The use of sexual enhancement regimens was recognized to support men's performance; 
however, the consequential sexual experience was reported to lose its spontaneity and was described as a disruptive experience (Fergus et al., 2002). Urinary functioning was also identified to be a symptom that would cause embarrassment since it necessitated frequent bathroom breaks. Additionally, the fear of urinary leak threatened men's masculine image and how comfortable they were with their social role (Gronfed et al., 2013).

Findings from this study showed that conformity to masculine norms was not influenced by sexual functioning, urinary functioning, or QOL. The anticipated values of machismo and strong masculine norms were thought to be prevalent in Hispanic men, thus influencing the impact of sexual and urinary dysfunction on the QOL in men with prostate cancer. However, the findings did not support this premise. Studies suggested that older men placed less emphasis on the impact of sexual dysfunction on their lives (Chapple \& Ziebland, 2002; Oliffe, 2005). Therefore, a generally older sample of men might not place emphasis on decreased sexual functioning. On the other hand, it might be the machismo trait that promoted men to report that their sexual issues were not affecting their QOL. The fact that men in this study were married or partnered also provided them with a supportive medium. Research has demonstrated that wives were the main source of social support for men diagnosed and treated for prostate cancer (Giarelli et al., 2003; Gray et al., 2002).

\section{Relationship with the Partner}

Female partners have been recognized to be an essential source of support to men with prostate cancer (Gray et al., 2002). The diagnosis of prostate cancer did not only affect men, it also influenced the QOL of their female counterparts (Badr \& Carmack 
Taylor, 2009; Galbraith et al., 2008; Giarelli et al., 2003; Gray et al., 2002; Hawkins et al., 2009). In other words, it was considered to be a diagnosis for the couple. Sexual dysfunction and issues have caused relationship problems (Hawkins et al. 2009) and a transformation in the woman's role within the relationship (Badr \& Carmack Taylor, 2009). Women found themselves assuming the role of the care giver and losing their feminine sexual image of the lover (Badr \& Carmack Taylor, 2009; Hawkins et al., 2009). Sexual performance was perceived to be essential for a harmonious relationship. With decreased sexual abilities, intimacy issues arose that contributed to decreased satisfaction with the relationship (McCabe \& Matic, 2008). As a result, couples discussed the development of problems and issues within the relationship that were the result of decreased communication and intimacy (Badr \& Carmack Taylor, 2009; Galbraith et al., 2008; Giarelli et al., 2003; Gray et al., 2002; Hawkins et al., 2009).

Findings from this study showed that the relationship satisfaction was important for men diagnosed and treated for prostate cancer. However, relationship satisfaction was not affected by the sexual and urinary dysfunction caused by prostate cancer treatment. Despite the high prevalence of sexual dysfunction, men's perceived relationship with their female partners was not affected. These findings were contrary to what has been reported in the literature (Badr \& Carmack Taylor, 2009; Galbraith et al., 2008; Giarelli et al., 2003; Gray et al., 2002; Hawkins et al., 2009). Men’s decreased sexual functioning was confounded by a response that contributed to decreased communication and intimacy (Boehmer \& Clark, 2001; Hawkins et al., 2009; McCabe \& Matik, 2008) that led to decreased relationship satisfaction (Hawkins et al., 2009; McCabe \& Matik, 2008). Studies discussed a change in roles in the relationship that 
might have predisposed the decreased satisfaction with the relationship (Fergus et al., 2002; Galbraith et al., 2008; Giarelli et al., 2003; Gray et al., 2002; Hawkins et al., 2009). Women were perceived to be a major source of social support (Giarelli et al., 2003), but at the same time they lost their sexual image (Badr \& Carmack Taylor, 2009; Harden et al., 2002). Women reported that they were no longer feeling that they were the lovers and, therefore, intimacy was scarce (Sanders et al., 2008).

Men in this sample were older, and the majority had been in their current relationship for more than 1 year. As a result, these couples have lived with the prostate cancer diagnosis and treatment side effects; hence, they may have developed coping strategies. On another note, older men were found to discuss the interruption of life activities that is caused by sexual and urinary functioning (Harden et al., 2006). However, they still reported decreased relationship satisfaction (Harden et al., 2006) and emotional burden on the couple (Fergus et al., 2002; Galbraith et al., 2008). Thus, the diagnosis and treatment of prostate cancer caused a challenge to the relationship and subsequently affected the QOL of these men.

\section{Quality of Life}

Quality of life is a measure of disease and treatment outcome in addition to being a basic human right in health care (U.S. Department of Health \& Humans Services, 1990). Being a multidimensional concept (Cella 1994), QOL is considered to refer to the normal functioning and general wellbeing including physical, social, psychological, and other dimensions that would lead to an overall satisfaction with life (Ferran et al., 2005). Men with prostate cancer have positive survival rates (Jemal et al., 2006; Jemal et al., 2010; Siegel et al., 2013) but endure sexual and urinary dysfunctions (Penson et al., 2005; 
Stanford et al., 2000) that have an impact on their QOL (Howlet et al., 2010; Penson et al., 2003; Potosky et al., 2004; Rondorf-Klym \& Colling, 2003; Sanda et al., 2008). Sexual and urinary dysfunctions were found to affect the practicality of resuming life routines due to the need of frequent bathroom breaks (Gronfed et al., 2013) in addition to affecting their masculine image (Burns \& Mahalik, 2008; Fergus et al., 2002; Gray et al., 2002; Oliffe, 2005) and the status of the relationship with the partner (Galbraith et al., 2008; Hawkins et al., 2009; McCabe \& Matic, 2008; Sanders et al, 2008) as a result of their inability to perform sexually.

Irrespective of the treatment option, men endured sexual and urinary dysfunctions and as a result their QOL was challenged (Bradeis, et al., 2000; Sanda et al., 2008). Findings from this study suggested that there were no differences in QOL among men undergoing surgery, radiation, hormonal therapy, watchful waiting, or multiple treatments. Moreover, the majority of men complained of sexual and urinary dysfunctions in a similar manner and therefore the treatment option did not offer any functional outcome differences (Bradeis, et al., 2000; Monga et al., 2005; Penson et al., 2003; Sanda et al., 2008; Stanford et al., 2000 Wei et al., 2002). These results supported the notion that the treatment of prostate cancer is an individualized plan based on the patient's wishes and disease status. Interestingly, only half the men in this study participated in choosing the treatment option or had their preferences discussed. Additionally, men's concerns were not discussed in relation to what to expect after the treatment of prostate cancer. These results highlighted the communication issues between men and their physicians discussed in the literature where both men were not 
elaborate in discussing the treatment expectations prior to the choice of the treatment option (Oliffe \& Thorne, 2007).

Men in this study considered their health to be good or very good. Few men considered their health to have deteriorated during the past year. Studies with men with prostate cancer treatment side effects have suggested that sexual and urinary dysfunction were not considered to decrease the general health ratings of older men (Bradeis, et al., 2000; Monga et al., 2005; Penson et al., 2003; Sanda et al., 2008; Stanford et al., 2000 Wei et al., 2002). Men believed that sexual dysfunction was bothersome thus making them feel that their general health was jeopardized (Penson et al., 2003; Sanda et al., 2008; Stanford et al., 2000 Wei et al., 2002).

Studies have examined the perceived QOL of men based on their baseline sexual functioning prior to the diagnosis of prostate cancer (Alemozaffar et al., 2011; Chen et al., 2009). Results from this study showed that men with sexual problems prior to the diagnosis of prostate cancer had lower QOL. These findings were contrary to what has been reported by other studies (Alemozaffar et al., 2011; Chen et al., 2009) especially research that suggested that baseline sexual and urinary functioning predicted functioning after the treatment (Alemozaffar et al., 2011; DiBlasio et al., 2008; Penson et al, 2005). This study did not show that baseline functioning affected post treatment sexual functioning. Men with no sexual issues prior to the diagnosis of prostate cancer might have been comforted by being treated for the disease and having positive survival rates. As a result, their QOL, after the treatment and despite enduring sexual and urinary side effects, was reflective of their contentment of having a positive prognosis. On the other hand, those who had sexual issues at baseline might have been struggling with their QOL 
and with the added challenge of prostate cancer, their QOL was additionally affected. Future research is warranted to further understand the changes of sexual and urinary functioning from baseline and the effects on men's QOL.

The use of sexual enhancement regimens was common among men in this study. Men who used medications, pills, and intracavernous injections were found to have a better QOL than those who had penile implants. Studies have not emphasized QOL outcomes comparing sexual enhancement regimens. However, medications, pills, and intracavernous injections are methods that might provide a sexual experience that is closer to the baseline norm to which the men were accustomed. Studies have discussed the discomfort that men had when their sexual performance did not feel normal or was not a spontaneous performance (Bakhour et al., 2001; Fergus et al., 2002; Galbraith et al., 2008; Oliffe, 2005). Future research examining the association between the different sexual enhancement regimens on men's QOL is important to better direct men on what option would be best for them.

With respect to the effect of urinary functioning on the QOL of men, results showed that men who have more incontinence issues reported decreased social functioning. These results were similar to findings reported by previous studies (Harden et al., 2006; Gronfed at al., 2013). Having to take frequent bathroom breaks affected men's social life at home (Harden et al., 2006) and at work (Gronfed at al., 2013). Men discussed having to change lifestyles or interrupt activities needing bathroom breaks or fearing the embarrassment of urinary leakage. As a result, their normal life activities were broken up that was reflected on their decreased social functioning. 
Sexual dysfunction was considered to be the most important outcome of prostate cancer (Howlet et al., 2010; Penson et al., 2003; Potosky et al., 2004; Sanda et al., 2008). Accordingly, sexual issues were found to be directly related to men's ratings of their general health. Considering that sexual functioning is important for men (Fergus et al., 2002; Meyer et at., 2003), inability to perform sexually can be perceived as a health condition. Hence, men considered their general health to be affected. This finding highlights the importance of sexual functioning in relation to men's perception of their overall health.

Examinination of QOL includes assessing the physical and mental health functioning of men. Findings showed that there was an association between physical and mental health functioning in men with prostate cancer. Men with decreased physical health functioning reported decreased mental health functioning. As a result, physical functioning was related to these men's vitality and ability to feel well enough to continue their routine daily life activities. Having role limitations as a result of physical functioning, men's mental health and emotional status were affected and so was their social functioning. Research has suggested that sexual and urinary dysfunctions were found to impact the physical functioning of men and therefore negatively influence their QOL (Howlet et al., 2010; Steineck et al., 2002). As a result, these men believed that they cannot fulfill their expected role of being a sexual being and abiding by their perceptions of masculinity (Bokhour et al., 2001; Chen et al., 2009; Howlet et al., 2010). Furthermore, they believed that normal life activities had to be interrupted and planned around their urinary dysfunction and fear of urinary leakage (Fergus et al., 2002; Fronfed et al., 2010; Korfage et al., 2008). Accordingly, their QOL of life was challenged which 
was reflected on their relationship satisfaction. With men perceiving that they cannot perform their expected role in the relationship, they considered that the relationship was not satisfactory anymore (Badr \& Carmack Taylor, 2009; Galbraith et al., 2008; Giarelli et al., 2003; Gray et al., 2002; Hawkins et al., 2009).

\section{Perceived Impact on QOL}

Quality of life, being a multidimensional concept, is influenced by a multitude of factors including individual characteristics, symptoms, general health perceptions, environmental and social factors, and others (Ferrans et al., 2005). In view of this conceptualization, QOL in men with prostate cancer was hypothesized to be influenced by age, ethnicity, prostate cancer treatment side effects (i.e., sexual and urinary dysfunction), masculinity, and perceived relationship with the partner. Studies emphasized the challenges that men face when dealing with sexual and urinary dysfunction (Howlet et al., 2003; Penson et al., 2003; Potosky et al., 2004; Rondorf-Klym \& Colling, 2003). Additionally, the majority of these studies discussed the influence of these side effects on the QOL of men irrespective of the treatment option (Howlet et al., 2003; penson et al., 2003; Potosky et al., 2004; Rondorf-Klym \& Colling, 2003). These side effects also had an effect on the masculine image of men (Burns \& Mahalik, 2008; Fergus et al., 2002; Gray et al., 2002; Oliffe, 2005) and their relationship satisfaction (Galbraith et al., 2008; Giarelli et al., 2003; Gray et al., 2002; Hawkins et al., 2009). Relationship satisfaction was also found to be related to men's perceived QOL (Bokhour et al., 2004; Sanda et al., 2008).

Findings from this study showed that there was a relationship between sexual and urinary functioning. Men who had more sexual issues reported less urinary dysfunction. 
The literature has discussed the extent of sexual and urinary dysfunction in relation to treatment option and across time (Penson et al., 2003; Potosky et al., 2004; Sanda et al., 2008). These findings showed no functional differences among different treatment options. However, it can be explained that men with sexual problems might have considered them as a greater problem (Penson et al., 2003; Steinek et al., 2002).

Prostate cancer treatment side effects were reported to challenge men's masculinity (Burns \& Mahalik, 2008; Fergus et al., 2002; Gray et al., 2002; Oliffe, 2005) and relationship satisfaction (Galbraith et al., 2008; Giarelli et al., 2003; Gray et al., 2002; Hawkins et al., 2009). Findings from this study showed that men with stronger masculine norms were less satisfied with their relationship. Stronger masculine norms were reflected by beliefs of sexual prowness and ability to satisfy a partner sexually (Gray et al., 2002; Khan et al., 2008; Kimmel, 2000). The inability to comply with these perceived social norms might allow them to feel less of a man (Chapple \& Ziebland, 2002; Connel, 2010; Fergus et al., 2002; Kimmel, 2000; Oliffe, 2005). Accordingly, they believe that their role within the relationship was be jeopardized. Stronger masculine norms were usually aligned with the concepts of hypermasculinity and machismo. In a sample that was predominantly of a Hispanic background, this finding suggests that machismo may still govern how men view their relationship. Men with strong beliefs in complying with the values and norms of machismo would expect to maintain their strong role in the relationship. Having sexual and urinary dysfunction would cause a threat to their role and therefore they might perceive that they are less satisfied with their relationship. That is, these men must be struggling to maintain their image as a strong man in the relationship and therefore problems might arise. Issues of communication and 
intimacy were discussed in the literature among men with prostate cancer (Badr \& Carmack Taylor, 2009; McCabe \& Matic, 2008). As such, the inability of men to live up to their expected role might heighten intimacy and communication issues and therefore cause a challenge to the relationship. Future research is needed to help understand the dynamics and perceptions of men's roles within their relationship after the diagnosis and treatment of prostate cancer.

Results suggested that men with better relationship satisfaction reported decreased QOL. This finding was opposite to what was discussed in the literature where better partner relationships was related to improved QOL as a result of the supportive role that women provided (Galbraith et al., 2008; Giarelli et al., 2003; Gray et al., 2002; Hawkins et al., 2009). Again, the concepts of machismo might have created a pressure on men to abide by their expected role. Therefore, men with an unsatisfactory relationship might not have had to challenge themselves to maintain their image and therefore accepted their treatment side effects and reported a better QOL. On the other hand, men who had a good relationship with their partner might have been eager to maintain their machismo image and were bothered by their treatment side effects. These side effects were thought to pose a threat to the harmony of their relationship. As a result, they were not able to accept their sexual and urinary dysfunctions and hence reported decreased QOL.

In the end, what mattered most to the QOL of men with prostate cancer was their relationship with their partner. Their female counterpart was a major source of support who influenced their perception of QOL. Similar to what has been reported in the literature, the partner relationship is important for men diagnosed with prostate cancer (Galbraith et al., 2008; Giarelli et al., 2003; Gray et al., 2002; Hawkins et al., 2009). The 
majority of men in this study were in their current relationship and had prostate cancer for more than one year. Therefore, they may have developed an understanding of how their treatment side effects were going to affect their life. The literature has emphasized the supportive role of the female partners of men with prostate cancer (Galbraith et al., 2008; Giarelli et al., 2003; Gray et al., 2002; Hawkins et al., 2009) which in turn directly influenced their QOL. An in-depth appreciation and proper communication between the couple would allow men to be adjusted to their treatment side effects. Accepting their treatment side effects and realizing the changes and challenges that they would have to live with was stressful on their relationship. Also, finding ways to deal with their side effects and maintaining a strong relationship would lead to an improved QOL.

\section{Limitations of the Study}

Several potential limitations of this study were identified. This was a crosssectional design and self-administered survey study and therefore the findings represent the perception of men at one point in time. Thus, generalizations and conclusions about changes or trends over time cannot be made. Additionally, the survey included recall questions about sexual problems prior to the diagnosis and functional outcomes up to 4 weeks prior to answering the survey. There might have been a recall bias in some of the

responses. A convenience sample was used to collect data and consequently there might have been a self-selection bias. Data about men who did not choose to volunteer or accept to participate in the study were not collected. This potential bias in sampling would also limit the ability to generalize findings.

The self-administered surveys might have resulted in response bias or social desirability bias. In other words, the questions included sensitive information about 
sexual and urinary functioning, partner relationship issues, and masculine norms. Therefore, men might have provided some answers that would have been socially acceptable and desirable; thus posing potential inaccuracies of the results. The media presents the image of a strong older man at older ages and presents sexual enhancement regimens and gadgets. As a result, men are cultured into what is their socially expected masculine image. Additionally, Hispanic men are also confronted with the norms of machismo which add to what a "real" man should believe. These concepts might have biased men's answered to some of the survey questions.

Also, self-administered survey included self-identified men who do not have sex with men. Emphasis was not made to exclude bisexual and transgender men. Inclusion criteria focused on men who reported to be in a relationship with a woman and who do not identify themselves as men who have sex with men. There might be social desirability and inaccurate reporting bias that might have influenced the results. Future research would benefit from examining the QOL in gay, bisexual, and transgender men diagnosed with prostate cancer.

Data was collected using a diverse sample of men who are from different ethnic and racial backgrounds. There might be intraethnic differences within the Hispanic cuture that might have influenced the results. However, the sample size was small to be able to identify groups and compare QOL in men from different ethnic backgrounds. Therefore, generalizations about the different ethnicities can be suggested but cannot be generalized. Few men were born in the United States, whereas many men migrated during their early or late adulthood. Data about the date and of migration to the U.S. was not collected. Also, information about acculturation was not gathered. Therefore, there 
might be acculturation and cultural differences within the same ethnicity especially between men who were born in the U.S. and those who were not. Future research is warranted to examine the contributions of culture and acculturation to QOL in men with prostate cancer.

Another major issue that was encountered during the course of the study was the ability to obtain the support ("buy in") of physicians to allow recruitment of clients at their clinics. Many urologists did not feel comfortable with their clients participating in a research study that was not administered by their clinic. Hence, the clients were recruited from clinics who accepted that a previously agreed on number of clients to participate in the study. Therefore, the convenience sample that was included could have been biased by the timing and clinics where the study was conducted. This adds to the issues that challenge the ability to generalize the findings of this study.

Studies have examined masculinity using qualitative research designs. This study used the CMNI which has been psychometrically tested (Mahalik et al., 2003). However, not many studies have used the short version which was used in this study. Additionally, the tool was translated to Spanish and used for the first time in this study. As a result, comparison with other studies and the ability to generalize the findings is not possible.

The study included psychometrically tested instruments that assessed the concepts at one point in time. As such, the development of a timeline that explained the trend or course of the events and perceptions along the course of diagnosis and treatment of prostate cancer could not be achieved. Also, only men were interviewed. The exclusion of the female counterparts precluded gathering information about the whole picture of the partner relationship and QOL issues. 
Men diagnosed with prostate cancer and meeting the inclusion criteria were included in the study. Data about time since diagnosis and type of treatment were gathered; however the study did not limit the inclusion to those with a specific treatment option or duration since diagnosis. Therefore, the results may not be generalized to all men diagnosed and treated for prostate cancer. The diverse population and small sample within each group size does not allow statistical analysis to compare QOL in men with different treatment options and those with different time since diagnosis.

Finally, the surveys were administered at the participating urology clinics during the wait time before a physician's appointment. So there were some cases in which there multiple interruptions by telephone calls or questions asked by clinic staff. Also, the participants might have been eager to complete the survey so as not to miss their appointment. Luckily, many of these clinics had a waiting time that was suitable for the length of the survey, and there were no major complaints about the length of the survey and its interruption of clinic appointments.

\section{Implications for Nursing Practice}

Prostate cancer prevalence is increasing and the positive disease outcomes allow men to live longer. However, these men will have to recognize that their life will change due to living with potential short term or long term sexual and urinary dysfunctions. As such, they will have to develop coping strategies in line with their socially constructed masculine norms and partner relations.

In Miami, the majority of the population is of Latin American and Caribbean origins. This is a unique population that has not been adequately represented in the literature. Their perceptions of prostate cancer treatment side effects and how the QOL is 
impacted was not very well understood. Research has highlighted the concepts of machismo and hypermasculinity in the Hispanic and Latin American cultures. As a result, it was hypothesized that the perceptions of men in Miami regarding their QOL in light of prostate cancer would be affected by the treatment side effects, masculinity, and partner relationships. However, the findings of this study did not support the proposed hypothesis. Sexual dysfunction and masculine norms did not affect these QOL of these men. The perceived partner relationship was the main variable that affected men's QOL. Nevertheless, the importance of sexual dysfunction and masculine norms could not be underestimated since they appeared to have an influence on different features of these men's lives. They might not have been major concepts that affected QOL, but they were related to social functioning and partner relationship which would ultimately indirectly affect QOL.

It is important for health care professionals and nurses to recognize how men perceive what is important for their QOL. Understanding what is the main contributor to QOL allows nurses to develop better educational programs to support men's knowledge and concerns about the disease, treatment process, and life after the cancer. Additionally, the findings of this study emphasize the importance of including the female partner as a major team player in the treatment of men with prostate cancer. Including interventions that strengthen and support the partner relationship would strengthen men's support system and therefore would enhance their QOL. Finally, accounting for cultural and social variables is important to make the treatment and interventions acceptable to patients and their partners. These findings set the foundation for future research that would assist in developing the best practice to enhance the QOL of men. 


\section{Implications for Future Research}

Findings from this study provide an understanding of the perceived factors that influence the QOL in a sample of older men diagnosed with prostate cancer and from a multiethnic and multiracial sample. The findings of this study could be used to further explore the QOL of these men. Men in this study reported sexual functioning as being a problem that was not associated with masculine norms or did not affect their relationship satisfaction or QOL. Future research is needed to further understand the beliefs of older men regarding sexual practices especially since they were utilizing sexual enhancement regimens. As such, they are sexually active but the importance of maintaining their sexual functioning and how much it would affect their QOL is important to understand to be able to understand treatment and counseling strategies.

South Florida provides a unique diverse population. Studies planned to examine the differences in the contributions to QOL in men with prostate cancer from different ethnic and racial groups is important. Such studies will help understand the cultural differences among the major minority groups in South Florida and consequently will help inform health care practices. Having the majority of the sample born outside the United States, it would be interesting to assess if acculturation has an influence on masculinity, relationship satisfaction, or QOL.

South Florida is also known to be a retirement destination, and the older age population is increasing. It would be interesting to compare the major study variables among different age groups of men older than 65 years. There may be cohort differences that may need to be considered or accounted for especially that the American Urologic Association advises prostate cancer screening to stop after the age of 75 years. Also, 
with older men, do the concepts of masculinity and machismo change or become less rigid? It is important to understand how these concepts develop with older age and the diagnosis of prostate cancer.

Hispanic partnered men were recruited for this study. Men's conformity to the concepts of machismo were anticipated, and therefore the influences of prostate cancer treatment side effects were explained in relation to the relationship with the partner and QOL. Findings of this study were somewhat different than what has been reported in the literature. Future research using mixed methodologies would help explain the relationship dynamics and progression with the diagnosis of prostate cancer and initiation of treatment side effects. Also, multiple method studies would highlight on the coping strategies of these men within their relationships and how they try to maintain their family role and machismo image. Additionally, including the female partner in the study will help understand Hispanic women's relationship expectations and coping strategies with their husband's diagnosis.

Baseline sexual and urinary functioning are essential considerations in research to understand the post treatment outcomes of prostate cancer treatment. Findings from this study showed that men were reluctant to provide baseline information about their sexual functioning. Baseline sexual data provided did not predict post treatment outcomes which was contrary to what has been reported in the literature. The current study did not assess the baseline urinary functioning of men. A mixed methods study would provide information about the relationship between baseline sexual and urinary functioning and post treatment outcomes to inform future research. It is important to understand the baseline information that would suggest post treatment functioning in order to better 
educate men about the treatment side effects. Also, future research is needed to examine the extent to which urinary dysfunction impacts the lives of men in relation to disruption to life activities and work in addition the financial burden of continence pads and other required modifications.

A prostate cancer treatment option is chosen based on the patient's preference and the individualized prognosis of each man. Surgery, radiation, hormonal therapy, or watchful waiting alone can be chosen. However, at times and due to recurrence of the disease or the manner to which the cancer responds, a secondary treatment might be used or multiple treatment methods might be indicated. In the current study, the item asking about treatment option did not clarify whether the answer indicated the current treatment or if there were multiple treatments or any previous treatment regimens. Future studies need to clarify the course of treatment options to better explain the treatment side effects.

The finding of this study suggested that many men did not participate in the choice of treatment nor were their concerns accounted for. Future studies are needed to examine if QOL differs among men who participate in treatment options and those who do not. Does an educational intervention prior to the choice of treatment impact QOL after the initiation of treatment?

\section{Summary}

Prostate cancer, the leading cause of cancer in men, has positive survival rates and constitutes a challenge with its side effects (Jemal et al., 2006; Jemal et al., 2010; Siegel et al., 2013). Studies have discussed the relationship between prostate cancer treatment side effects on each of masculinity (Burns \& Mahalik, 2006; Fergus, Grey, \& Fich; 2002), partner relationship (Galbraith, Pedro, Jaffe, \& Allen, 2008; Sanders et al., 2006), 
and QOL (Howlet et al., 2010; Monga, Kerrigan, Thornby, Monga, \& Zimmermann, 2005) alone. However, few studies have highlighted the relationship between prostate cancer treatment side effects (i.e., sexual dysfunction, urinary incontinence), masculinity, and relationship with the partner together on QOL in men. The purpose of this study was to examine relative contributions of age, ethnicity, sexual dysfunction, urinary incontinence, masculinity, and perception of the relationship with the partner on the quality of life in men diagnosed with prostate cancer. Data were collected using self administered questionnaires measuring demographic variables, sexual and urinary functioning (UCLA PCI), masculinity (CMNI), partner relationship (DAS), and QOL (SF-36). A total of 117 partnered heterosexual men diagnosed with prostate cancer were recruited from four clinics in Miami, Florida. Men were $67.47(S D=8.42)$ years old and identified themselves to be of Hispanic origin $(54.3 \%, n=63)$. Findings demonstrated that there was a moderate significant negative relationship between urinary and sexual functioning. There was a significant strong negative relationship between partner relationship and masculinity. Additionally, there was a weak negative relationship between DAS and QOL. Hierarchal multiple regression showed that partner relationship $(\beta=-.25, t(91)=-2.28, p=.03)$ significantly contributed to the overall QOL. These findings highlight the importance of the relationship satisfaction in the QOL of men with prostate cancer. Nursing interventions to enhance the QOL of these men should consider strengthening the relationship and involving the female partner as an active participant. 


\section{REFERENCES}

Abdel-Wahab, M., Reis, I. M., Wu, J., \& Duncan, R. (2009). Second primary cancer risk of radiation therapy after radical prostatectomy for prostate cancer: An analysis of SEER data. Urology, 74(4), 866-871. doi:10.1016/j.urology.2009.02.085

Abreu, J. M., Goodyear, R. K., Campos, A., \& Newcomb, M D. (2000). Ethnic belonging and traditional masculinity ideology among African Americans, European Americans, and Latinos. Psychology of Men \& Masculinity, 1, 75-86. doi:10.1037/1524-9220.1.2.75

Addis, M. E., \& Mahalik, J. R. (2003). Men, masculinity, and the contexts of helpseeking. American Psychologist, 58, 5-14.

Adolfsson, J., Rönström, L., Löwhagen, T., Carstensen, J., \& Hedlund, P. O. (1994). Deferred treatment of clinically localized low grade prostate cancer: the experience from a prospective series at the Karolinska Hospital. The Journal of Urology, 152, 1757-1760.

Adolfsson, J., Steineck, G., \& Hedlund, P. O. (1997). Deferred treatment of clinically localized low-grade prostate cancer projected 15-year follow-up of the Karolinska series. Urology, 50(5), 722-726. doi:10.1016/S0090-4295(97)00320-8

Albertsen, P. C., Hanley, J. A., \& Fine, J. (2005). Conservative management of clinically localized prostate cancer. Journal of the American Medical Association, 293(17), 2095-2101. doi:10.1001/jama.293.17.2095

Alemozaffar, M., Regan, M. M., Cooperberg, M. R., Wei, J.T., Michalski, J.M., Sandler, H.M., . . . Sanda, M. G. (2011). Prediction of erectile function following treatment for prostate cancer. Journal of the American Medical Association, 306(11), 1205-1214. doi:10.1001/jama.2011.1333

Altekruse, S. F., Kosary, C. L., Krapcho, M., Neyman, N., Aminou, R., Waldron, W., .. . Edwards, B. K. (Eds.) (2010). SEER Cancer Statistics Review, 1975-2007. Retrieved from http://seer.cancer.gov/csr/1975_2007/

American Cancer Society. (2010). Cancer registry. Retrieved from www.cancer.org/acs/groups/content/@epidemiologysurveillance/documents/docu ment/acspc-026238.pdf

American Cancer Society. (2011). American Cancer Society cancer facts \& statistics, 2011. Retrieved from http://www.cancer.org/acs/groups/content/@epidemiologysurveilance/documents/ document/acspc-029771.pdf 
American Cancer Society [ACS]. (2013). Cancer facts \& figures 2013. Retrieved from http://www.cancer.org/acs/groups/content/@epidemiologysurveilance/documents/ document/acspc-036845.pdf

American Cancer Society [ACS]. (2012). Cancer Facts \& Figures 2012. Retrieved from

http://www.cancer.org/acs/groups/content/@epidemiologysurveilance/documents/ document/acspc-031941.pdf

Anastasiadis, A. G., Sachdev, R., Salomon, L., Ghafar, M. A., Stisser, B. C., Shabsigh, R., \& Katz, A. E. (2003). Comparison of health related quality of life and prostate associated symptoms after primary and salvage cryotherapy for prostate cancer. Journal of Cancer Research and Clinical Oncology, 129(12), 676-682. doi:10.1007/s00432-003-0472-4

Anger, J. T., Maliski, S. L., Krupski, T. L., Kwan, L., Gore, J. L., Fink, A., . . Litwin, M. (2007). Outcomes in men denied access to a California public assistance program for prostate cancer. Public Health Reports, 122, 217-223.

Araujo, A. B., Mohr, B. A., \& McKinlay, J. B. (2004). Changes in sexual function in middle-aged and older men: Longitudinal data from the Massachusetts male aging study. Journal of the American Geriatrics Society, 52(9), 1502-1509. doi:10.1111/j.0002-8614.2004.52413.x

Arciniega, G. M., Anderson, T. C., Tovar-Blank, Z. G., \& Tracey, T. J. G. (2008). Toward a fuller conception of machismo: Development of a traditional machismo and caballerismo scale. Journal of Counseling Psychology, 55, 19-33. doi:10.1037/002-0167.55.1.19

Asbell, S. O., Martz, K. L., Shin, K. H., Sause, W. T., Doggett, R. L., Perez, C. A., \& Pilepich, M. V. (1998). Impact of surgical staging in evaluating the radiotherapeutic outcome in RTOG \#77-06, a phase III study for T1BN0M0 (A2) and T2N0M0 (B) prostate carcinoma. International Journal of Radiation Oncology, Biology, and Physics, 40(4), 769-782. doi:10.1016/S03603016(97)00926-7

Aus, G., Pileblad, E., \& Hugosson, J. (2002). Cryosurgical ablation of the prostate: 5year follow-up of a prospective study. European Urology, 42(2), 133-138. doi:10.1016/S0302-2838(02)00261-0

Babington, L. M., Kelley, B. R., \& Patsdaughter, C. A. (2007). Risk behaviors of Dominican adolescents. Journal of Pediatric Health Care, 21(6), 372-380. doi:10.1016/j.pedhc.2007.03.005 
Babington, L. M., Kelley, B. R., \& Patsdaughter, C. A. (2009). Self-esteem and risk behaviors of Dominican adolescents. Issues in Comprehensive Pediatric Nursing, 32, 131-144. doi:10:1080/01460903062774

Badr, H. \& Carmack Taylor, C. L. (2009). Sexual dysfunction and spousal communication in couples with prostate cancer. Psycho-oncology, 110, 10021010. doi:10.1002/pon.1449

Banthia, R., Malcarne, V. L., Varni, J. W., Ko, C. M., Sadler, G. R., \& Greenbergs, H. L. (2003). The effects of dyadic strength and coping styles on psychological distress in couples faced with prostate cancer. Journal of Behavioral Medicine, 26(1), 3152. doi:10.01607715/03/0200-0031/0

Barbash, G. I., \& Glied, S. A. (2010). New technology and healthcare costs--the case of robotic assisted surgery. The New England Journal of Medicine, 363(8), 701704. doi:10.1056/NEJMp1006602

Basaria, S., Lieb, J., Tang, A. M., DeWeese, T., Carducci, M., Eisenberger, M., \& Dobs, A. S. (2002). Long-term effects of androgen deprivation therapy in prostate cancer patients. Clinical Endocrinology, 56(6), 779-786. doi:10.1046/j.13652265.2002.01551.x

Bennett, J. A., \& Riegal, B. (2003). United States Spanish short form 36 health survey: Scaling assumptions and reliability in elderly community dwelling Mexican Americans. Nursing Research, 52(4), 262-269.

Bergman, J., Saigal, C. S., Kwan, L., \& Litwin, M. (2010). Responsiveness of the University of California- Los Angeles Prostate Cancer Index. Urology, 75(6), 1418-1423. doi:10.1016/j.urology.2009.04.070

Bill-Avelson, A., Holmberg, L., Filén, F., Ruutu, M., Garmo, H., Busch, C., .. . Johansson, J. E. (2008). Radical prostatectomy versus watchful waiting in localized prostate cancer: The Scandinavian prostate cancer group-4 randomized trial. Journal of the National Cancer Institute, 100(16), 1144-1154. doi:10.1093/jnci/djn255

Bill-Axelson, A., Holmberg, L., Ruutu, M., Andersson, S.O., Bratell, S., Spångberg, A., . .. Johansson, J. E. (2005). Radical prostatectomy versus watchful waiting in early prostate cancer. New England Journal of Medicine, 352(19), 1977-1984. doi/full/10.1056/NEJMoa043739

Bill-Axelson, A., Holmberg, L., Filen, F., Ruutu, M., Garmo, H., Busch, C., , . . Scandinavian Prostate Cancer Group Study Number 4. (2008). Radical prostatectomy versus watchful waiting in localized prostate cancer: The 
Scandinavian Prostate Cancer Group 4 randomized trial. Journal of the National Cancer Institute, 100(16), 1144-1154. doi:10.1093/jnci/djn225

Bishoff, J. T., Motley, G., Optenberg, S. A, Stein, C. R., Moon, K. A., Browning, S. M., . .. Thompson, I. M. (1998). Incidence of fecal and urinary incontinence following radical perineal and retropubic prostatectomy in a national population. The Journal of Urology, 160(2), 454-458. doi:10.1016/S0022-5347(01)62924-0

Blank, T. (2005). Gay men and prostate cancer: Invisible diversity. Journal of Clinical Oncology, 23(12), 2593-2596. doi:10.1200/JCO.2005.00.968

Boehmer, U., \& Clark, J. A. (2001). Married couples' perspectives on prostate cancer diagnosis and treatment decision making. Psychooncology, 10(2), 147-155. doi:10.1002/pon.504

Bokhour, B. G., Clark, J. A., Inui, T. S., Silliman, R. A., \& Talcott, J. A. (2001). Sexuality after the treatment for early prostate cancer: Exploring the meanings of "erectile dysfunction". Journal of Geriatrics Internal Medicine. 16(10), 649-655. doi:10.1111/j.1525-1497.2001.00832.x

Bradby, H. (2003). Describing ethnicity in health research. Ethnicity \& Health, 8(1), 513. doi: $10.1080 / 13557850303555$

Bradley, C. J., Yabroff, K. R., Dahman, B., Feuer, E. J., Mariotto, A., \& Brown, M. L. (2008). Productivity costs of cancer mortality in the United States: 2000-2020. Journal of National Cancer Institute, 100, 1763-1770. doi:10.1093/jnci/djn384

Brandeis, J. M., Litwin, M. S., Burnison, M., \& Reiter, R. E. (2000). Quality of life outcomes after brachytherapy or early stage prostate cancer. The Journal of Urology, 163(3), 851-857. doi:10.1016/S0022-5347(05)67818-4

Burns, S. M., \& Mahalik, J. R. (2006). Physical health, self reliance, and emotional control as moderators of the relationship between locus of control and mental health among men treated for prostate cancer. Journal of Behavioral Medicine, 29(6), 561-572. doi:10.1007/s10865-006-9076-1

Burns, S. M. \& Mahalik, J. R. (2007). Understanding how masculine gender scripts may contribute to men's adjustment following treatment for prostate cancer. American Journal of Men's Health, 1(4), 250-261. doi:10.1177/1557988306293380

Burns, S. M., \& Mahalik, J. R. (2008). Sexual functioning as a moderator of the relationships between masculinity and men's adjustment following treatment for prostate cancer. American Journal of Men's Health, 2(1), 6-16. doi:10.1177/1557988307304325 
Catalona, W. J, \& Basler, J. W. (1993). Return of erections and urinary continence following nerve sparing radical retropubic prostatectomy. The Journal of Urology, 150(3), 905-907.

Catalona, W. J., \& Bigg, S. W. (1990). Nerve-sparing radical prostatectomy: Evaluation of results after 250 patients. The Journal of Urology, 143 (3), 538-543.

Cella, D. F. (1994). Quality of life: Concepts and definitions. Journal of Pain and Symptom Management, 9, 186-192. doi:10.1016/0885-3924(94)90129-5

Centers for Medicare \& Medicaid Services [CMS]. (2005). Medicare National Coverage Determinations Manual. Retrieved from https://www.cms.gov/manuals/downloads/ncd103c1_Part2.pdf

Chan, E. C. Y., McFall, S. L., Byrd, T. L., Mullen, P. D., Volk, R. J., Ureda, J., . . . Bartholomew, L. K. (2011). A community-based intervention to promote informed decision making for prostate cancer screening among Hispanic American men changed knowledge and role preferences: A cluster RCT. Patient Education and Counseling, 84(2), e44-51.

Charmaz, K. (1995). Identity dilemmas of chronically ill men. In D. Sabo \& D. F. Gordon. Men's health \& illness: Gender, power, and the body (pp. 266-291). Thousand Oaks, CA: Sage.

Chapple, A., \& Ziebland, S. (2002). Prostate cancer: Embodied experience and perceptions of masculinity. Sociology of Health and Illness, 24(6), 820-841. doi:10.1111/1467-9566.00320

Chen, R. C., Clark, J. A., \& Talcott, J. A. (2009). Individualizing quality of life outcomes reporting: How localized prostate cancer treatments affect patients with different levels of baseline urinary, bowel, and sexual function. Journal of Clinical Oncology, 27(24), 3916-1922. doi:10.1200/JCO.2008.18.6486

Cheng, C. (2008). Marginalized masculinities and hegemonic masculinity: An introduction. Journal of Men's Studies, 7(3), 295-315. doi:10.3149/jms.0703.295

Chida, Y., \& Steptoe, A. (2008). Positive psychological well-being and mortality: A quantitative review of prospective observational studies. Psychosomatic Medicine, 70(7), 741-756. doi:10.1097/PSY.0b013e31818105ba

Chodak, G. W., Thisted, R. A., Gerber, G. S., Johansson, J. E., Adolfsson, J., Jones, G. W., . . Warner, J. (1994). Results of conservative management of clinically localized prostate cancer. New England Journal of Medicine, 330(4), 242-248. doi:10.1056/NEJM199401273300403 
Chodak, J. A., \& Warren, K. S. (2006). Watchful waiting for prostate cancer: A review article. Prostate Cancer and Prostatic Disease, 9, 25-29.

doi:10.1038/sj.pcan.4500857

Choo, R., Long, J., Gray, R., Morton, G., Gardner, S., \& Danjoux, C. (2010). Prospective survey of sexual function among patients with clinically localized prostate cancer referred for definitive radiotherapy and the impact of radiotherapy on sexual function. Supportive Care in Cancer, 18(6), 715-722. doi:10.1007/s00520-009-0675-6

Cialdini, R. B., \& Trost, M. R. (1999). Social influence: Social norms, conformity, and compliance. In D. Gilbert, S. Fiske, \& G. Lindzy (Eds.), The handbook of social psychology (vol. 2; pp.151-192). Boston, MA: McGraw-Hill.

Clarke, J. N. (1999). Prostate cancer's hegemonic masculinity in selected print mass media depictions (1974-1995). Health Communication, 11(1), 59-74. doi:10.1207/s15327027hc1101_4

Cohen, J. (1988). Statistical power analysis for the behavioral sciences (2nd ed.). Hillsdale, NJ: Erlbaum.

Cohen, J., Cohen, P., West, S. G., \& Aiken, L. S. (2003). Applied multiple regression/correlation analysis for the behavioral sciences (3rd ed.). Mahwah, NJ: Erlbaum.

Connell, R. (2010). Masculinities. Oxford, England: Polity.

Connell, R. (1995). Masculinities. Oxford, England: Polity.

Corral, D. A, \& Bahnson, R. R. (1994). Survival of men with clinically localized prostate cancer detected in the eighth decade of life. The Journal of Urology, 151(5), 1326-1329.

Courtney, W. H. (2000). Engendering health: A social constructionist examination of men's health beliefs and behaviors. Psychology of Men and Masculinity, 1, 4-15. doi:10.1037/1524-9220.1.1.4

Crook, J. M., Gomez-Iturriaga, A., Wallace, K., Ma, C., Fung, S., Alinhai, S., . . Fleshner, N. (2011). Comparison of health-related quality of life 5 years after SPIRIT: Surgical prostatectomy versus interstitial radiation intervention trial. Journal of Clinical Oncology, 29(4), 362-368. doi:10.1200/JCO.2010.31.7305

D'Amico, A. V., Denham, J. W., Crook, J., Chen, M. H., Goldhaber, S. Z., Lamb, D. S., . . Kantoff, P. W. (2007). Influence of androgen suppression therapy for prostate 
cancer on the frequency and timing of fatal myocardial infarctions. Journal of Clinical Oncology, 25(17), 2420-2425. doi:10.1200/JCO.2006.09.3369

D’Amico, A. V., Manola, J., Loffredo, M., Renshaw, A. A., DellaCroce, A., \& Kantoff, P. W. (2004). 6-month androgen suppression plus radiation therapy vs. radiation therapy alone for patients with clinically localized prostate cancer: A randomized control trial. Journal of American Medical Association, 292, 821-827. doi:10.1001/jama.292.7.821

DiBlasio, C. J., Malcolm, J. B., Derweesh, I. H., Womack, J. H., Kincade, M. C., Mancini, J. G., ... Wake, R. W. (2008). Patterns of sexual and erectile dysfunction and response to treatment in patients receiving androgen deprivation therapy for prostate cancer. British Journal of Urology International, 102(1), 3943. doi:10.1111/j.1464-410X.2008.07505.x

Daniell, H. W. (1997). Osteoporosis after orchiectomy for prostate cancer. The Journal of Urology, 157(2), 439-444. doi:10.1016/S0022-5347(01)65165-6

DeLamater, J., \& Moorman, S. (2007). Sexual behavior in later life. Journal of Aging and Health, 19(6), 921-945. doi:10.1177/0898264307308342

Donnelly, B. J., Saliken, J. C., Ernst, D. S., Ali-Ridha, N., Brasher, P. M., Robinson, J. W., \& Rewcastle, J. C. (2002). Prospective trial of cryosurgical ablation of the prostate: Five-year results. Urology, 60(4), 645-649. doi:10.1016/S00904295(02)01839-3

Drevdahl, D., Taylor, J., \& Phillips, D. A. (2001). Race and ethnicity as variables in nursing research, 1952- 2000. Nursing Research, 50(5), 305-313.

Duncan, D. \& Dowseit, G. W. (2010). "There's no teleology to it; it's just about the spirit of play": Men, intimacy, and "late" modernity. Journal of Men's Studies, 18(1), 45-62. doi:10.3149/jms.1801.45.

Duncan, W., Warde, P., Catton, C. N., Munro, A. J., Lakier, R., Gadalla, T., \& Gospodarowicz, M. K. (1993). Carcinoma of the prostate: Results of radical radiotherapy (1970-1985). International Journal of Radiation Oncology, Biology, and Physics, 26(2), 203-110.

Edwards, B. K., Ward, E., Kohler, B. A., Eheman, C., Zauber, A. G., Aderson, R. N., . . . Ries, L. A. (2010). Annual report to the nation on the status of cancer, 19752006, featuring colorectal cancer trends and impact interventions (risk factors, screening, and treatment) to reduce future rates. Cancer, 116, 544-573. doi:10.1002/cncr.24760 
Espey, D. K., Wu, X. C., Swan J., Wiggins, C., Jim, M. A., Ward, E., . . Edwards, B. K. (2007). Annual report to the nation on the status of cancer, 1975-2004, featuring cancer in American Indians and Alaska Natives. Cancer, 110, 2119-2152. doi:10.1002/cncr.23044

Fair, W. R., Cookson, M. S., Stroumbakis, N., Cohen, D., Aprikian, A. G., Wang, Y., .. . Reuter, V. E. (1997). The indications, rationale, and results of neoadjuvant androgen deprivation in the treatment of prostatic cancer: Memorial SloanKettering Cancer Center results. Urology 49, 46-55. doi:10.1016/S00904295(97)00169-6,

Farrell, W. (1993). The myth of male power. New York City, NY: Simon \& Schuster.

Farwell, W. R., Linder, J. A., \& Jha, A. K. (2007). Trends in prostate-specific antigen testing from 1995 through 2004. Archives of Internal Medicine, 167, 2497-2502. doi:10.1001/archinte.167.22.2497

Faul, F., Erdfelder, E., Buchner, A., \& Lang, A. G. (2009). Statistical power analyses using G*Power 3.1: Tests for correlation and regression analyses. Behavior Research Methods, 41(4), 1149-1160. doi:10.3758/BRM.41.4.1149

Faul, F., Erdfelder, E., Lang, A. G., \& Buchner, A. (2007). G*Power 3: A flexible statistical power analysis program for the social, behavioral, and biomedical sciences. Behavior Research Methods, 39, 175-191. doi:10.3758/BF03193146

Ferrans, C. E., Zerwic, J. J., Wilbur, J. E., \& Larson, J. L. (2005). Conceptual model of health related quality of life. Journal of Nursing Scholarship, 37(4), 336-342. doi:10.1111/j.1547-5069.2005.00058.x

Fergus, K. D., Gray, R. E., \& Fitch, M. (2002). Sexual dysfunction and the preservation of manhood: Experiences of men with prostate cancer. Journal of Health Psychology, 7, 303-316. doi:10.1177/1359105302007003223

Forman, J. D., Order, S. E., Zinreich, E. S., Lee, D. J., \& Wharam, M. D. (1986). Carcinoma of the prostate in the elderly: The therapeutic ratio of definitive radiotherapy. The Journal of Urology, 136(6), 1238-1241.

Fowler, F. J., Barry, M. J., Lu-Yao, G., Wasson, J. H., \& Bin, L. (1996). Outcomes of external-beam radiation therapy for prostate cancer: A study of Medicare beneficiaries in three surveillance, epidemiology, and end result areas. Journal of Clinical Oncology, 14, 2258-2265.

Fowler, F. J. Jr, McNaughton Collins, M., Walker Corkery, E., Elliott, D. B., \& Barry, M. J. (2002). The impact of androgen deprivation on quality of life after radical 
prostatectomy for prostate carcinoma. Cancer, 95(2), 287-295.

doi:10.1002/cncr.10656

Galbraith, M. E., \& Crighton, F. (2008). Alterations of sexual function in men with cancer. Seminars in Oncology Nursing, 24(2), 102-114.

doi:10.1016/j.soncn.2008.02.010

Galbraith, M. E., Pedro, L. W., Jaffe, A. R., \& Allen, T. L. (2008). Describing healthrelated quality of life outcomes for couples experiencing prostate cancer:

Differences and similarities. Oncology Nursing Forum, 35, 794-801. doi:10.1188/08.ONF.794-801

Giarelli, E., McCorkle, R., \& Monturo, C. (2003). Caring for a spouse after prostate surgery: The preparedness needs of wives. Journal of Family Nursing, 9, 453485. doi:10.1177/1074840703258317

Gilbert, E., Ussher, J. M., \& Hawkins, Y. (2009). Accounts of disruption to sexuality following cancer: The perspective of informal carers who are partners of a person with cancer. Health, 13, 523-541. doi:10.1177/1363459308336795

Gontero, P., Galzerano, M., Bartoletti, R., Magnanib, C., Tizzania, A., Fread , B., \& Mondainic, N. (2007). New insights into the pathogenesis of penile shortening after radical prostatectomy and the role of postoperative sexual function. The Journal of Urology, 178(2), 602-607. doi:10.1016/j.juro.2007.03.119

Gralnek, D., Wessels, H., Cui, H., \& Dalkin, B. L. (2000). Differences in sexual function and quality of life after nerve sparing and nonnerve sparing radical retropubic prostatectomy. The Journal of Urology, 163(4), 1166-1170. doi:10.1016/S00225347(05)67715-4

Gray, R. E., Fitch, M., Phillips, C., Labrecque, M., \& Fergus, K. (2000). Managing the impact of illness: The experiences of men with prostate cancer and their spouses. Journal of Health Psychology, 5(4), 531-548. doi:10.1177/135910530000500410

Gray, R. E., Fitch, M. I., Fergus, K. D., Mykhalovskiy, E., \& Church, K. (2002). Hegemonic masculinity and the experience of prostate cancer: A narrative approach. Journal of Aging and Identity, 7(1), 43-62. doi:1087-3732/020/03000043/0

Greenspan, G. L., Coates, P., Sereika, S. M., Nelson, J. B., Trump, D. L., \& Resnick, N. M. (2005). Bone loss after initiation of androgen deprivation therapy in patients with prostate cancer. The Journal of Clinical Endocrinology \& Metabolism, 90(12), 6410-6417. doi:10.1210/jc.2005-0183 
Grover, S. A., Zowall, H., Coupal, L., \& Krahn, M. D. (1999). Prostate cancer: The economic burden. Canadian Medical Association, 160(5), 685-690.

Grunfed, E. A., Drudge-Coates, L., Rixon, L., Eaton, E., \& Cooper, A. F. (2013). "The only way I know how to live is to work: A qualitative study of work following treatment for prostate cancer. Health Psychology, 32, 1, 75-82. doi:10.1037/a0030387

Hamilton, A. S., Stanford, J. L., Gilliland, F. D., Albertsen, P. C., Stephenson, R. A., Hoffman, R. M., . . Potosky, A. L. (2001). Health outcomes after external-beam radiation therapy for clinically localized prostate cancer: Results from the Prostate Cancer Outcomes Study. Journal of Clinical Oncology, 19(9), 2517-2526.

Hanlon, A. L., Schultheiss, T. E., Hunt, M. A., Movsas, B., Peter, R. S., \& Hanks, G. E. (1997). Chronic rectal bleeding after high-dose conformal treatment of prostate cancer warrants modification of existing morbidity scales. International Journal of Radiation Oncology, Biology, and Physics, 38(1), 59-63.

Harden, J. K., Northouse, L., \& Mood, D. (2006). Qualitative analysis of couples' experience with prostate cancer by age cohort. Cancer Nursing, 29(5), 367-377.

Harden, J., Schafenacker, A., Northouse, L., Mood, D., Smith, D., Pienta, K., . . . Baranowski, K. (2002). Couples' experiences with prostate cancer: Focus group research. Oncology Nursing Forum, 29(4), 701-709. doi:10.1188/02.ONF.701709

Hawes, S. M., Malcarne, V. L., Ko, C. M., Sadler, G. R., Banthia, R., Sherman, S. A., . . Schmidt, J. (2006). Identifying problems faced by spouses and partners of patients with prostate cancer. Oncology Nursing Forum, 33(4), 807-814. doi:10.1188/06.ONF.807-814

Hawkins, Y., Ussher, J., Gilbert, E., Perz, J., Sandoval, M., \& Sundquist, K. (2009). Changes in sexuality and intimacy after the diagnosis and treatment of cancer. Cancer Nursing, 32, 271-280. doi:10.1097/NCC.0b013e31819b5a93

Hegarty, J. M., Wallace, M., \& Comber, H. (2008). Uncertainty and quality of life among men undergoing active surveillance for prostate cancer in the United States and Ireland. American Journal of Men's Health, 2(2), 133-142. doi:10.1177/1557988307300467

Howlett, K., Koetters, T., Edrington, J., West, C., Paul, S., Lee, K., . . Miakowski, C. (2010). Changes in sexual function on mood and quality of life in patients undergoing radiation therapy for prostate cancer. Oncology Nursing Forum, 37(1), E58-E66. doi:10.1188/10.ONF.E58-E66 
Hoyt, M. A., Stain, A. A., Irwin, M.R., \& Thomas, K. S. (2013). Cancer-related masculine threat, emotional approach coping, and physical functioning following treatment for prostate cancer. Health Psychology, 32, 1, 66-74. doi:10.1037/a0030020

Hoznek, A., Menard, Y., Salomon, L., \& Abboud, C. C. (2005). Update on laparoscopic and robotic radical prostatectomy. Current Opinion in Urology, 15, 173-180.

Incrocci, L., Koper, P. C., Hop, W. C., \& Slob, A. K. (2001). Sildenafil citrate (Viagra) and erectile dysfunction following external beam radiotherapy for prostate cancer: A randomized, double-blind, placebo-controlled, cross-over study. International Journal of Radiation Oncology, Biology, and Physics, 51(5), 1190-1195. doi:10.1016/S03603016(01)01767-9

Isajiw, W. W. (1974). Definitions of ethnicity. Ethnicity, 1(2), 111-124.

Institute for Clinical and Economic Review. (n.d.). Overview: Radical Prostatectomy. Retrieved from http://www.icer-review.org/index.php/View-document/57Radical-Prostatectomy-Overview.html?format=raw\&tmpl=component

Jemal, A., Clegg, I. X., Ward, E., Lynn A. G., Ries, L. A. G., Wu, X., . . .Edwards, B. K. (2004). Annual report to the nation on the status of cancer, 1975-2001, with special feature regarding survival. Cancer, 101, 3-27. doi:10.1002/cncr.20288

Jemal , A., Siegel, R., Ward, E., Murray, T., Xu, J., Smigal, C., \& Thun, M. J. (2006). Cancer statistics, 2006. Cancer Journal Clinicians, 56, 106-130. doi:10.3322/canjclin.56.2.106

Jemal, A., Siegel, R., Xu, J., \& Ward, E. (2010). Cancer statistics, 2010. CA: A Cancer Journal for Clinicians, 60, 277-300. doi:10.3322/caac.20073

Johansson, J. E., Andren, O., Andersson, S. O., Dickman, P. W., Holmberg, L., Magnusun, A., \& Adami, H. O. (2004). Natural history of early, localized prostate cancer. Journal of the American Medical Association, 291(22), 27132719. doi:10.1001/jama.291.22.2713

Johansson, J. E., Holmberg, L., Johansson, S., Bergstrom, R., \& Adami, H. O. (1997). Fifteen-year survival in prostate cancer: A prospective population-based study in Sweden. Journal of the American Medical Association, 277(6), 467-471. doi:10.1001/jama.1997.03540300035030

Johnson, J. R., \& Temple, R. (1985). Food and Drug Administration requirements for approval of new anticancer therapies. Cancer Reports, 69, 1155-1157. 
Jones, S. G., Patsdaughter, C. A., \& Martinez Cardenas, V. M. (2011). Lessons learned from the Viagra study: Methodological challenges in recruitment of older and minority heterosexual men for research on sexual practices and risk behaviors. Journal of the Association of Nurses in AIDS Care, 22(4), 320-329. doi:10.1016/j.jana.2010.10.009

Jones, R., Richard, S., \& Ishan, W. (2009). Strategies for recruiting African American men into prostate cancer screening studies. Nursing Research, 58(6), 452-456. doi:10.1097/NNR.0b013e3181b4bade

Jones, R. A., Steeves, R., \& Williams, I. (2010). How African American men decide whether or not to get prostate screening. Cancer Nursing, 32(2), 166-172. doi:10.1097/NCC.0b013e3181982c6e

Jones, R. A., Wenzel, J., Hinton, I., Cary, M., Jones, N. R., Krumm, S., \& Ford, J. G. (2011). Exploring cancer support needs for older African American men with prostate cancer. Supportive Care in Cancer, 19(9), 1411-1419. doi:10.1007/s00520-010-0967-x

Joseph, J. V., Vincente, I., Madeb, R., \& Patel, H. R.. H. (2005). Robotic-assisted vs laparoscopic radical prostatectomy: Are there any differences? British Journal of Urology International, 96(1), 39-42. doi:10.1111/j.1464-410X.2005.05563.x

Kaasa, S., Mastekaasa, A., \& Lund, E. (2003). Prognostic factors for patients with inoperable non-small cell lung cancer, limited disease: The importance of patients' subjective experience of disease and psychosocial well-being. Radiotherapy and Oncology, 15(3), 235-242. doi:10.1016/0167-8140(89)90091-1

Keating, N. L., O'Malley, A. J., Freedland, S. J., \& Smith, M. R. (2010). Diabetes and cardiovascular disease during androgen deprivation therapy: Observational study of veterans with prostate cancer. Journal of the National Cancer Institute, 102(1), 39-46. doi:10.1093/jnci/djp404

Keating, N. L., O'Malley, A. J., \& Smith ,M. R. (2006). Diabetes and cardiovascular disease during androgen deprivation therapy for prostate cancer. Journal of Clinical Oncology, 24(27), 4448-4456. doi:10.1200/JCO.2006.06.2497

Kelly, D. (2009). Changed men: The embodied impact of prostate cancer. Qualitative Health Research, 19(2), 151-163. doi:10.1177/1049732308328067

Kershaw, T. S., Mood, D. W., Newth, G., Ronis, D. L., Sanda, M. G., Vaishampayan, U., $\&$ Northouse, L. L. (2008). Longitudinal analysis of a model to predict quality of life in prostate cancer patients and their spouses. Annals of Behavioral Medicine, 36(2), 117-128. doi:10.1007/s12160-008-9058-3 
Khan, S. I., Hudson-Rodd, N., Saggers, S., Bhuiyan, M. I., Bhuiya, A., Karim, S. A., \& Rauyajin, O. (2008). Phallus, performance and power: Crisis of masculinity. Sexual and Relationship Therapy, 23, 37-49. doi:10.1080/14681990701790635

Kimmel, M. (2000). The gendered society. London, England: Oxford University Press.

Kimmel, M. (1990). After fifteen years: The impact of the sociology of masculinity on the masculinity of sociology. London, England: Unwin Hyman.

Kirschenbaum, A. (1995). Management of hormonal treatment effects. Cancer ,75, 1983-1986. doi:10.1002/1097-0142(19950401)75:7+<1983

Kohli, M., \& Tindall, D. (2010). New developments in the medical management of prostate cancer. Mayo Clinic Proceedings, 85(1), 77-86. doi:10.4065/mcp.2009.0442

Koltz, L. H., Goldenberg, S. L., Jewett, M. A., Fradet, Y., Nam, R., Barkin, J., . . . Chatterjeem, S. (2003). Canadian Uro-Oncology Group. Long term follow up of a randomized trial of 0 versus 3 months of neoadjuvant androgen ablation before radical prostatectomy. The Journal of Urology, 170, 791-794. doi:10.1097/01.ju.0000081404.98273.fd

Korfage, I. J., Hak, T., de Koning, H. J., \& Essink-Bot, M. L. (2006). Patients' perceptions of the side effects of prostate cancer treatment: A qualitative interview study. Social Science \& Medicine, 63(4), 911-919. doi:10.1016/j.socscimed.2006.01.027

Krongrad, A., Perczek, R. E., Burke, M. A., Granville, L. J., Lai, H., \& Lai, S. (1997). Reliability of Spanish translations of select urologic quality of life instruments. The Journal of Urology, 158(2), 493-496. doi:10.1016/S0022-5347(01)64512-9

Langenhuijsen, J. F., Broers, E. M. P., \& Vergunst, H. (2009). Cryosurgery for prostate cancer: An update on clinical results of modern cryotechnology. European Association of Urology, 55, 76-86. doi:10.1016/j.eururo.2008.08.063

Laumann, E. O., Glasser, D. B., Moreira, E. D., \& GSSAB (Global Study of Sexual Attitudes and Behaviors) Investigators' Group. (2009). A population-based survey of sexual activity, sexual problems and associated help-seeking behavior patterns in mature adults in the United States of America. Journal of Impotence Research, 21(3), 171-178. doi:10.1038/ijir.2009.7

Lavery, J. F., \& Clarke, V. A. (1999). Prostate cancer: Patients' and spouses' coping and marital adjustment. Psychology, Health, \& Medicine, 4(3), 289-302. doi:13548506/99/030289-14 
Lepor, H., Kaci, L., and Xue, X. (2004). Continence following radical retropubic prostactomy using self-reporting instruments. The Journal of Urology 171(3), 1212-1215. doi:10.1097/01.ju.0000110631.81774.9c

Levine, G. N., D'Amico, A. V., Berger, P., Clark, P. E., Eckel, R. H., Keating, N. L., . . Zakai, N. (2010). Androgen-deprivation therapy in prostate cancer and cardiovascular risk: A science advisory from the American Heart Association, American Cancer Society, and American Urological Association: endorsed by the American Society for Radiation Oncology. CA: A Cancer Journal for Clinicians, 60(3), 194-201. doi:10.3322/caac.20061

Lim, A. J., Brandon, A. H., Fiedler, J., Brickmana, A. L., Boyera, C. I., Raub, W. A., \& Soloway, M. S. (1995). Quality of life: radical prostatectomy versus radiation therapy for prostate cancer. The Journal of Urology, 154(4), 1420-1425. doi:10.1016/S0022-5347(01)66881-2

Lindau, S. T., Schumm, P., Laumann, E. O., Levinson, W., O’Muircheartaigh, C. A. \& Waite, L. J. (2007). A study of sexuality and health among older adults in the United States. New England Journal of Medicine, 357, 762-774. doi:10.1056/NEJMoa067423

Linkenbach, J., \& Perkins, H. W. (2006). Montana's MOST of US Don't Drink and Drive Campaign: A social norms strategy to reduce impaired driving among 21- 34 year olds. National Highway Traffic Safety Administration (NHTSA) Publication: DOS HS 809869.

Litwin, M., \& McGuigan, K. A. (1999). Accuracy of recall in health related quality of life assessment among men treated for prostate cancer. Journal of Clinical Oncology, 17(9), 1882-2888.

Litwin, M. S., Hays, R. D., Fink, A., Ganz, P. A., Leake, B., \& Brook, R. H. (1998). The UCLA Prostate Cancer Index: Development, reliability, and validity of a health related quality of life measure. Medical Care, 36(7), 1002-1012.

Litwin, M. S., Hays, R. D., Fink, A., Ganz, P. A., Leake, B., Leach, G. E., \& Brook, R. H. (1995). Quality-of-life outcomes in men treated for localized prostate cancer. Journal of American Medical Association, 273(2), 129-135. doi:10.1001/jama.1995.03520260051032

Lodding, P., Bergdahl, C., Nyberg, M., Pileblad, E., Stranne, J., \& Hugosson, J. (2001). Inguinal hernia after radical retropubic prostatectomy for prostate cancer: A study of incidence and risk factors in comparison to no operation and lymphadenectomy. The Journal of Urology, 166(3), 964-967. doi:10.1016/S0022-5347(05)65874-0 
Loizides, E., Swierzewski. M. J., O’Neill, C., Griesser, J., \& Smith, T. (2004). Early response time in sexual activity and mood following testosterone gel replacement in hypogonadal males from the Testim START study. Reviews in Urology, 6, S16-S21.

Lu-Yao, G. L., Albertsen, P. C., Moore, D. F., Shih, W., Lon, Y., DiPaola, R. S., Barry, M. J., ... Yao, S. L. (2009). Outcomes of localized prostate cancer following conservative management. Journal of the American Medical Association, 302(11), 1202-1209. doi:10.1001/jama.2009.1348

Lughezzani, G., Sun, M., Perrotte, P., Alasker, A., Jeldres, C., Isbarn, H., . . . Karakiewicz, P. I. (2010). Comparative study of inguinal hernia repair rates after radical prostatectomy or external beam radiotherapy. International Journal of Radiation Oncology, Biology, and Physics, 78(5), 1307-1313. doi:10.1016/j.ijrobp.2009.09.065

Lugo, A. (1990). Cultural production and reproduction in Ciudad Juarez Mexico: Tropes at play amog maquiladora workers. Cultural Anthropology, 5, 173-196. doi:10.1525/can.1990.5.2.02a00030

Lusher, D., \& Robins, G. (2010). A social network analysis of hegemonic and other masculities. Journal of Men's Studies, 16(1), 22-44. doi:10.31.49/jms.1801.22

Mahalik, J. R., Burns, S. M., \& Syzdek, M. (2007). Masculinity and perceived normative health behaviors as predictors of men's health behaviors. Social Science \& Medicine, 64, 2201-2209. doi:10.1016/jsoccimed.2007.02.035.

Mahalik, J. R., Locke, B. D., Ludlow, L. H., Diemer, M. A., Scott, R. P. J., Gottfried, M., \& Freitas, G. (2003). Development of the Conformity to Masculine Norms Inventory. Psychology of Men and Masculinity, 4(1), 3-25. doi:10.1037/15249220.4.1.3

Maliski, S. L., Rivera, S., Connor, S., Lopez, G., \& Litwin, M. S. (2008). Renegotiating masculine identity after prostate cancer treatment. Qualitative Health Research, 18(12), 1609-1620. doi:10.1177/1049732308326813

Mariotto, A. B., Yabroff, K. R., Shao, Y., Feuer, E. J., \& Brown, M. L. (2010). Projections of the cost of cancer care in the United States: 2010-2020. Journal of National Cancer Institute, 103(2), 117-128. doi:10.1093/jnci/djq495

Mazur, A., \& Booth, A. (1998). Testosterone and dominance in men. Behavioral and Brain Science, 21, 353-397. 
McCullough, A. (2008). Penile change following radical prostatectomy: Size, smooth muscle atrophy, and curve. Current Urology Reports, 9(6), 492-499.

doi:10.1007/s11934-008-0084-2

McCabe, M. P., \& Matic, H. (2008). Erectile dysfunction and relationships: Views of men with erectile dysfunction and their partners. Sexual and Relationship Therapy, 23, 51-60. doi:10.1080/14681990701705559

McVittie, C., \& Willock, J. (2006). "You can't fight windmills": How older men do health, ill health, and masculinities. Qualitative Health Research, 16(6), 788-801. doi: $10.1177 / 1049732306288453$

Mertler, C. A., \& Vannatta, R. A. (2013). Advanced and multivariate statistical methods: Practical application and interpretation ( $5^{\text {th }}$ ed.). Los Angeles, CA: Pyrzak.

Meyer, J. P., Gillatt, D. A., Lockyer, R., \& Macdonagh, R. (2003). The effect of erectile dysfunction on the quality of life of men after radical prostatectomy. British Journal of Urology International, 92, 929-931. doi:10.1046/j.1464410x.2003.04530.x

Mikhail, A. A., Stockton, B. R., Orvieto, M. A., Chien, G. W., Gong, E. M., Zorn, K. C., Brendler, C. B., . . Shalhav, A. L. (2006). Robotic assisted laparoscopic prostatectomy in overweight and obese patients. Urology, 67(4), 774-779. doi:10.1016/j.urology.2005.10.049

Milecki, P., Martenka, P., Antczak, A., \& Kwais, Z. (2010). Radiotherapy combined with hormonal therapy in prostate cancer: The state of the art. Cancer Management and Research, 2, 243-253. doi:10.2147/CMR.S8912

Mittan, D., Lee, S., Miller, E., Perez, R. C., Basler, J. W., \& Bruder, J. M. (2002). Bone loss following hypogonadism in men with prostate cancer treated with $\mathrm{GnRH}$ analogs. The Journal of Clinical Endocrinology \& Metabolism, 87(8), 36563661. doi:10.1210/jc.87.8.3656

Mohan, R., Beydoun, H., Barnes-Ely, M. L., Lee, L., Davis, J., Lance, R., \& Schellhammer, P. (2009). Patients' survival expectations before localized prostate cancer treatment by treatment status. Journal of American Board of Family Medicine, 22(3), 247-256. doi:10.3122/jabfm.2009.03.080200

Monga, U., Kerrigan, A. J., Thornby, J., Monga, T. N., \& Zimmermann, K. P. (2005). Longitudinal study of life in patients with localized prostate cancer undergoing radiotherapy. Journal of Rehabilitation Research \& Development, 42(3), 391400. doi:10.1682/JRRD.2004.06.0071 
Montazeri, A. (2009). Quality of life data as prognostic indicators of survival in cancer patients: An overview of the literature from 1982 to 2008. Health and Quality of Life Outcomes, 7(102). doi:10.1186/1477-7525-7-102. Retrieved from http://www.hqlo.com/content/7/1/102

Nagel, J. (1994). Constructing ethnicity: Creating and recreating ethnic identity and culture. Social Problems, 41(1), 152-176.

National Center for Chronic Disease Prevention and Health Promotion [CDC]. (2013). The state if aging \& health in America 2013. Retrieved from www.cdc.gov/aging/pdf/state-aging-health-in-america-2013.pdf

National Cancer Institute (NCI). (2011). DevCan: Probability of developing or dying of cancer software, version 6.6.0. Retrieved from www.surveillance.cancer.gov/devcan

National Cancer Institute [NCI]. (2013). General information about prostate cancer. Retrieved from http://www.cancer.gov/cancertopics/pdq/treatment/prostate/HealthProfessional/pa ge 1

Nieder, A. M., Porter, M. P., \& Soloway, M. S. (2008). Radiation therapy for prostate cancer increases subsequent risk of bladder and rectal cancer: A population based cohort study. The Journal of Urology, 180(5), 2005-2009. doi:10.1016/j.juro.2008.07.038

O’Brien, R., Hunt, K., \& Hart, G. (2005). 'Its caveman stuff, but that is to a certain extent how guys still operate': Men's accounts of masculinity and help seeking. Social Science \& Medicine, 61(3), 503-516. doi:10.1016/j.socscimed.2004.12.008

Oliffe, J. (2005). Constructions of masculinity following prostatectomy-induced impotence. Social Science \& Medicine, 60, 2249-2259. doi:10.1016/j.socscimed.2004.10.016

Oliffe, J. (2009). Health behaviors, prostate cancer, and masculinities: A life course perspective. Men and Masculinities, 11(3), 346-366. doi:10.1177/1097184X06298777

Oliffe, J. \& Thorne, S. (2007). Men, masculinities, and prostate cancer: Australian and Canadian perspectives of communication with male physicians. Qualitative Health Research, 17, 149-161. doi:10.1177/1049732306297695

Padilla, G. V., \& Grant, M. M. (1985). Quality of life as a cancer nursing outcome variable. Advances in Nursing Science, 8(1), 450-60. 
Palmer, M. H., Fogarty, L. A., Somerfield, M. R., \& Powel, L. L., (2003). Incontinence after prostatectomy: Coping with incontinence after prostate cancer surgery. Oncology Nursing Forum, 30(2), 229-238. doi:10.1188/03.ONF.229-238

Parker, C. (2004). Active surveillance: towards a new paradigm in the management of early prostate cancer. Lancet Oncology, 5, 101-106.

Patel, M. I., DeConcini, D. T., Lopez-Corna, E., Ohori, M., Wheeler, T., \& Scardino, P. T. (2004). An analysis of men with clinically localized prostate cancer. Lancet Oncology, 5, 101-106. doi:10.1097/01.ju.0000118224.54949.78

Patsdaughter, C. A., Kelley, B. R., Babington, L. M., \& Dyer, J. G. (2005). A comparison of sexual risk behaviors of Dominican adolescents in their homeland and in the United States. Journal of Multicultural Nursing and Health, 11(1), 63-68.

Peeters, S. T., Heemsbergen, W. D., Koper, P. C., van Putten, W. L., Slot, A., Dielwart, M. F., . . Lebesque, J. V. (2006). Dose-response in radiotherapy for localized prostate cancer: results of the Dutch multicenter randomized phase III trial comparing 68 Gy of radiotherapy with 78 Gy. Journal of Clinical Oncology, 24(13), 1990-1996. doi:10.1200/JCO.2005.05.2530

Penson, D. F., Feng, A., Kuniyuki, A., McClerran, D., Albertsen, P. C., Deapen, D., . . . Stanford, J. L. (2003). General quality of life 2 years following treatment for prostate cancer: What influences outcomes? Results from the Prostate Cancer Outcomes Study. Journal of Clinical Oncology, 21(6), 1147-1154. doi:10.1200/JCO.2003.07.139

Penson, D. F., \& Litwin, M. S. (2003). Quality of life after treatment for prostate cancer. Current Urology Reports, 4(3), 185-195. doi:10.1007/s11934-003-0068-1

Penson, F. F., McLerran, D., Feng, Z., Li, L., Albertsen, P. C., Gilliland, F. D., . . . Stanford, J. I. (2005). Five-year urinary and sexual outcomes after radical prostatectomy: Results from the prostate cancer outcomes study. The Journal of Urology, 173, 1701-1705. doi:10.1097/01.ju.0000154637.38262.3a

Penson, D. F., Rossignol, M., Sartor, A. O., Scardino, P. T., \& Abenhaim, L. L. (2008). Prostate cancer: Epidemiology and health related quality of life. Urology, 72, S3S11. doi:10.1016/j.urology.2008.10.006.

Peterson, J. L., \& Bakeman, R. (2006). Impact of beliefs about HIV treatment and per condom norms on risky sexual behavior among gay and bisexual men. Journal of Community Psychology, 34, 37-46. doi:10.1002/jcop.20082 
Pleck, J. H., Sonenstein, F., \& Ku, L. C. (1994). Attitudes toward male roles among adolescent males: A discriminant validity analysis. Sex Roles, 30, 481-501. doi:10.1007/BF01420798

Polit, D. F., \& Beck, C. T. (2004). Nursing research: Principles and methods. Philadelphia, PA: Lippincott Williams \& Wilkins.

Pollack, A., Zagars, G. K., Starkschall, G., Antolak, J. A., Lee, J. J., Huang, E., . . . Rosen, I. (2002). Prostate cancer radiation dose response: results of the M. D. Anderson phase III randomized trial. International Journal of Radiation Oncology, Biology, and Physics, 53(5), 1097-1105. doi:10.1016/S03603016(02)02829-8

Potosky, A. L., Davis, W. W., Hoffman, R. M., Stanford, J. L., Stephenson, A. A., Penson, D. F., \& Harlan, L. C. (2004). Five-year outcomes after prostatectomy or radiotherapy for prostate cancer: The Prostate Cancer Outcomes study. Journal of National Cancer Institute, 96(18), 1358-1367. doi:10.1093/jnci/djh259

Potosky, A. L., Legler, J., Albertsen, P. C., Stanford, J. L., Gilliland, F. D., Hamilton, A. S., . . Harlan, L. C. (2000). Health outcomes after prostatectomy or radiotherapy for prostate cancer: Results from the Prostate Cancer Outcomes Study. Journal of the National Cancer Institute, 92(19), 1582-1592. doi:10.1093/jnci/92.19.1582

Potosky, A. L., Reeve, B. B., Clegg, L. X., Hoffman, R. M., Stephenson, R. A., Albertsen, P. C., . . . Stanford, J. L. (2002). Quality of life following localized prostate cancer treated initially with androgen deprivation therapy or no therapy. Journal of the National Cancer Institute, 94(6), 430-437. doi:10.1093/jnci/94.6.430

Prostate Cancer Foundation. (2011). Prostate cancer FAQs. Retrieved from http://www.pcf.org/site/c.leJRIROrEpH/b.5800851/k.645A/Prostate_Cancer_FA Qs.htm

Ragde, H., Blasko, J. C., Grimm, P. D., Kenny, G. M., Sylvester, J. E., Hoak, D. C., . . . Cavanagh, W. (1997). Interstitial iodine-125 radiation without adjuvant therapy in the treatment of clinically localized prostate carcinoma. Cancer, 80(3), 442453. doi:10.1002/(SICI)1097-0142(19970801)80:3<442

Ramsey, S. D. (2008). How should we value lives lost to cancer? Journal of National Cancer Institute, 100 (24), 1742-1743. doi:10.1093/jnci/djn434

Resnick, M. J., Koyama, T., Fan, K. H., Albertsen, P. C., Goodman, M., Hamilton, A. S., Hoffman, R. M., ... Penson, D. F. (2013). Long-term functional outcomes after treatment for localized prostate cancer. New England Journal of Medicine, 368, 5, 436-445. doi:10.1056/NEJMoa1209978 
Rivera-Ramoz, Z. A., \& Buki, L. P. (2011). I will no longer be a man! Manliness and prostate cancer screening among Latino men. Psychology of Men \& Masculinity, 12(1), 13-25. doi:10.1037/a0020624

Robinson, J. W., Saliken, J. C., Donnelly, B. J., Barnes, P., \& Guyn, L. (1999). Qualityof-life outcomes for men treated with cryosurgery for localized prostate carcinoma. Cancer, 86,(9), 1793-1801. doi:10.1002/(SICI)10970142(19991101)86:9<1793

Rondorf-Klym, L. M., \& Colling, J. (2003). Quality of life after radical prostatectomy. Oncology Nurses Foundation, 30(2), E24-E32. doi:10.1188/03.ONF.E24-E32

Sacco, E., Prayer-Galetti, T., Pinto, F., Fracalanza, S., Betto, G., Pagano, F., \& Artibani, W. (2006). Urinary incontinence after radical prostatectomy: Incidence by definition, risk factors and temporal trend in a large series with a long-term follow-up. British Journal of Urology, 97(6), 1234-1241. doi:10.1111/j.1464410X.2006.06185.x

Sanda, M. G., Dunn, R. L., Michalski, J., Sandler, H. M., Northouse, L., Hembroff, L., . . .Wei, J. T. (2008). Quality of life and satisfaction with outcome among prostatecancer survivors. New England Journal of Medicine, 358(12), 1250-1261. doi/full/10.1056/NEJMoa074311

Sanders, S., Pedro, L.W., Bantum, E., \& Galbraith, M. E. (2006). Couples surviving prostate cancer: Long-term intimacy needs and concerns following treatment. Clinical Journal of Oncology Nursing, 10, 503-509. doi:10.1188/06.CJON.503508

Sathya, J. R., Davis, I. R., Julian, J. A., Guo, Q., Daya, D., Dayes, I. S., . . Levine, M. (2005). Randomized trial comparing iridium implant plus external-bean radiation therapy with external-bean radiation therapy alone in node-negative locally advanced cancer of the prostate. Journal of Clinical Oncology, 23, 1192-1199. doi:10.1200/JCO.2005.06.154

Savoie, M., Kim, S. S., \& Soloway ,M. S. (2003). A prospective study measuring penile length in men treated with radical prostatectomy for prostate cancer. The Journal of Urology, 169(4), 1462-1464. doi:10.1097/01.ju.0000053720.93303.33

Schellhammer, P. F., Jordan, G. H., \& el-Mahdi, A. M. (1986). Pelvic complications after interstitial and external beam irradiation of urologic and gynecologic malignancy. World Journal of Surgery, 10(2), 259-268. doi:10.1007/BF01658142 
Schopp, L. H., Good, G. E., Mazurek, M. O., Barker, K. B., \& Stucky, R. C. (2007). Masculine role variables and outcomes among men with spinal cord injury. Disability and Rehabilitation, 29(8), 625-633. doi:10.1080/09638280600902620

Schopp, L. H., Good, G. E., Barker, K. B., Mazurek, M. O., \& Hathaway, S. L. (2006). Masculine role adherence and outcomes among men with traumatic brain injury. Brain Injury, 20(11), 1155-1162. doi:10.1080/02699050600983735

Schuessler, W. W, Vancaillie, T. G, Reich, H., \& Griffith, D. P. (1991). Transperitoneal endosurgical lymphadenectomy in patients with localized prostate cancer. The Journal of Urology, 145(5), 988-991.

Sheldon, T. A., \& Parker, H. (1992). Race and ethnicity in health research. Journal of Public Health, 14(2), 104-110.

Shahinian, V. B., Kuo, Y. F., Freeman, J. L., \& Doodwin, J. S. (2005). Risk of fracture after androgen deprivation for prostate cancer. New England Journal of Medicine, 352(2), 154-164. doi:10.1056/NEJMoa041943

Shelley, M., Wilt, T. J., Coles, B., \& Mason, M. D. (2007). Cryotherapy for localized prostate cancer. Cochrane Database Systematic Reviews, 3, CD005010. doi:10.1002/14651858.CD005010.pub2

Shikanov, S. A., Eng, M. K., Bernstein, A. J., Katz, M., Zagaja, G. P., Shalhav, A. L., \& Zorn, K. C. (2008). Urinary and sexual quality of life 1 year following robotic assisted laparoscopic radical prostatectomy. The Journal of Urology, 180(2), 663-667. doi:10.1016/j.juro.2008.04.013

Siegel, T., Moul, J. W., Spevak, M., Alvord, W. G., \& Costabile, R. A. (2001). The development of erectile dysfunction in men treated for prostate cancer. The Journal of Urology, 165, 430-435. doi:10.1097/00005392-200102000-00019

Siegel, R., Naishadham, D., \& Jemal, A. (2013). Cancer statistics, 2013. CA: A Cancer Journal for Clinicians, 63, 1, 11-30. doi:10.3322/cac.21166

Snyder, C. F., Frick, K. D., Blackford, A. L., Herbert, R. J., Neville, B. A., Carducci, M. A. \& Earle, C. C. (2010). How does initial treatment choice affect shortterm and long-term costs for clinically localized prostate cancer? Cancer, 116, 5391-5399. doi:10.1002.cncr.25517

Solomon, S., Greenberg, J., \& Pyszczynski, T. (1991). A terror management theory of social behavior: The psychological functions of self esteem and cultural worldviews. Advances in Experimental Social Psychology, 24, 93-159. 
Soloway, M. S., Schellhammer, P. F., Smith, J. A., Chodak, G. W., \& Kennealey, G. T. (1996). Bicalutamide in the treatment of advanced prostatic carcinoma: A phase II multicenter trial. Urology , 47, 33-37. doi:10.1016/S0090-4295(96)80006-9

Spanier, G. B. (1976). Measuring dyadic adjustment: New scales for assessing the quality of marriage and similar dyads. Journal of Marriage and Family, 38, 1528.

Stanford, J. L., Feng, Z., Hamilton, A.S., Gilliland, F. D., Stephenson, R. A., Eley, J. W., .. \& \& Potosky, A. L. (2000). Urinary and sexual function after radical prostatectomy for clinically localized prostate cancer. Journal of the American Medical Association, 283(3), 354-360. doi:10.1001/jama.283.3.354

Stansburry, J. P., Mathew-Chapman, M., \& Grant, K. E. (2003). Gender schema and prostate cancer: Veterans' cultural model of masculinity. Medical Anthropology, 22, 175-204. doi:10.1080/01459740306765

Steineck, G., Helgesen, F., Adolfsson, J., Dickman, P. W., Johansson, J. E., Norlen, B. J., ... the Scandinavian Prostate Cancer Group Study Number 4. (2002). Quality of life after radical prostatectomy or watchful waiting. The New England Journal of Medicine, 347, 790-796. doi:10.1056/NEJMoa021483

Stewart, A. L., \& Ware, J. E. (1992). Measuring functioning and well-being: The Medical Outcomes Study Approach. Durham, NC: Duke University Press.

Stibbe, A. (2004). Health and the social construction of masculinity in Men's Health magazine. Men and Masculinities, 7, 31-51. doi:10.1177/1097184X03257441

Sun, M., Lughezzani, G., Alasker, A., Isbarna, H., Jeldresa, C., Shariata, S. F., . . .Karakiewicza, P. I. (2010). Comparative study of inguinal hernia repair after radical prostatectomy, prostate biopsy, transurethral resection of the prostate or pelvic lymph node dissection. The Journal of Urology, 183(3), 970-975. doi:10.1016/j.juro.2009.11.036

Talcott, J. A., Rossi, C., Shipley, W. U., Clark, J. A., Slater, J. D., Niemierko, A., \& Zietman, A. L. (2010). Patient-reported long-term outcomes after conventional and high-dose combined proton and photon radiation for early prostate cancer. Journal of the American Medical Association, 303(11), 1046-1053. doi:10.1001/jama.2010.287

Tsai, H. K., D’Amico, A. V., Sadetsky, N., Chen, M. H., \& Carroll, P. R. (2007). Androgen deprivation therapy for localized prostate cancer and the risk of cardiovascular mortality. Journal of National Cancer Institute, 99(20), 15161524. doi:10.1093/jnci/dmj168 
U.S. Department of Health and Human Services Public Health Services National Institutes of Health. (1990). Quality of life assessment in cancer clinical trials. Report of the workshop on quality of life research in cancer clinical trials. Bethesda, Maryland.

U.S. Department of Health and Human Services. (2009). Research involving individuals with questionable capacity to consent: Points to consider. Retrieved from http://grants.nih.gov/grants/policy/questionablecapacity.htm

USMD Prostate Cancer Center. (n.d.). Will my insurance company cover prostate cancer screening and treatment? Retrieved from http://www.usmdpcc.com/faq.html\#insurance

United Healthcare. (2011). United Healthcare Medicare Advantage Plans: Urinary incontinence. Retrieved from https://www.unitedhealthcareonline.com/ccmcontent/ProviderII/UHC/enUS/Assets/ProviderStaticFiles/ProviderStaticFilesPdf/Tools\%20and\%20Resource s/Policies\%20and\%20Protocols/UnitedHealthcare\%20Medicare\%20Coverage/Im potence_Treatment_UHCMA_CS.pdf

United Healthcare. (2012). Nerve graft to restore erectile function during radical prostatectomy. Retrieved from https://www.unitedhealthcareonline.com/ccmcontent/ProviderII/UHC/enUS/Assets/ProviderStaticFiles/ProviderStaticFilesPdf/Tools\%20and\%20Resource s/Policies\%20and\%20Protocols/Medical\%20Policies/Medical\%20Policies/Nerve _Graft_Res_Erect_Funct_Rad_Prost.pdf

U.S. Census Bureau. (2010). State \& county quickfacts. Retrieved from http://quickfacts.census.gov/qfd/states/12/124

Victorian, B. (2008). Urologists urge coverage of ED treatment following prostatectomy. Oncology Times, 30(14), 16-17. doi:10.1097/01.COT.0000333623.72530.4f

Waaler, G., \& Stenwig, A. E. (1993). Prognosis of localized prostatic cancer managed by "watch and wait" policy. British Journal of Urology, 72(2), 214-9, 1993.

Wall, D., \& Kristjanson, L. (2005). Men, culture, and hegemonic masculinity: Understanding the experience of prostate cancer. Nursing Inquiry, 12(2), 87-97. doi:10.1111/j.14401800.2005.00258.x

Wallace, M. (2003). Uncertainty and quality of life of older men who undergo watchful waiting for prostate cancer. Oncology Nursing Forum, 30(2), 303-309. doi:10.1188/03.ONF.303-309 
Ward, E., Jemal, A., Cokkinides, V., Singh, G. K., Cardinez, C., Ghafoor, A., \& Thun, M. (2004). Cancer disparities by race/ethnicity and socioeconomic status. $C A: A$ Cancer Journal for Clinicians, 54, 78-93. doi:10.3322/canjclin.54.2.78

Ware, J. J. (1995). The status of health assessment. Annual Review of Public Health, 16, 327-254.

Ware, J. J., Kosinski, M., \& Dewey, J. (2000). How to score Version 2 of the SF-36® Health Survey. Lincoln, RI: QualityMetric.

Ware, J. J., Kosinski, M., \& Gandek, B. (2000). SF-36® Health Survey: Manual \& Interpretation Guide. Lincoln, RI: QualityMetric.

Ware, J. J., Kosinski, M., \& Keller, S. K. (1994). SF-36 Physical and Mental Health Summary Scales: A user's manual. Boston, MA: Health Institute, New England Medical Center.

Ware, J. E., Snow, K. K., Kosinski, M., \& Gandek, B. (1993). SF-36 Health Survey Manual and Interpretation Guide. Boston, MA: Health Institute, New England Medical Center.

Weber, B. A., Roberts, B. L., Chumbler, N. R., Mills, T. L., \& Algood, C. (2007). Urinary, sexual, and bowel dysfunction and bother after radical prostatectomy. Urologic Nursing, 27, 6, 527-533.

Wei, J. T., Dunn, R. L., Sandler, H. M., McLaughlin, P. W., Montie, J. E., Litwin, M. S., ... Sanda, G. (2002). Comprehensive comparison of heath related quality of life after contemporary therapies for localized prostate cancer. Journal of Oncology Nursing, 20, 557-566. doi:10.1200/JCO.20.2.557

Weiss, J. W., \& Garbanati, J. A. (2006). Effects of acculturation and social norms on adolescent smoking among Asian-American subgroups. Journal of Ethnicity in Substance Abuse, 5, 75-90. doi:10.1300/J233v05n02_05

Whitmore, W. F. Jr. (1994). Expectant management of clinically localized prostatic cancer. Seminars in Oncology, 21(5), 560-568.

Wilson, I. B., \& Cleary, P. D. (1995). Linking clinical variables with health-related quality of life: A conceptual model of patient outcomes. Journal of the American Medical Association, 273(1), 59-65. doi:10.1001/jama.1995.03520250075037

Wilson, L. S., Tesoro, R., Elkin, E. P., Sadetsky, N., Broering, J. M., Latini, D. M., DuChane, J., Modt, R. R., \& Carroll, P. R. (2007). Cumulative cost pattern comparison of prostate cancer treatments. Cancer, 109(3), 518-527. 
Wilt, T. J., MacDonald, R., Rutks, I., Shamliyan, T. A., Taylor, B. C., \& Kane, R. L. (2008). Systemic review: Comparative effectiveness and harms of treatments for clinically localized prostate cancer. Annals of Internal Medicine, 148(6), 435448. doi:10.7326/0003-4819-148-6-200803180-00209

Witjes, W. P., Schulman, C. C., \& Debruyne, F. M. (1997). Preliminary results of a prospective randomized study comparing radical prostatectomy versus radical prostatectomy associated with neoadjuvant hormonal combination therapy in T2-3 N0 M0 prostatic carcinoma. Urology, 49, 65-69. doi:10.1016/S00904295(97)00171-4

Wong, Y. N., Mitra, N., Hudes, G., Localio, R., Schwarts, S., Wan, F., . . Armstrong, K. (2006). Survival associated with treatment vs observation of localized prostate cancer in elderly. Journal of the American Medical Association, 296(22), 26832693. doi:10.1001/jama.296.22.2683

Woods, D., Montgomery, S. B., \& Herring, R. P. (2004). Recruiting Black/African American men for research on prostate cancer prevention. Cancer, 100(5), 10171025. doi:10.1002/cncr.20029

Wootten, A. C., Burney, S., Foroud, F., Frydenberg, M., Coleman, G., \& Ng, K. T. (2007). Psychological adjustment of survivors of localized prostate cancer: Investigating the role of adjustment, cognitive appraisal, and coping style. PsychoOncology, 16, 994-1002. doi:10.1002/pon.1159

World Health Organization. (1947). Chronicles of World Health Organization, 1, 13.

World Health Organization Division of Mental Health. (1993). WHO-QOL study protocol: The development of the World Health Organization quality of life assessment instrument. Geneva, Switzerland:

Wu, L. M., Mohamed, N. E., Winkel, G., \& Diefenbach, M. A. (2013). Patient and spouse illness beliefs and quality of life in prostate cancer patients. Psychology and Health, 28 (4), 355-368. doi:10.1080/08870446.2012.722219

Wysowski, D. K., Freiman, J. P., Tourtelot, J. B., \& Horton, M. L. (1993). Fatal and nonfatal hepatotoxicity associated with flutamide. Annals of Internal Medicine, $118(11), 860-864$.

Xiao, H., Warrick, C., \& Huang, Y. (2009). Prostate cancer treatment patterns among racial/ ethnic groups in Florida. Journal of the National Medical Association, 101(9), 936-943. 
Yancy, A. K., Ortega, A. N., \& Kumanyika, S. K. (2006). Effective recruitment and retention of minority research participants. Annual Review of Public Health, 27, 1-28. doi:10.1146/annurev.publhealth.27.021405

Yao, S. L., \& Lu-Yao, G. (1999). Population-based study of relationships between hospital volume of prostatectomies, patient outcomes, and length of hospital stay. Journal of the National Cancer Institute, 91(22), 1950-1956. doi:10.1093/jnci/91.22.1950

Yobroff, K. R., Lawrence, W. F., Clausser, S., Davis, W. W., \& Brown, M. L. (2004). Burden of illness in cancer survivors: Findings from a population-based national sample. Journal of National Cancer Institute, 96(17), 1322-1330. doi:10.1093/jnci/djh255

Youngblut, J. M., Brooten, D., \& Menzies, V. (2006). Psychometric properties of the Spanish versions of the FACES II and Dyadic Adjustment Scale. Journal of Nursing Measurement, 14(3), 181-189.

Zelefsky, M. J., Fuks, Z., Hunt, M., Yamada, Y., Marion, C., Ling, C. C., . . . Leibel, S. A. (2002). High-dose intensity radiation therapy for prostate cancer: Early toxicity and biochemical outcome in 772 patients. International Journal of Radiation Oncology, 53(5), 1111-1116. doi:10.1016/S0360-3016(02)02857-2

Zhan, L. (1992). Quality of life: Conceptual and measurement issues. Journal of Advanced Nursing, 17, 795-800. doi:10.1111/j.1365-2648.1992.tb02000.x

Zietman, A. L., DeSilvio, M. L., Slater, J. D., Carl J., Rossi, C. J., Miller, D. W., . . Shipley, W. U. (2005). Comparison of conventional-dose vs high-dose conformal radiation therapy in clinically localized adenocarcinoma of the prostate: A randomized controlled trial. Journal of American Medical Association, 294(10), 1233-1239. doi:10.1001/jama.294.10.1233 
VITA

\section{SUHA BALLOUT}

DATE

2013

2007

2000
EDUCATION

Florida International University

Miami, Florida 33199

American University of

Beirut

Beirut, Lebanon

American University of

Beirut

Beirut, Lebanon
DEGREE

Doctor of Philosophy in

Nursing Science (PhD)

Masters of Science in Nursing (MSN)

Bachelor of Science in Nursing (BSN)
MAJOR

Nursing

Adult Care

Nursing

\section{PUBLICATIONS AND PRESENTATIONS}

Journal Articles (Refereed)

Kulwicki, A., Aswad, B., Ballout, S. (2010). Barriers in the Utilization of Domestic Violence Services Among Arab Immigrant Women: Perceptions of Professionals, Service Providers \& Community Leaders. Journal of Family Violence, 25(8), 727-735.

Ballout, S., Noureddine, S., Hujeir, H., \& Kanazi, G. (2010). Psychometric evaluation of Arabic brief pain inventory; A pilot study on a sample of Lebanese cancer patients. Journal of Pain and Symptom Management.

\section{Book Chapters}

Kulwicki, A., \& Ballout, S. (2011). People of Arab Heritage. In L. Purnell \& B. Paulanka (3nd Eds.), Transcultural Health Care. Philadelphia, PA: F.A Davis Company. Accepted for publication.

Ballout S. (2004). Pain Assessment. In J. Weber \& J. Kelley ( $3^{\text {rd }}$ Ed.) Health Assessment in Nursing. Philadelphia: Lippincott Williams \& Wilkins.

Master's Thesis

Ballout, S., Noureddine, S., Hujeir, H., \& Kanazi, G. (2007). Psychometric Evaluation of Arabic Brief Pain Inventory; A Pilot Study on a sample of Lebanese cancer patients. 


\section{Dissertation (In Progress)}

Ballout, S., Kulwicki, A., Patsdaughter, C. A., Brown, E., Lavendar, A. (2012). Quality of Life in Men with Prostate Cancer.

Journal Articles (In Review)

Kulwicki, A., Ballout, S., \& Farraj, M. (2012). PTSD in Arab American Women. Journal of Transcultural Nursing. (In Press).

Kulwicki, A., Kilgore, C., Ballout, S., \& Hammad, A., Dervartanian, H., \& Hymes, R. (2012). Intimate partner violence, depression, and barriers to service in Arab American women.

Presentations

\section{International Presentations}

Ballout, S. (2007). Psychometric Evaluation of Arabic Brief Pain Inventory; A Pilot Study on a sample of Lebanese Cancer Patients. $8^{\text {th }}$ Annual Interdisciplinary Research Conference, School of Nursing and Midwifery, Trinity College Dublin, Ireland

National Presentations (United States)

Kulwicki, A., \& Ballout S. (2012). Acculturation and Mental Health Outcomes of Arab American Women. Southern Nursing Research Conference, New Orleans.

Ballout, S. \& Kulwicki, A. (2011). Predictors of Acculturation in Arab American Women. Southern Nursing Research Society Conference. Jacksonville,FL.

Ballout, S. (2011). Coping and Adjustment of Men with Prostate Cancer. 12th National Conference on Cancer Nursing Research, Oncology Nurses Society, Los Angeles, CA.

Ballout, S. (2010). Coping and Adjustment of Men with Prostate Cancer. 4th Annual South Florida Research Consortium: Best Evidence from Research: The Gateway to Excellent Care. Jungle Island, Miami, Fl.

Kulwicki, A., Ballout, S., Farrag, M., Hymes, R. (2010). Posttraumatic Stress Disorders in Arab American Women. The 42nd Asia-Pacific Academic Consortium for Public Health (APACPH) Conference, Nusa Dua, Bali, Indonesia.

Kulwicki, A., \& Ballout, S. (2010). Barriers in the utilization of domestic violence Services among Arab American immigrant women: Perceptions of professionals, service providers, and community leaders" 4th Annual South Florida Research Consortium: Best Evidence from Research: The Gateway to Excellent Care. Jungle Island, Miami, Fl.

Ballout, S. (2009). Psychometric Evaluation of Arabic Brief Pain Inventory; A Pilot Study on a sample of Lebanese Cancer Patients. 10th National Conference on Cancer Nursing Research., Oncology Nurses Society, Orlando, Florida. 